\title{
Dietary Supplement Laboratory Quality Assurance Program: Exercise J Final Report
}

\author{
Melissa M. Phillips \\ Catherine A. Rimmer \\ Laura J. Wood
}

Mary Bedner

Kaitlyn D. Chieh

Rick L. Paul 
NISTIR 7997

\section{Dietary Supplement Laboratory Quality Assurance Program: Exercise J Final Report}

Melissa M. Phillips Catherine A. Rimmer

Laura J. Wood

Mary Bedner

Kaitlyn D. Chieh

Rick L. Paul

Chemical Sciences Division

Material Measurement Laboratory

http://dx.doi.org/10.6028/NIST.IR.7997

April 2014

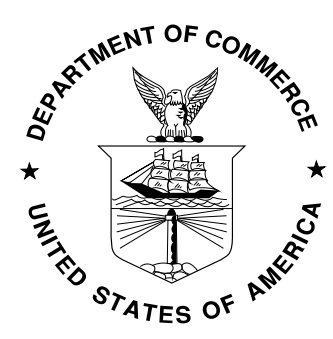

U.S. Department of Commerce

Penny Pritzker, Secretary

National Institute of Standards and Technology

Patrick D. Gallagher, Director 


\section{TABLE OF CONTENTS}

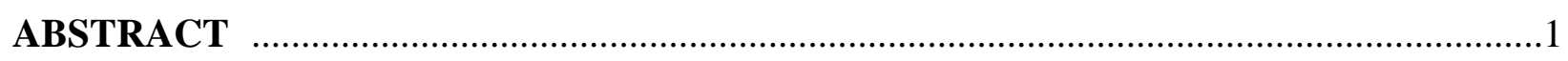

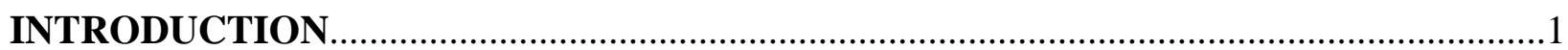

\section{OVERVIEW OF DATA TREATMENT AND REPRESENTATION}

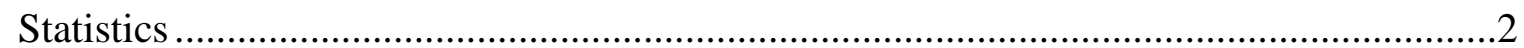

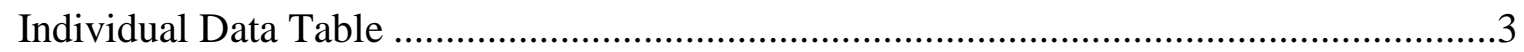

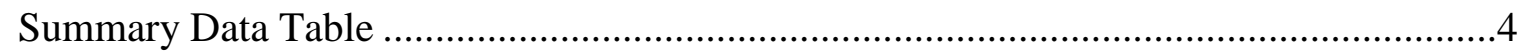

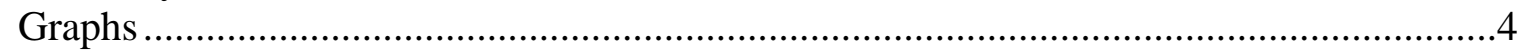

Data Summary View (Method Comparison Data Summary View) ………………...4

Sample/Control Comparison View (Sample/Sample Comparison View)................5

Composition View …………………………………………………………......

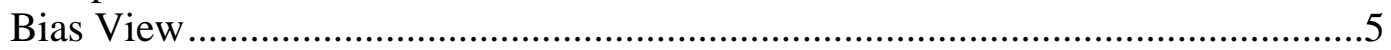

NUTRITIONAL ELEMENTS (Ca, Mg, Zn) IN NATURAL AND ENHANCED WATERS 6

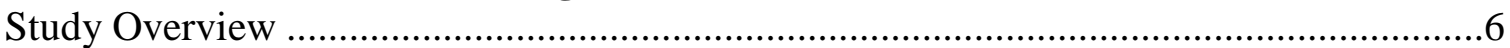

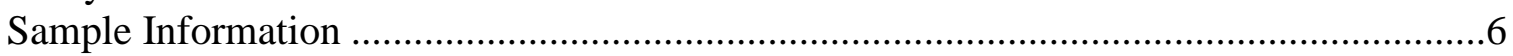

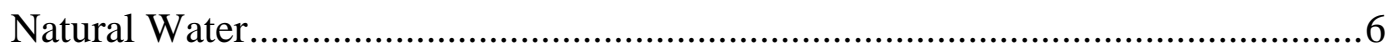

Enhanced Water ........................................................................................

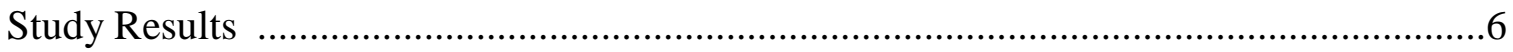

Technical Recommendations ....................................................................................

Table 1. Individual data summary table (NIST) for nutritional elements in water .............8

Table 2. Data summary table for calcium in natural and enhanced waters.........................9

Table 3. Data summary table for magnesium in natural and enhanced waters.................10

Table 4. Data summary table for zinc in natural and enhanced waters ............................11

Figure 2. Calcium in enhanced water .............................................................................13

Figure 3. Magnesium in SRM 1643e Trace Elements in Water .....................................14

Figure 4. Magnesium in enhanced water ................................................................15

Figure 5. Zinc in SRM 1643e Trace Elements in Water ..............................................16

Figure 6. Zinc in enhanced water ..................................................................17

Figure 7. Calcium in SRM 1643e Trace Elements in Water ..........................................18

Figure 8. Calcium in enhanced water ..........................................................................19

Figure 9. Magnesium in SRM 1643e Trace Elements in Water .....................................20

Figure 10. Magnesium in enhanced water ...................................................................21

Figure 11. Zinc in SRM 1643e Trace Elements in Water ..............................................22

Figure 12. Zinc in enhanced water .............................................................................23

Figure 13. Calcium in SRM 1643e Trace Elements in Water and enhanced water ........24

Figure 14. Magnesium in SRM 1643e Trace Elements in Water and enhanced water ...25

Figure 15. Zinc in SRM 1643e Trace Elements in Water and enhanced water ...............26

TOXIC ELEMENTS (As) IN ST. JOHN'S WORT DIETARY SUPPLEMENTS …...........27

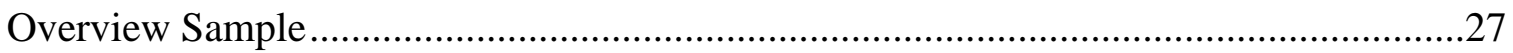

St. John's Wort Aerial Parts.................................................................................22

St. John's Wort Methanol Extract.......................................................................27

Study Results 27

Technical Recommendations 
Table 5. Individual data summary table (NIST) for arsenic in St. John’s Wort dietary

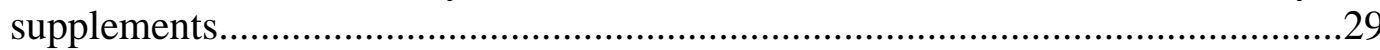

Table 6. Data summary table for arsenic in St. John's Wort dietary supplements ..........30

Figure 16. Arsenic in candidate SRM 3264 Hypericum perforatum L. (St. John's Wort) Methanol Extract (data summary view - digestion method) ................................31

Figure 17. Arsenic in candidate SRM 3262 Hypericum perforatum L. (St. John's Wort) Aerial Parts (data summary view - digestion method)

Figure 18. Arsenic in candidate SRM 3262 Hypericum perforatum L. (St. John's Wort) Aerial Parts and candidate SRM 3264 Hypericum perforatum L. (St. John’s Wort) Methanol Extract (sample/sample comparison view)

WATER-SOLUBLE VITAMINS $\left(B_{5}, B_{6}\right)$ IN DIETARY SUPPLEMENTS........................34

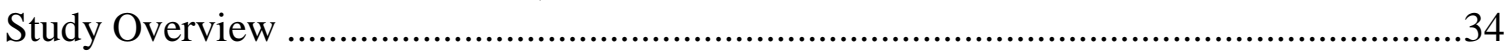

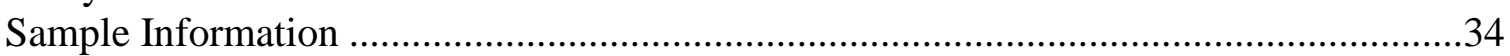

Multivitamin/Multielement Tablets .....................................................................34

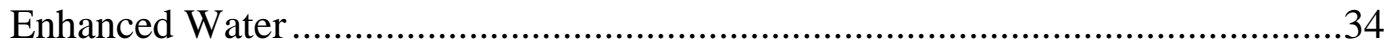

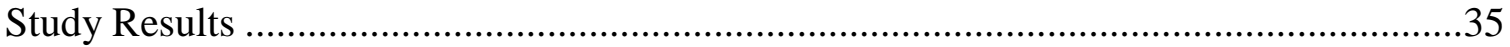

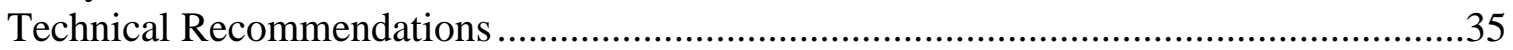

Table 7. Individual data summary table (NIST) for water-soluble vitamins in dietary

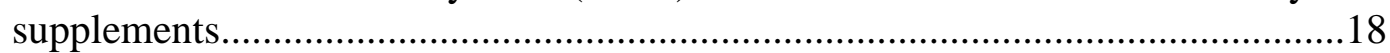

Table 8. Data summary table for vitamin $\mathrm{B}_{5}$ (pantothenic acid) in dietary supplements 19

Table 9. Data summary table for vitamin $\mathrm{B}_{6}$ (pyridoxine hydrochloride) in dietary

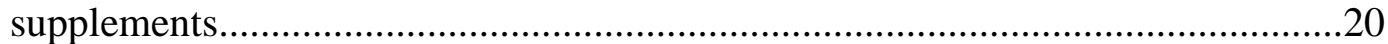

Figure 19. Pantothenic acid in SRM 3280 Multivitamin/Multielement Tablets .............39

Figure 20. Pantothenic acid in enhanced water.......................................................40

Figure 21. Pyridoxine hydrochloride in SRM 3280 Multivitamin/Multielement Tablets 41

Figure 22. Pyridoxine hydrochloride in enhanced water .........................................42

Figure 23. Pantothenic acid in SRM 3280 Multivitamin/Multielement Tablets and

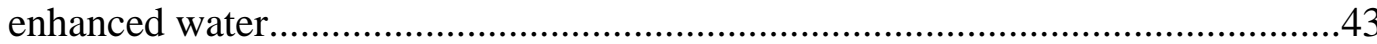

Figure 24. Pyridoxine hydrochloride in SRM 3280 Multivitamin/Multielement Tablets and enhanced water ................................................................................4

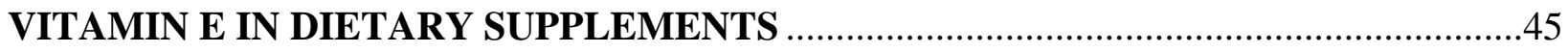

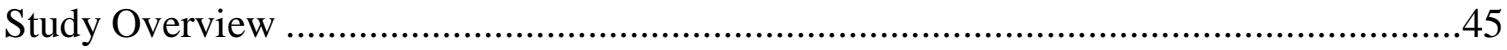

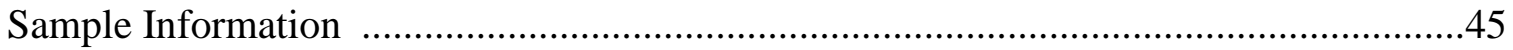

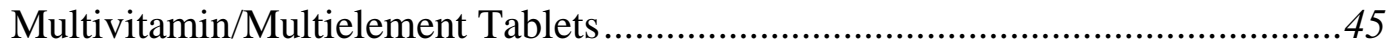

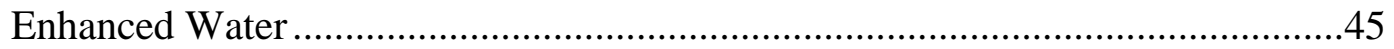

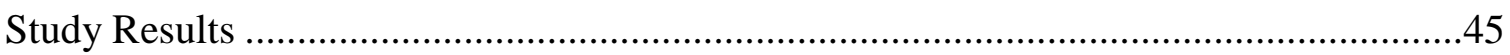

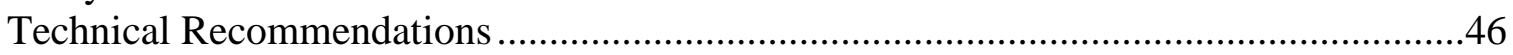

Table 10. Individual data summary table (NIST) for $\alpha$-tocopherol acetate in dietary

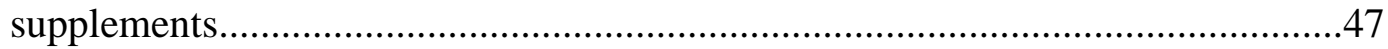

Table 11. Data summary table for $\alpha$-tocopherol acetate in dietary supplements ............48

Figure 25. $\alpha$-Tocopherol acetate in SRM 3280 Multivitamin/Multielement Tablets (data summary view)......................................................................................49

Figure 26. $\alpha$-Tocopherol acetate in enhanced water (data summary view) .....................50

Figure 27. $\alpha$-Tocopherol acetate in SRM 3280 Multivitamin/Multielement Tablets and enhanced water (sample/control comparison view)............................................51 
FATTY ACIDS IN BOTANICAL AND FISH OIL DIETARY SUPPLEMENTS...............52

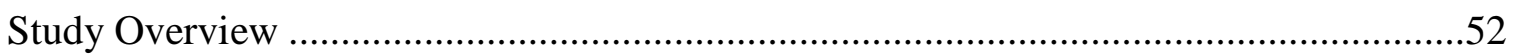

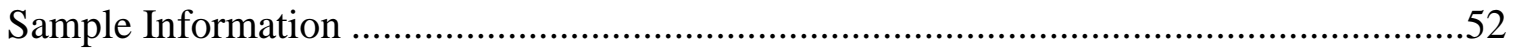

Evening Primrose Oil52

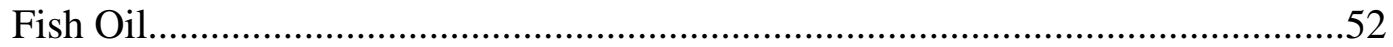

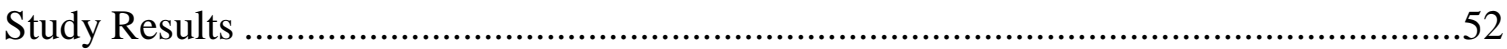

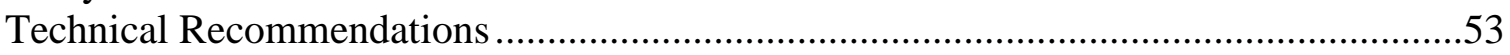

Table 12. Individual data summary table (NIST) for fatty acids in botanical and fish oil

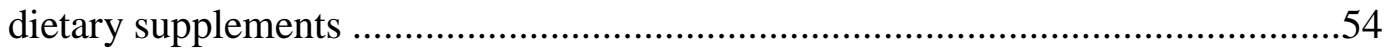

Table 13. Data summary table for linoleic acid in botanical and fish oil dietary

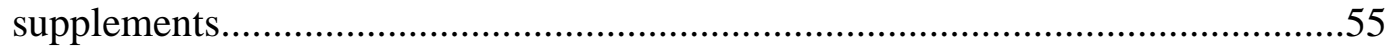

Table 14. Data summary table for $\alpha$-linolenic acid in botanical and fish oil dietary

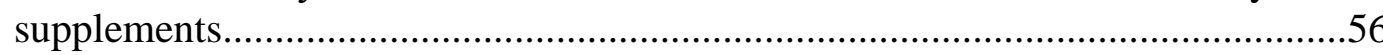

Table 15. Data summary table for $\gamma$-linolenic acid in botanical and fish oil dietary

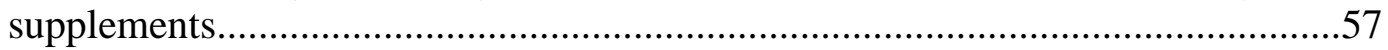

Table 16. Data summary table for arachidonic acid in botanical and fish oil dietary

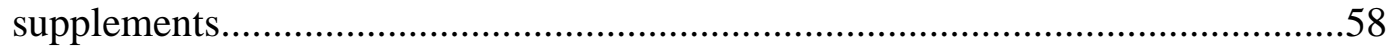

Table 17. Data summary table for EPA in botanical and fish oil dietary supplements ...59

Table 18. Data summary table for DHA in botanical and fish oil dietary supplements ..60

Figure 28. Linoleic acid in SRM 3274-2 Evening Primrose Oil (data summary view) ..61

Figure 29. Linoleic acid in SRM 3275-2 Fish Oil (data summary view) .......................62

Figure 30. $\alpha$-Linolenic acid in SRM 3274-2 Evening Primrose Oil (data summary view)63

Figure 31. $\alpha$-Linolenic acid in SRM 3275-2 Fish Oil (data summary view) ..................64

Figure 32. $\gamma$-Linolenic acid in SRM 3274-2 Evening Primrose Oil (data summary view)65

Figure 33. $\gamma$-Linolenic acid in SRM 3275-2 Fish Oil (data summary view) ..................66

Figure 34. Arachidonic acid in SRM 3275-2 Fish Oil (data summary view).................67

Figure 35. EPA in SRM 3275-2 Fish Oil (data summary view) ...................................68

Figure 36. DHA in SRM 3275-2 Fish Oil (data summary view).................................69

Figure 37. Linoleic acid in SRM 3274-2 Evening Primrose Oil and SRM 3275-2 Fish

Oil-2 (sample/control comparison view) .......................................................70

Figure 38. $\alpha$-Linolenic acid in SRM 3274-2 Evening Primrose Oil and SRM 3275-2 Fish Oil-2 (sample/control comparison view) ........................................................71

Figure 39. $\gamma$-Linolenic acid in SRM 3274-2 Evening Primrose Oil and SRM 3275-2 Fish Oil-2 (sample/control comparison view) ........................................................ 72

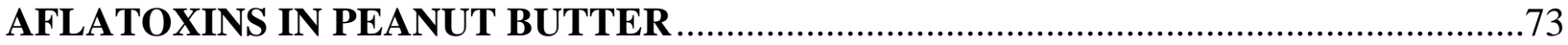

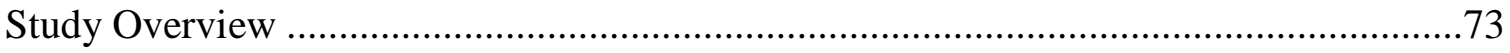

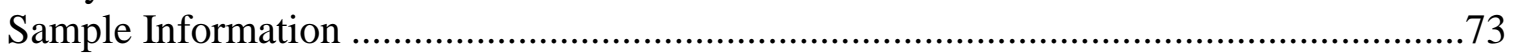

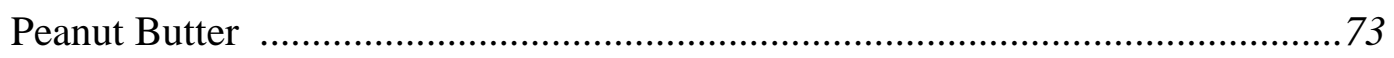

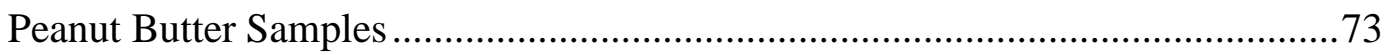

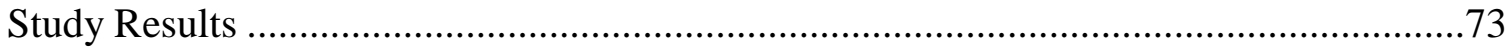

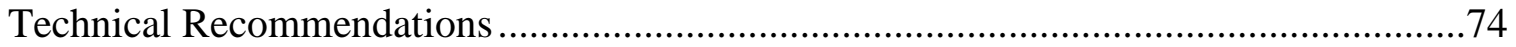

Table 19. Individual data summary table (NIST) for aflatoxins in peanut butter ............75

Table 20. Data summary table for aflatoxin B1 in peanut butter...................................76

Table 21. Data summary table for aflatoxin B2 in peanut butter..................................76

Table 22. Data summary table for aflatoxin G1 in peanut butter..................................77

Table 23. Data summary table for aflatoxin G2 in peanut butter...................................77

Table 24. Data summary table for total aflatoxins in peanut butter ...............................78 
Figure 40. Aflatoxin B1 in SRM 2387 Peanut Butter (data summary view) ...................79

Figure 41. Aflatoxin B2 in SRM 2387 Peanut Butter (data summary view)..................80

Figure 42. Total aflatoxins in SRM 2387 Peanut Butter (data summary view)..............81

Figure 43. Total aflatoxins in peanut butter sample 1 (low) (composition view)............82

Figure 44. Total aflatoxins in peanut butter sample 2 (medium) (composition view).....83

Figure 45. Total aflatoxins in peanut butter sample 3 (high) (composition view)..........84

Figure 46. Aflatoxins in peanut butter sample 1 (low) (bias view) ...............................85

Figure 47. Aflatoxins in peanut butter sample 3 (medium) (bias view) .........................86

Figure 48. Aflatoxins in peanut butter sample 3 (high) (bias view) ..............................87

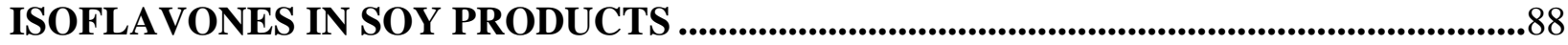

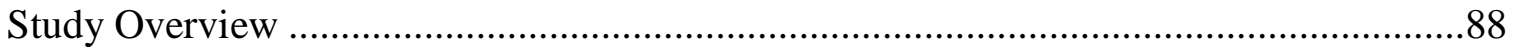

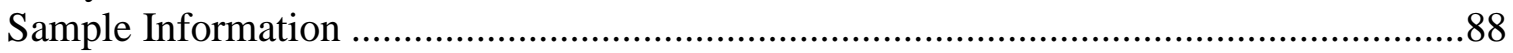

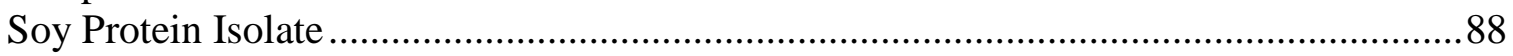

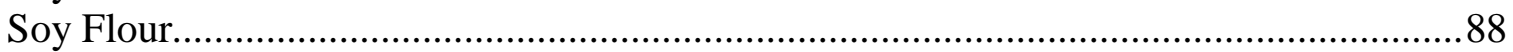

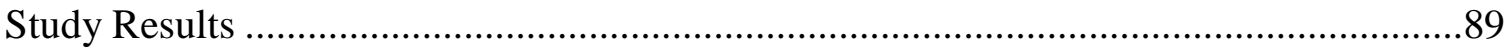

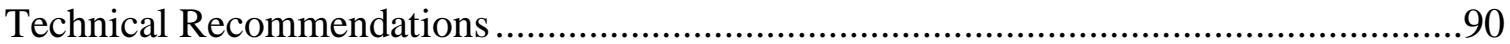

Table 25. Individual data summary table (NIST) for isoflavones in soy dietary

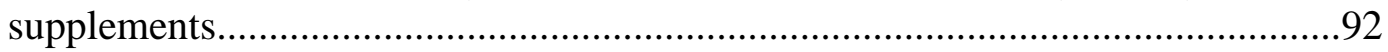

Table 26. Data summary table for daidzin in soy dietary supplements ........................93

Table 27. Data summary table for glycitin in soy dietary supplements ..........................94

Table 28. Data summary table for genistin in soy dietary supplements ........................95

Table 29. Data summary table for daidzein in soy dietary supplements........................96

Table 30. Data summary table for glycitein in soy dietary supplements ........................97

Table 31. Data summary table for genistein in soy dietary supplements.......................98

Table 32. Data summary table for total isoflavones in soy dietary supplements.............99

Figure 49. Daidzin in SRM 3236 Soy Protein Isolate (data summary view, sample preparation comparison) ...........................................................................100

Figure 50. Daidzin in SRM 3234 Soy Flour (data summary view, sample preparation

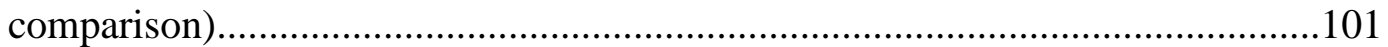

Figure 51. Glycitin in SRM 3236 Soy Protein Isolate (data summary view, sample preparation comparison) ................................................................................102

Figure 52. Glycitin in SRM 3234 Soy Flour (data summary view, sample preparation

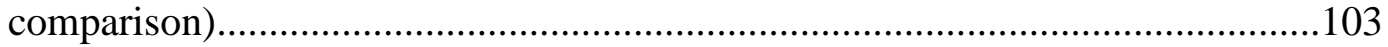

Figure 53. Genistin in SRM 3236 Soy Protein Isolate (data summary view, sample preparation comparison) ............................................................................104

Figure 54. Genistin in SRM 3234 Soy Flour (data summary view, sample preparation

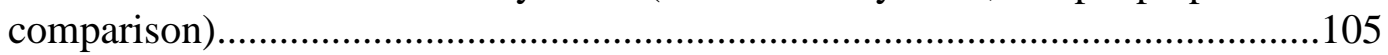

Figure 55. Daidzein in SRM 3236 Soy Protein Isolate (data summary view, sample preparation comparison) …...........................................................................106

Figure 56. Daidzein in SRM 3234 Soy Flour (data summary view, sample preparation

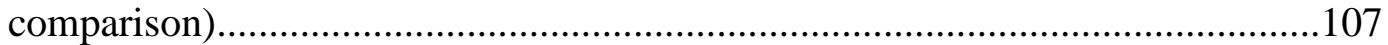

Figure 57. Glycitein in SRM 3236 Soy Protein Isolate (data summary view, sample

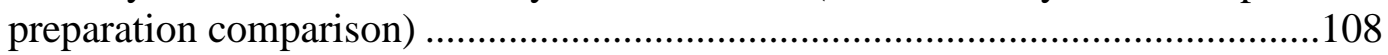

Figure 58. Glycitein in SRM 3234 Soy Flour (data summary view, sample preparation

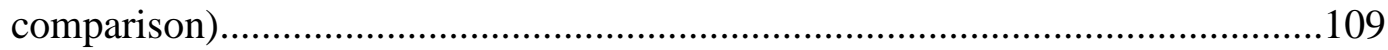

Figure 59. Genistein in SRM 3236 Soy Protein Isolate (data summary view, sample preparation comparison) 
Figure 60. Genistein in SRM 3234 Soy Flour (data summary view, sample preparation comparison).

Figure 61. Total isoflavones in SRM 3236 Soy Protein Isolate (data summary view, sample preparation comparison)

Figure 62. Total isoflavones in SRM 3234 Soy Flour (data summary view, sample preparation comparison)

Figure 63. Daidzin in SRM 3236 Soy Protein Isolate and SRM 3234 Soy Flour (sample/control comparison view).

Figure 64. Glycitin in SRM 3236 Soy Protein Isolate and SRM 3234 Soy Flour (sample/control comparison view).

Figure 65. Genistin in SRM 3236 Soy Protein Isolate and SRM 3234 Soy Flour (sample/control comparison view).

Figure 66. Daidzein in SRM 3236 Soy Protein Isolate and SRM 3234 Soy Flour (sample/control comparison view).

Figure 67. Glycitein in SRM 3236 Soy Protein Isolate and SRM 3234 Soy Flour (sample/control comparison view).

Figure 68. Genistein in SRM 3236 Soy Protein Isolate and SRM 3234 Soy Flour (sample/control comparison view).

Figure 69. Total isoflavones in SRM 3236 Soy Protein Isolate and SRM 3234 Soy Flour (sample/control comparison view).

BOTANICAL IDENTITY OF PURE AND ADULTERATED GINKGO BILOBA

Study Overview

Sample Information

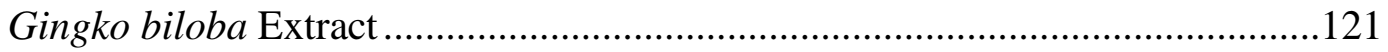

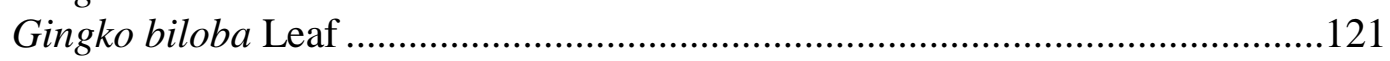

Study Results

Technical Recommendations

Table 33. Individual data summary table (NIST) for botanical identity of pure and adulterated Ginkgo biloba...

Table 34. Data summary table for Ginkgo biloba extracts (yes/no) .............................124

Table 35. Data summary table for Ginkgo biloba extracts (mass percentage). ............125

Table 36. Data summary table for Ginkgo biloba leaves (yes/no)................................126

Table 37. Data summary table for Ginkgo biloba leaves (mass percentage)..................127

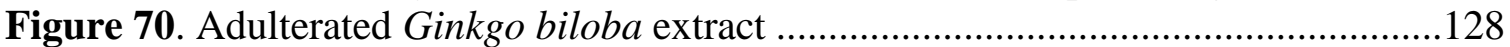

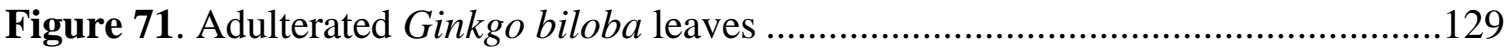




\begin{abstract}
The NIST Dietary Supplement Laboratory Quality Assurance Program (DSQAP) was established in collaboration with the National Institutes of Health (NIH) Office of Dietary Supplements (ODS) in 2007 to enable members of the dietary supplements community to improve the accuracy of measurements for demonstration of compliance with various regulations including the dietary supplement current good manufacturing practices (cGMPs). Exercise J of this program offered the opportunity for laboratories to assess their in-house measurements of nutritional elements (Ca, Mg, and Zn), contaminants (arsenic and aflatoxins), water-soluble vitamins (vitamins $B_{5}$ and $B_{6}$ ), fat-soluble vitamins (vitamin $E$ ), fatty acids, isoflavones, and botanical authenticity/identification in foods and/or botanical dietary supplement ingredients and finished products.
\end{abstract}

\title{
INTRODUCTION
}

The dietary supplement industry in the US is booming, with two-thirds of adults considering themselves to be supplement users. ${ }^{1}$ Consumption of dietary supplements, which includes vitamin and mineral supplements, represents an annual US expenditure of more than \$25 billion. These figures represent an increasing American and worldwide trend, and as a result, it is critically important that both the quality and safety of these products are verified and maintained.

The Dietary Supplement Health and Education Act of 1994 (DSHEA) amended the Federal Food, Drug, and Cosmetic Act to create the regulatory category called dietary supplements. The DSHEA also gave the FDA authority to write current Good Manufacturing Practices (cGMPs) that require manufacturers to evaluate the identity, purity, and composition of their ingredients and finished products. In addition the DSHEA authorized the establishment of the Office of Dietary Supplements at the National Institutes of Health (NIH ODS). To enable members of the dietary supplements community to improve the accuracy of the measurements required for compliance with these and other regulations, NIST established the Dietary Supplement Laboratory Quality Assurance Program (DSQAP) in collaboration with the NIH ODS in 2007.

The program offers the opportunity for laboratories to assess their in-house measurements of active or marker compounds, nutritional elements, contaminants (toxic elements, pesticides, mycotoxins), and fat- and water-soluble vitamins in foods as well as botanical dietary supplement ingredients and finished products. Reports and certificates of participation are provided and can be used to demonstrate compliance with the cGMPs. In addition, NIST and the DSQAP assist the ODS Analytical Methods and Reference Materials program (AMRM) at the NIH in supporting the development and dissemination of analytical tools and reference materials. In the future, results from DSQAP exercises could be used by ODS to identify problematic matrices and analytes for which an AOAC Official Method of Analysis would benefit the dietary supplement community.

NIST has experience in the administration of quality assurance programs, but the DSQAP takes a unique approach: In other NIST quality assurance programs, a set of analytes is measured repeatedly over time in the same or similar matrices to demonstrate laboratory performance. In contrast, the wide range of matrices and analytes under the "dietary supplement” umbrella means

\footnotetext{
${ }^{1}$ Walsh, T. (2012) Supplement Usage, Consumer Confidence Remain Steady According to New Annual Survey from CRN. Council for Responsible Nutrition, Washington, DC.
} 
that not every laboratory is interested in every sample or analyte. The constantly changing dietary supplement market, and the enormous diversity of finished products, makes repeated determination of a few target compounds in a single matrix of little use to participants. Instead, participating laboratories are interested in testing in-house methods on a wide variety of challenging, real-world matrices to demonstrate that their performance is comparable to that of the community and that their methods provide accurate results. In an area where there are few standard methods, the DSQAP offers a unique tool for assessment of the quality of measurements, provides feedback about performance, and can assist participants in improving laboratory operations.

This report summarizes the results from the tenth exercise of the DSQAP, Exercise J. Fifty-eight laboratories responded to the call for participants distributed in April 2013. Samples were shipped to participants in June 2013, and results were returned to NIST by September 2013. This report contains the final data and information to be disseminated to the participants in February 2014.

\section{OVERVIEW OF DATA TREATMENT AND REPRESENTATION}

\section{$\underline{\text { Statistics }}$}

The individual data table and graphs contain information about the performance of each laboratory relative to that of the other participants in this study and relative to a target around the expected result (if available). The consensus mean and standard deviation are calculated according to the robust algorithm outlined in ISO 13528:2005(E), Annex C. ${ }^{2}$ The algorithm is summarized here in simplified form.

Initial values of the consensus mean, $x^{*}$, and consensus standard deviation, $s^{*}$, are estimated as

$$
\begin{array}{ll}
x^{*}=\text { median of } x_{i} & (i=1,2, \ldots, n) \\
s^{*}=1.483 \times \text { median of }\left|x_{i}-x^{*}\right| & (i=1,2, \ldots, n)
\end{array}
$$

These initial values for $x^{*}$ and $s^{*}$ are updated by first calculating the expanded standard deviation, $\delta$, as

$$
\delta=1.5 \times s^{*} .
$$

Then each $x_{i}$ is compared to the expanded range and adjusted to $x_{i}^{*}$ as described below to reduce the effect of outliers.

$$
\begin{aligned}
& \text { If } x_{i}<x^{*}-\delta \text {, then } x_{i}^{*}=x^{*}-\delta \text {. } \\
& \text { If } x_{i}>x^{*}+\delta \text {, then } x_{i}^{*}=x^{*}+\delta \text {. } \\
& \text { Otherwise, } x_{i}^{*}=x_{i} \text {. }
\end{aligned}
$$

New values of $x^{*}, s^{*}$, and $\delta$ are calculated iteratively until the process converges. Convergence is taken as no change from one iteration to the next in the third significant figure of $s^{*}$ and in the equivalent digit in $x^{*}$ :

\footnotetext{
${ }^{2}$ ISO 13528:2005(E), Statistical methods for use in proficiency testing by interlaboratory comparisons, pp 14-15.
} 


$$
\begin{aligned}
& X^{*}=\frac{\sum_{i=1}^{n} x_{i}^{*}}{n} \\
& S^{*}=1.134 \times \sqrt{\frac{\sum_{i=1}^{n}\left(x_{i}^{*}-x^{*}\right)}{n-1}} .
\end{aligned}
$$

Individual Data Table

The data in this table is individualized to each participating laboratory and is provided to allow participants to directly compare their data to the summary statistics (consensus or community data as well as NIST certified, reference, or estimated values). The upper left of the data table includes the randomized laboratory code. Tables included in this report are generated using NIST data to protect the identity and performance of participants.

Section 1 of the data table contains the laboratory results as reported, including the mean and standard deviation when multiple values were reported. A blank indicates that NIST does not have data on file for that laboratory for a particular analyte or matrix. An empty box for standard deviation indicates that only a single value was reported and therefore that value was not included in the calculation of the consensus data. ${ }^{2}$

Also in Section 1 are two Z-scores. The first Z-score, $Z_{\text {comm, }}$, is calculated with respect to the community consensus value, using $\mathrm{x}^{*}$ and $\mathrm{s}^{*}$ :

$$
Z_{\text {comm }}=\frac{x_{i}-x *}{s *} .
$$

The second Z-score, $Z_{\text {NIST, }}$ is calculated with respect to the target value (NIST certified, reference, or estimated value), using $\mathrm{x}_{\mathrm{NIST}}$ and $U_{95}$ (the expanded uncertainty) or $\mathrm{s}_{\mathrm{NIST}}$, the standard deviation of NIST measurements:

$$
Z_{N I S T}=\frac{x_{i}-x_{N I S T}}{U_{95}}
$$

or

$$
Z_{N I S T}=\frac{x_{i}-x_{N I S T}}{s_{N I S T}}
$$

The significance of the Z-score is as follows:

- $|\mathrm{Z}|<2$ indicates that the laboratory result is considered to be within the community consensus range (for $\mathrm{Z}_{\text {comm }}$ ) or NIST target range (for $\mathrm{Z}_{\mathrm{NIST}}$ ).

- $2<|Z|<3$ indicates that the laboratory result is considered to be marginally different from the community consensus value (for $\mathrm{Z}_{\text {comm }}$ ) or NIST target value (for $\mathrm{Z}_{\mathrm{NIST}}$ ).

- $|\mathrm{Z}|>3$ indicates that the laboratory result is considered to be significantly different from the community consensus value (for $\mathrm{Z}_{\text {comm }}$ ) or NIST target value (for $\mathrm{Z}_{\mathrm{NIST}}$ ).

Section 2 of the data table contains the community results, including the number of laboratories reporting more than a single value for a given analyte ${ }^{1}$, the mean value determined for each analyte, and a robust estimate of the standard deviation of the reported values. ${ }^{3}$ Consensus means and standard deviations are calculated using the laboratory means; if a laboratory reported

\footnotetext{
${ }^{3}$ ISO 13528:2005(E), Statistical methods for use in proficiency testing by interlaboratory comparisons, Annex C.
} 
a single value, the reported value is not included. ${ }^{1}$ Additional information on calculation of the consensus mean and standard deviation can be found in the previous section.

Section 3 of the data table contains the target values for each analyte. When possible, the target value is a certified or reference value determined at NIST. Certified values and the associated expanded uncertainty $\left(U_{95}\right)$ have been determined with two independent analytical methods at NIST, by collaborating laboratories, or in some combination. Reference values are assigned using NIST values obtained from the average and standard deviation of measurements made using a single analytical method or by measurements obtained from collaborating laboratories. For both certified and reference values, at least six samples have been tested and duplicate preparations from the sample package have been included, allowing the uncertainty to encompass variability due to inhomogeneity within and between packages. For samples in which a NIST certified or reference value is not available, the analytes are measured at NIST using an appropriate method. The NIST-assessed value represents the mean of at least three replicates. For materials acquired from another proficiency testing program, the consensus value and uncertainty from the completed round is used as the target range.

\section{Summary Data Table}

This data table includes a summary of all reported data for a particular analyte in a particular study. Participants can compare the raw data for a single laboratory to data reported by the other participating laboratories or to the consensus data. A blank indicates that the laboratory signed up and received samples for that particular analyte and matrix, but NIST does not have data on file for that laboratory.

\section{Graphs}

Data Summary View (Method Comparison Data Summary View)

In this view, individual laboratory data are plotted with the individual laboratory standard deviation (error bars). Data points that are unfilled represent laboratories that only reported a single value for that analyte and therefore were not included in the consensus mean. The black solid line represents the consensus mean, and the black dotted lines represent the consensus variability calculated as one standard deviation about the consensus mean. Where appropriate, two consensus means may be calculated for the same sample if bimodality is identified in the data. In this case, two consensus means and ranges will be displayed in the data summary view. The gray shaded region represents the target zone for "acceptable” performance, which encompasses the NIST certified, reference, or estimated value bounded by twice its uncertainty $\left(U_{95}\right)$ or standard deviation. For the purpose of the DSQAP, a target range spanning twice the uncertainty in the NIST value is selected because participants are only asked to make a limited number of observations. The size of the y-axis on the data summary view graph represents the consensus mean bounded by $2 \delta$. In this view, the relative locations of individual laboratory data and consensus zones with respect to the target zone can be compared easily. In most cases, the target zone and the consensus zone overlap, which is the expected result. One program goal is to reduce the size of the consensus zone and center the consensus zone about the target value. Analysis of an appropriate reference material as part of a quality control scheme can help to identify sources of bias for laboratories reporting results that are significantly different from the target zone. In the case in which a method comparison is relevant, different colored data points may be used to indicate laboratories that used a specific approach to sample preparation, analytical method, or quantitation. 


\section{Sample/Control Comparison View (Sample/Sample Comparison View)}

In this view, the individual laboratory results for a control (NIST SRM with a certified value) are compared to the results for an unknown (another NIST SRM with a more challenging matrix, a commercial sample, etc.). The error bars represent the individual laboratory standard deviation. The solid red box represents the target zone for the control (x-axis) and unknown sample (yaxis). The dotted blue box represents the consensus zone for the control (x-axis) and the unknown sample (y-axis). The axes of this graph are centered about the consensus mean values for each sample or control, to a limit of zero and twice the consensus mean. Depending on the variability in the data, the axes may be scaled proportionally to better display the individual data points for each laboratory. In some cases, when the consensus and target ranges have limited overlap, the solid red box may only appear partially on the graph. If the variability in the data is high (greater than $100 \%$ RSD), the dotted blue box may also only appear partially on the graph. This view emphasizes trends in the data that may indicate potential calibration issues or method biases. One program goal is to identify such calibration or method biases and assist participants in improving analytical measurement capabilities. In some cases, when two equally challenging materials are provided, the same view (sample/sample comparison) can be helpful in identifying commonalities or differences in the analysis of the two materials.

\section{Composition View}

In this view, used for the aflatoxins in peanut butter study, total composition of the sample is plotted as a function of the measurement of individual components. This view allows comparison of data in which limited statistical information is available as a result of low participation and/or reporting of one data point per sample. This view is also useful in comparison of methods in which a total composition is reported by some laboratories, but individual components are reported by other laboratories. One program goal is to allow laboratories to demonstrate laboratory performance, regardless of the analytical approach.

\section{Bias View}

In this view, used for the aflatoxins in peanut butter study, the laboratory $\mathrm{Z}_{\mathrm{NIST}}$-score is overlaid for each component, and outlying $\mathrm{Z}_{\mathrm{NIST}}$-scores are highlighted in the marginally different range $(2<|\mathrm{Z}|<3)$ in orange, and in the significantly different range $(|\mathrm{Z}|>3)$ in red. This view demonstrates visually which components result in poor $\mathrm{Z}_{\mathrm{NIST}}$-Scores, and allows comparisons between problematic areas as a function of sample type. In an overall composition analysis, this view allows a laboratory to rapidly identify which analyte is resulting in erroneous results. 


\section{NUTRITIONAL ELEMENTS (Ca, Mg, Zn) IN NATURAL AND ENHANCED WATERS}

\section{Study Overview}

In this study, participants were provided with SRM 1643e Trace Elements in Water and commercially available enhanced water. Participants were asked to use in-house analytical methods to determine the mass fractions of three nutritional elements (calcium, magnesium, and zinc) in each of the matrices and report values on an as-received basis.

\section{Sample Information}

Natural Water. Participants were provided with one polyethylene bottle containing $125 \mathrm{~mL}$ of acidified water. Nitric acid is present at a concentration of approximately $0.8 \mathrm{~mol} / \mathrm{L}$ to stabilize the trace elements. Before use, participants were instructed to thoroughly mix the contents of the bottle, and a sample size of at least $1.0 \mathrm{~mL}$ was recommended. Participants were asked to store the material at controlled room temperature, $10{ }^{\circ} \mathrm{C}$ to $30^{\circ} \mathrm{C}$, to prepare three samples, and to report three values from the single bottle provided. Approximate analyte levels were not reported to participants prior to the study. The certified values in SRM 1643e Trace Elements in Water were determined at NIST using inductively coupled plasma mass spectrometry (ICP-MS) and inductively coupled plasma optical emission spectrometry (ICP-OES). The certified values and uncertainties for $\mathrm{Ca}, \mathrm{Mg}$, and $\mathrm{Zn}$ are outlined in the table below.

Enhanced Water. Participants were provided with one $600 \mathrm{~mL}$ bottle of commercially available enhanced water. Before use, participants were instructed to thoroughly mix the contents of the bottle, and a sample size of at least $1.0 \mathrm{~mL}$ was recommended. Participants were asked to store the material at controlled room temperature, $10{ }^{\circ} \mathrm{C}$ to $30^{\circ} \mathrm{C}$, to prepare three samples, and to report three values from the single bottle provided. Approximate analyte levels were not reported to participants prior to the study. Certified values are not available for this material; NIST provided values for $\mathrm{Ca}, \mathrm{Mg}$, and Zn based on triplicate analysis using ICP-OES using standard additions as the method of quantitation. The NIST values in enhanced water are reported in the table below with an estimated relative uncertainty of $5 \%$.

$\begin{array}{ccc}\text { Analyte } & \begin{array}{c}\text { Certified Mass Fraction } \\ \text { in SRM 1643e }(\mu \mathrm{g} / \mathrm{g})\end{array} \\ \mathrm{Ca} & & 31.5 \pm 1.1 \\ \mathrm{Mg} & & 7.84 \pm 0.096 \\ \mathrm{Zn} & 0.0765 \pm 0.0021\end{array}$

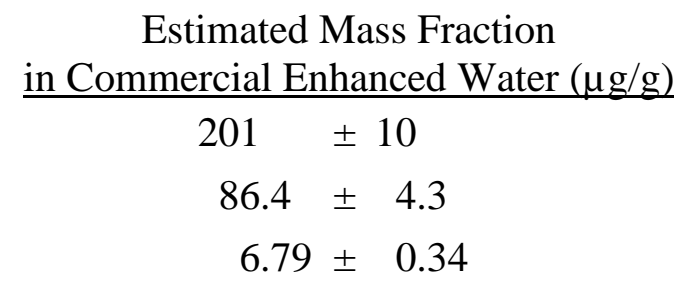

Study Results

- Thirty-five laboratories enrolled in this exercise and received samples. Twenty-nine laboratories reported results for calcium and magnesium (83 \% participation). Twentysix to 29 laboratories reported results for zinc (74\% to $83 \%$ participation).

- The consensus means for calcium and magnesium in both materials and for zinc in the enhanced water were within the target range with an acceptable variability (5\% to $14 \%$ relative standard deviation (RSD)).

- The consensus mean for zinc in SRM 1643e was above the target range with a high variability (59\% RSD).

- Zinc in the natural water is two orders of magnitude lower than that of the enhanced water, making this material near the method detection limit (MDL) or limit of 
quantitation (LOQ) for some participants. Measuring near the MDL or LOQ can give erroneous values, either high or low. Figure 15 demonstrates this by showing the consensus zone more tightly surrounding the target zone for enhanced water than for the natural water.

- A majority of the laboratories reported using either open-beaker digestion (34\%) or microwave digestion (41\%) for sample preparation. The remaining laboratories reported using hot block digestion, dilution, or other methods (14\%).

- A majority of the laboratories reported using either ICP-OES (55\%) or ICP-MS (41 \%) as their analytical method. One laboratory reported using atomic absorption spectroscopy (AAS).

Technical Recommendations

The following recommendations are based on results obtained by the participants in this study.

- Both water samples should have been straightforward to digest; no trends were observed based on either the sample preparation method or analytical method used.

- The most likely source of error in this study is related to construction of the calibration curves. To avoid calibration problems, be sure to

o Include the lowest and highest expected solution concentrations, plus one or two intermediate concentration points in the calibration curve.

o Ensure that the calibration curve is linear and surrounds expected sample concentrations following digestion and/or dilution. Samples should not go beyond the linear range of the calibration curve resulting in extrapolation of calibration curves leading to false values.

o Use a sufficient number of blanks to accurately determine MDL and LOQ.

- Run a quality control sample (either in-house or a commercially available reference material) of known concentration to ensure that your method is performing as expected.

- Double-check all calculations; miscalculations and reporting of wrong units were a cause for some errors. 
Table 1. Individual data summary table (NIST) for nutritional elements in water.

\section{National Institute of Standards \& Technology}

\begin{tabular}{ccc} 
& Lab Code: & NIST \\
\hline Analyte & Sample & Units \\
\hline $\mathrm{Ca}$ & Natural Water & $\mu \mathrm{g} / \mathrm{g}$ \\
$\mathrm{Ca}$ & Enhanced Water & $\mu \mathrm{g} / \mathrm{g}$ \\
\hline $\mathrm{Mg}$ & Natural Water & $\mu \mathrm{g} / \mathrm{g}$ \\
$\mathrm{Mg}$ & Enhanced Water & $\mu \mathrm{g} / \mathrm{g}$ \\
\hline $\mathrm{Zn}$ & Natural Water & $\mu \mathrm{g} / \mathrm{g}$ \\
$\mathrm{Zn}$ & Enhanced Water & $\mu \mathrm{g} / \mathrm{g}$ \\
\hline
\end{tabular}

Exercise J - May 2013 - Nutritional Elements

\begin{tabular}{cccc}
\multicolumn{4}{c}{ 1. Your Results } \\
\hline $\mathrm{x}_{\mathrm{i}}$ & $\mathrm{s}_{\mathrm{i}}$ & $\mathrm{Z}_{\text {comm }}$ & $\mathrm{Z}_{\mathrm{NIST}}$ \\
\hline 31.5 & 1.1 & 0.0 & 0.0 \\
201 & 3 & -0.3 & 0.0 \\
\hline 7.84 & 0.10 & -0.2 & 0.0 \\
86.4 & 0.1 & 0.0 & 0.0 \\
\hline 0.0765 & 0.0021 & -0.6 & 0.0 \\
6.79 & 0.02 & -0.3 & 0.0 \\
\hline
\end{tabular}

\section{Community Results}

\begin{tabular}{ccc}
\hline $\mathrm{N}$ & $\mathrm{x}^{*}$ & $\mathrm{~s}^{*}$ \\
\hline 29 & 31.4 & 3.2 \\
29 & 204 & 10 \\
\hline 29 & 7.92 & 0.45 \\
29 & 86.4 & 5.1 \\
\hline 26 & 0.1160 & 0.0679 \\
29 & 7.09 & 0.99 \\
\hline
\end{tabular}

$\mathrm{N}$ Number of quantitative values reported

$\mathrm{x}^{*}$ Robust mean of reported values

s* Robust standard deviation$$
\text { Robust standard deviation }
$$

\begin{tabular}{cc}
\multicolumn{2}{c}{ 3. Target } \\
\hline $\mathrm{X}_{\mathrm{NIST}}$ & $U_{95}$ \\
\hline 31.5 & 1.1 \\
201 & 10 \\
\hline 7.84 & 0.10 \\
86.4 & 4.3 \\
\hline 0.0765 & 0.0021 \\
6.79 & 0.34 \\
\hline
\end{tabular}

$\mathrm{X}_{\mathrm{NIST}} \mathrm{NIST}$-assessed value

$U_{95} \pm 95 \%$ confidence interval about the assessed value or standard deviation $\left(\mathrm{s}_{\mathrm{NIST}}\right)$

$\mathrm{Z}_{\mathrm{NIST}} \mathrm{Z}$-score with respect to NIST value 
Table 2. Data summary table for calcium in natural and enhanced waters.

\begin{tabular}{|c|c|c|c|c|c|c|c|c|c|c|c|}
\hline & \multirow[b]{3}{*}{ Lab } & \multicolumn{10}{|c|}{ Calcium } \\
\hline & & \multicolumn{5}{|c|}{ SRM 1643e Trace Elements in Water ( $\mu \mathrm{g} / \mathrm{g})$} & \multicolumn{5}{|c|}{ Enhanced Water $(\mu \mathrm{g} / \mathrm{g})$} \\
\hline & & A & B & C & Avg & SD & A & B & C & Avg & SD \\
\hline \multirow{36}{*}{ 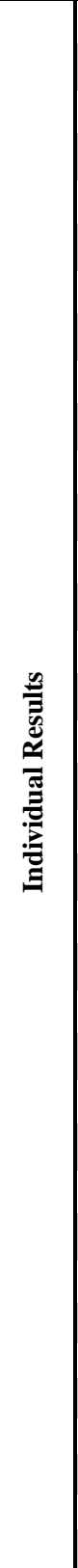 } & NIST & & & & 31.5 & 1.1 & 198 & 202 & 203 & 201 & 3 \\
\hline & J002 & 4.2 & 6.4 & 2.6 & 4.4 & 1.9 & 11 & 17 & 15 & 14 & 3 \\
\hline & J006 & 31.0 & 30.9 & 31.1 & 31.0 & 0.1 & 229 & 223 & 227 & 226 & 3 \\
\hline & J007 & 29.2 & 28.9 & 28.8 & 29.0 & 0.2 & 194 & 196 & 196 & 195 & 1 \\
\hline & J008 & 30.8 & 28.9 & 29.9 & 29.9 & 1.0 & 190 & 190 & 191 & 190 & 1 \\
\hline & J009 & & & & & & & & & & \\
\hline & J014 & 34.4 & 34.0 & 33.2 & 33.9 & 0.6 & 211 & 205 & 210 & 209 & 3 \\
\hline & J016 & 30.9 & 30.7 & 32.0 & 31.2 & 0.7 & 203 & 203 & 197 & 201 & 3 \\
\hline & J017 & 28.7 & 28.2 & 28.3 & 28.4 & 0.3 & 210 & 208 & 210 & 209 & 1 \\
\hline & J018 & & & & & & & & & & \\
\hline & J019 & 2679.3 & 2475.3 & 2497.2 & 2550.6 & 112.0 & 240 & 229 & 253 & 241 & 12 \\
\hline & J020 & 44.8 & 43.8 & 42.9 & 43.8 & 1.0 & 215 & 219 & 215 & 216 & 2 \\
\hline & J021 & & & & & & & & & & \\
\hline & J022 & 32.8 & 30.4 & 31.2 & 31.5 & 1.2 & 195 & 190 & 201 & 195 & 5 \\
\hline & J024 & 34.8 & 35.0 & 34.8 & 34.9 & 0.1 & 219 & 230 & 216 & 222 & 7 \\
\hline & J025 & 55.4 & 55.2 & 54.4 & 55.0 & 0.5 & 199 & 200 & 204 & 201 & 3 \\
\hline & J026 & 29.1 & 28.5 & 31.1 & 29.6 & 1.3 & 187 & 202 & 201 & 197 & 8 \\
\hline & J029 & 31.4 & 31.4 & 30.5 & 31.1 & 0.5 & 204 & 205 & 209 & 206 & 3 \\
\hline & J031 & 30.0 & 30.0 & 30.0 & 30.0 & 0.0 & 204 & 204 & 202 & 203 & 1 \\
\hline & J033 & & & & & & & & & & \\
\hline & J034 & 24.4 & 24.7 & 24.9 & 24.7 & 0.3 & 209 & 208 & 207 & 208 & 1 \\
\hline & J036 & 31.5 & 32.7 & 34.1 & 32.8 & 1.3 & 199 & 195 & 200 & 198 & 3 \\
\hline & J037 & 28.7 & 27.9 & 28.0 & 28.2 & 0.4 & 204 & 203 & 206 & 205 & 2 \\
\hline & J038 & 28.4 & 25.7 & 27.1 & 27.1 & 1.3 & 122 & 140 & 218 & 160 & 51 \\
\hline & J039 & & & & & & & & & & \\
\hline & J041 & & & & & & & & & & \\
\hline & J047 & 32.6 & 32.5 & 32.5 & 32.5 & 0.1 & 203 & 204 & 204 & 204 & 1 \\
\hline & J048 & 32.1 & 32.3 & 32.2 & 32.2 & 0.1 & 203 & 203 & 204 & 203 & 1 \\
\hline & J051 & 29.7 & 30.0 & 29.8 & 29.8 & 0.1 & 201 & 201 & 202 & 201 & 0 \\
\hline & J052 & 44.8 & 46.5 & 39.0 & 43.4 & 3.9 & 270 & 272 & 242 & 262 & 17 \\
\hline & J053 & 31.9 & 32.1 & 30.6 & 31.5 & 0.8 & 198 & 192 & 206 & 199 & 7 \\
\hline & J054 & 28.1 & 28.9 & 28.3 & 28.4 & 0.4 & 196 & 204 & 195 & 198 & 5 \\
\hline & J055 & 33.2 & 32.5 & 32.6 & 32.8 & 0.4 & 199 & 199 & 199 & 199 & 0 \\
\hline & J056 & 32.0 & 31.0 & 31.0 & 31.3 & 0.6 & 210 & 200 & 210 & 207 & 6 \\
\hline & J057 & 30.9 & 29.7 & 29.6 & 30.1 & 0.7 & 201 & 204 & 203 & 202 & 2 \\
\hline & $\mathrm{J} 060$ & 31.5 & 31.7 & 31.6 & 31.6 & 0.1 & 207 & 206 & 206 & 206 & 1 \\
\hline | & & \multicolumn{3}{|c|}{$\begin{array}{l}\text { Consensus Mean } \\
\text { Consensus Standard Deviation } \\
\text { Maximum } \\
\text { Minimum } \\
\text { N }\end{array}$} & $\begin{array}{c}31.4 \\
3.2 \\
2550.6 \\
4.4 \\
29\end{array}$ & & \multicolumn{3}{|c|}{$\begin{array}{l}\text { Consensus M } \\
\text { Consensus St } \\
\text { Maximum } \\
\text { Minimum } \\
\mathrm{N}\end{array}$} & $\begin{array}{c}204 \\
10 \\
262 \\
14 \\
29\end{array}$ & \\
\hline
\end{tabular}


Table 3. Data summary table for magnesium in natural and enhanced waters.

\begin{tabular}{|c|c|c|c|c|c|c|c|c|c|c|c|}
\hline & \multirow[b]{3}{*}{ Lab } & \multicolumn{10}{|c|}{ Magnesium } \\
\hline & & \multicolumn{5}{|c|}{ SRM 1643e Trace Elements in Water $(\mu \mathrm{g} / \mathrm{g})$} & \multicolumn{5}{|c|}{ Enhanced Water $(\mu \mathrm{g} / \mathrm{g})$} \\
\hline & & A & B & C & Avg & SD & A & B & C & Avg & SD \\
\hline \multirow{36}{*}{ 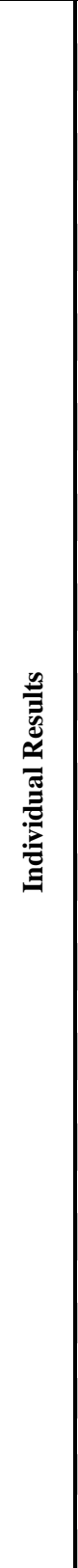 } & NIST & & & & 7.84 & 0.10 & 86.4 & 86.6 & 86.4 & 86.4 & 0.1 \\
\hline & J002 & 6.61 & 6.35 & 6.80 & 6.59 & 0.22 & 72.9 & 71.8 & 72.7 & 72.5 & 0.6 \\
\hline & J006 & 7.82 & 7.84 & 7.80 & 7.82 & 0.02 & 84.6 & 87.3 & 85.7 & 85.9 & 1.4 \\
\hline & J007 & 7.64 & 7.52 & 7.60 & 7.59 & 0.06 & 86.3 & 86.8 & 88.7 & 87.3 & 1.3 \\
\hline & J008 & 7.93 & 7.67 & 7.82 & 7.81 & 0.13 & 81.3 & 82.1 & 82.0 & 81.8 & 0.4 \\
\hline & J009 & & & & & & & & & & \\
\hline & J014 & 7.69 & 7.90 & 7.89 & 7.83 & 0.12 & 91.2 & 89.1 & 90.5 & 90.3 & 1.1 \\
\hline & J016 & 8.33 & 8.14 & 8.24 & 8.24 & 0.10 & 91.5 & 91.7 & 90.0 & 91.1 & 0.9 \\
\hline & J017 & 8.00 & 7.90 & 7.89 & 7.93 & 0.06 & 90.8 & 89.6 & 90.3 & 90.2 & 0.6 \\
\hline & J018 & & & & & & & & & & \\
\hline & J019 & 835.20 & 812.00 & 819.10 & 822.10 & 11.89 & 107.4 & 94.0 & 90.7 & 97.4 & 8.8 \\
\hline & J020 & 12.60 & 12.60 & 12.20 & 12.47 & 0.23 & 91.5 & 82.6 & 92.8 & 89.0 & 5.6 \\
\hline & J021 & & & & & & & & & & \\
\hline & J022 & 8.33 & 7.85 & 8.34 & 8.17 & 0.28 & 94.3 & 95.1 & 92.2 & 93.8 & 1.5 \\
\hline & J024 & 8.72 & 8.62 & 8.60 & 8.65 & 0.06 & 89.3 & 97.0 & 88.4 & 91.6 & 4.7 \\
\hline & J025 & 7.70 & 7.70 & 7.60 & 7.67 & 0.06 & 85.3 & 86.2 & 87.8 & 86.4 & 1.3 \\
\hline & J026 & 7.31 & 7.17 & 7.84 & 7.44 & 0.35 & 80.1 & 85.3 & 85.0 & 83.5 & 2.9 \\
\hline & J029 & 7.87 & 7.72 & 7.37 & 7.65 & 0.26 & 83.1 & 84.5 & 85.6 & 84.4 & 1.3 \\
\hline & J031 & 8.00 & 8.00 & 8.00 & 8.00 & 0.00 & 86.0 & 86.0 & 85.0 & 85.7 & 0.6 \\
\hline & J033 & & & & & & & & & & \\
\hline & J034 & 7.72 & 7.80 & 7.83 & 7.78 & 0.06 & 82.5 & 81.8 & 82.1 & 82.1 & 0.4 \\
\hline & J036 & 8.16 & 8.58 & 10.80 & 9.18 & 1.42 & 89.1 & 87.9 & 88.6 & 88.5 & 0.6 \\
\hline & J037 & 7.70 & 7.48 & 7.51 & 7.56 & 0.12 & 89.5 & 88.4 & 89.5 & 89.1 & 0.7 \\
\hline & J038 & 7.06 & 6.41 & 6.80 & 6.76 & 0.33 & 44.6 & 55.2 & 90.9 & 63.6 & 24.2 \\
\hline & J039 & & & & & & & & & & \\
\hline & J041 & & & & & & & & & & \\
\hline & J047 & 8.39 & 8.49 & 8.39 & 8.42 & 0.06 & 88.8 & 89.0 & 89.6 & 89.1 & 0.4 \\
\hline & J048 & 8.00 & 8.10 & 8.05 & 8.05 & 0.05 & 82.5 & 82.3 & 82.1 & 82.3 & 0.2 \\
\hline & J051 & 7.73 & 7.82 & 7.76 & 7.77 & 0.04 & 87.5 & 87.1 & 87.8 & 87.5 & 0.4 \\
\hline & J052 & 8.06 & 8.07 & 8.11 & 8.08 & 0.02 & 75.7 & 75.7 & 75.5 & 75.6 & 0.1 \\
\hline & J053 & 7.94 & 8.20 & 7.68 & 7.94 & 0.26 & 85.7 & 82.0 & 80.8 & 82.8 & 2.6 \\
\hline & J054 & 7.54 & 7.52 & 7.61 & 7.56 & 0.05 & 86.3 & 88.2 & 89.1 & 87.9 & 1.4 \\
\hline & J055 & 7.51 & 7.30 & 7.29 & 7.37 & 0.12 & 82.3 & 82.2 & 82.1 & 82.2 & 0.1 \\
\hline & J056 & 8.20 & 7.80 & 8.20 & 8.07 & 0.23 & 95.0 & 92.0 & 94.0 & 93.7 & 1.5 \\
\hline & J057 & 8.04 & 7.88 & 7.97 & 7.96 & 0.08 & 86.1 & 85.6 & 85.6 & 85.8 & 0.3 \\
\hline & $\mathrm{J} 060$ & 7.30 & 8.10 & 8.07 & 7.82 & 0.45 & 86.6 & 86.6 & 86.9 & 86.7 & 0.2 \\
\hline | & & \multicolumn{3}{|c|}{$\begin{array}{l}\text { Consensus Mean } \\
\text { Consensus Standard Deviation } \\
\text { Maximum } \\
\text { Minimum } \\
\text { N }\end{array}$} & $\begin{array}{c}7.92 \\
0.45 \\
822.10 \\
6.59 \\
29\end{array}$ & & \multicolumn{3}{|c|}{$\begin{array}{l}\text { Consensus } \\
\text { Consensus } \\
\text { Maximum } \\
\text { Minimum } \\
\mathrm{N}\end{array}$} & $\begin{array}{c}86.4 \\
5.1 \\
97.4 \\
63.6 \\
29\end{array}$ & \\
\hline
\end{tabular}


Table 4. Data summary table for zinc in natural and enhanced waters.

\begin{tabular}{|c|c|c|c|c|c|c|c|c|c|c|c|}
\hline & \multirow[b]{3}{*}{ Lab } & \multicolumn{10}{|c|}{ Zinc } \\
\hline & & \multicolumn{5}{|c|}{ SRM 1643e Trace Elements in Water $(\mu \mathrm{g} / \mathrm{g})$} & \multicolumn{5}{|c|}{ Enhanced Water $(\mu \mathrm{g} / \mathrm{g})$} \\
\hline & & A & B & $\mathbf{C}$ & Avg & SD & $\mathbf{A}$ & B & $\mathbf{C}$ & Avg & SD \\
\hline \multirow{36}{*}{ 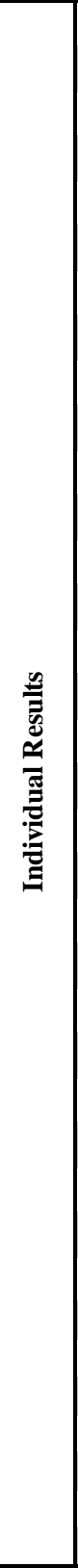 } & NIST & & & & 0.0765 & 0.0021 & 6.77 & 6.82 & 6.79 & 6.79 & 0.02 \\
\hline & J002 & 842.5982 & 812.8184 & 1099.6997 & 918.3721 & 157.7387 & 824.04 & 842.77 & 862.72 & 843.18 & 19.34 \\
\hline & J006 & 0.0817 & 0.0797 & 0.0396 & 0.0670 & 0.0238 & 8.19 & 8.63 & 8.27 & 8.37 & 0.23 \\
\hline & J007 & 0.0710 & 0.0710 & 0.0700 & 0.0707 & 0.0006 & 6.40 & 6.43 & 6.55 & 6.46 & 0.08 \\
\hline & J008 & 0.1558 & 0.1578 & 0.1698 & 0.1611 & 0.0076 & 6.32 & 6.27 & 6.34 & 6.31 & 0.04 \\
\hline & J009 & & & & & & & & & & \\
\hline & J014 & 0.0647 & 0.0660 & 0.0647 & 0.0651 & 0.0008 & 5.84 & 5.83 & 5.98 & 5.88 & 0.08 \\
\hline & J016 & 0.0893 & 0.0896 & 0.0848 & 0.0879 & 0.0027 & 6.71 & 6.71 & 6.70 & 6.71 & 0.01 \\
\hline & J017 & 0.0800 & 0.0755 & 0.0764 & 0.0773 & 0.0024 & 6.88 & 7.02 & 6.93 & 6.94 & 0.07 \\
\hline & J018 & & & & & & & & & & \\
\hline & J019 & 13.0000 & 11.7000 & 11.8000 & 12.1667 & 0.7234 & & 16.30 & 15.10 & 15.70 & 0.85 \\
\hline & J020 & 0.3400 & 0.2000 & 0.1300 & 0.2233 & 0.1069 & 6.80 & 6.60 & 6.50 & 6.63 & 0.15 \\
\hline & J021 & & & & & & & & & & \\
\hline & J022 & 0.0770 & 0.0710 & 0.0750 & 0.0743 & 0.0031 & 6.07 & 6.24 & 6.21 & 6.17 & 0.09 \\
\hline & J024 & 0.0992 & 0.0948 & 0.1130 & 0.1023 & 0.0095 & 6.76 & 7.73 & 6.88 & 7.12 & 0.53 \\
\hline & J025 & 1.5691 & 1.5741 & 1.5496 & 1.5643 & 0.0129 & 6.52 & 6.90 & 6.54 & 6.65 & 0.21 \\
\hline & J026 & 0.1868 & 0.1776 & 0.1813 & 0.1819 & 0.0046 & 10.53 & 8.90 & 8.41 & 9.28 & 1.11 \\
\hline & J029 & 0.0850 & 0.0820 & 0.0900 & 0.0857 & 0.0040 & 6.93 & 7.07 & 6.67 & 6.89 & 0.20 \\
\hline & J031 & & & & & & 7.00 & 7.00 & 7.00 & 7.00 & 0.00 \\
\hline & J033 & & & & & & & & & & \\
\hline & J034 & & & & & & 6.97 & 6.91 & 6.91 & 6.93 & 0.03 \\
\hline & J036 & & & & & & 7.11 & 7.16 & 7.17 & 7.15 & 0.03 \\
\hline & J037 & 0.0711 & 0.0714 & 0.0710 & 0.0712 & 0.0002 & 6.53 & 6.42 & 6.50 & 6.48 & 0.06 \\
\hline & J038 & 0.3300 & 0.1800 & 0.1800 & 0.2300 & 0.0866 & 8.40 & 8.51 & 9.06 & 8.66 & 0.35 \\
\hline & J039 & & & & & & & & & & \\
\hline & J041 & & & & & & & & & & \\
\hline & J047 & 0.0770 & 0.0760 & 0.0780 & 0.0770 & 0.0010 & 6.79 & 6.82 & 6.86 & 6.82 & 0.04 \\
\hline & J048 & 0.0750 & 0.0760 & 0.0770 & 0.0760 & 0.0010 & 6.94 & 7.02 & 7.00 & 6.99 & 0.04 \\
\hline & J051 & 0.0776 & 0.0773 & 0.0778 & 0.0776 & 0.0002 & 6.70 & 6.69 & 6.69 & 6.69 & 0.01 \\
\hline & J052 & 0.0610 & 0.0610 & 0.0620 & 0.0613 & 0.0006 & 10.37 & 10.57 & 10.61 & 10.52 & 0.13 \\
\hline & J053 & 0.0920 & 0.0860 & 0.0970 & 0.0917 & 0.0055 & 5.91 & 6.06 & 6.46 & 6.14 & 0.28 \\
\hline & J054 & 0.1454 & 0.1453 & 0.1454 & 0.1454 & 0.0001 & 5.94 & 6.20 & 6.31 & 6.15 & 0.19 \\
\hline & J055 & 0.0998 & 0.0976 & 0.0977 & 0.0983 & 0.0012 & 7.83 & 7.97 & 7.90 & 7.90 & 0.07 \\
\hline & J056 & 0.0640 & 0.0640 & 0.0640 & 0.0640 & 0.0000 & 6.20 & 6.00 & 6.00 & 6.07 & 0.12 \\
\hline & J057 & 0.0835 & 0.0775 & 0.0795 & 0.0802 & 0.0031 & 7.03 & 7.07 & 7.01 & 7.03 & 0.03 \\
\hline & $\mathrm{J} 060$ & 0.0660 & 0.0660 & 0.0660 & 0.0660 & 0.0000 & 6.59 & 6.57 & 6.58 & 6.58 & 0.01 \\
\hline |气 & & $\begin{array}{l}\text { Consensus } \\
\text { Consensus } \\
\text { Maximum } \\
\text { Minimum } \\
\mathrm{N}\end{array}$ & $\begin{array}{l}\text { Mean } \\
\text { Standard D }\end{array}$ & eviation & $\begin{array}{c}0.1160 \\
0.0679 \\
918.3721 \\
0.0613 \\
26\end{array}$ & & $\begin{array}{l}\text { Consensus } \\
\text { Consensus } \\
\text { Maximum } \\
\text { Minimum } \\
\mathrm{N}\end{array}$ & $\begin{array}{l}\text { Yean } \\
\text { tandard I }\end{array}$ & iation & $\begin{array}{c}7.09 \\
0.99 \\
843.18 \\
5.88 \\
29\end{array}$ & \\
\hline
\end{tabular}




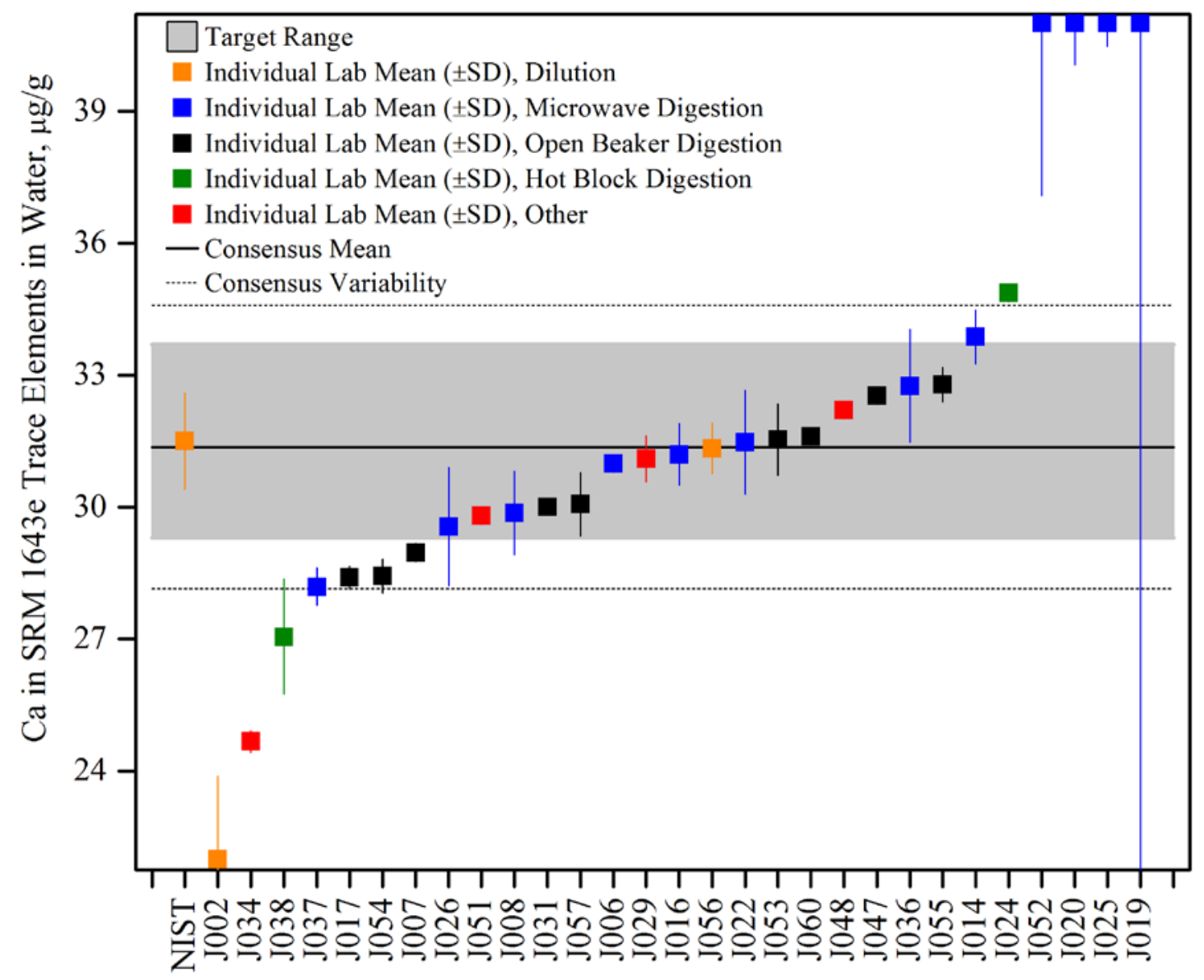

Figure 1. Calcium in SRM 1643e Trace Elements in Water (data summary view - digestion method). In this view, individual laboratory data are plotted with the individual laboratory standard deviation (error bars). The color of the data point represents the sample preparation (digestion) procedure employed. The black solid line represents the consensus mean, and the black dotted lines represent the consensus variability calculated as one standard deviation about the consensus mean. The gray shaded region represents the target zone for "acceptable" performance, which encompasses the NIST certified value bounded by twice its uncertainty $\left(U_{95}\right)$. 


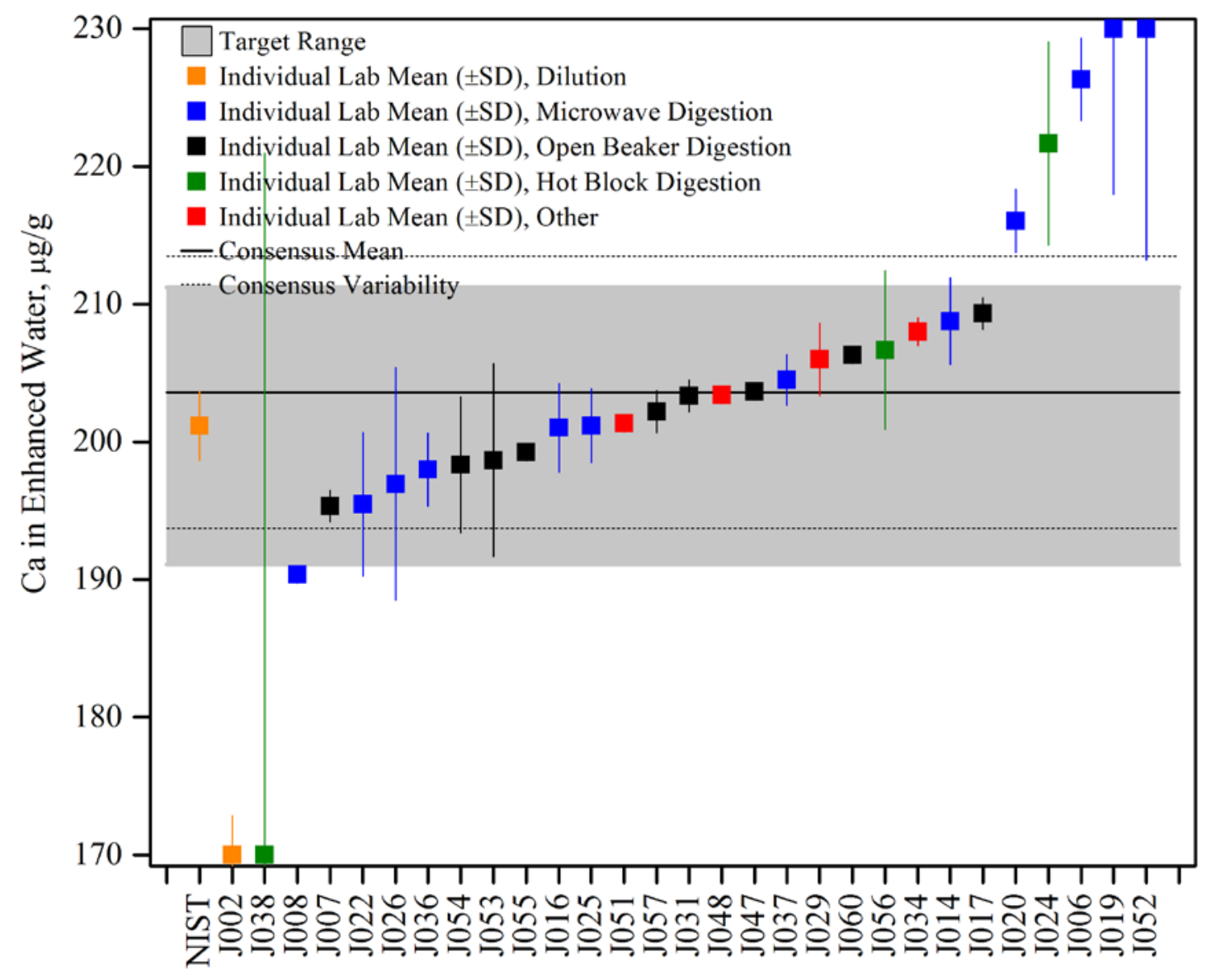

Figure 2. Calcium in enhanced water (data summary view - digestion method). In this view, individual laboratory data are plotted with the individual laboratory standard deviation (error bars). The color of the data point represents the sample preparation (digestion) procedure employed. The black solid line represents the consensus mean, and the black dotted lines represent the consensus variability calculated as one standard deviation about the consensus mean. The gray shaded region represents the target zone for "acceptable" performance, which encompasses the NIST value determined in triplicate by ICP-OES, bounded by an estimated relative uncertainty of $5 \%$. 


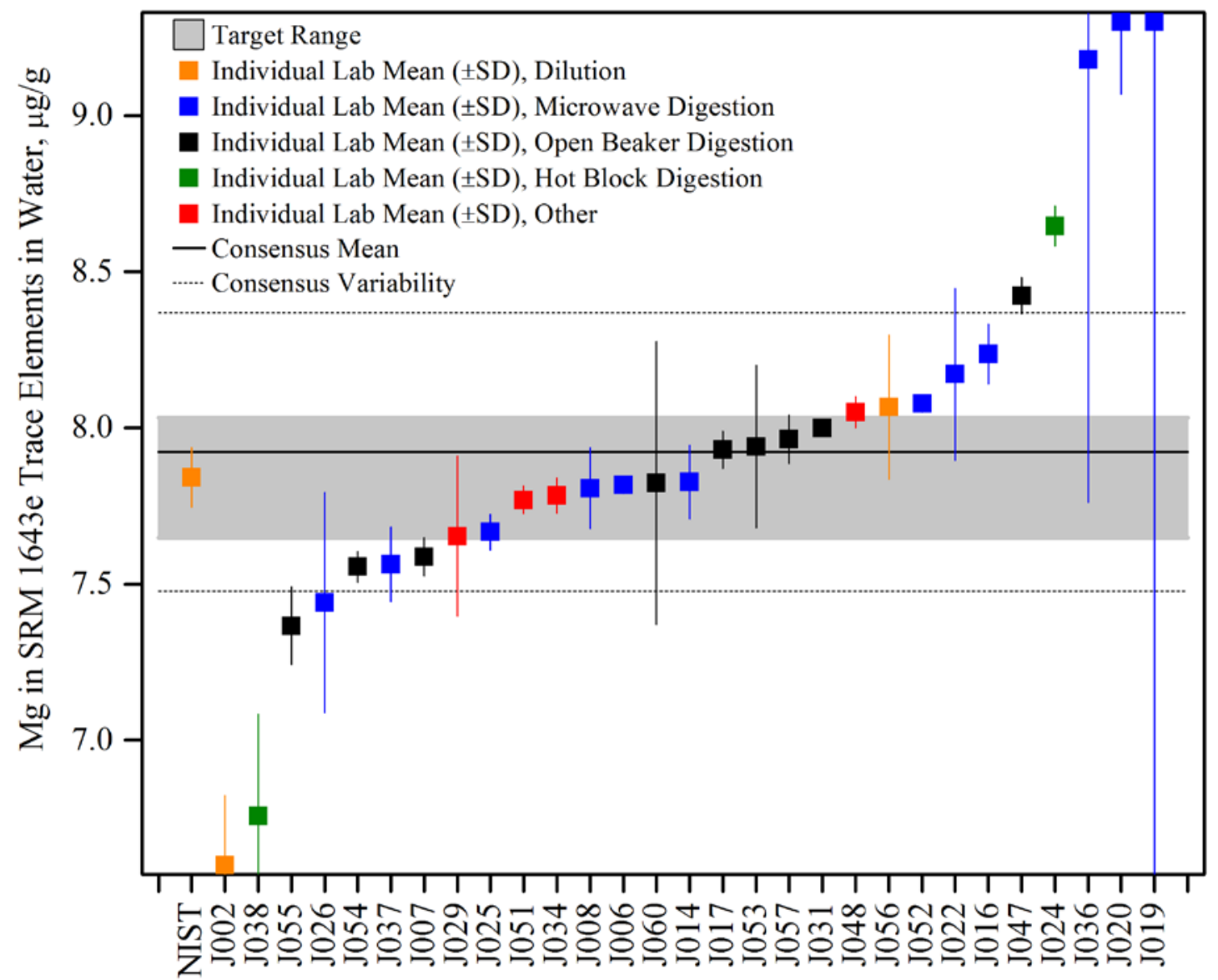

Figure 3. Magnesium in SRM 1643e Trace Elements in Water (data summary view - digestion method). In this view, individual laboratory data are plotted with the individual laboratory standard deviation (error bars). The color of the data point represents the sample preparation (digestion) procedure employed. The black solid line represents the consensus mean, and the black dotted lines represent the consensus variability calculated as one standard deviation about the consensus mean. The gray shaded region represents the target zone for "acceptable" performance, which encompasses the NIST certified value bounded by twice its uncertainty $\left(U_{95}\right)$. 


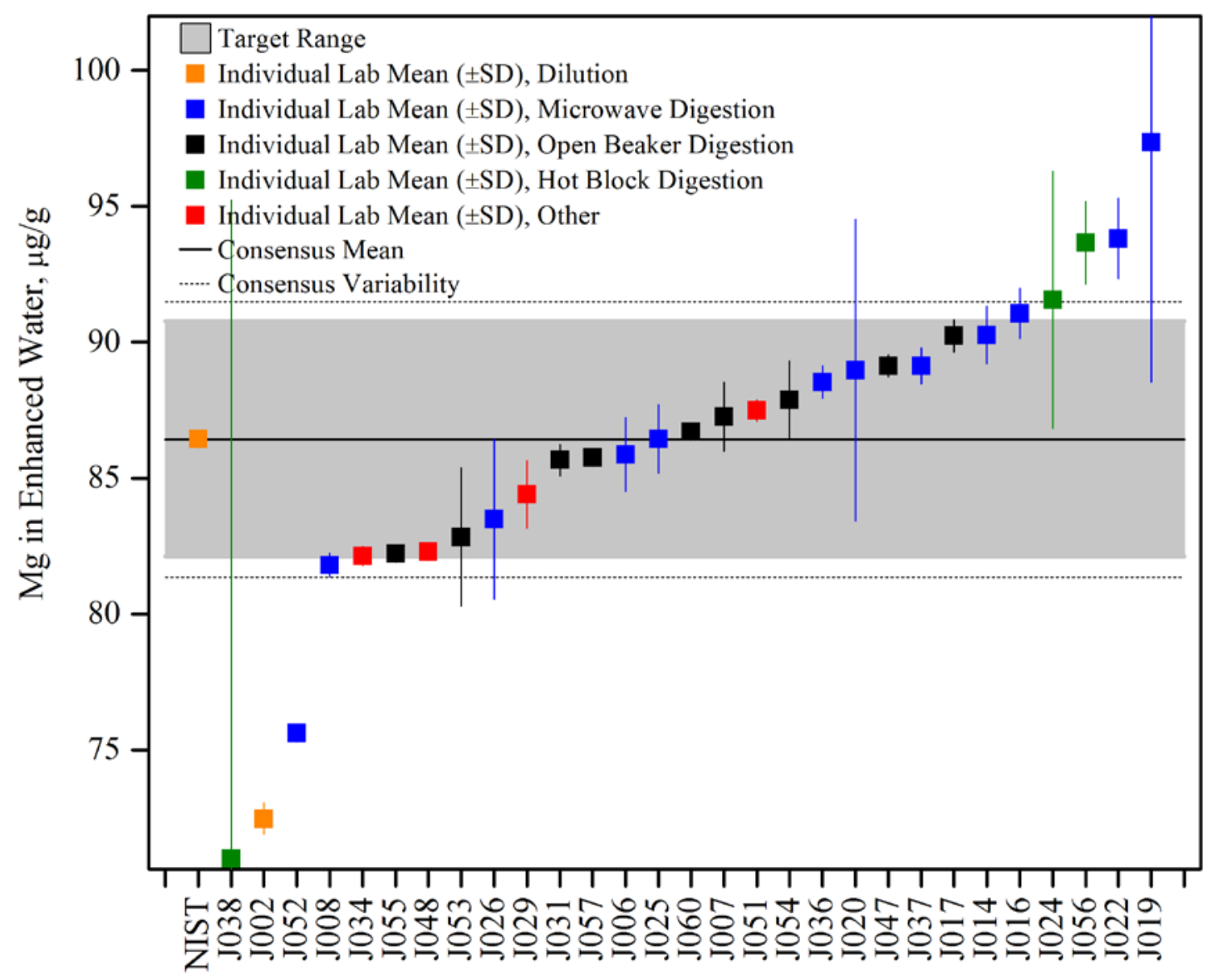

Figure 4. Magnesium in enhanced water (data summary view - digestion method). In this view, individual laboratory data are plotted with the individual laboratory standard deviation (error bars). The color of the data point represents the sample preparation (digestion) procedure employed. The black solid line represents the consensus mean, and the black dotted lines represent the consensus variability calculated as one standard deviation about the consensus mean. The gray shaded region represents the target zone for "acceptable" performance, which encompasses the NIST value determined in triplicate by ICP-OES, bounded by an estimated relative uncertainty of $5 \%$. 


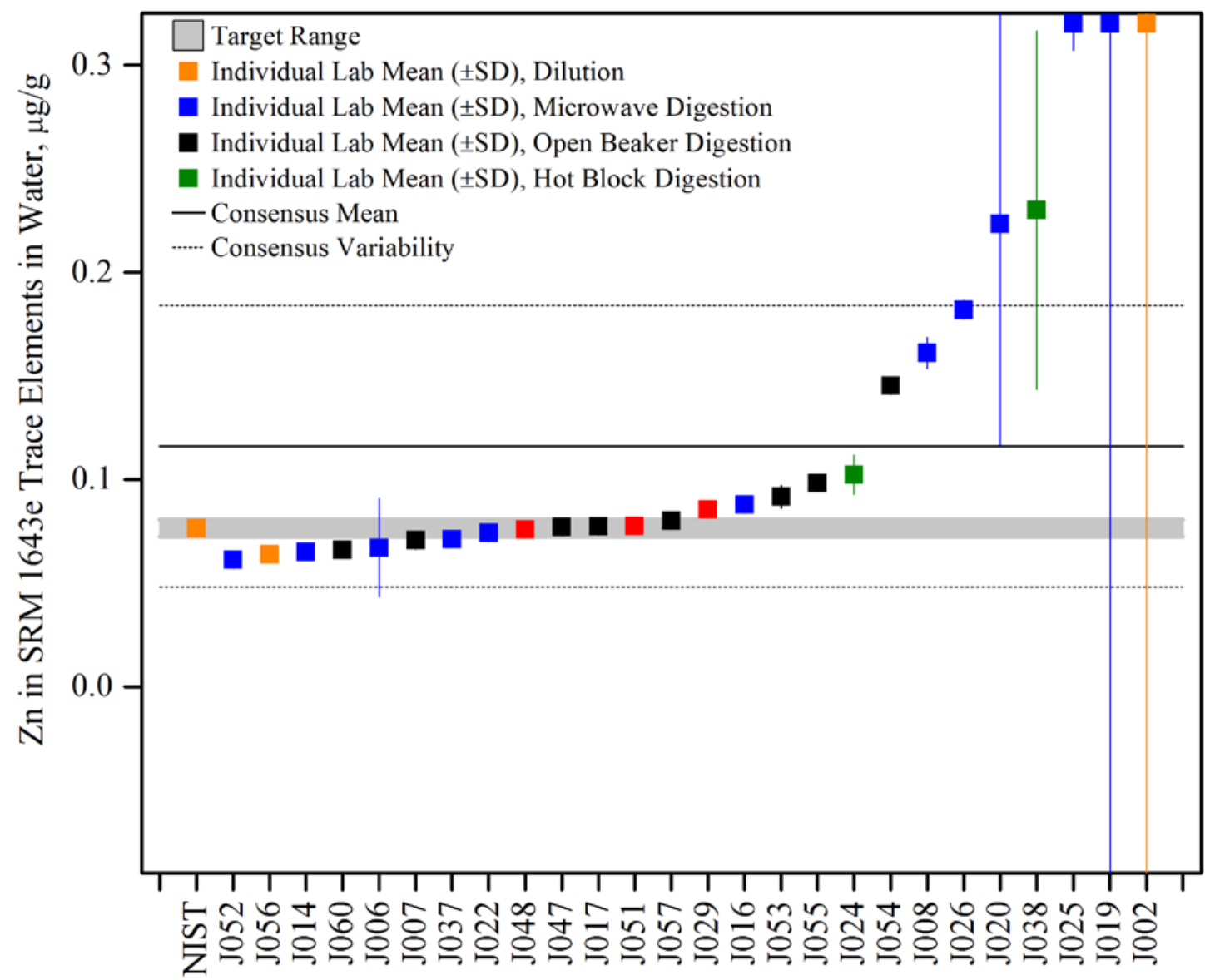

Figure 5. Zinc in SRM 1643e Trace Elements in Water (data summary view - digestion method). In this view, individual laboratory data are plotted with the individual laboratory standard deviation (error bars). The color of the data point represents the sample preparation (digestion) procedure employed. The black solid line represents the consensus mean, and the black dotted lines represent the consensus variability calculated as one standard deviation about the consensus mean. The gray shaded region represents the target zone for "acceptable" performance, which encompasses the NIST certified value bounded by twice its uncertainty $\left(U_{95}\right)$. 


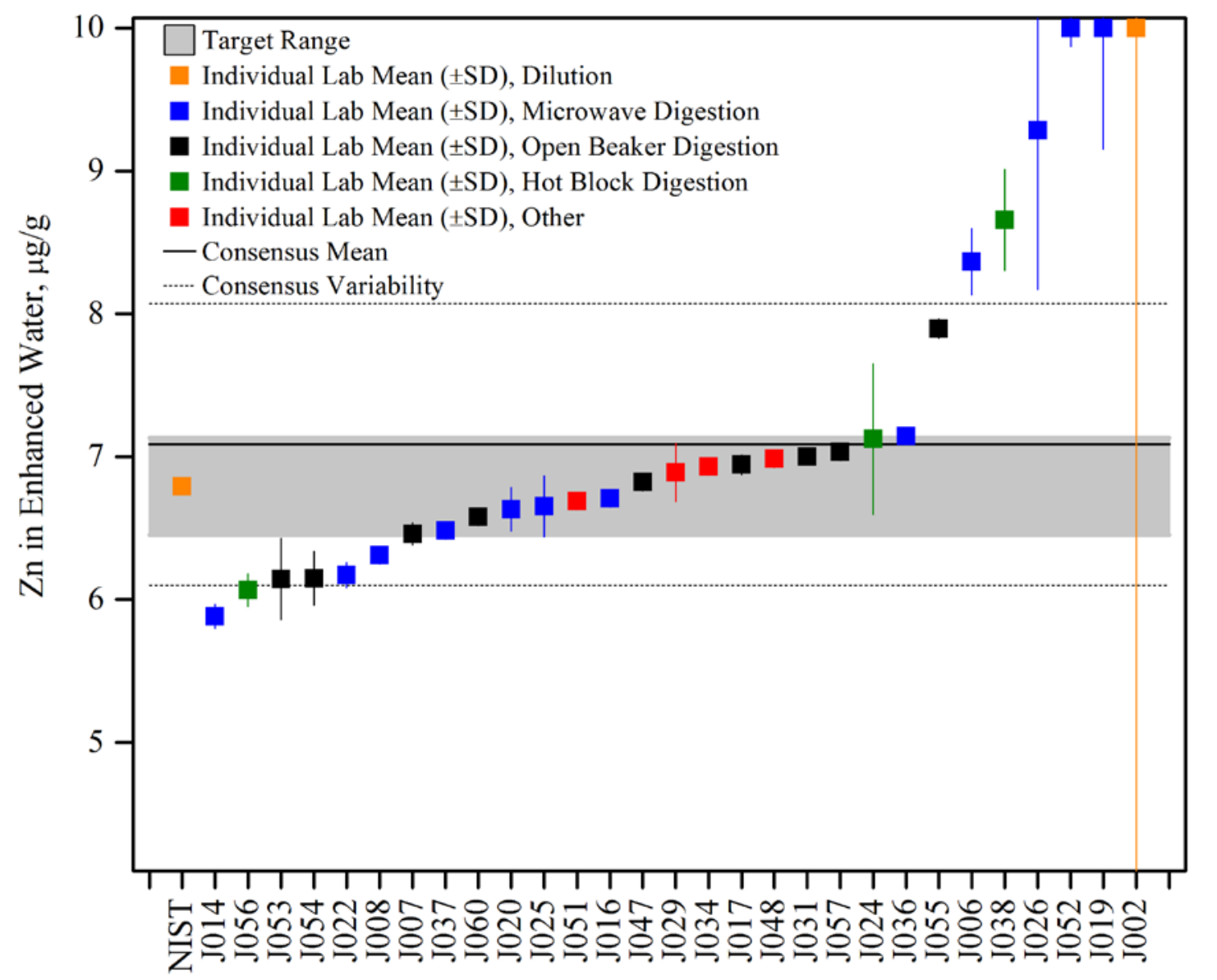

Figure 6. Zinc in enhanced water (data summary view - digestion method). In this view, individual laboratory data are plotted with the individual laboratory standard deviation (error bars). The color of the data point represents the sample preparation (digestion) procedure employed. The black solid line represents the consensus mean, and the black dotted lines represent the consensus variability calculated as one standard deviation about the consensus mean. The gray shaded region represents the target zone for "acceptable" performance, which encompasses the NIST value determined in triplicate by ICP-OES, bounded by an estimated relative uncertainty of $5 \%$. 


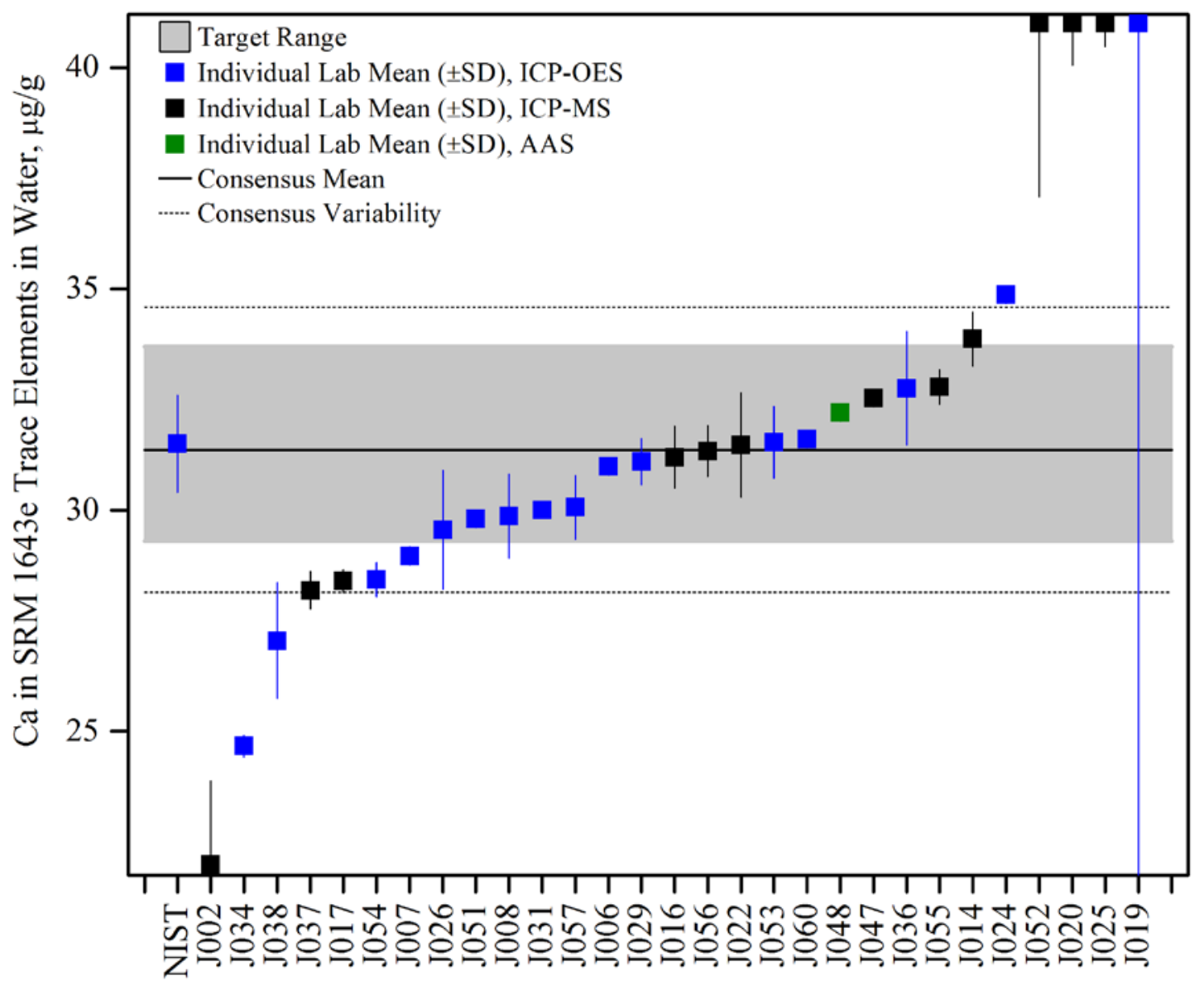

Figure 7. Calcium in SRM 1643e Trace Elements in Water (data summary view - instrumental method). In this view, individual laboratory data are plotted with the individual laboratory standard deviation (error bars). The color of the data point represents the instrumental method employed. The black solid line represents the consensus mean, and the black dotted lines represent the consensus variability calculated as one standard deviation about the consensus mean. The gray shaded region represents the target zone for "acceptable" performance, which encompasses the NIST certified value bounded by twice its uncertainty ( $\left.U_{95}\right)$. 


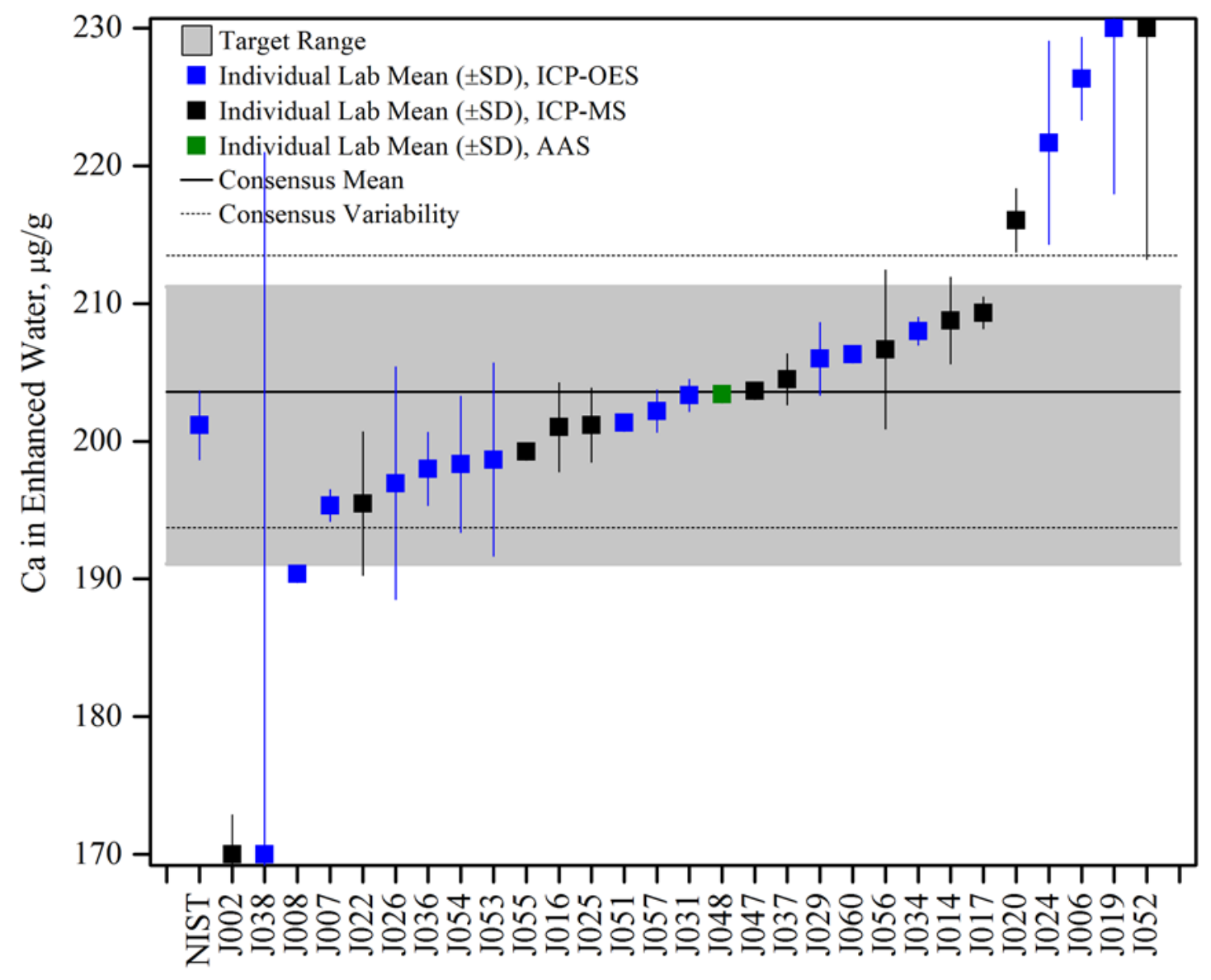

Figure 8. Calcium in enhanced water (data summary view - instrumental method). In this view, individual laboratory data are plotted with the individual laboratory standard deviation (error bars). The color of the data point represents the instrumental method employed. The black solid line represents the consensus mean, and the black dotted lines represent the consensus variability calculated as one standard deviation about the consensus mean. The gray shaded region represents the target zone for "acceptable" performance, which encompasses the NIST value determined in triplicate by ICP-OES, bounded by an estimated relative uncertainty of $5 \%$. 


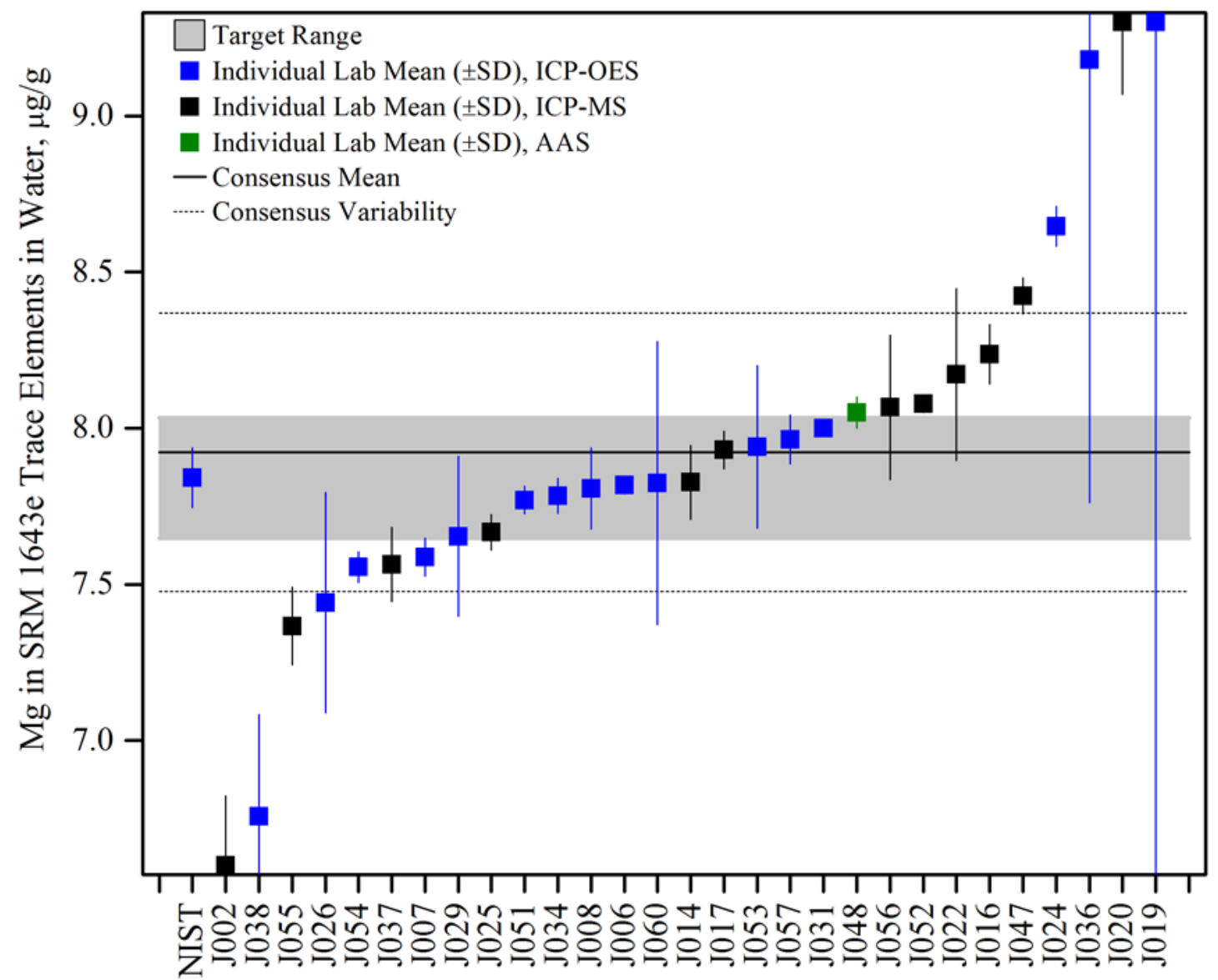

Figure 9. Magnesium in SRM 1643e Trace Elements in Water (data summary view instrumental method). In this view, individual laboratory data are plotted with the individual laboratory standard deviation (error bars). The color of the data point represents the instrumental method employed. The black solid line represents the consensus mean, and the black dotted lines represent the consensus variability calculated as one standard deviation about the consensus mean. The gray shaded region represents the target zone for "acceptable” performance, which encompasses the NIST certified value bounded by twice its uncertainty ( $\left.U_{95}\right)$. 


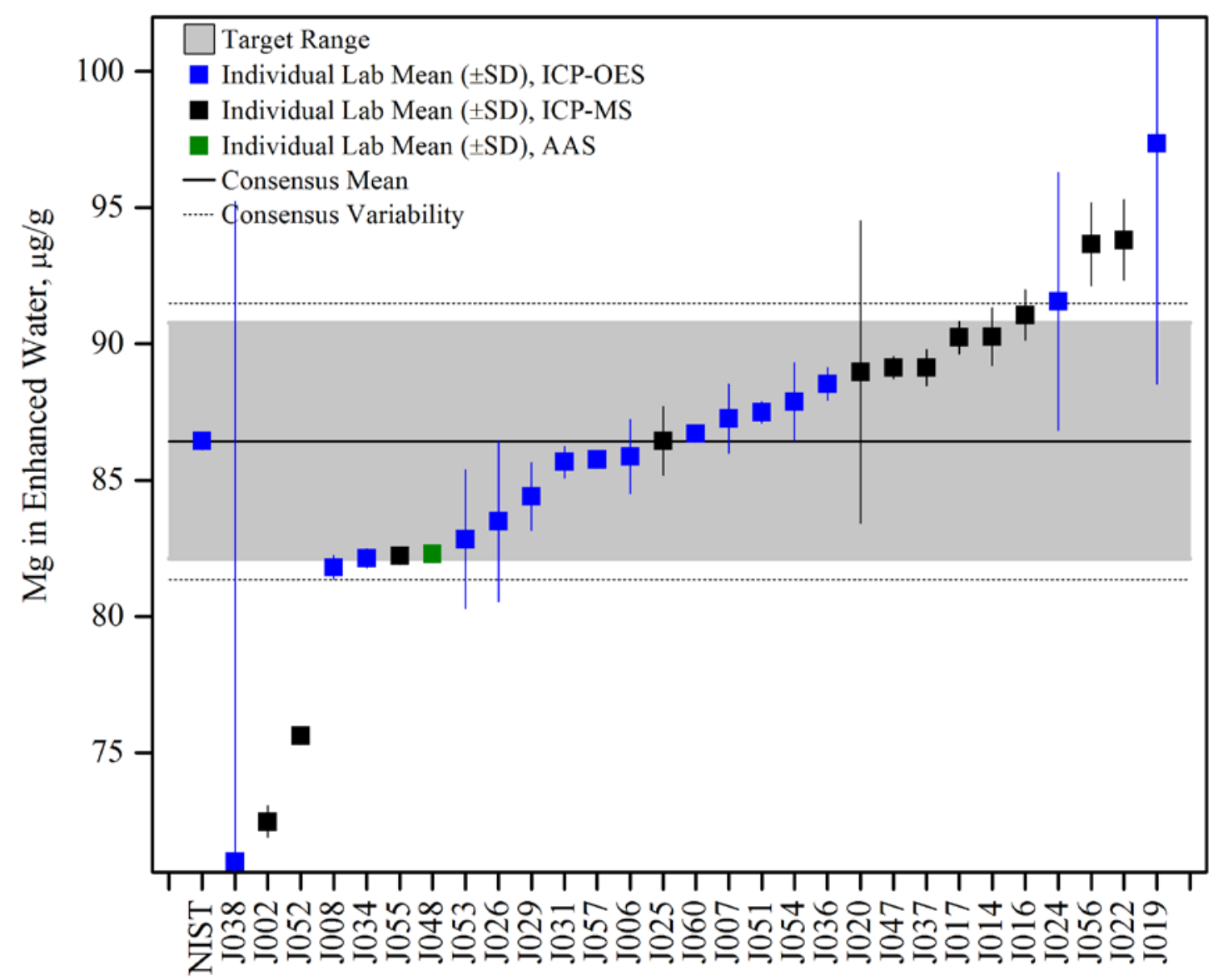

Figure 10. Magnesium in enhanced water (data summary view - instrumental method). In this view, individual laboratory data are plotted with the individual laboratory standard deviation (error bars). The color of the data point represents the instrumental method employed. The black solid line represents the consensus mean, and the black dotted lines represent the consensus variability calculated as one standard deviation about the consensus mean. The gray shaded region represents the target zone for "acceptable” performance, which encompasses the NIST value determined in triplicate by ICP-OES, bounded by an estimated relative uncertainty of $5 \%$. 


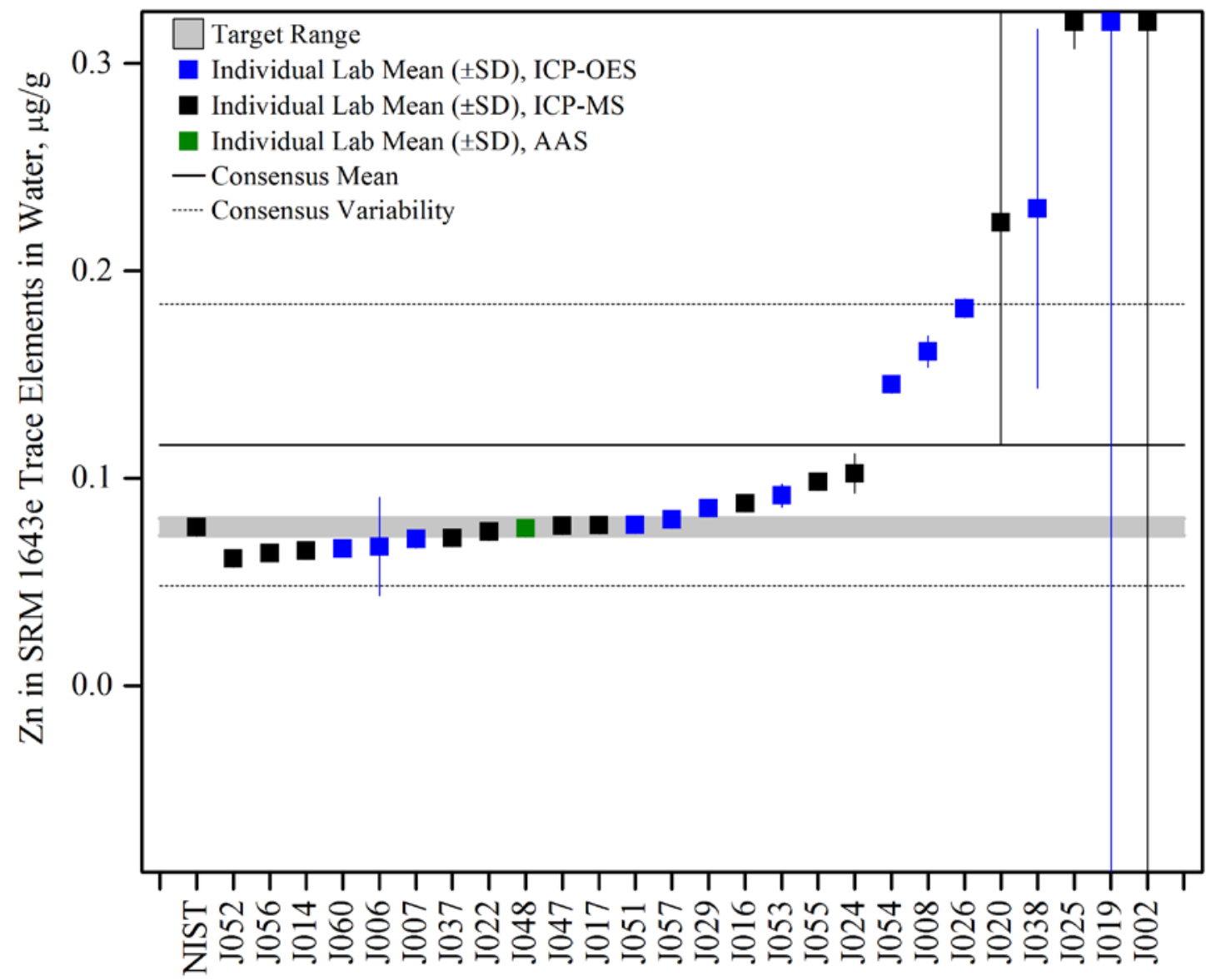

Figure 11. Zinc in SRM 1643e Trace Elements in Water (data summary view - instrumental method). In this view, individual laboratory data are plotted with the individual laboratory standard deviation (error bars). The color of the data point represents the instrumental method employed. The black solid line represents the consensus mean, and the black dotted lines represent the consensus variability calculated as one standard deviation about the consensus mean. The gray shaded region represents the target zone for "acceptable" performance, which encompasses the NIST certified value bounded by twice its uncertainty $\left(U_{95}\right)$. 


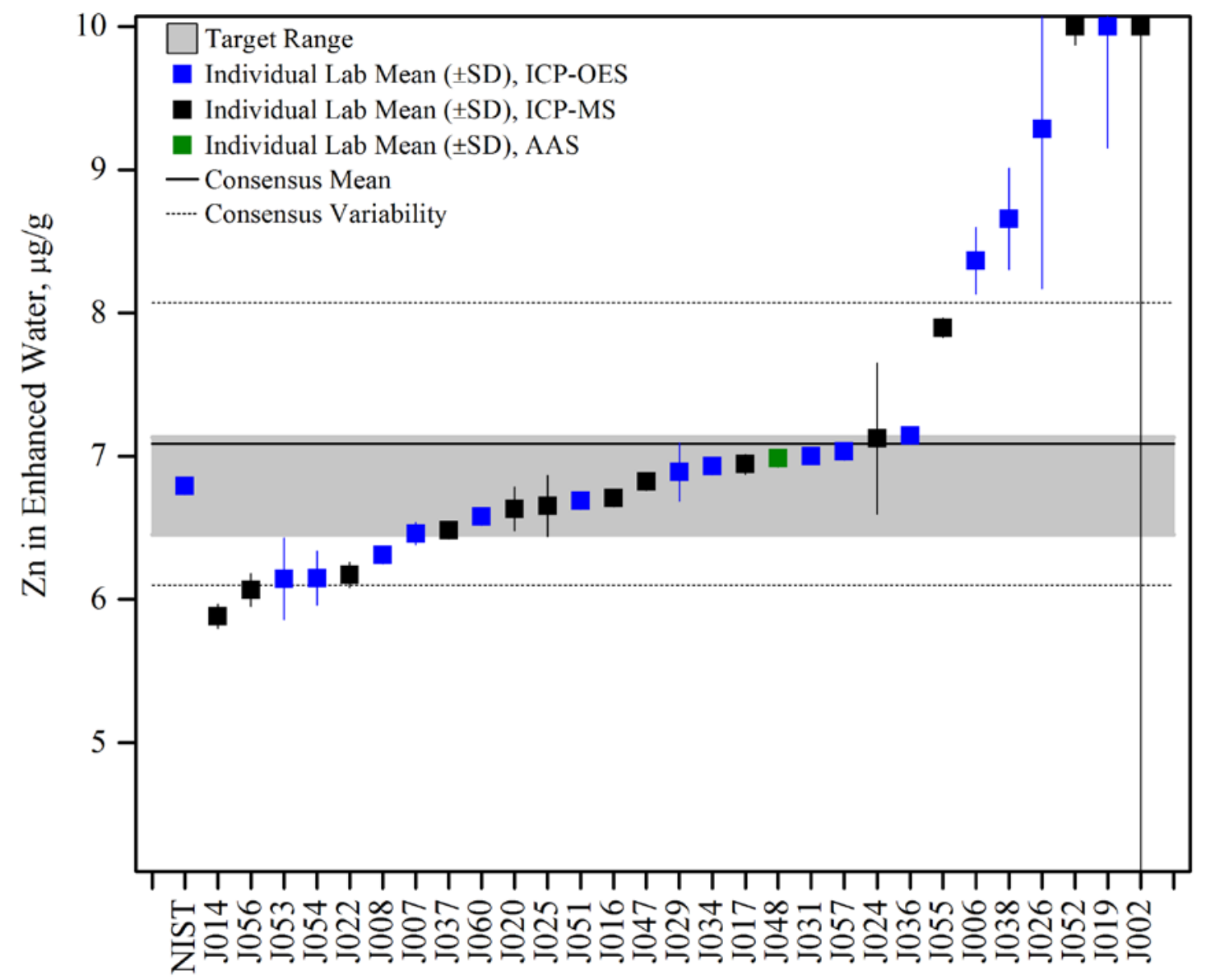

Figure 12. Zinc in enhanced water (data summary view - instrumental method). In this view, individual laboratory data are plotted with the individual laboratory standard deviation (error bars). The color of the data point represents the instrumental method employed. The black solid line represents the consensus mean, and the black dotted lines represent the consensus variability calculated as one standard deviation about the consensus mean. The gray shaded region represents the target zone for "acceptable" performance, which encompasses the NIST value determined in triplicate by ICP-OES, bounded by an estimated relative uncertainty of $5 \%$. 


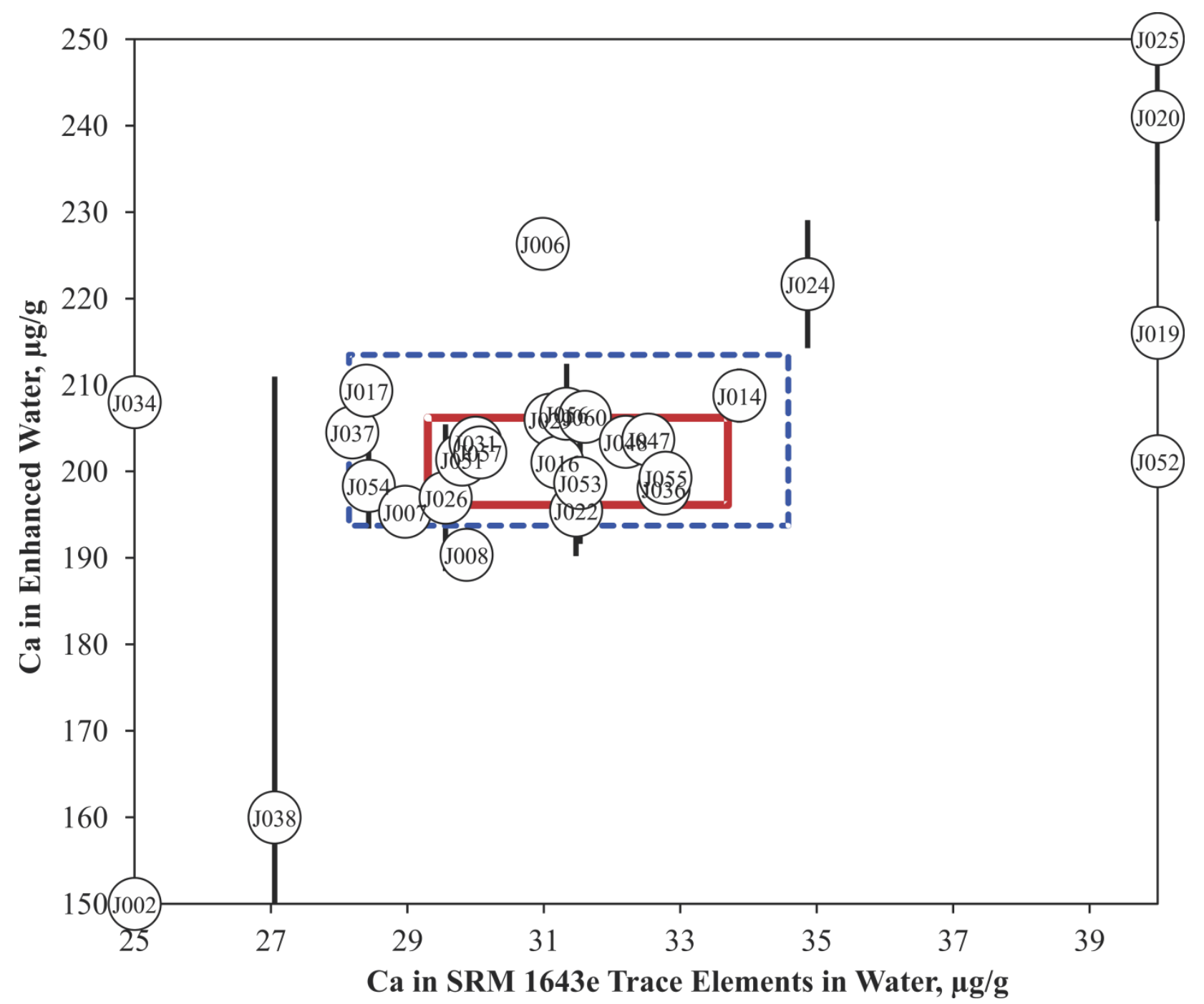

Figure 13. Calcium in SRM 1643e Trace Elements in Water and enhanced water (sample/control comparison view). In this view, the individual laboratory results for the control (SRM 1643e Trace Elements in Water) with a certified value for the analyte are compared to the results for an unknown (enhanced water). The solid red box represents the target zone for the control (x-axis) and unknown (y-axis). The dotted blue box represents the consensus zone for the control (x-axis) and the unknown sample (y-axis). 


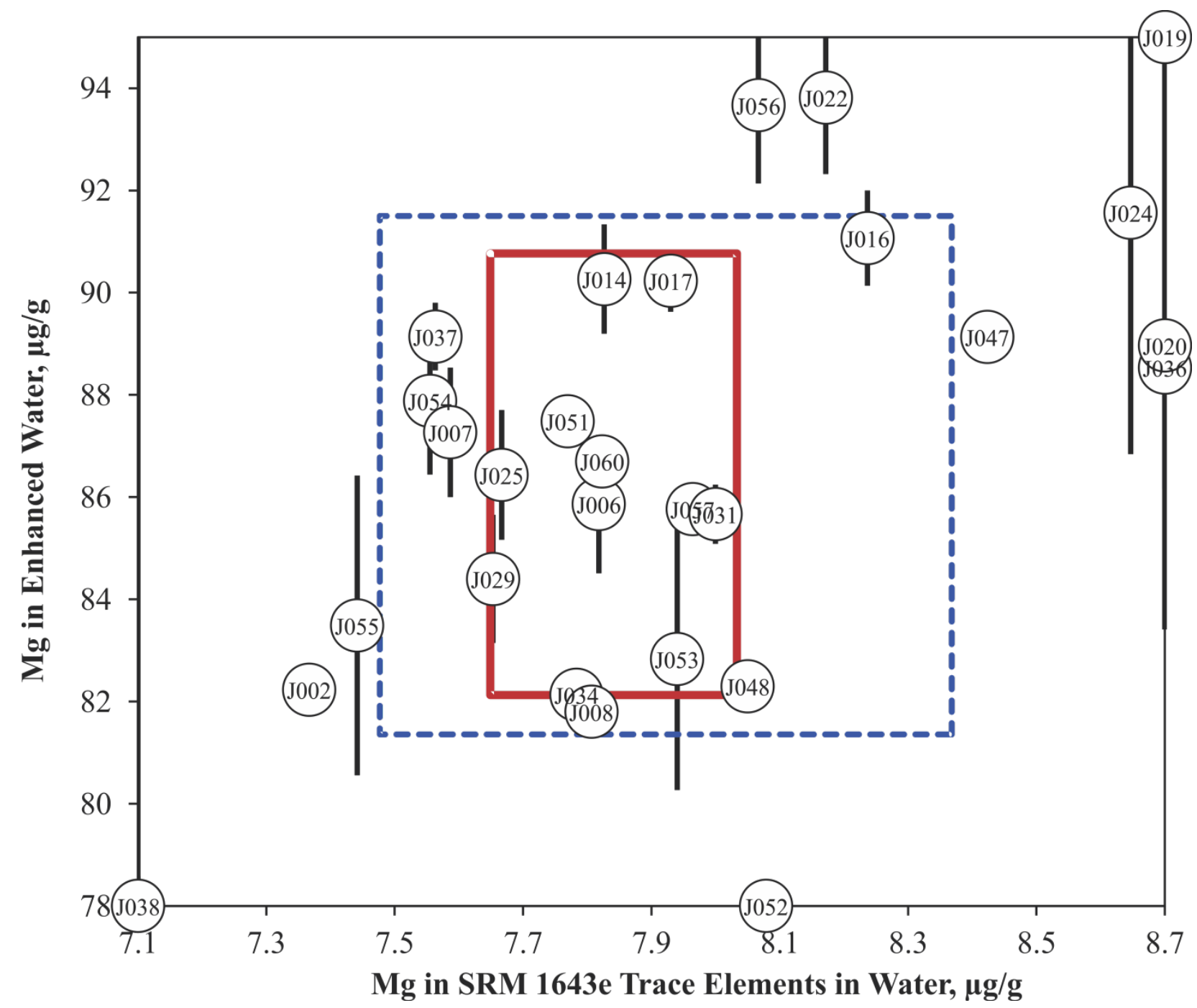

Figure 14. Magnesium in SRM 1643e Trace Elements in Water and enhanced water (sample/control comparison view). In this view, the individual laboratory results for the control (SRM 1643e Trace Elements in Water) with a certified value for the analyte are compared to the results for an unknown (enhanced water). The solid red box represents the target zone for the control (x-axis) and unknown (y-axis). The dotted blue box represents the consensus zone for the control (x-axis) and the unknown sample (y-axis). 


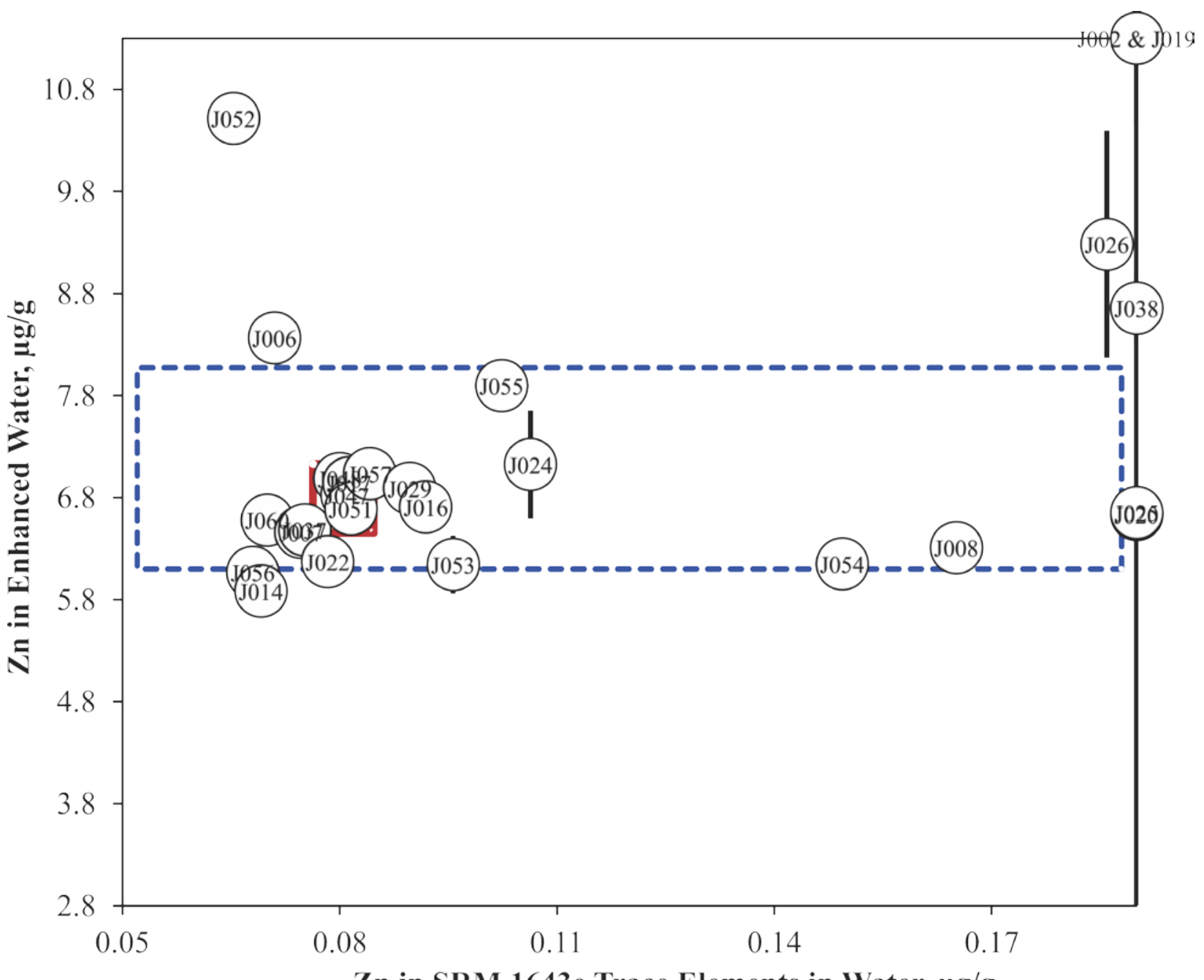

$\mathrm{Zn}$ in SRM 1643e Trace Elements in Water, $\mu \mathrm{g} / \mathrm{g}$

Figure 15. Zinc in SRM 1643e Trace Elements in Water and enhanced water (sample/control comparison view). In this view, the individual laboratory results for the control (SRM 1643e Trace Elements in Water) with a certified value for the analyte are compared to the results for an unknown (enhanced water). The solid red box represents the target zone for the control (x-axis) and unknown (y-axis). The dotted blue box represents the consensus zone for the control ( $\mathrm{x}$ axis) and the unknown sample (y-axis). 


\section{TOXIC ELEMENTS (As) IN ST. JOHN'S WORT DIETARY SUPPLEMENTS}

\section{Study Overview}

In this study, participants were provided with two NIST candidate SRMs, SRM 3262 Hypericum perforatum L. (St. John’s Wort) Aerial Parts and SRM 3264 Hypericum perforatum L. (St. John's Wort) Methanol Extract. Participants were asked to use in-house analytical methods to determine the mass fractions of arsenic (As) in each of the matrices and report values on an asreceived basis.

\section{Sample Information}

St. John's Wort Aerial Parts. Participants were provided with three packets, each containing approximately $3 \mathrm{~g}$ of St. John's Wort aerial parts. The plant was ground, homogenized, and heat-sealed inside 4 mil polyethylene bags, which were then sealed inside nitrogen-flushed aluminized plastic bags along with two packets of silica gel. Before use, participants were instructed to thoroughly mix the contents of each packet and use a sample size of at least $1.0 \mathrm{~g}$. Participants were asked to store the material at controlled room temperature, $10{ }^{\circ} \mathrm{C}$ to $30{ }^{\circ} \mathrm{C}$, and to prepare one sample and report one value from the each of the packets provided. Approximate analyte levels were not reported to participants prior to the study. The target value for arsenic in SRM 3262, $(145 \pm 8) \mathrm{ng} / \mathrm{g}$, was determined at NIST using ICP-MS and instrumental neutron activation analysis (INAA).

St. John's Wort Methanol Extract. Participants were provided with three packets, each containing approximately $1.6 \mathrm{~g}$ of a methanol extract of St. John's Wort. The extract was ground, homogenized, and heat-sealed inside 4 mil polyethylene bags, which were then sealed inside nitrogen-flushed aluminized plastic bags along with two packets of silica gel. Before use, participants were instructed to thoroughly mix the contents of each packet and use a sample size of at least $1.0 \mathrm{~g}$. Participants were asked to store the material at controlled room temperature, $10{ }^{\circ} \mathrm{C}$ to $30^{\circ} \mathrm{C}$, and to prepare one sample and report one value from the each of the packets provided. Approximate analyte levels were not reported to participants prior to the study. The target value for arsenic in SRM 3264, (34.8 \pm 3.0) ng/g, was determined at NIST using ICP-MS.

\section{Study Results}

- Thirty-four laboratories enrolled in this exercise and received samples. Twenty-seven laboratories reported results for arsenic in St. John's Wort aerial parts (79 \% participation). Twenty-six laboratories reported results for arsenic in St. John's Wort methanol extract (76 \% participation).

- The consensus means for arsenic were within the target range with high variability (24\% and $34 \%$ relative standard deviation (RSD) for the aerial parts and methanol extract, respectively).

- The greater number of low values reported for the aerial parts, compared to the extract, is most likely a result of incomplete digestions.

- The extremely high values reported for the extract material is most likely a result of incorrect calibration curves or dilutions of samples.

- A majority of the laboratories reported using microwave digestion (63\%) for sample preparation. Open beaker digestion (26\%) and hot block digestion (7\%) were also reported as methods of sample preparation.

- All of the laboratories reported using ICP-MS as their analytical method for analysis. 
Technical Recommendations

The following recommendations are based on results obtained by the participants in this study.

- While twice as many laboratories reported using microwave digestion for sample preparation than other methods reported, results did not vary based on the sample preparation method used.

- Arsenic is volatile, so care must be taken to not lose arsenic during sample preparation.

- With a vigorous microwave digestion (high heat) organoarsenic should all be converted to $\mathrm{As}^{+5}$ and any subsequent heating will not result in loss of As.

- Plant materials can be difficult to digest without the use of HF.

- Arsenic in the methanol extract is at a very low level and may be close to detection limits.

o Use a good calibration curve with low concentrations to help with accuracy.

o Use a sufficient number of blanks so an accurate method detection limit and limit of quantitation can be determined.

- Run a quality control sample of known concentration to ensure your method is performing as expected.

- Double-check all calculations for any errors. 
Table 5. Individual data summary table (NIST) for arsenic in St. John’s Wort dietary supplements.

\section{National Institute of Standards \& Technology}

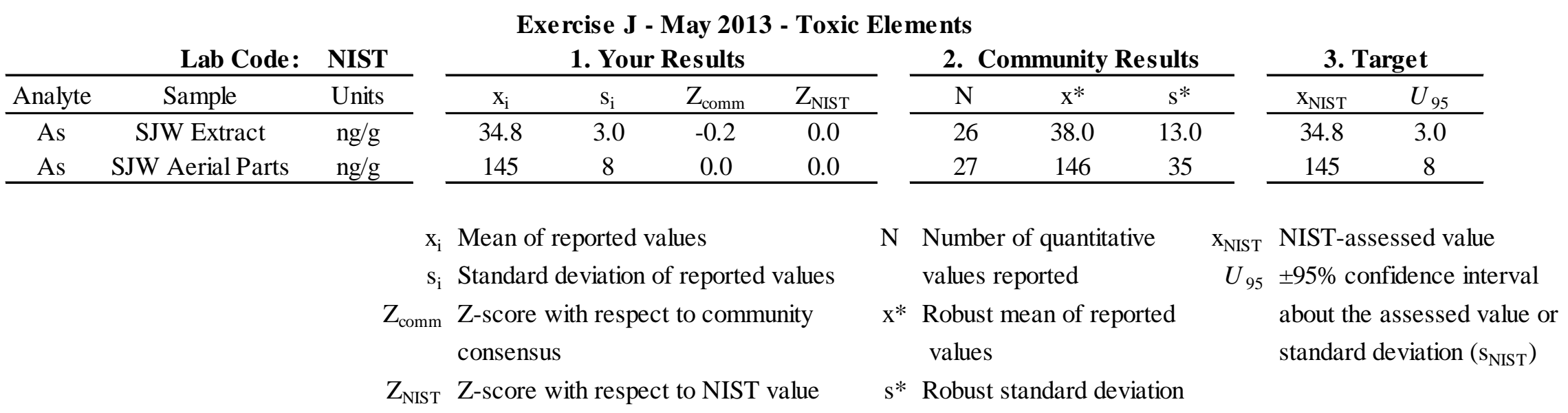


Table 6. Data summary table for arsenic in St. John’s Wort dietary supplements.

\begin{tabular}{|c|c|c|c|c|c|c|c|c|c|c|c|}
\hline & \multirow[b]{3}{*}{ Lab } & \multicolumn{10}{|c|}{ Arsenic } \\
\hline & & \multicolumn{5}{|c|}{ SRM 3264 St. John's Wort Extract (ng/g) } & \multicolumn{5}{|c|}{ SRM 3262 St. John's Wort Aerial Parts (ng/g) } \\
\hline & & A & B & C & Avg & SD & A & B & C & Avg & SD \\
\hline \multirow{35}{*}{ 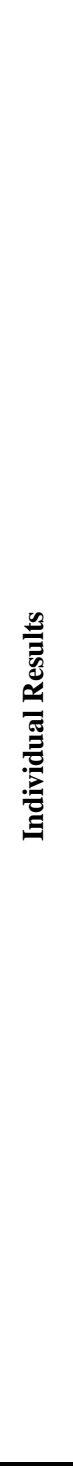 } & NIST & & & & 34.8 & 3.0 & & & & 145 & 8 \\
\hline & J002 & 29.9 & 23.5 & 30.3 & 27.9 & 3.8 & 123 & 128 & 118 & 123 & 5 \\
\hline & J006 & 27.4 & 43.5 & 26.4 & 32.4 & 9.6 & 140 & 153 & 154 & 149 & 8 \\
\hline & J007 & 42.0 & 38.8 & 46.8 & 42.5 & 4.0 & 136 & 122 & 116 & 125 & 10 \\
\hline & J008 & 76.5 & 73.0 & 66.8 & 72.1 & 4.9 & 197 & 216 & 214 & 209 & 10 \\
\hline & \multicolumn{11}{|l|}{ J009 } \\
\hline & J010 & 19.0 & 20.0 & 18.0 & 19.0 & 1.0 & 91 & 96 & 101 & 96 & 5 \\
\hline & J012 & 32.0 & 33.4 & 40.3 & 35.2 & 4.4 & 131 & 133 & 165 & 143 & 19 \\
\hline & J013 & 34.2 & 34.3 & 44.8 & 37.8 & 6.1 & 138 & 150 & 162 & 150 & 12 \\
\hline & J014 & 23.2 & 19.6 & 20.6 & 21.1 & 1.9 & 132 & 133 & 144 & 136 & 7 \\
\hline & J017 & & & & & & 144 & 154 & 147 & 148 & 5 \\
\hline & \multicolumn{11}{|l|}{ J018 } \\
\hline & \multicolumn{11}{|l|}{ J019 } \\
\hline & J020 & 39.6 & 38.5 & 37.3 & 38.5 & 1.2 & 143 & 128 & 128 & 133 & 9 \\
\hline & \multicolumn{11}{|l|}{ J021 } \\
\hline & J022 & 54.4 & 59.8 & 53.7 & 56.0 & 3.3 & 209 & 192 & 213 & 205 & 11 \\
\hline & J024 & 46.4 & 30.3 & 48.5 & 41.7 & 10.0 & 130 & 130 & 143 & 134 & 8 \\
\hline & J025 & 19.6 & 22.4 & 14.6 & 18.9 & 4.0 & 95 & 91 & 114 & 100 & 12 \\
\hline & J026 & & 35.5 & 32.6 & 34.1 & 2.0 & 176 & 190 & 183 & 183 & 7 \\
\hline & J029 & 46.2 & 50.8 & 51.6 & 49.5 & 2.9 & 168 & 135 & 134 & 146 & 19 \\
\hline & J030 & 40.0 & 40.0 & 40.0 & 40.0 & 0.0 & 170 & 190 & 170 & 177 & 12 \\
\hline & J031 & 114.0 & 117.0 & 121.0 & 117.3 & 3.5 & 124 & 116 & 113 & 118 & 6 \\
\hline & J033 & 37.3 & 38.8 & 38.8 & 38.3 & 0.9 & 165 & 169 & 174 & 169 & 5 \\
\hline & \multicolumn{11}{|l|}{ J035 } \\
\hline & J036 & 34.0 & 33.0 & 34.0 & 33.7 & 0.6 & 130 & 129 & 132 & 130 & 2 \\
\hline & J037 & 34.0 & 46.0 & 32.0 & 37.3 & 7.6 & 109 & 131 & 120 & 120 & 11 \\
\hline & \multicolumn{11}{|l|}{ J038 } \\
\hline & \multicolumn{11}{|l|}{ J041 } \\
\hline & J046 & 42.6 & 46.1 & 44.8 & 44.5 & 1.8 & 201 & 190 & 181 & 191 & 10 \\
\hline & J047 & 31.0 & 33.0 & 31.0 & 31.7 & 1.2 & 122 & 132 & 135 & 130 & 7 \\
\hline & J052 & 32.5 & 38.5 & 22.0 & 31.0 & 8.4 & 115 & 120 & 113 & 116 & 4 \\
\hline & J053 & 23.0 & 25.0 & 22.0 & 23.3 & 1.5 & 121 & 124 & 119 & 121 & 3 \\
\hline & J055 & 34.7 & 35.5 & 35.1 & 35.1 & 0.4 & 120 & 119 & 112 & 117 & 5 \\
\hline & J056 & 62.0 & 68.0 & 60.0 & 63.3 & 4.2 & 180 & 170 & 150 & 167 & 15 \\
\hline & J057 & 149.0 & 153.0 & 149.0 & 150.3 & 2.3 & 240 & 218 & 211 & 223 & 15 \\
\hline \multirow{5}{*}{ 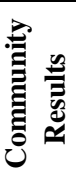 } & & \multirow{5}{*}{\multicolumn{3}{|c|}{$\begin{array}{l}\text { Consensus Mean } \\
\text { Consensus Standard Deviation } \\
\text { Maximum } \\
\text { Minimum } \\
\text { N }\end{array}$}} & \multirow{5}{*}{\multicolumn{2}{|c|}{$\begin{array}{c}38.0 \\
13.0 \\
150.3 \\
18.9 \\
26 \\
\end{array}$}} & \multirow{5}{*}{\multicolumn{3}{|c|}{$\begin{array}{l}\text { Consensus Mean } \\
\text { Consensus Standard Deviation } \\
\text { Maximum } \\
\text { Minimum } \\
\mathrm{N} \\
\end{array}$}} & 146 & \\
\hline & & & & & & & & & & 35 & \\
\hline & & & & & & & & & & 223 & \\
\hline & & & & & & & & & & 96 & \\
\hline & & & & & & & & & & 27 & \\
\hline
\end{tabular}




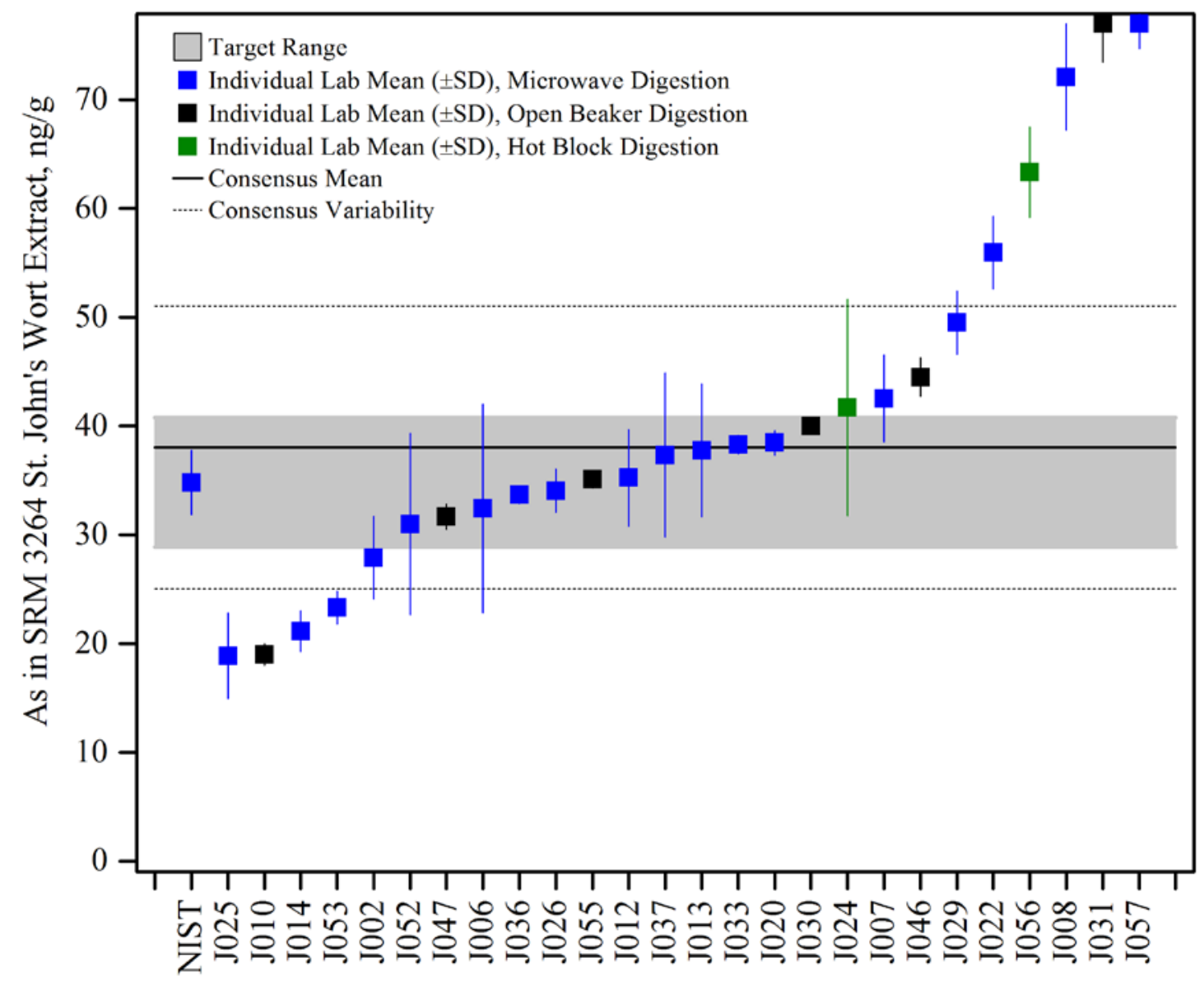

Figure 16. Arsenic in candidate SRM 3264 Hypericum perforatum L. (St. John's Wort) Methanol Extract (data summary view - digestion method). In this view, individual laboratory data are plotted with the individual laboratory standard deviation (error bars). The data are identified by digestion method in this graph. The black solid line represents the consensus mean, and the black dotted lines represent the consensus variability calculated as one standard deviation about the consensus mean. The gray shaded region represents the target zone for "acceptable" performance, which encompasses the NIST value determined by ICP-MS, bounded by an uncertainty estimated as twice the standard deviation. 


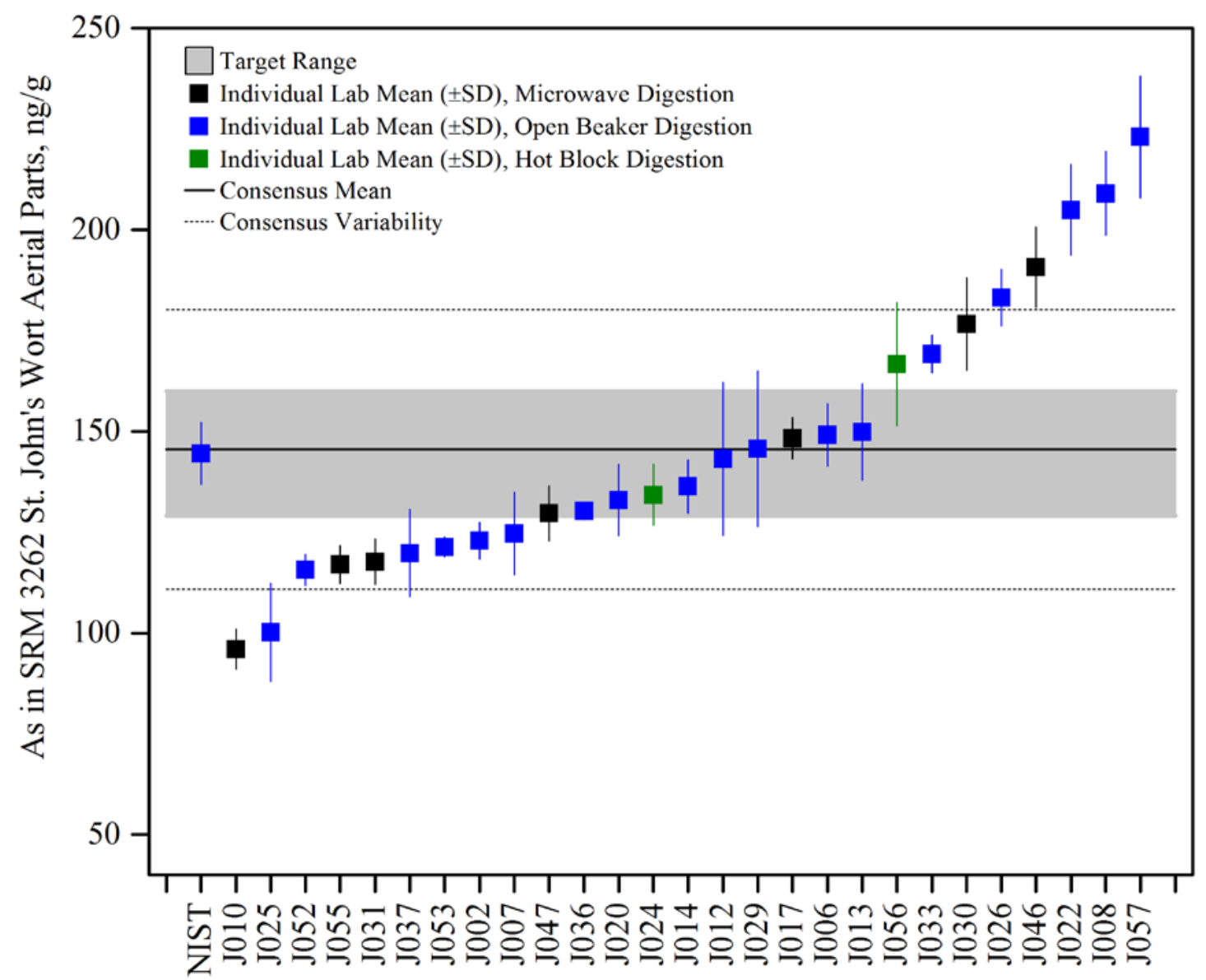

Figure 17. Arsenic in candidate SRM 3262 Hypericum perforatum L. (St. John's Wort) Aerial Parts (data summary view - digestion method). In this view, individual laboratory data are plotted with the individual laboratory standard deviation (error bars). The color of the data point represents the sample preparation (digestion) procedure employed. The black solid line represents the consensus mean, and the black dotted lines represent the consensus variability calculated as one standard deviation about the consensus mean. The gray shaded region represents the target zone for "acceptable" performance, which encompasses the mean of NIST value determined by ICP-MS and INAA, bounded by an uncertainty estimated as twice the combined standard deviation 


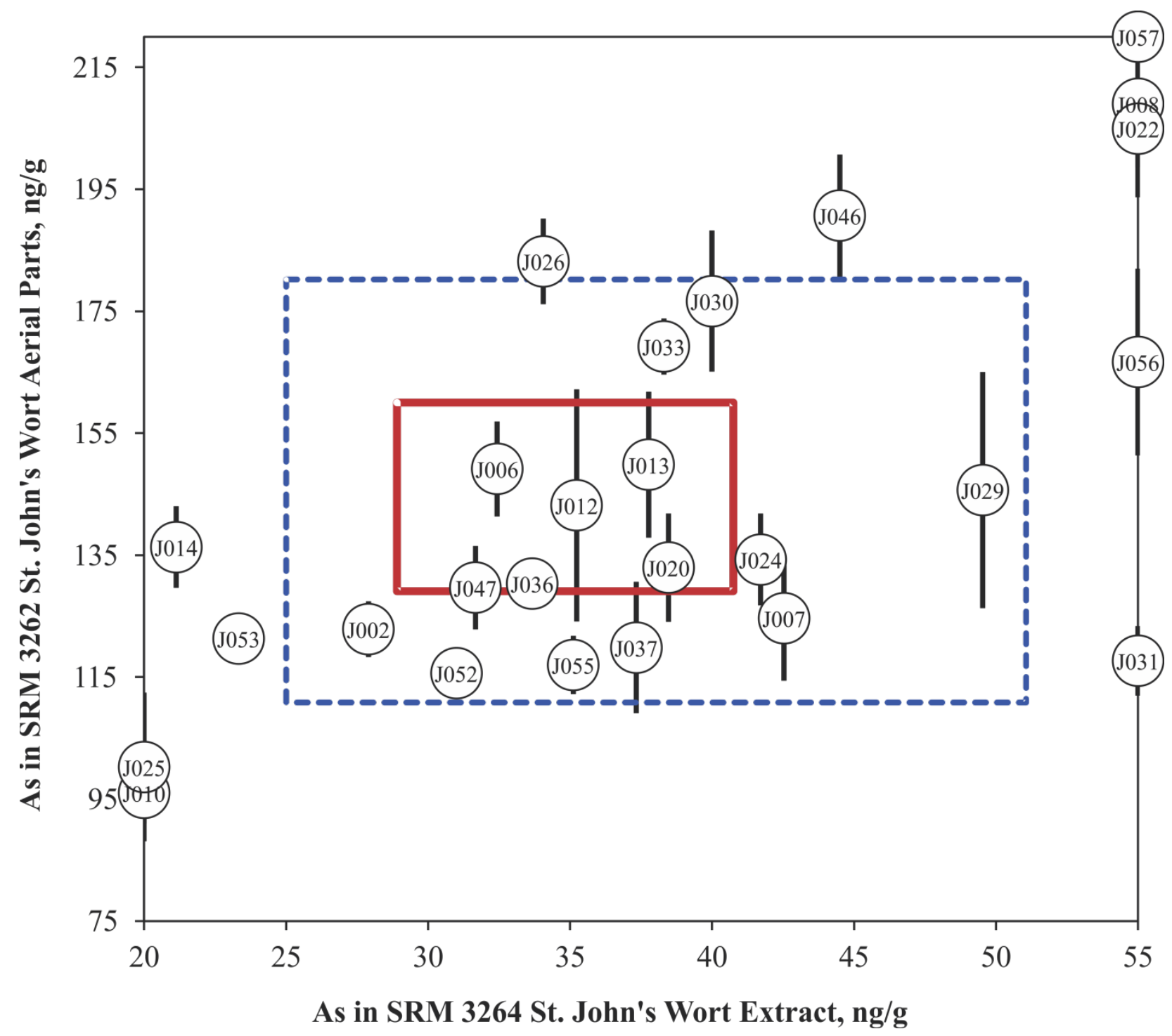

Figure 18. Arsenic in candidate SRM 3262 Hypericum perforatum L. (St. John's Wort) Aerial Parts and candidate SRM 3264 Hypericum perforatum L. (St. John's Wort) Methanol Extract (sample/sample comparison view). In this view, the individual laboratory results for one sample (St. John's Wort aerial parts) are compared to the results for a second sample (St. John's Wort extract). The solid red box represents the target zone for the two samples, St. John's Wort extract (x-axis) and St. John's Wort aerial parts (y-axis). The dotted blue box represents the consensus zone for St. John's Wort extract (x-axis) and St. John's Wort aerial parts (y-axis). 


\section{WATER-SOLUBLE VITAMINS $\left(\mathrm{B}_{5}, \mathrm{~B}_{6}\right)$ IN DIETARY SUPPLEMENTS}

\section{Study Overview}

In this study, participants were provided with one NIST SRM, SRM 3280 Multivitamin/

Multielement Tablets, and one bottle of commercially available enhanced water. Participants were asked to use in-house analytical methods to determine the mass fractions of vitamins $\mathrm{B}_{5}$ and $\mathrm{B}_{6}$ in each of the matrices and report values on an as-received basis. Participants were asked to report the vitamin $\mathrm{B}_{5}$ and $\mathrm{B}_{6}$ content as pantothenic acid and pyridoxine hydrochloride, respectively.

\section{Sample Information}

Multivitamin/Multielement Tablets. Participants were provided with one bottle containing 30 multivitamin/multielement tablets. Before use, participants were instructed to grind all 30 tablets, mix the resulting powder thoroughly, and use a sample size of at least $0.25 \mathrm{~g}$. Participants were asked to store the material at controlled room temperature, $10^{\circ} \mathrm{C}$ to $30{ }^{\circ} \mathrm{C}$, prepare three samples, and report three values from the single bottle provided. Approximate analyte levels were not reported to participants prior to the study. The NIST certified values and uncertainties for vitamin $B_{5}$ in SRM 3280 were determined by isotope dilution liquid chromatography with mass spectrometric detection (ID-LC/MS) following solvent extraction, in combination with data from numerous collaborating laboratories. The NIST certified values and uncertainties for vitamin B $_{6}$ in SRM 3280 were determined by LC with absorbance detection (LC/abs) and ID-LC/MS following solvent extraction, in combination with data from numerous collaborating laboratories. The certified values are reported in the table below, both on a drymass basis and after correction for moisture of the material (1.37 \%).

$\begin{array}{ccc}c & \begin{array}{c}\text { Certified Mass Fraction } \\ \text { in SRM 3280 (mg/g) } \\ \text { Analyte }\end{array} & \begin{array}{c}\text { Certified Mass Fraction } \\ \text { in SRM } 3280(\mathrm{mg} / \mathrm{g})\end{array} \\ \begin{array}{c}\text { (dry-mass basis) } \\ \text { Pantothenic Acid }\left(\mathrm{B}_{5}\right)\end{array} & \begin{array}{c}7.30 \pm 0.96 \\ \text { (as-received basis) }\end{array} \\ \text { Pyridoxine Hydrochloride }\left(\mathrm{B}_{6}\right) & 1.81 \pm 0.17 & 7.20 \pm 0.95\end{array}$

Enhanced Water. Participants were provided with one $600 \mathrm{~mL}$ bottle of commercially available enhanced water. Before use, participants were instructed to thoroughly mix the contents of the bottle, and a sample size of at least $1.0 \mathrm{~mL}$ was recommended. Participants were asked to store the material at controlled room temperature, $10^{\circ} \mathrm{C}$ to $30^{\circ} \mathrm{C}$, prepare three samples, and report three values from the single bottle provided. Approximate analyte levels were not reported to participants prior to the study. Certified values are not available for this material; NIST provided values for vitamins $B_{5}$ and $B_{6}$ based on triplicate analysis using ID-LC/MS. The NIST values in enhanced water are reported in the table below with an estimated uncertainty of $5 \%$.

\author{
Analyte \\ Pantothenic Acid \\ Pyridoxine Hydrochloride
}

Estimated Mass Fraction in Commercial Enhanced Water ( $\mu \mathrm{g} / \mathrm{g}$ )

$$
\begin{aligned}
39.3 & \pm 0.1 \\
6.53 & \pm 0.02
\end{aligned}
$$




\section{$\underline{\text { Study Results }}$}

- Thirty-three laboratories enrolled in this exercise and received samples. Twenty-three laboratories reported results for vitamin $B_{5}$ in SRM 3280 (70\% participation) and 25 laboratories reported results for vitamin $\mathrm{B}_{6}$ in SRM 3280 (76\% participation). Twentytwo laboratories reported results for both vitamins $B_{5}$ and $B_{6}$ in enhanced water $(67 \%$ participation).

- The consensus mean was within the target range for vitamins $B_{5}$ and $B_{6}$ in SRM 3280 and for vitamin $\mathrm{B}_{6}$ in the enhanced water. The variability in these measurements was excellent, with $10 \%$ RSD for both vitamins in the multivitamin sample and $17 \%$ RSD for vitamin $B_{6}$ in the enhanced water.

- The consensus mean for vitamin $\mathrm{B}_{5}$ in the enhanced water was slightly below the target range, and the variability was slightly higher at $23 \% \mathrm{RSD}$.

- A majority of the laboratories reported using solvent extraction (88\%) as the sample preparation method. Laboratories also reported using acid hydrolysis (8 \%).

- A majority of the laboratories reported using LC/Abs (88 \%) as their instrumental method for analysis. Microbiological assay (8 \%), LC/MS (4 \%), and Direct Analysis in Real Time Mass Spectrometry (DART-MS) (4\%) were also reported.

- The level of vitamin $B_{5}$ in enhanced water sample was 200 times lower than that in the multivitamin tablet, which may have resulted in analytical challenges. Laboratories using LC/Abs may have had difficulty detecting pantothenic acid accurately.

\section{Technical Recommendations}

The following recommendations are based on results obtained by the participants in this study.

- In general, the results for this study were very good, and no analytical method was identified as being exceptionally good or problematic.

- When using LC-absorbance for a molecule like pantothenic acid without a chromophore, care must be taken to remove matrix interferences. Spiking of the sample used during method development may also be helpful to be sure that small chromatographic peaks are correctly identified. 
Table 7. Individual data summary table (NIST) for water-soluble vitamins in dietary supplements.

\section{National Institute of Standards \& Technology}

\begin{tabular}{ccc} 
& Lab Code: & NIST \\
\hline Analyte & Sample & Units \\
\hline B5 & Multivitamin & $\mathrm{mg} / \mathrm{g}$ \\
B5 & Enhanced Water & $\mu \mathrm{g} / \mathrm{g}$ \\
\hline B6 & Multivitamin & $\mathrm{mg} / \mathrm{g}$ \\
B6 & Enhanced Water & $\mu \mathrm{g} / \mathrm{g}$ \\
\hline
\end{tabular}

\section{Exercise J - May 2013 - Water-Soluble Vitamins}

\begin{tabular}{cccc}
\multicolumn{4}{c}{ 1. Your Results } \\
\hline $\mathrm{x}_{\mathrm{i}}$ & $\mathrm{s}_{\mathrm{i}}$ & $\mathrm{Z}_{\text {comm }}$ & $\mathrm{Z}_{\text {NIST }}$ \\
\hline 7.20 & 0.95 & -0.5 & 0.0 \\
39.3 & 0.1 & 0.6 & 0.0 \\
\hline 1.79 & 0.17 & 0.3 & 0.0 \\
6.53 & 0.02 & -0.2 & 0.0 \\
\hline
\end{tabular}

\begin{tabular}{ccc}
\multicolumn{3}{c}{ 2. Community Results } \\
\hline $\mathrm{N}$ & $\mathrm{x}^{*}$ & $\mathrm{~s}^{*}$ \\
\hline 23 & 7.58 & 0.76 \\
22 & 34.5 & 8.0 \\
\hline 25 & 1.73 & 0.18 \\
22 & 6.72 & 1.17 \\
\hline
\end{tabular}

\begin{tabular}{cc}
\multicolumn{2}{c}{ 3. Target } \\
\hline $\mathrm{X}_{\mathrm{NIST}}$ & $U_{95}$ \\
\hline 7.20 & 0.95 \\
39.3 & 2.0 \\
\hline 1.79 & 0.17 \\
6.53 & 0.33 \\
\hline
\end{tabular}

$\mathrm{x}_{\mathrm{i}}$ Mean of reported values

$\mathrm{s}_{\mathrm{i}}$ Standard deviation of reported values

$\mathrm{Z}_{\text {comm }}$ Z-score with respect to community consensus

$\mathrm{Z}_{\mathrm{NIST}} \mathrm{Z}$-score with respect to NIST value
$\mathrm{N}$ Number of quantitative values reported

$\mathrm{x}^{*}$ Robust mean of reported values

s* Robust standard deviation $\mathrm{x}_{\text {NIST }}$ NIST-assessed value

$U_{95} \pm 95 \%$ confidence interval about the assessed value or standard deviation $\left(\mathrm{s}_{\mathrm{NIST}}\right)$ 
Table 8. Data summary table for vitamin $\mathrm{B}_{5}$ (pantothenic acid) in dietary supplements.

\begin{tabular}{|c|c|c|c|c|c|c|c|c|c|c|c|}
\hline & \multirow[b]{3}{*}{ Lab } & \multicolumn{10}{|c|}{ Pantothenic Acid } \\
\hline & & \multicolumn{5}{|c|}{ SRM 3280 Multivitamin/Multielement Tablets (mg/g) } & \multicolumn{5}{|c|}{ Enhanced Water ( $\mu \mathrm{g} / \mathrm{g})$} \\
\hline & & A & B & C & Avg & SD & $\mathbf{A}$ & B & $\mathbf{C}$ & Avg & SD \\
\hline \multirow{34}{*}{ 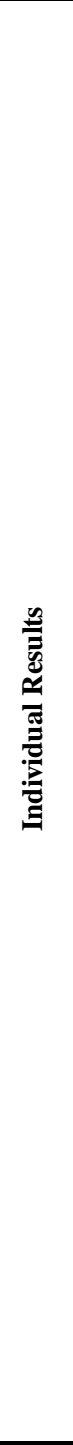 } & NIST & & & & 7.20 & 0.95 & 39.1 & 39.4 & 39.3 & 39.3 & 0.1 \\
\hline & J002 & & & & & & & & & & \\
\hline & J003 & & & & & & & & & & \\
\hline & J004 & & & & & & & & & & \\
\hline & J008 & 9.67 & 9.33 & 9.11 & 9.37 & 0.28 & 41.1 & 38.2 & 40.1 & 39.8 & 1.5 \\
\hline & J011 & 8.92 & 8.92 & 8.97 & 8.94 & 0.03 & 39.6 & 39.4 & 39.5 & 39.5 & 0.1 \\
\hline & J015 & 8.01 & 8.09 & 7.75 & 7.95 & 0.18 & 6.6 & 6.5 & 6.5 & 6.5 & 0.0 \\
\hline & J016 & & & & & & & & & & \\
\hline & J017 & 7.49 & 7.48 & 7.63 & 7.53 & 0.08 & 35.5 & 42.1 & 35.0 & 37.5 & 4.0 \\
\hline & J018 & 8.00 & 8.00 & 8.00 & 8.00 & 0.00 & 49.0 & 50.0 & 50.0 & 49.7 & 0.6 \\
\hline & J020 & 6.79 & 6.51 & 6.65 & 6.65 & 0.14 & 27.1 & 27.0 & 26.9 & 27.0 & 0.1 \\
\hline & J021 & 3.84 & 4.05 & 3.79 & 3.89 & 0.14 & 14.1 & 14.0 & 14.1 & 14.0 & 0.1 \\
\hline & J022 & 7.50 & 7.40 & 7.40 & 7.43 & 0.06 & 29.3 & 29.2 & 29.3 & 29.3 & 0.1 \\
\hline & J025 & 7.40 & 7.50 & 7.40 & 7.43 & 0.06 & 35.0 & 33.0 & 35.0 & 34.3 & 1.2 \\
\hline & J028 & & & & & & & & & & \\
\hline & J029 & 7.26 & 7.47 & 7.47 & 7.40 & 0.12 & 37.8 & 38.9 & 38.8 & 38.5 & 0.6 \\
\hline & J030 & 7.59 & 7.58 & 7.75 & 7.64 & 0.10 & 36.5 & 39.0 & 38.0 & 37.8 & 1.3 \\
\hline & J031 & 7.53 & 7.66 & 7.65 & 7.61 & 0.07 & 34.7 & 34.6 & 33.9 & 34.4 & 0.4 \\
\hline & J034 & 7.14 & 6.87 & 6.76 & 6.92 & 0.20 & 33.2 & 35.4 & 35.4 & 34.6 & 1.3 \\
\hline & J036 & 7.24 & 1.79 & 7.23 & 5.42 & 3.14 & 36.2 & 36.1 & 36.1 & 36.1 & 0.0 \\
\hline & J037 & & & & & & & & & & \\
\hline & J038 & 8.26 & 7.93 & 7.89 & 8.03 & 0.20 & & & & & \\
\hline & J039 & & & & & & & & & & \\
\hline & J042 & 7.95 & 7.90 & 7.34 & 7.73 & 0.34 & 30.9 & 35.6 & 36.9 & 34.5 & 3.2 \\
\hline & J043 & 8.23 & 7.99 & 8.12 & 8.12 & 0.12 & 43.0 & 42.4 & 46.6 & 44.0 & 2.3 \\
\hline & J044 & 7.30 & 7.20 & 7.19 & 7.23 & 0.06 & 32.9 & 31.1 & 36.2 & 33.4 & 2.6 \\
\hline & J045 & 6.80 & 6.80 & 6.80 & 6.80 & 0.00 & 34300.0 & 35200.0 & 34700.0 & 34733.3 & 450.9 \\
\hline & J047 & 7.13 & 7.13 & 7.30 & 7.19 & 0.10 & 34.9 & 36.0 & 35.8 & 35.6 & 0.6 \\
\hline & J052 & & & & & & & & & & \\
\hline & J053 & & & & & & & & & & \\
\hline & J054 & 11.40 & 11.20 & 11.70 & 11.43 & 0.25 & 20.1 & 20.3 & 20.1 & 20.2 & 0.1 \\
\hline & J057 & 8.45 & 7.99 & 7.95 & 8.13 & 0.28 & & & & & \\
\hline & J058 & 7.57 & 7.54 & 7.63 & 7.58 & 0.05 & 27.0 & 26.4 & 26.9 & 26.8 & 0.3 \\
\hline & J059 & & & & & & 35.2 & 34.2 & 35.0 & 34.8 & 0.6 \\
\hline \multirow{5}{*}{ 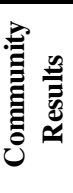 } & & \multicolumn{3}{|c|}{ Consensus Mean } & \multicolumn{2}{|l|}{7.58} & \multicolumn{3}{|c|}{ Consensus Mean } & 34.5 & \\
\hline & & \multicolumn{3}{|c|}{ Consensus Standard Deviation } & \multicolumn{2}{|l|}{0.76} & \multicolumn{3}{|c|}{ Consensus Standard Deviation } & 8.0 & \\
\hline & & \multicolumn{3}{|c|}{ Maximum } & \multicolumn{2}{|l|}{11.43} & \multicolumn{3}{|c|}{ Maximum } & 34733.3 & \\
\hline & & \multicolumn{3}{|l|}{ Minimum } & \multicolumn{2}{|l|}{3.89} & \multicolumn{3}{|l|}{ Minimum } & 6.5 & \\
\hline & & \multicolumn{3}{|l|}{$\mathrm{N}$} & \multicolumn{2}{|l|}{23} & \multicolumn{3}{|l|}{$\mathrm{N}$} & 22 & \\
\hline
\end{tabular}


Table 9. Data summary table for vitamin $\mathrm{B}_{6}$ (pyridoxine hydrochloride) in dietary supplements.

\begin{tabular}{|c|c|c|c|c|c|c|c|c|c|c|c|}
\hline & \multirow[b]{3}{*}{ Lab } & \multicolumn{10}{|c|}{ Pyridoxine Hydrochloride } \\
\hline & & \multicolumn{5}{|c|}{ SRM 3280 Multivitamin/Multielement Tablets (mg/g) } & \multicolumn{5}{|c|}{ Enhanced Water ( $\mu \mathrm{g} / \mathrm{g})$} \\
\hline & & A & B & C & Avg & SD & $\mathbf{A}$ & B & $\mathbf{C}$ & Avg & SD \\
\hline \multirow{34}{*}{ 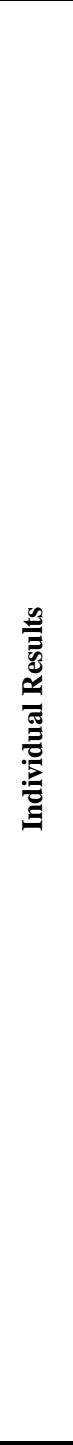 } & NIST & & & & 1.79 & 0.17 & 6.51 & 6.52 & 6.55 & 6.53 & 0.02 \\
\hline & J002 & & & & & & & & & & \\
\hline & J003 & & & & & & & & & & \\
\hline & J004 & & & & & & & & & & \\
\hline & J008 & 1.79 & 1.57 & 1.49 & 1.62 & 0.16 & 6.63 & 6.56 & 6.59 & 6.59 & 0.04 \\
\hline & J011 & 2.15 & 2.12 & 2.10 & 2.12 & 0.03 & 6.58 & 6.52 & 6.65 & 6.58 & 0.07 \\
\hline & J015 & 1.95 & 1.95 & 1.97 & 1.96 & 0.01 & 7.10 & 7.13 & 7.13 & 7.12 & 0.02 \\
\hline & J016 & & & & & & & & & & \\
\hline & J017 & 1.65 & 1.62 & 1.67 & 1.65 & 0.03 & 7.61 & 7.43 & 7.60 & 7.55 & 0.10 \\
\hline & J018 & 1.54 & 1.57 & 1.51 & 1.54 & 0.03 & 7.00 & 7.00 & 7.00 & 7.00 & 0.00 \\
\hline & J020 & 1.80 & 1.79 & 1.77 & 1.79 & 0.02 & 6.28 & 6.33 & 6.28 & 6.30 & 0.03 \\
\hline & J021 & 1.74 & 1.75 & 1.82 & 1.77 & 0.04 & 8.24 & 8.20 & 8.13 & 8.19 & 0.06 \\
\hline & J022 & 1.84 & 1.82 & 1.81 & 1.82 & 0.02 & 7.00 & 7.00 & 7.00 & 7.00 & 0.00 \\
\hline & J025 & 1.96 & 1.92 & 1.94 & 1.94 & 0.02 & 6.70 & 6.80 & 6.70 & 6.73 & 0.06 \\
\hline & J028 & & & & & & & & & & \\
\hline & J029 & 1.87 & 1.92 & 1.86 & 1.88 & 0.03 & 5.45 & 5.23 & 5.30 & 5.33 & 0.11 \\
\hline & J030 & 1.73 & 1.89 & 1.84 & 1.82 & 0.08 & 6.59 & 6.55 & 6.66 & 6.60 & 0.06 \\
\hline & J031 & 1.75 & 1.81 & 1.78 & 1.78 & 0.03 & 7.05 & 6.65 & 6.45 & 6.72 & 0.31 \\
\hline & J034 & 1.51 & 1.50 & 1.43 & 1.48 & 0.04 & 5.15 & 4.91 & 4.96 & 5.01 & 0.13 \\
\hline & J036 & 1.71 & 1.64 & 1.73 & 1.69 & 0.05 & 6.18 & 6.18 & 6.42 & 6.26 & 0.14 \\
\hline & J037 & & & & & & & & & & \\
\hline & J038 & 1.87 & 1.84 & 1.81 & 1.84 & 0.03 & & & & & \\
\hline & J039 & & & & & & & & & & \\
\hline & J042 & 1.83 & 1.81 & 1.81 & 1.82 & 0.01 & 5.57 & 5.55 & 5.55 & 5.56 & 0.01 \\
\hline & J042 & 1.70 & 1.69 & 1.72 & 1.70 & 0.02 & & & & & \\
\hline & J044 & 1.53 & 1.49 & 1.48 & 1.50 & 0.03 & 5.70 & 5.97 & 5.62 & 5.76 & 0.18 \\
\hline & J045 & 1.64 & 1.69 & 1.74 & 1.69 & 0.05 & 6800.80 & 6814.40 & 6814.80 & 6810.00 & 7.97 \\
\hline & J047 & 1.65 & 1.66 & 1.72 & 1.68 & 0.04 & 6.50 & 6.50 & 6.50 & 6.50 & 0.00 \\
\hline & J052 & 1.83 & 1.80 & & 1.82 & 0.02 & & & & & \\
\hline & J053 & 1.35 & 1.37 & 1.30 & 1.34 & 0.03 & 5.10 & 5.31 & & 5.21 & 0.15 \\
\hline & J054 & 1.27 & 1.25 & 1.29 & 1.27 & 0.02 & 31.24 & 32.12 & 30.13 & 31.16 & 1.00 \\
\hline & J057 & 1.71 & 1.67 & 1.67 & 1.68 & 0.02 & & & & & \\
\hline & J058 & 1.89 & 1.85 & 1.86 & 1.87 & 0.02 & 8.76 & 8.72 & 8.74 & 8.74 & 0.02 \\
\hline & J059 & & & & & & 6.56 & 6.19 & 6.48 & 6.41 & 0.19 \\
\hline \multirow{5}{*}{ 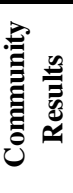 } & & \multicolumn{3}{|c|}{ Consensus Mean } & \multicolumn{2}{|l|}{1.73} & \multicolumn{3}{|c|}{ Consensus Mean } & 6.72 & \\
\hline & & \multicolumn{3}{|c|}{ Consensus Standard Deviation } & \multicolumn{2}{|l|}{0.18} & \multicolumn{3}{|c|}{ Consensus Standard Deviation } & 1.17 & \\
\hline & & \multicolumn{3}{|c|}{ Maximum } & \multicolumn{2}{|l|}{2.12} & \multicolumn{3}{|c|}{ Maximum } & 6810.00 & \\
\hline & & \multicolumn{3}{|c|}{ Minimum } & \multicolumn{2}{|l|}{1.27} & \multicolumn{3}{|l|}{ Minimum } & 5.01 & \\
\hline & & \multicolumn{3}{|c|}{$\mathrm{N}$} & \multicolumn{2}{|l|}{25} & \multicolumn{3}{|l|}{$\mathrm{N}$} & 22 & \\
\hline
\end{tabular}




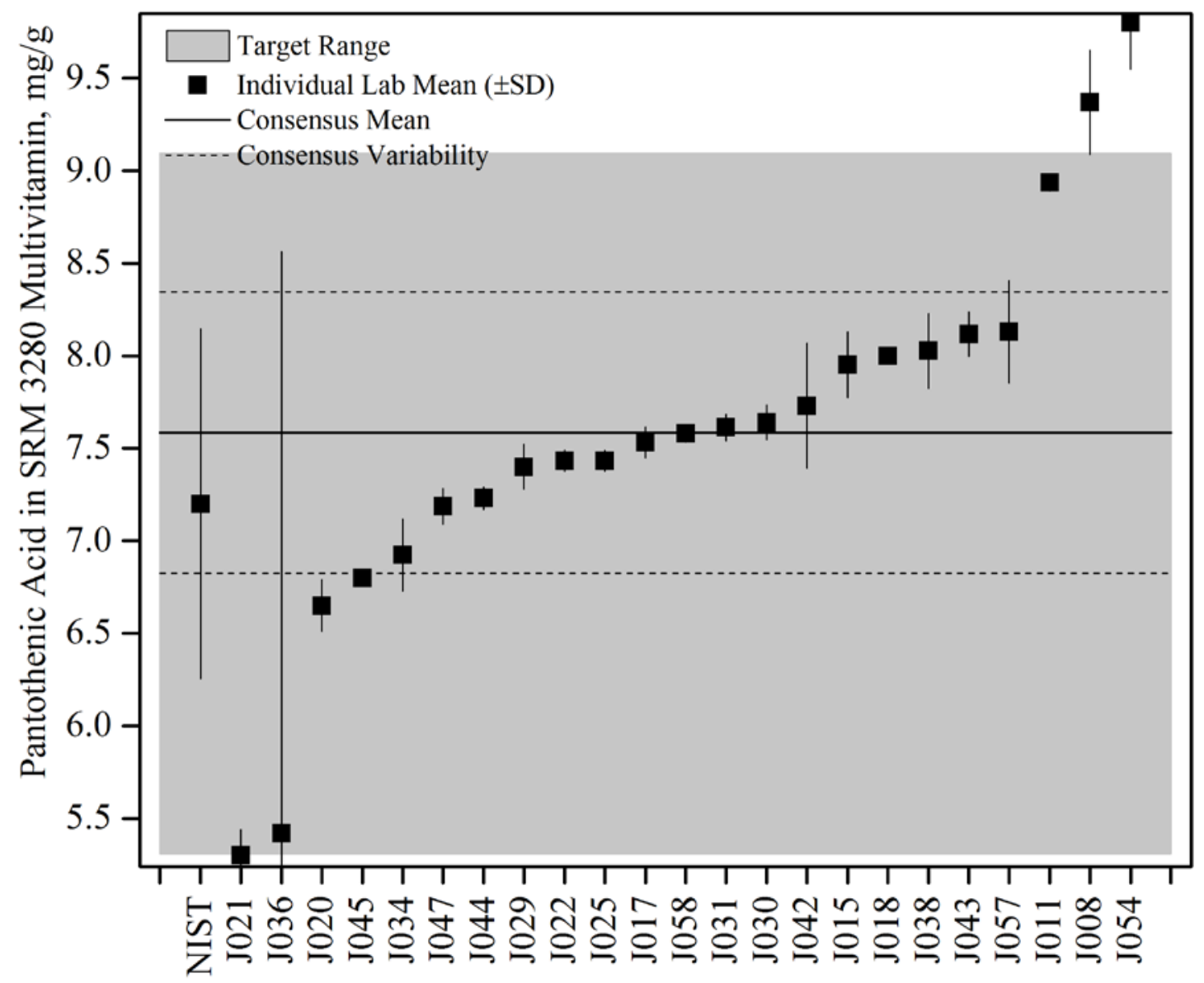

Figure 19. Pantothenic acid in SRM 3280 Multivitamin/Multielement Tablets (data summary view). In this view, individual laboratory data are plotted with the individual laboratory standard deviation (error bars). The black solid line represents the consensus mean, and the black dotted lines represent the consensus variability calculated as one standard deviation about the consensus mean. The gray shaded region represents the target zone for "acceptable” performance, which encompasses the NIST certified value bounded by twice its uncertainty $\left(U_{95}\right)$. 


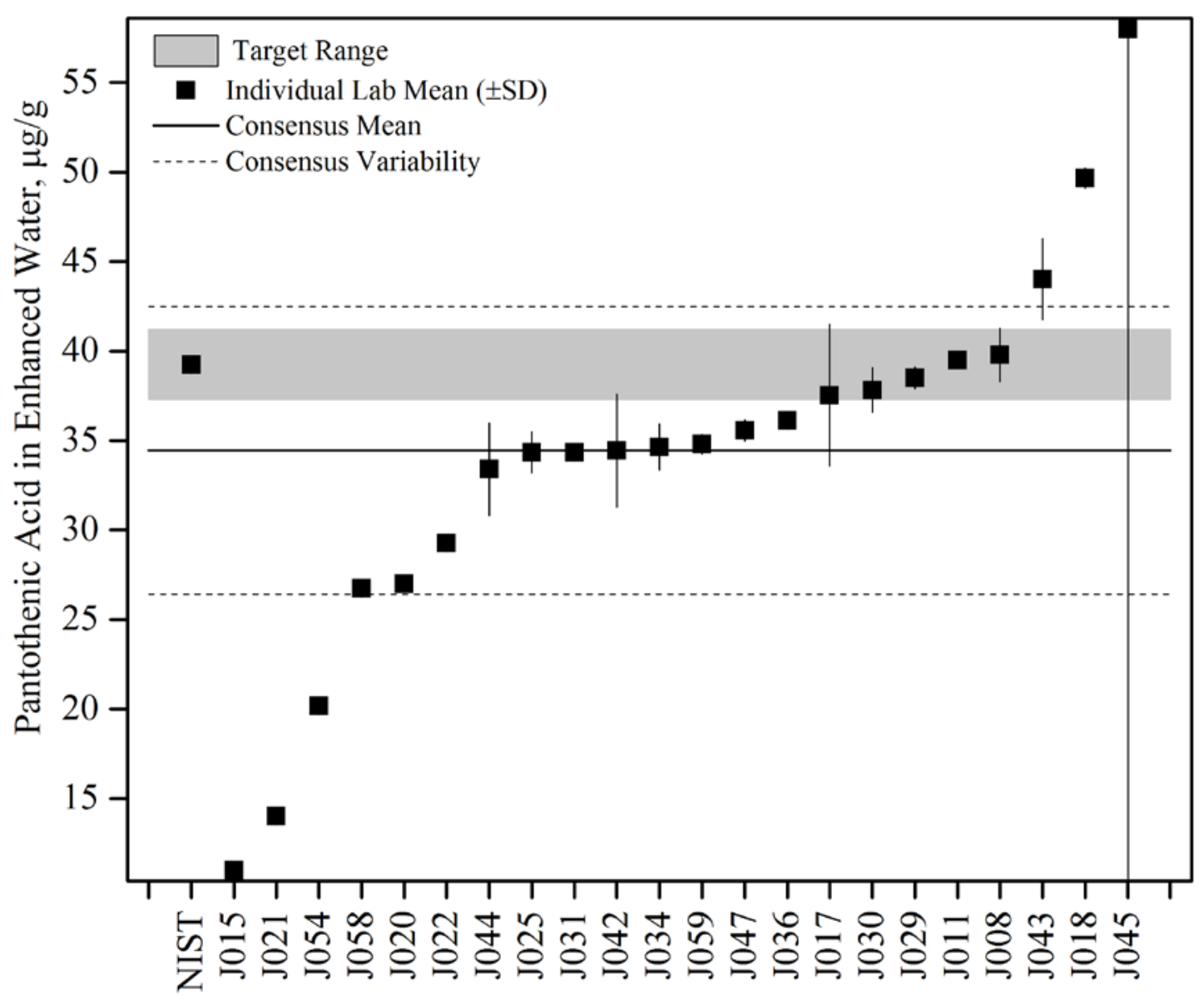

Figure 20. Pantothenic acid in enhanced water (data summary view). In this view, individual laboratory data are plotted with the individual laboratory standard deviation (error bars). The black solid line represents the consensus mean, and the black dotted lines represent the consensus variability calculated as one standard deviation about the consensus mean. The gray shaded region represents the target zone for "acceptable" performance, which encompasses the NIST value determined in triplicate by ID-LC/MS, bounded by an estimated relative uncertainty of $5 \%$. 


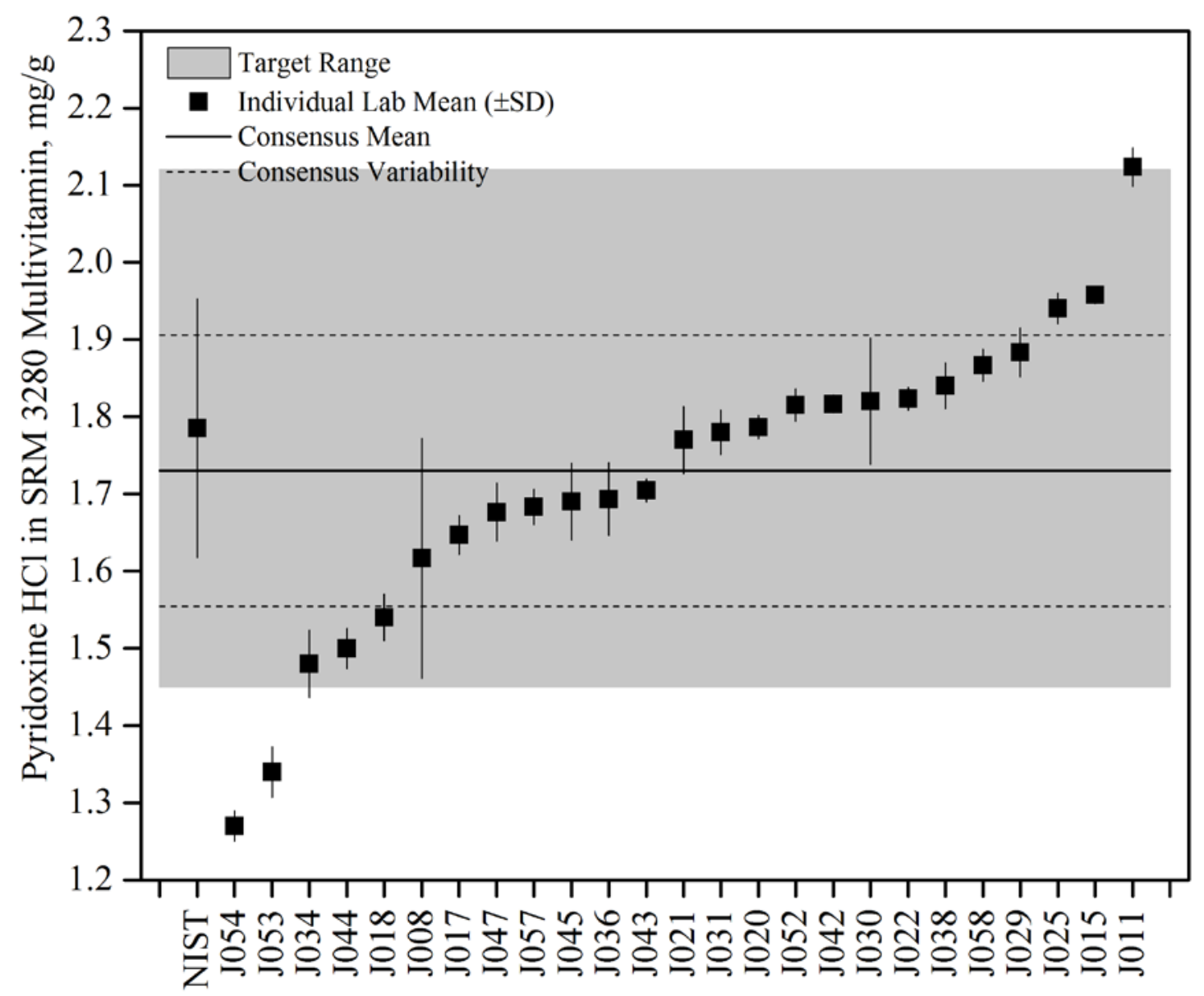

Figure 21. Pyridoxine hydrochloride in SRM 3280 Multivitamin/Multielement Tablets (data summary view). In this view, individual laboratory data are plotted with the individual laboratory standard deviation (error bars). The black solid line represents the consensus mean, and the black dotted lines represent the consensus variability calculated as one standard deviation about the consensus mean. The gray shaded region represents the target zone for "acceptable" performance, which encompasses the NIST certified value bounded by twice its uncertainty $\left(U_{95}\right)$. 


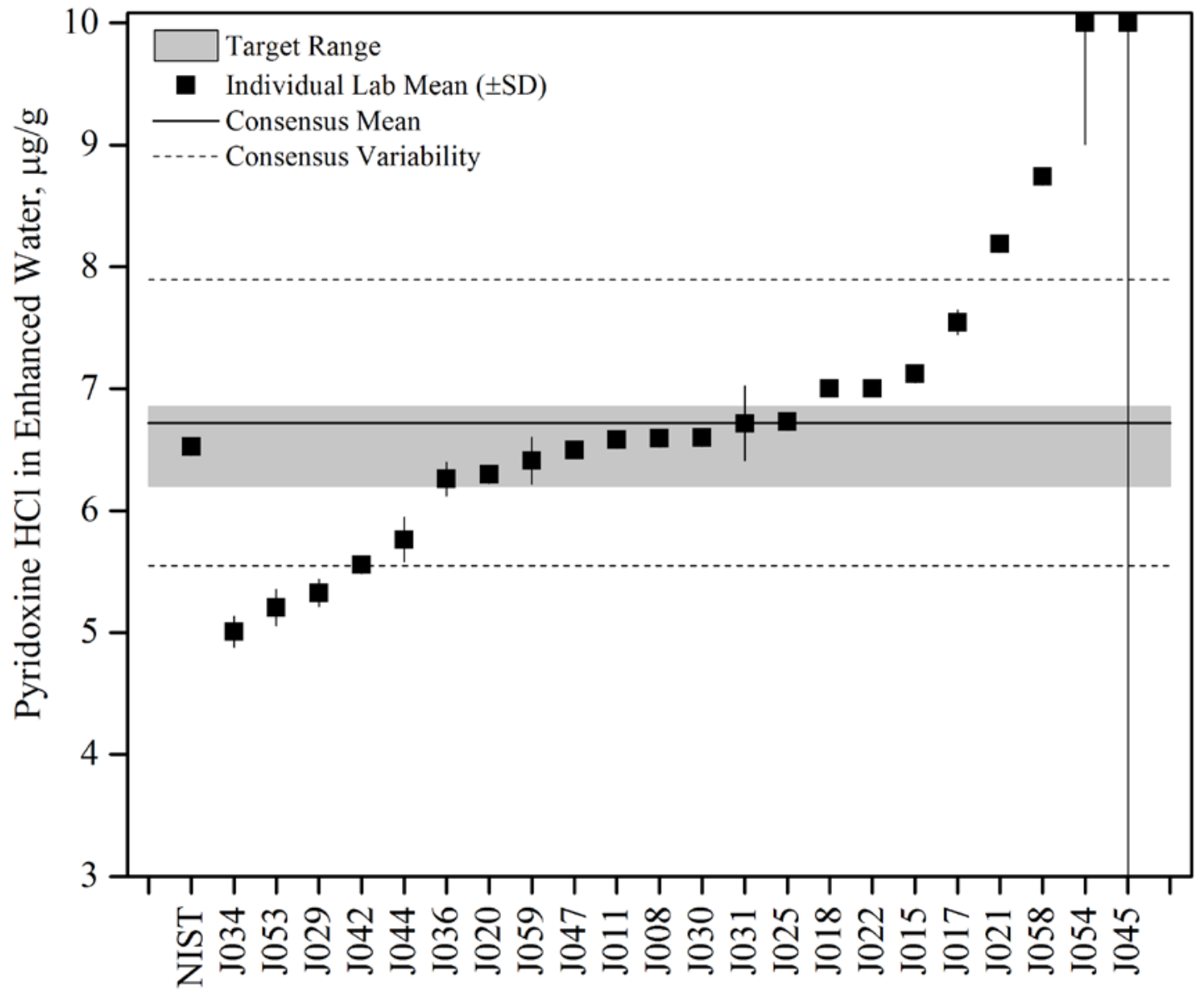

Figure 22. Pyridoxine hydrochloride in enhanced water (data summary view). In this view, individual laboratory data are plotted with the individual laboratory standard deviation (error bars). The black solid line represents the consensus mean, and the black dotted lines represent the consensus variability calculated as one standard deviation about the consensus mean. The gray shaded region represents the target zone for "acceptable” performance, which encompasses the NIST value determined in triplicate by ID-LC/MS, bounded by an estimated relative uncertainty of $5 \%$. 


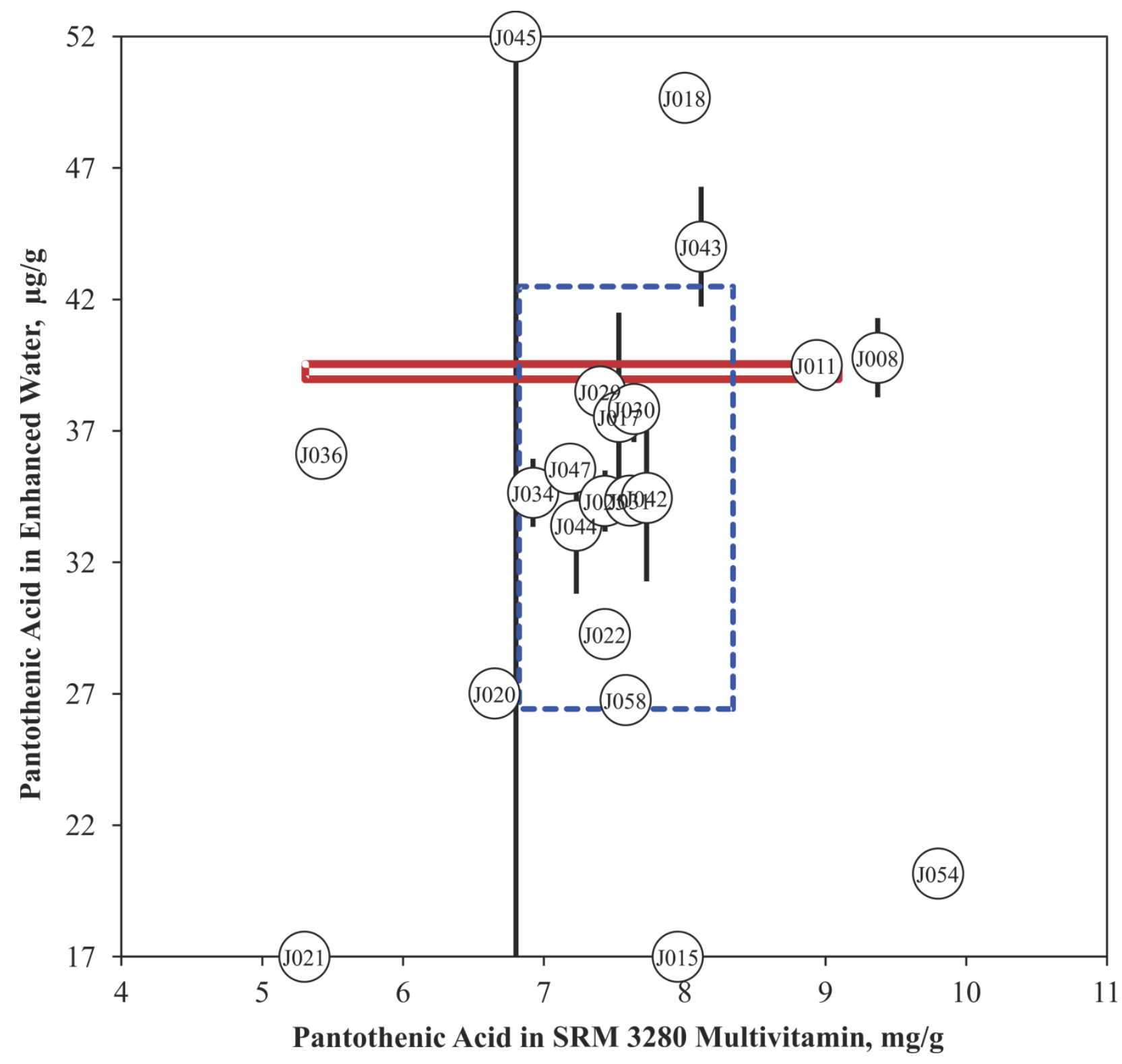

Figure 23. Pantothenic acid in SRM 3280 Multivitamin/Multielement Tablets and enhanced water (sample/control comparison view). In this view, the individual laboratory results for the control (SRM 3280) with a certified value for the analyte are compared to the results for an unknown (enhanced water). The solid red box represents the target zone for the control (x-axis) and unknown sample (y-axis). The dotted blue box represents the consensus zone for the control (x-axis) and the unknown sample (y-axis). 


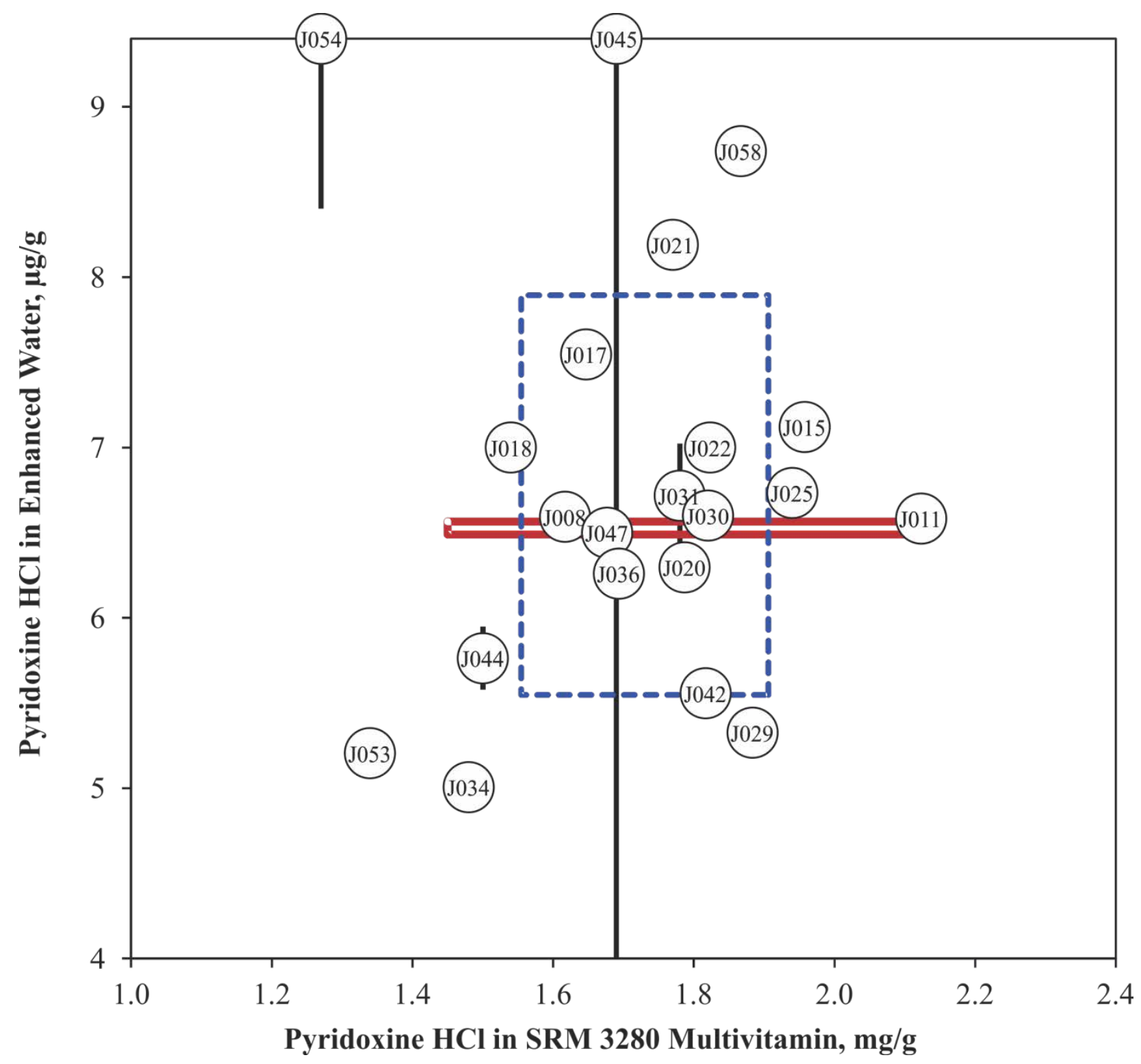

Figure 24. Pyridoxine hydrochloride in SRM 3280 Multivitamin/Multielement Tablets and enhanced water (sample/control comparison view). In this view, the individual laboratory results for the control (SRM 3280) with a certified value for the analyte are compared to the results for an unknown (enhanced water). The solid red box represents the target zone for the control (xaxis) and unknown sample (y-axis). The dotted blue box represents the consensus zone for the control (x-axis) and the unknown sample (y-axis). 


\section{VITAMIN E IN DIETARY SUPPLEMENTS}

\section{Study Overview}

In this study, participants were provided with one NIST SRM, SRM 3280 Multivitamin/ Multielement Tablets, and one bottle of commercially available enhanced water. Participants were asked to use in-house analytical methods to determine the mass fraction of vitamin $\mathrm{E}$ (as $\alpha$ tocopherol acetate) in each of the matrices and report values on an as-received basis.

\section{$\underline{\text { Sample Information }}$}

Multivitamin/Multielement Tablets. Participants were provided with one bottle containing 30 multivitamin/multielement tablets. Before use, participants were instructed to grind all 30 tablets, mix the resulting powder thoroughly, and use a sample size of at least $0.6 \mathrm{~g}$. Participants were asked to store the material at controlled room temperature, $10^{\circ} \mathrm{C}$ to $30{ }^{\circ} \mathrm{C}$, prepare three samples, and report three values from the single bottle provided. Approximate analyte levels were not reported to participants prior to the study. The NIST certified value and uncertainty for vitamin E (as $\alpha$-tocopherol acetate) in SRM 3280 was determined by LC/abs and LC/MS following solvent extraction, in combination with data from numerous collaborating laboratories. The certified value is reported in the table below as $\alpha$-tocopherol equivalents and as $\alpha$-tocopherol acetate, both on a dry-mass basis and after correction for moisture of the material $(1.37 \%)$.

$\begin{array}{ccc}\text { Analyte } & \begin{array}{c}\text { Certified Mass Fraction } \\ \text { in SRM } 3280(\mathrm{mg} / \mathrm{g}) \\ \text { (dry-mass basis) }\end{array} & \begin{array}{c}\text { Certified Mass Fraction } \\ \text { in SRM } 3280(\mathrm{mg} / \mathrm{g})\end{array} \\ \begin{array}{c}\text { (as-received basis) } \\ \text {-tocopherol equivalents }\end{array} & \begin{array}{c}21.4 \pm 3.5 \\ 23.5 \pm 3.8\end{array} & 23.1 \pm 3.5 \\ \alpha \text {-tocopherol acetate } & 23.2 \pm 3.8\end{array}$

Enhanced Water. Participants were provided with one $600 \mathrm{~mL}$ bottle of commercially available enhanced water. Before use, participants were instructed to thoroughly mix the contents of the bottle, and a sample size of at least $1.0 \mathrm{~mL}$ was recommended. Participants were asked to store the material at controlled room temperature, $10{ }^{\circ} \mathrm{C}$ to $30{ }^{\circ} \mathrm{C}$, prepare three samples, and report three values from the single bottle provided. Approximate analyte levels were not reported to participants prior to the study. Certified values are not available for this material.

\section{$\underline{\text { Study Results }}$}

- Thirty-two laboratories enrolled in this exercise and received samples. Twenty laboratories reported results for vitamin E in SRM 3280 (62 \% participation) and 18 laboratories reported results for vitamin $\mathrm{E}$ in enhanced water (56 \% participation).

- The consensus mean was within the target range for vitamin E in SRM 3280 with acceptable variability (9 \% RSD).

- The consensus variability was also acceptable for vitamin $\mathrm{E}$ in enhanced water (21\% RSD), a sample with a concentration 1000 times lower than that in the control material.

- A majority of the laboratories reported using solvent extraction (85 \%) as the sample preparation method. Some laboratories also reported using saponification (15 \%).

- A majority of the laboratories reported using LC/Abs (85 \%) as their instrumental method for analysis. Liquid chromatography/fluorescence (LC/FL) was also reported by some laboratories (15\%). 


\section{Technical Recommendations}

The following recommendations are based on results obtained by the participants in this study.

- When using saponification for sample preparation, it is important to determine that the saponification reaction is complete. Additionally, if an internal standard approach to calibration is used, the internal standard must be stable under the sample preparation conditions.

- Absorbance and fluorescence detection are both valuable tools for the determination of tocopherols. Fluorescence detection is especially sensitive and selective for $\alpha-, \beta-, \gamma-$, and $\delta$-tocopherol, however it is not very sensitive for the detection of $\alpha$-tocopherol acetate. In this study, if the samples were saponified and the analyte was converted to $\alpha$ tocopherol, fluorescence is an appropriate choice for detection.

- In general, the results reported for this study were very good, but there is a slight indication of calibration error in the sample/sample view. $\alpha$-tocopherol acetate and $\alpha$ tocopherol calibrant concentrations should be determined spectrophotometrically with a liquid chromatography purity correction. Also, be certain that the correct molar absorptivity is used.

- If laboratories would like further guidance on the use of calibrants traceable to molar absorptivity (rather than mass), please indicate the need. NIST staff will be happy to provide additional information and/or training as appropriate. 
Table 10. Individual data summary table (NIST) for $\alpha$-tocopherol acetate in dietary supplements.

\section{National Institute of Standards \& Technology}

\begin{tabular}{ccc} 
& Lab Code: & NIST \\
\hline Analyte & Sample & Units \\
\hline Vitamin E & Multivitamin & $\mathrm{mg} / \mathrm{g}$ \\
Vitamin E & Enhanced Water & $\mu \mathrm{g} / \mathrm{g}$
\end{tabular}

Exercise J - May 2013 - Vitamin E

\begin{tabular}{cccc}
\multicolumn{5}{c}{ 1. Your Results } \\
\hline $\mathrm{x}_{\mathrm{i}}$ & $\mathrm{s}_{\mathrm{i}}$ & $\mathrm{Z}_{\text {comm }}$ & $\mathrm{Z}_{\mathrm{NIST}}$ \\
\hline 23.2 & 3.8 & 0.1 & 0.0 \\
& & & \\
\hline
\end{tabular}

\begin{tabular}{ccc}
\multicolumn{3}{c}{ 2. Community } \\
\hline $\mathrm{N}$ & $\mathrm{X}^{*}$ & $\mathrm{~s}^{*}$ \\
\hline 20 & 23.0 & 2.2 \\
18 & 23.8 & 4.9 \\
\hline
\end{tabular}

\begin{tabular}{cc}
\multicolumn{2}{c}{ 3. Target } \\
\hline $\mathrm{X}_{\mathrm{NIST}}$ & $U_{95}$ \\
\hline 23.2 & 3.8
\end{tabular}

$\mathrm{x}_{\mathrm{i}}$ Mean of reported values

$\mathrm{s}_{\mathrm{i}}$ Standard deviation of reported values

$\mathrm{N}$ Number of quantitative values reported

$\mathrm{Z}_{\text {comm }} \mathrm{Z}$-score with respect to community consensus

$\mathrm{x}^{*}$ Robust mean of reported values

$\mathrm{x}_{\text {NIST }}$ NIST-assessed value

$U_{95} \pm 95 \%$ confidence interval about the assessed value or standard deviation $\left(\mathrm{s}_{\mathrm{NIST}}\right)$

$\mathrm{Z}_{\mathrm{NIST}} \mathrm{Z}$-score with respect to NIST value

s* Robust standard deviation 
Table 11. Data summary table for $\alpha$-tocopherol acetate in dietary supplements.

\begin{tabular}{|c|c|c|c|c|c|c|c|c|c|c|c|}
\hline & \multirow[b]{3}{*}{ Lab } & \multicolumn{10}{|c|}{ Tocopherol Acetate } \\
\hline & & \multicolumn{5}{|c|}{ SRM 3280 Multivitamin/Multielement Tablets (mg/g) } & \multicolumn{5}{|c|}{ Enhanced Water $(\mu \mathrm{g} / \mathrm{g})$} \\
\hline & & A & B & C & Avg & SD & A & B & $\mathbf{C}$ & Avg & SD \\
\hline \multirow{33}{*}{ 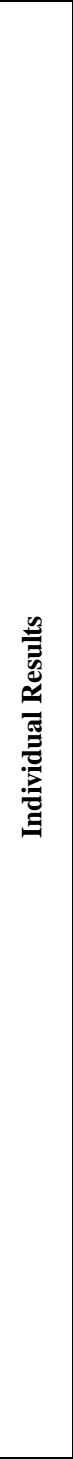 } & NIST & & & & 23.2 & 3.8 & & & & & \\
\hline & J002 & & & & & & & & & & \\
\hline & J004 & & & & & & & & & & \\
\hline & J008 & 22.4 & 22.2 & 23.3 & 22.6 & 0.6 & 17.6 & 18.6 & 18.6 & 18.3 & 0.6 \\
\hline & J011 & 18.9 & 18.1 & 18.6 & 18.5 & 0.4 & & & & & \\
\hline & J015 & 21.5 & 21.1 & 20.7 & 21.1 & 0.4 & 27.5 & 23.7 & 23.5 & 24.9 & 2.3 \\
\hline & J016 & 22.1 & 21.8 & 20.3 & 21.4 & 1.0 & 22.1 & 21.6 & 21.3 & 21.6 & 0.4 \\
\hline & J017 & 23.3 & 24.7 & 26.9 & 25.0 & 1.8 & 23.2 & 30.0 & 27.0 & 26.7 & 3.4 \\
\hline & J018 & & & & & & & & & & \\
\hline & J020 & 36.0 & 34.0 & 35.0 & 35.0 & 1.0 & 19.6 & 21.6 & 21.3 & 20.8 & 1.1 \\
\hline & J021 & 22.5 & 22.4 & 22.6 & 22.5 & 0.1 & 13.1 & 13.4 & 13.7 & 13.4 & 0.3 \\
\hline & J022 & 23.2 & 23.8 & 24.0 & 23.7 & 0.4 & 25.1 & 26.2 & 25.7 & 25.7 & 0.6 \\
\hline & J023 & & & & & & & & & & \\
\hline & J025 & 26.5 & 29.0 & 28.8 & 28.1 & 1.4 & 109.0 & 48.0 & 53.0 & 70.0 & 33.9 \\
\hline & J029 & 23.4 & 23.2 & 23.0 & 23.2 & 0.2 & 25.5 & 24.8 & 25.5 & 25.3 & 0.4 \\
\hline & J030 & & & & & & & & & & \\
\hline & J031 & 24.6 & 24.3 & 24.4 & 24.4 & 0.2 & & & & & \\
\hline & J036 & 23.9 & 23.8 & 23.9 & 23.9 & 0.1 & 25.8 & 27.4 & 27.5 & 26.9 & 0.9 \\
\hline & J037 & & & & & & & & & & \\
\hline & J038 & 26.1 & 26.0 & 25.1 & 25.7 & 0.5 & 28.6 & 30.6 & 27.4 & 28.9 & 1.6 \\
\hline & J039 & & & & & & & & & & \\
\hline & J042 & 21.1 & 21.2 & 21.2 & 21.1 & 0.0 & 26.0 & 24.4 & 28.0 & 26.1 & 1.8 \\
\hline & J043 & 20.6 & 21.2 & 21.0 & 20.9 & 0.3 & 19.2 & 21.1 & 18.5 & 19.6 & 1.3 \\
\hline & J044 & 22.9 & 23.6 & 23.1 & 23.2 & 0.4 & 26.4 & 19.5 & 22.2 & 22.7 & 3.5 \\
\hline & J045 & & & & & & & & & & \\
\hline & J047 & & & & & & & & & & \\
\hline & J048 & 20.1 & 21.6 & & 20.9 & 1.1 & 21.1 & 20.2 & & 20.7 & 0.6 \\
\hline & J052 & & & & & & & & & & \\
\hline & J053 & 20.3 & 23.1 & & 21.7 & 2.0 & 25.0 & 28.5 & & 26.7 & 2.5 \\
\hline & J054 & 24.2 & 24.1 & 24.6 & 24.3 & 0.3 & 30.0 & 27.1 & 29.3 & 28.8 & 1.5 \\
\hline & J057 & 20.7 & & & 20.7 & & & & & & \\
\hline & J058 & 21.9 & 21.2 & 22.8 & 22.0 & 0.8 & 19.1 & 15.7 & 16.4 & 17.1 & 1.8 \\
\hline & J059 & & & & & & & & & & \\
\hline \multirow{5}{*}{ 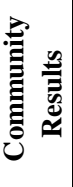 } & & \multirow{5}{*}{\multicolumn{3}{|c|}{$\begin{array}{l}\text { Consensus Mean } \\
\text { Consensus Standard Deviation } \\
\text { Maximum } \\
\text { Minimum } \\
\mathrm{N}\end{array}$}} & 23.0 & & \multirow{5}{*}{\multicolumn{3}{|c|}{$\begin{array}{l}\text { Consensus Mean } \\
\text { Consensus Standard D } \\
\text { Maximum } \\
\text { Minimum } \\
\mathrm{N} \\
\end{array}$}} & 23.8 & \\
\hline & & & & & 2.2 & & & & & 4.9 & \\
\hline & & & & & 35.0 & & & & & 70.0 & \\
\hline & & & & & 18.5 & & & & & 13.40 & \\
\hline & & & & & 20 & & & & & 18 & \\
\hline
\end{tabular}




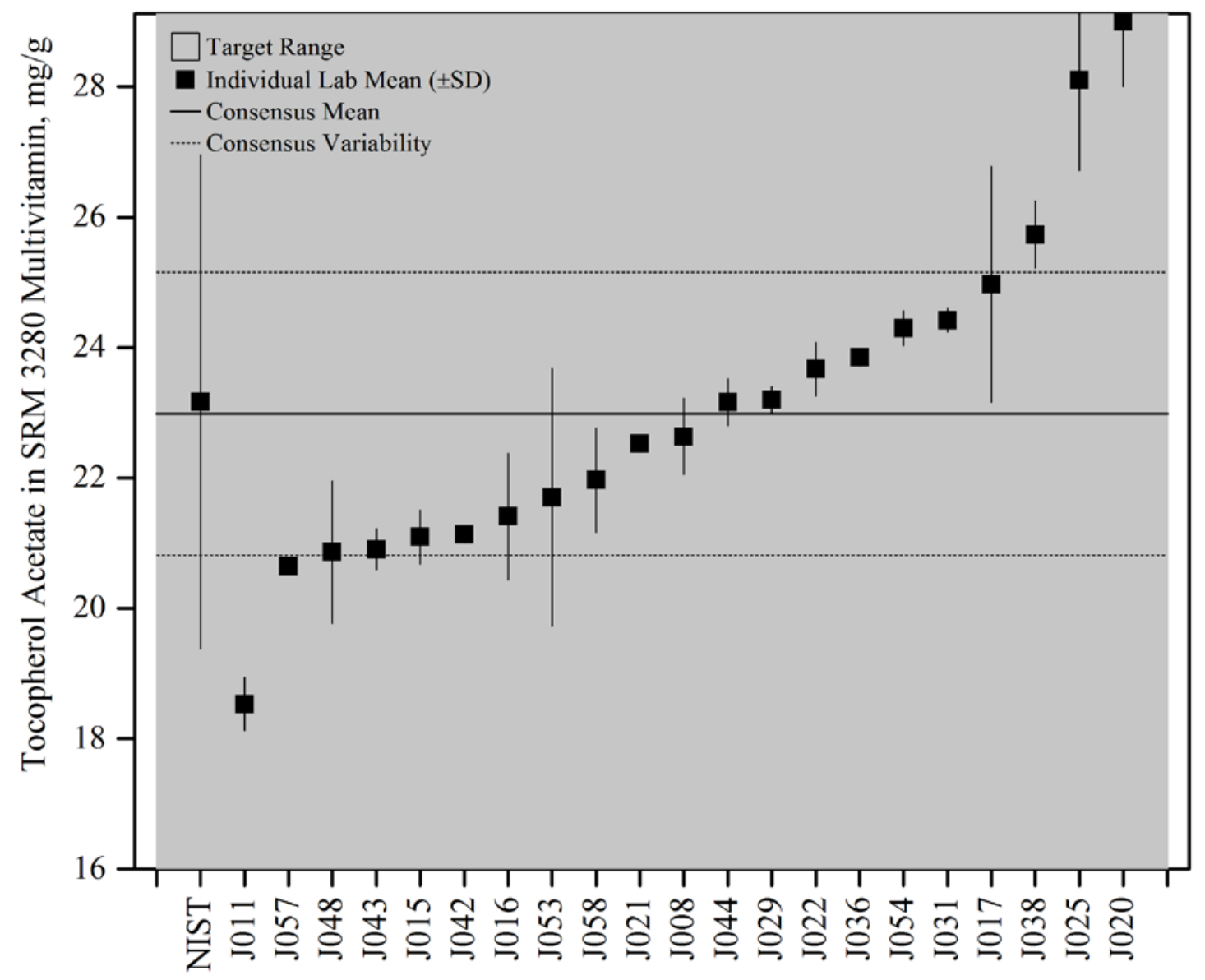

Figure 25. $\alpha$-Tocopherol acetate in SRM 3280 Multivitamin/Multielement Tablets (data summary view). In this view, individual laboratory data are plotted with the individual laboratory standard deviation (error bars). The black solid line represents the consensus mean, and the black dotted lines represent the consensus variability calculated as one standard deviation about the consensus mean. The gray shaded region represents the target zone for "acceptable" performance, which encompasses the NIST certified value bounded by twice its uncertainty $\left(U_{95}\right)$. 


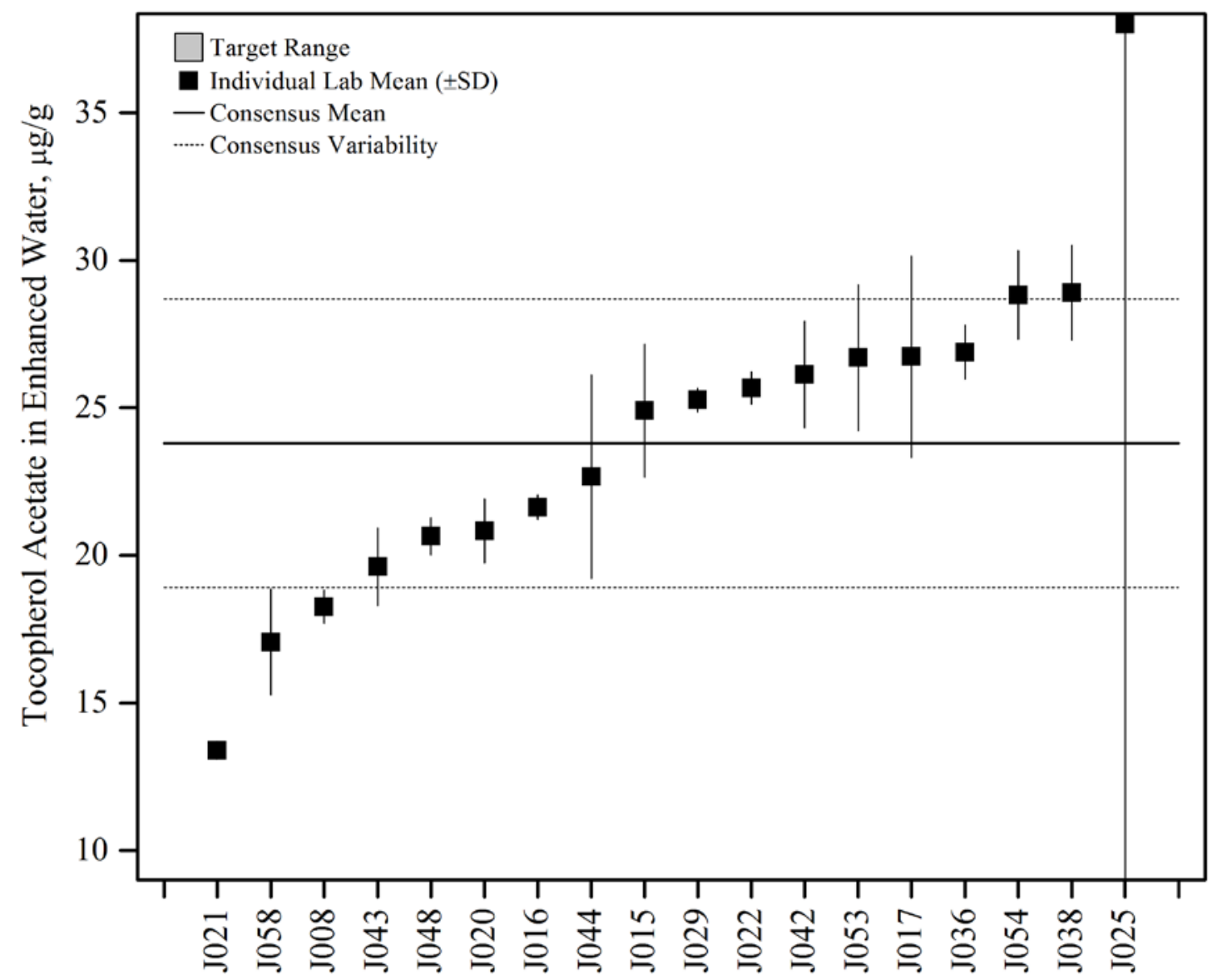

Figure 26. $\alpha$-Tocopherol acetate in enhanced water (data summary view). In this view, individual laboratory data are plotted with the individual laboratory standard deviation (error bars). The black solid line represents the consensus mean, and the black dotted lines represent the consensus variability calculated as one standard deviation about the consensus mean. 


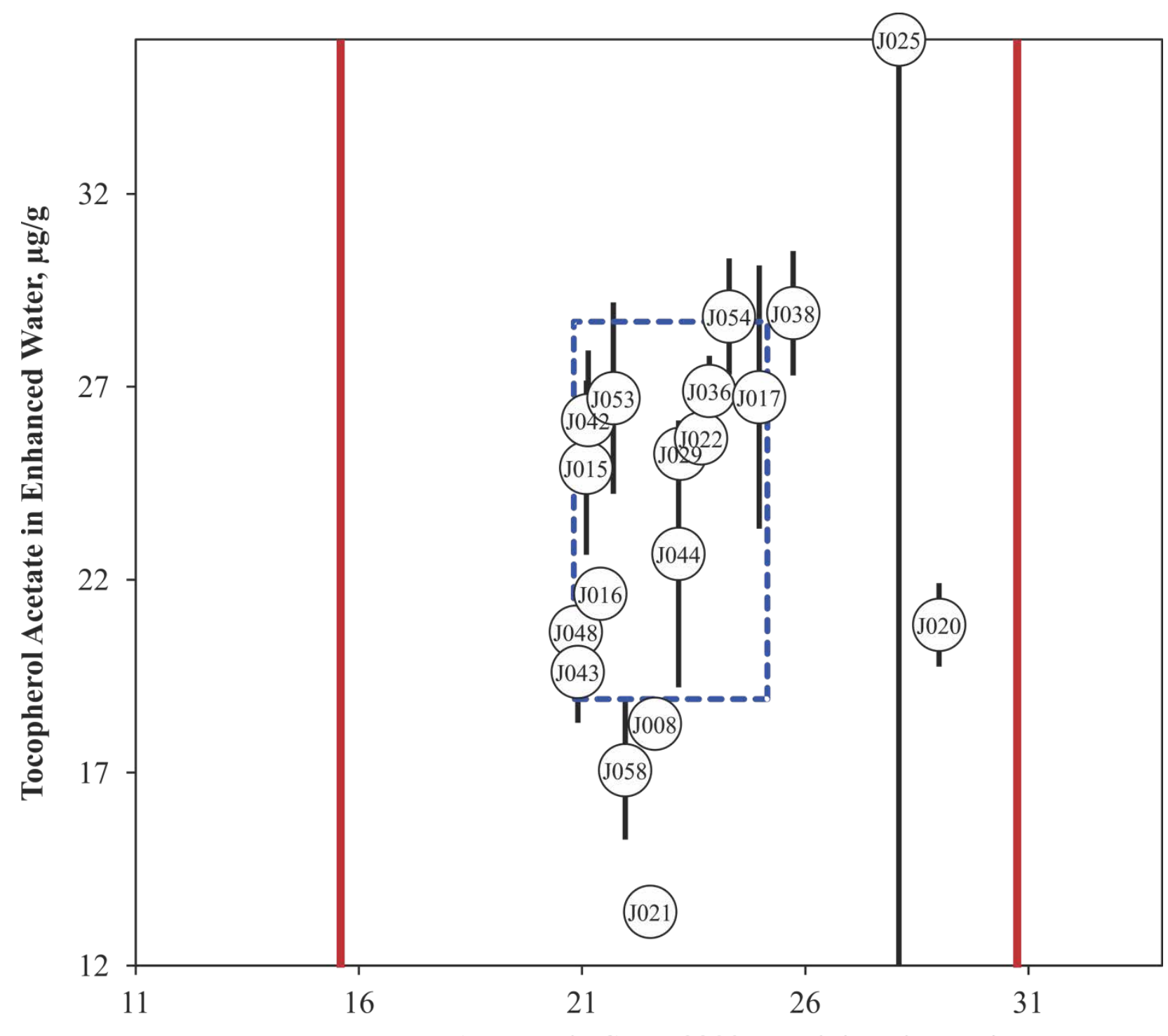

Tocopherol Acetate in SRM 3280 Multivitamin, mg/g

Figure 27. $\alpha$-Tocopherol acetate in SRM 3280 Multivitamin/Multielement Tablets and enhanced water (sample/control comparison view). In this view, the individual laboratory results for the control (multivitamin/multielement tablets) with a certified value for the analyte are compared to the results for an unknown (enhanced water). The solid red box represents the target zone for the control (x-axis). The dotted blue box represents the consensus zone for the control (x-axis) and the unknown sample (y-axis). 


\section{FATTY ACIDS IN BOTANICAL AND FISH OIL DIETARY SUPPLEMENTS}

\section{Study Overview}

In this study, participants were provided with two NIST SRMs, SRM 3274-2 Evening Primrose (Oenothera biennis) Oil and SRM 3275-2 Anchovy Oil. Participants were asked to use in-house analytical methods to determine the mass fractions of fatty acid methyl esters (FAMEs) in each of the matrices and report values on an as-received basis.

\section{Sample Information}

Evening Primrose Oil. Participants were provided with three ampoules, each containing $1.2 \mathrm{~mL}$ of evening primrose oil. The oil contained approximately $190 \mathrm{mg} / \mathrm{L}$ tert-butylhydroquinone (TBHQ) as an antioxidant and was packaged in amber glass ampoules under argon. Before use, participants were instructed to mix each ampoule thoroughly and a sample size of at least $0.5 \mathrm{~g}$ was recommended. Participants were asked to prepare one sample and report as many analytes as possible from each ampoule provided, and to store the oil under refrigeration at $0{ }^{\circ} \mathrm{C}$ to $4{ }^{\circ} \mathrm{C}$. Approximate analyte levels were not reported to participants prior to the study. The NIST certified and reference values and uncertainties in SRM 3274-2 were determined by by gas chromatography-flame ionization detection (GC-FID) and gas chromatography/mass spectrometry (GC/MS) following multiple methods of hydrolysis and derivatization, and are summarized in the table below.

Fish Oil. Participants were provided with three ampoules, each containing $1.2 \mathrm{~mL}$ of anchovy oil. The oil contained mixed natural tocopherols at a minimum of $1 \mathrm{mg} / \mathrm{g}$ as an antioxidant and was packaged in amber glass ampoules under argon. Before use, participants were instructed to mix each ampoule thoroughly and a sample size of at least $0.5 \mathrm{~g}$ was recommended. Participants were asked to prepare one sample and report as many analytes as possible from each ampoule provided, and to store the oil under refrigeration at $0{ }^{\circ} \mathrm{C}$ to $4{ }^{\circ} \mathrm{C}$. Approximate analyte levels were not reported to participants prior to the study. The NIST certified and reference values and uncertainties in SRM 3275-2 were determined by GC-FID and GC/MS following multiple methods of hydrolysis and derivatization, and are summarized in the table below.

\begin{tabular}{ccc} 
& \multicolumn{2}{c}{ Certified Mass Fraction } \\
Analyte & in SRM 3274-2 (mg/g as FAME) \\
\cline { 2 - 3 } Linoleic Acid & 745 & \pm 24 \\
$\alpha$ - Linoleic Acid & $1.61 \quad \pm 0.12$ \\
$\gamma$ - Linoleic Acid & $100.4 \quad \pm 4.1$ \\
Arachidonic Acid & $0.0221 \pm 0.0020$ \\
EPA & & \\
DHA & &
\end{tabular}

\begin{tabular}{|c|c|}
\hline $\begin{array}{r}\text { Certified } \\
\text { in SRM 3275- } \\
\end{array}$ & $\begin{array}{l}\text { Mass Fract } \\
2 \text { (mg/g as }\end{array}$ \\
\hline 3.00 & $\pm \quad 0.42$ \\
\hline 1.42 & $\pm \quad 0.12$ \\
\hline 0.507 & $\pm \quad 0.043$ \\
\hline 22.9 & $\pm \quad 1.0$ \\
\hline 394 & \pm 17 \\
\hline 187 & \pm 8 \\
\hline
\end{tabular}

Study Results

- Twenty-one laboratories enrolled in this exercise and received samples. Thirteen laboratories reported data for at least one FAME (62 \% participation).

- A majority of the laboratories reported using derivatization/methylation as the sample preparation method (92 \%). One laboratory also reported using hydrolysis as the sample preparation method. 
- A majority of the laboratories reported using GC-FID (85 \%) as their method for analysis. One laboratory reported using GC-MS and one laboratory reported using LC/absorbance.

- The consensus means for linoleic acid (LA) in both oils, $\alpha$-linolenic acid (ALA) in the botanical oil, and EPA and DHA in the fish oil were within the target ranges with acceptable variabilities (5\% to $21 \%$ RSD).

- The consensus mean for $\gamma$-linolenic acid (GLA) in the botanical oil was below the target range with low variability (5\% RSD).

- The consensus means for ALA, GLA, and AA in the fish oil were above the target range. The variabilities for ALA and GLA were high (31\% and $69 \%$ RSD, respectively), while the variability for AA was acceptable (16\% RSD).

Technical Recommendations

The following recommendations are based on results obtained by the participants in this study.

- With a small number of laboratories reporting data for these fatty acids, and a majority reporting use of the same or very similar methods, drawing extensive technical conclusions is difficult.

- Participants were asked to report concentrations for fatty acids as fatty acid methyl esters (FAMEs). In this case, FAMEs should be used as calibrants or non-esterified fatty acids should be carried through the entire sample preparation procedure (hydrolysis and derivatization) to improve quantitation.

- Knowledge of calibrant response when carried through the derivatization procedure is necessary. For example, at NIST, calibrants for EPA and DPA give response factors of 1.3 and 1.6, respectively, corresponding to $30 \%$ or $60 \%$ low bias in the quantitation of these compounds if not considered.

- Similarly, for those laboratories using GC-MS, quantitation for some compounds may be inaccurate as a result of non-unity response factors from EI fragmentation. 
Table 12. Individual data summary table (NIST) for fatty acids in botanical and fish oil dietary supplements.

\section{National Institute of Standards \& Technology}

\begin{tabular}{ccc} 
& & \\
& Lab Code: & NIST \\
\hline Analyte & Sample & Units \\
\hline Linoleic Acid & Primrose & $\mathrm{mg} / \mathrm{g}$ \\
Linoleic Acid & Fish & $\mathrm{mg} / \mathrm{g}$ \\
\hline$\alpha$-Linolenic Acid & Primrose & $\mathrm{mg} / \mathrm{g}$ \\
$\alpha$-Linolenic Acid & Fish & $\mathrm{mg} / \mathrm{g}$ \\
\hline$\gamma$-Linolenic Acid & Primrose & $\mathrm{mg} / \mathrm{g}$ \\
$\gamma$-Linolenic Acid & Fish & $\mathrm{mg} / \mathrm{g}$ \\
\hline Arachidonic Acid & Primrose & $\mathrm{mg} / \mathrm{g}$ \\
Arachidonic Acid & Fish & $\mathrm{mg} / \mathrm{g}$ \\
\hline EPA & Primrose & $\mathrm{mg} / \mathrm{g}$ \\
EPA & Fish & $\mathrm{mg} / \mathrm{g}$ \\
\hline DHA & Primrose & $\mathrm{mg} / \mathrm{g}$ \\
DHA & Fish & $\mathrm{mg} / \mathrm{g}$ \\
\hline
\end{tabular}

\section{Exercise J - May 2013 - Fatty Acids}

\begin{tabular}{cccc}
\multicolumn{4}{c}{ 1. Your Results } \\
\hline $\mathrm{x}_{\mathrm{i}}$ & $\mathrm{S}_{\mathrm{i}}$ & $\mathrm{Z}_{\text {comm }}$ & $\mathrm{Z}_{\mathrm{NIST}}$ \\
\hline 745 & 24 & 1.0 & 0.0 \\
3.00 & 0.42 & -0.2 & 0.0 \\
\hline 1.61 & 0.12 & 0.2 & 0.0 \\
1.42 & 0.12 & -0.8 & 0.0 \\
\hline 100 & 4 & 1.9 & 0.0 \\
0.507 & 0.043 & -0.9 & 0.0 \\
\hline 0.0221 & 0.0020 & & 0.0 \\
22.9 & 1.0 & -0.6 & 0.0 \\
\hline & & & \\
394 & 17 & 0.2 & 0.0 \\
\hline & & & \\
187 & 8 & -0.1 & 0.0 \\
\hline
\end{tabular}

\begin{tabular}{ccc}
\multicolumn{3}{c}{ 2. Community Results } \\
\hline $\mathrm{N}$ & $\mathrm{x}^{*}$ & $\mathrm{~s}^{*}$ \\
\hline 10 & 710 & 34 \\
7 & 3.13 & 0.57
\end{tabular}

\begin{tabular}{cc}
\multicolumn{2}{c}{ 3. Target } \\
\hline $\mathrm{x}_{\text {NIST }}$ & $U_{95}$ \\
\hline 745 & 24 \\
3.00 & 0.42 \\
\hline 1.61 & 0.12 \\
1.42 & 0.12 \\
\hline 100 & 4 \\
0.507 & 0.043 \\
\hline 0.0221 & 0.0020 \\
22.9 & 1.0 \\
\hline & \\
394 & 17 \\
\hline & \\
187 & 8
\end{tabular}

\begin{tabular}{ccc}
7 & 3.13 & 0.57 \\
\hline 8 & 1.54 & 0.33 \\
7 & 1.89 & 0.59 \\
\hline 11 & 91 & 5 \\
5 & 1.350 & 0.930 \\
\hline 1 & & \\
9 & 25.1 & 4.0 \\
\hline 1 & & \\
13 & 385 & 49 \\
\hline 0 & & \\
13 & 188 & 16 \\
\hline
\end{tabular}

$\begin{array}{r}187 \quad 8 \\ \hline\end{array}$

\footnotetext{
$\mathrm{x}_{\mathrm{i}}$ Mean of reported values

$s_{i}$ Standard deviation of reported values

$\mathrm{Z}_{\text {comm }}$ Z-score with respect to community consensus
}

$\mathrm{N}$ Number of quantitative values reported

$\mathrm{x}^{*}$ Robust mean of reported values

$\mathrm{Z}_{\mathrm{NIST}} \mathrm{Z}$-score with respect to NIST value $\mathrm{x}_{\text {NIST }} \quad$ NIST-assessed value

$U_{95} \pm 95 \%$ confidence interval about the assessed value or standard deviation ( $\left.\mathrm{s}_{\mathrm{NIST}}\right)$ 
Table 13. Data summary table for linoleic acid in botanical and fish oil dietary supplements.

\begin{tabular}{|c|c|c|c|c|c|c|c|c|c|c|c|}
\hline & \multirow[b]{3}{*}{ Lab } & \multicolumn{10}{|c|}{ Linoleic Acid } \\
\hline & & \multicolumn{5}{|c|}{ SRM 3274-2 Evening Primrose Oil (mg/g) } & \multicolumn{5}{|c|}{ SRM 3275-2 Fish Oil (mg/g) } \\
\hline & & A & B & C & Avg & SD & A & B & C & Avg & SD \\
\hline \multirow{22}{*}{ 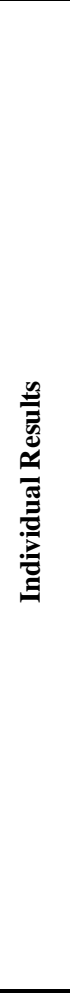 } & NIST & & & & 745 & 24 & & & & 3.00 & 0.42 \\
\hline & J002 & & & & & & & & & & \\
\hline & J004 & & & & & & & & & & \\
\hline & J007 & & & & & & & & & & \\
\hline & J009 & & & & & & & & & & \\
\hline & J011 & 668 & 663 & 657 & 663 & 6 & & & & & \\
\hline & J016 & & & & & & & & & & \\
\hline & J019 & 755 & 756 & 756 & 755 & 1 & 3.59 & 3.18 & 3.04 & 3.27 & 0.29 \\
\hline & J020 & & & & & & & & & & \\
\hline & J023 & & & & & & & & & & \\
\hline & J025 & & & & & & & & & & \\
\hline & J027 & 739 & 738 & 739 & 739 & 1 & 3.28 & 3.30 & 3.28 & 3.29 & 0.01 \\
\hline & J029 & 717 & 718 & 718 & 718 & 1 & 3.05 & 3.03 & 3.07 & 3.05 & 0.02 \\
\hline & J031 & 714 & 714 & 719 & 716 & 3 & & & & & \\
\hline & J036 & 714 & 696 & 710 & 707 & 9 & 2.35 & 2.28 & 2.25 & 2.29 & 0.05 \\
\hline & J038 & & & & & & & & & & \\
\hline & $\mathrm{J} 040$ & 706 & 706 & 706 & 706 & 0 & 5.00 & 5.00 & 5.00 & 5.00 & 0.00 \\
\hline & J043 & 726 & 709 & 714 & 716 & 9 & & & & & \\
\hline & J053 & 632 & 630 & 635 & 632 & 3 & 3.06 & 2.95 & 2.89 & 2.97 & 0.09 \\
\hline & J054 & & & & & & & & & & \\
\hline & J057 & 719 & 720 & 719 & 719 & 0 & 3.06 & 3.07 & 3.08 & 3.07 & 0.01 \\
\hline & J059 & & & & & & & & & & \\
\hline \multirow{5}{*}{ 色 } & & \multirow{5}{*}{\multicolumn{3}{|c|}{$\begin{array}{l}\text { Consensus Mean } \\
\text { Consensus Standard Deviation } \\
\text { Maximum } \\
\text { Minimum } \\
\mathrm{N}\end{array}$}} & 710 & & \multirow{5}{*}{\multicolumn{3}{|c|}{$\begin{array}{l}\text { Consensus Mean } \\
\text { Consensus Standard D } \\
\text { Maximum } \\
\text { Minimum } \\
\mathrm{N}\end{array}$}} & 3.13 & \\
\hline & & & & & 34 & & & & & 0.57 & \\
\hline & & & & & 755 & & & & & 5.00 & \\
\hline & & & & & 632 & & & & & 2.29 & \\
\hline & & & & & 10 & & & & & 7 & \\
\hline
\end{tabular}


Table 14. Data summary table for $\alpha$-linolenic acid in botanical and fish oil dietary supplements.

\begin{tabular}{|c|c|c|c|c|c|c|c|c|c|c|c|}
\hline & \multirow[b]{3}{*}{ Lab } & \multicolumn{10}{|c|}{$\alpha$-Linolenic Acid } \\
\hline & & \multicolumn{5}{|c|}{ SRM 3274-2 Evening Primrose Oil (mg/g) } & \multicolumn{5}{|c|}{ SRM 3275-2 Fish Oil (mg/g) } \\
\hline & & A & B & $\mathbf{C}$ & Avg & SD & A & B & C & Avg & SD \\
\hline \multirow{22}{*}{ 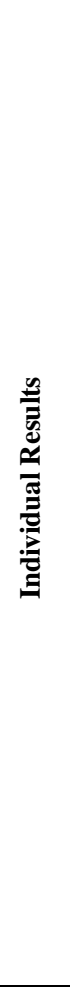 } & NIST & & & & 1.61 & 0.12 & & & & 1.42 & 0.12 \\
\hline & J002 & & & & & & & & & & \\
\hline & J004 & & & & & & & & & & \\
\hline & J007 & & & & & & & & & & \\
\hline & J009 & & & & & & & & & & \\
\hline & J011 & & & & & & & & & & \\
\hline & J016 & & & & & & & & & & \\
\hline & J019 & 1.25 & 1.23 & 1.30 & 1.26 & 0.04 & 1.69 & 1.38 & 1.46 & 1.51 & 0.16 \\
\hline & J020 & & & & & & & & & & \\
\hline & J023 & & & & & & & & & & \\
\hline & J025 & & & & & & & & & & \\
\hline & J027 & 1.69 & 1.68 & 1.70 & 1.69 & 0.01 & 1.87 & 1.82 & 1.81 & 1.83 & 0.04 \\
\hline & J029 & 1.59 & 1.56 & 1.62 & 1.59 & 0.03 & 1.42 & 1.45 & 1.43 & 1.43 & 0.02 \\
\hline & J031 & & & & & & & & & & \\
\hline & J036 & 1.60 & 1.54 & 1.59 & 1.58 & 0.03 & 2.57 & 2.53 & 2.50 & 2.53 & 0.04 \\
\hline & J038 & & & & & & & & & & \\
\hline & J040 & 2.00 & 2.00 & 2.00 & 2.00 & 0.00 & 2.00 & 2.00 & 2.00 & 2.00 & 0.00 \\
\hline & J043 & 1.14 & 1.21 & 1.01 & 1.12 & 0.10 & & & & & \\
\hline & J053 & 1.36 & 1.32 & 1.39 & 1.36 & 0.04 & 1.30 & 1.33 & 1.30 & 1.31 & 0.02 \\
\hline & J054 & & & & & & & & & & \\
\hline & J057 & 1.76 & 1.76 & 1.75 & 1.76 & 0.01 & 2.59 & 2.61 & 2.62 & 2.61 & 0.02 \\
\hline & J059 & & & & & & & & & & \\
\hline \multirow{5}{*}{ 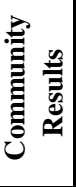 } & & \multirow{5}{*}{\multicolumn{3}{|c|}{$\begin{array}{l}\text { Consensus Mean } \\
\text { Consensus Standard Deviation } \\
\text { Maximum } \\
\text { Minimum } \\
\mathrm{N}\end{array}$}} & 1.54 & & \multirow{5}{*}{\multicolumn{3}{|c|}{$\begin{array}{l}\text { Consensus Mean } \\
\text { Consensus Standard Deviation } \\
\text { Maximum } \\
\text { Minimum } \\
\text { N }\end{array}$}} & 1.89 & \\
\hline & & & & & 0.33 & & & & & 0.59 & \\
\hline & & & & & 2.00 & & & & & 2.61 & \\
\hline & & & & & 1.12 & & & & & 1.31 & \\
\hline & & & & & 8 & & & & & 7 & \\
\hline
\end{tabular}


Table 15. Data summary table for $\gamma$-linolenic acid in botanical and fish oil dietary supplements.

\begin{tabular}{|c|c|c|c|c|c|c|c|c|c|c|c|}
\hline & \multirow[b]{3}{*}{ Lab } & \multicolumn{10}{|c|}{$\gamma$-Linolenic Acid } \\
\hline & & \multicolumn{5}{|c|}{ SRM 3274-2 Evening Primrose Oil (mg/g) } & \multicolumn{5}{|c|}{ SRM 3275-2 Fish Oil (mg/g) } \\
\hline & & A & B & C & Avg & SD & A & B & C & Avg & SD \\
\hline \multirow{22}{*}{ 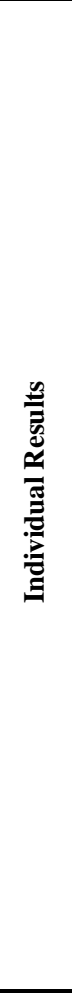 } & NIST & & & & 100.4 & 4.1 & & & & 0.507 & 0.043 \\
\hline & J002 & & & & & & & & & & \\
\hline & J004 & & & & & & & & & & \\
\hline & J007 & & & & & & & & & & \\
\hline & J009 & & & & & & & & & & \\
\hline & J011 & 73.9 & 74.0 & 73.4 & 73.8 & 0.3 & & & & & \\
\hline & J016 & & & & & & & & & & \\
\hline & J019 & 89.7 & 90.3 & 90.7 & 90.2 & 0.5 & & & & & \\
\hline & J020 & 89.0 & 89.0 & 89.0 & 89.0 & 0.0 & & & & & \\
\hline & J023 & & & & & & & & & & \\
\hline & J025 & & & & & & & & & & \\
\hline & J027 & 97.4 & 97.1 & 97.2 & 97.2 & 0.1 & 0.429 & 0.442 & 0.450 & 0.440 & 0.011 \\
\hline & J029 & 93.0 & 93.0 & 93.0 & 93.0 & 0.0 & & & & & \\
\hline & J031 & 92.5 & 92.6 & 93.2 & 92.8 & 0.4 & & & & & \\
\hline & J036 & 92.5 & 90.2 & 91.9 & 91.5 & 1.2 & 0.899 & 0.905 & 0.881 & 0.895 & 0.012 \\
\hline & J038 & & & & & & & & & & \\
\hline & J040 & 91.0 & 91.0 & 91.0 & 91.0 & 0.0 & 1.000 & 1.000 & 1.000 & 1.000 & 0.000 \\
\hline & J043 & 102.5 & 90.8 & 93.2 & 95.5 & 6.2 & & & & & \\
\hline & J053 & 80.4 & 79.3 & 80.0 & 79.9 & 0.6 & 2.126 & 2.000 & 2.090 & 2.072 & 0.065 \\
\hline & J054 & & & & & & & & & & \\
\hline & J057 & 94.4 & 94.4 & 94.4 & 94.4 & 0.0 & 2.350 & 2.360 & 2.360 & 2.357 & 0.006 \\
\hline & J059 & & & & & & & & & & \\
\hline \multirow{5}{*}{ 总 } & & \multirow{5}{*}{\multicolumn{3}{|c|}{$\begin{array}{l}\text { Consensus Mean } \\
\text { Consensus Standard Deviation } \\
\text { Maximum } \\
\text { Minimum } \\
\mathrm{N}\end{array}$}} & 91.1 & & \multirow{5}{*}{\multicolumn{3}{|c|}{$\begin{array}{l}\text { Consensus Mean } \\
\text { Consensus Standard Deviation } \\
\text { Maximum } \\
\text { Minimum } \\
\mathrm{N}\end{array}$}} & 1.353 & \\
\hline & & & & & 4.9 & & & & & 0.930 & \\
\hline & & & & & 97.2 & & & & & 2.357 & \\
\hline & & & & & 73.8 & & & & & 0.440 & \\
\hline & & & & & 11 & & & & & 5 & \\
\hline
\end{tabular}


Table 16. Data summary table for arachidonic acid in botanical and fish oil dietary supplements.

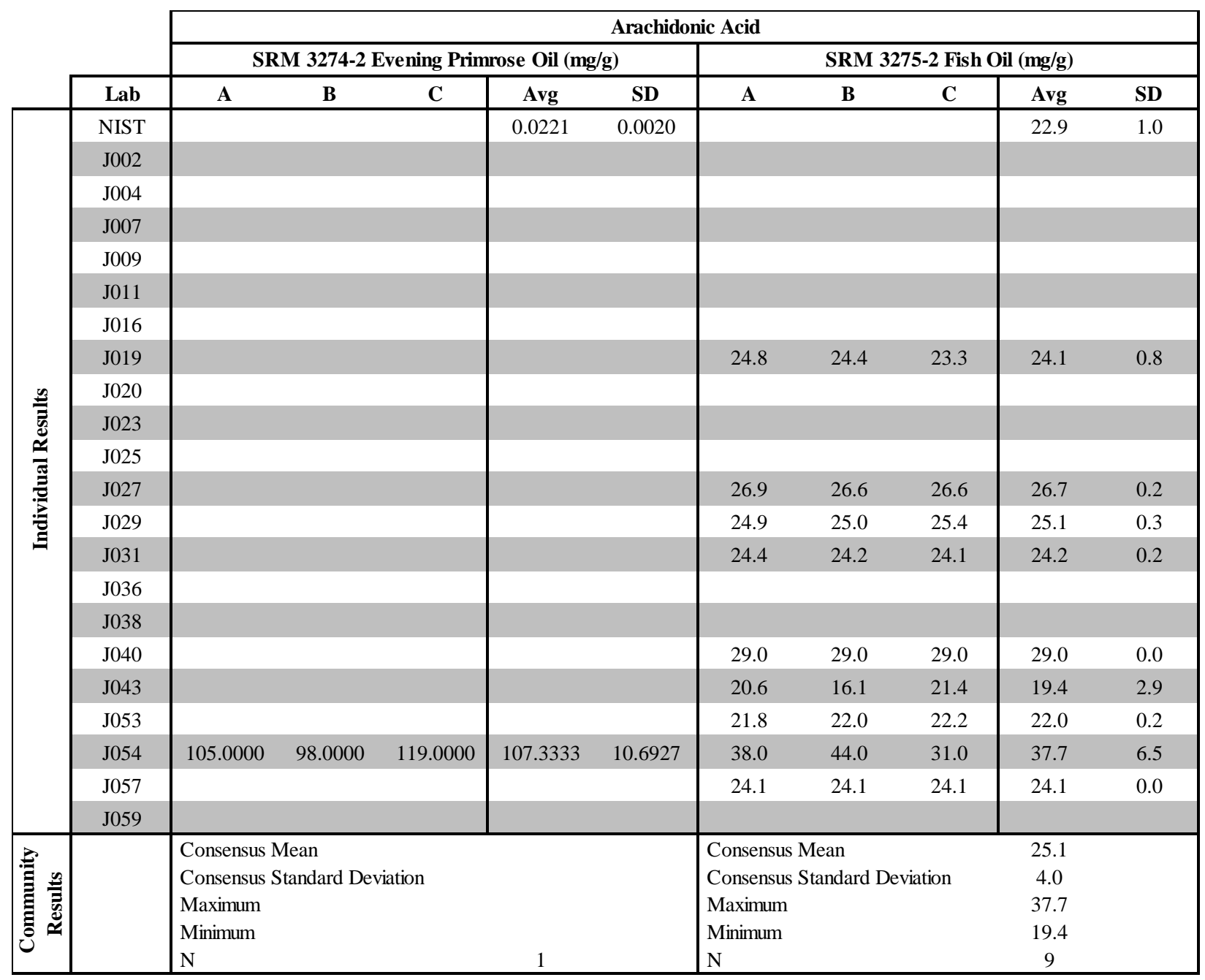


Table 17. Data summary table for EPA in botanical and fish oil dietary supplements.

\begin{tabular}{|c|c|c|c|c|c|c|c|c|c|c|c|}
\hline & \multirow[b]{3}{*}{ Lab } & \multicolumn{10}{|c|}{ EPA } \\
\hline & & \multicolumn{5}{|c|}{ SRM 3274-2 Evening Primrose Oil (mg/g) } & \multicolumn{5}{|c|}{ SRM 3275-2 Fish Oil (mg/g) } \\
\hline & & A & B & C & Avg & SD & A & B & C & Avg & SD \\
\hline \multirow{22}{*}{ 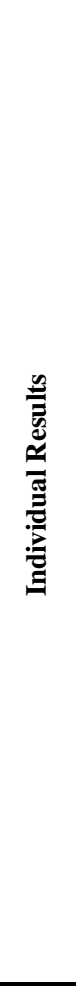 } & NIST & & & & & & & & & 394 & 17 \\
\hline & $\mathrm{J} 002$ & & & & & & & & & & \\
\hline & $\mathrm{J} 004$ & & & & & & & & & & \\
\hline & $\mathrm{J} 007$ & & & & & & & & & & \\
\hline & J009 & & & & & & & & & & \\
\hline & J011 & & & & & & 354 & 359 & 362 & 358 & 4 \\
\hline & J016 & & & & & & & & & & \\
\hline & J019 & 1.70 & 1.12 & 1.06 & 1.29 & 0.35 & 71 & 72 & 74 & 72 & 2 \\
\hline & J020 & & & & & & 368 & 370 & 375 & 371 & 4 \\
\hline & J023 & & & & & & 443 & 445 & 453 & 447 & 5 \\
\hline & J025 & & & & & & & & & & \\
\hline & J027 & & & & & & 412 & 408 & 408 & 409 & 3 \\
\hline & J029 & & & & & & 384 & 386 & 385 & 385 & 1 \\
\hline & J031 & & & & & & 386 & 385 & 384 & 385 & 1 \\
\hline & J036 & & & & & & 387 & 385 & 380 & 384 & 4 \\
\hline & J038 & & & & & & & & & & \\
\hline & J040 & & & & & & 467 & 457 & 459 & 461 & 5 \\
\hline & J043 & & & & & & 374 & 364 & 389 & 375 & 12 \\
\hline & J053 & & & & & & 403 & 412 & 410 & 408 & 5 \\
\hline & J054 & & & & & & 174 & 209 & 205 & 196 & 19 \\
\hline & J057 & & & & & & 400 & 400 & 400 & 400 & 0 \\
\hline & J059 & & & & & & & & & & \\
\hline \multirow{5}{*}{ 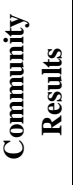 } & & \multirow{5}{*}{\multicolumn{5}{|c|}{$\begin{array}{l}\text { Consensus Mean } \\
\text { Consensus Standard Deviation } \\
\text { Maximum } \\
\text { Minimum } \\
\mathrm{N}\end{array}$}} & \multirow{5}{*}{\multicolumn{3}{|c|}{$\begin{array}{l}\text { Consensus Mean } \\
\text { Consensus Standard Deviation } \\
\text { Maximum } \\
\text { Minimum } \\
\text { N }\end{array}$}} & 385 & \\
\hline & & & & & & & & & & 49 & \\
\hline & & & & & & & & & & 461 & \\
\hline & & & & & & & & & & 72 & \\
\hline & & & & & & & & & & 13 & \\
\hline
\end{tabular}


Table 18. Data summary table for DHA in botanical and fish oil dietary supplements.

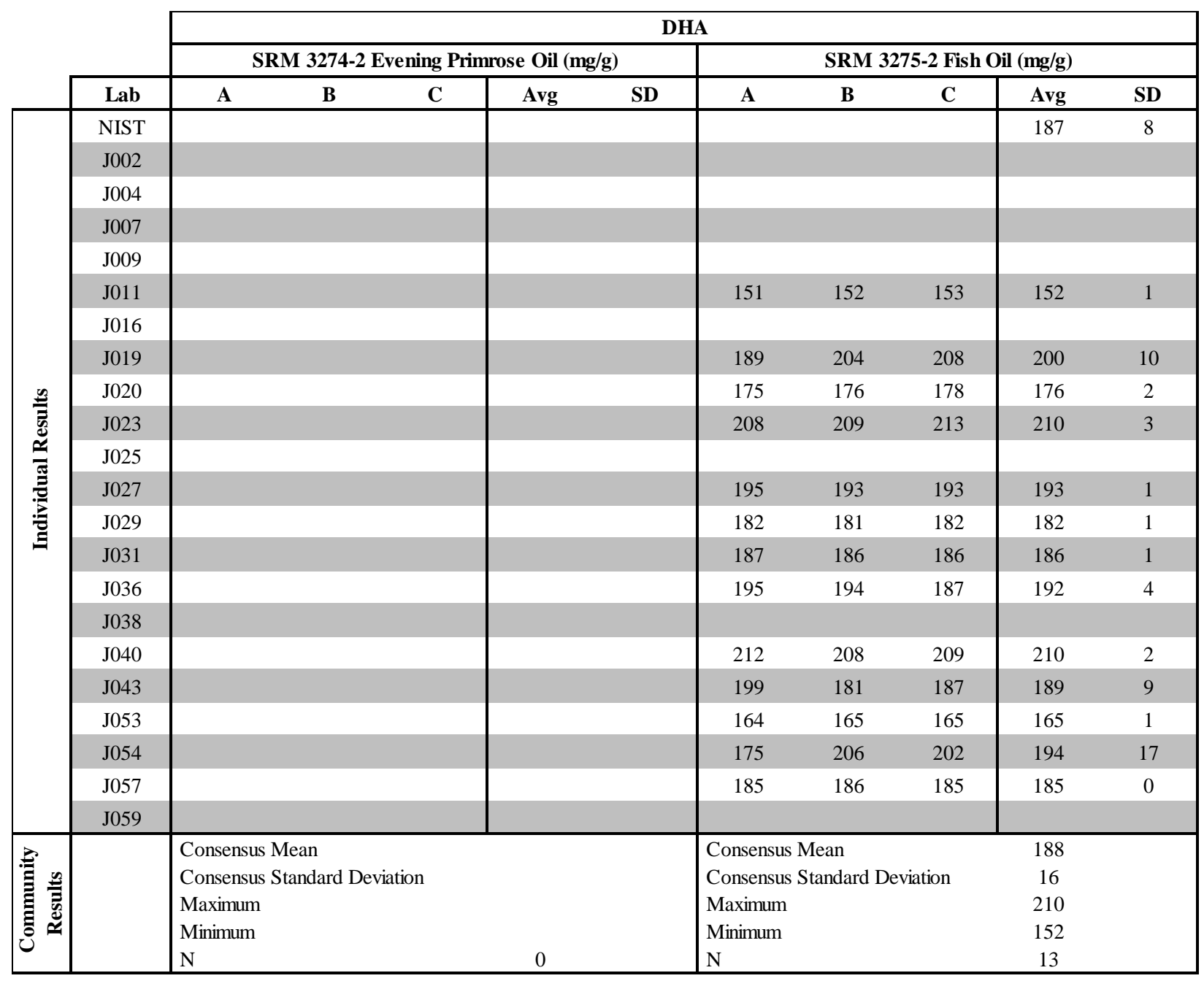




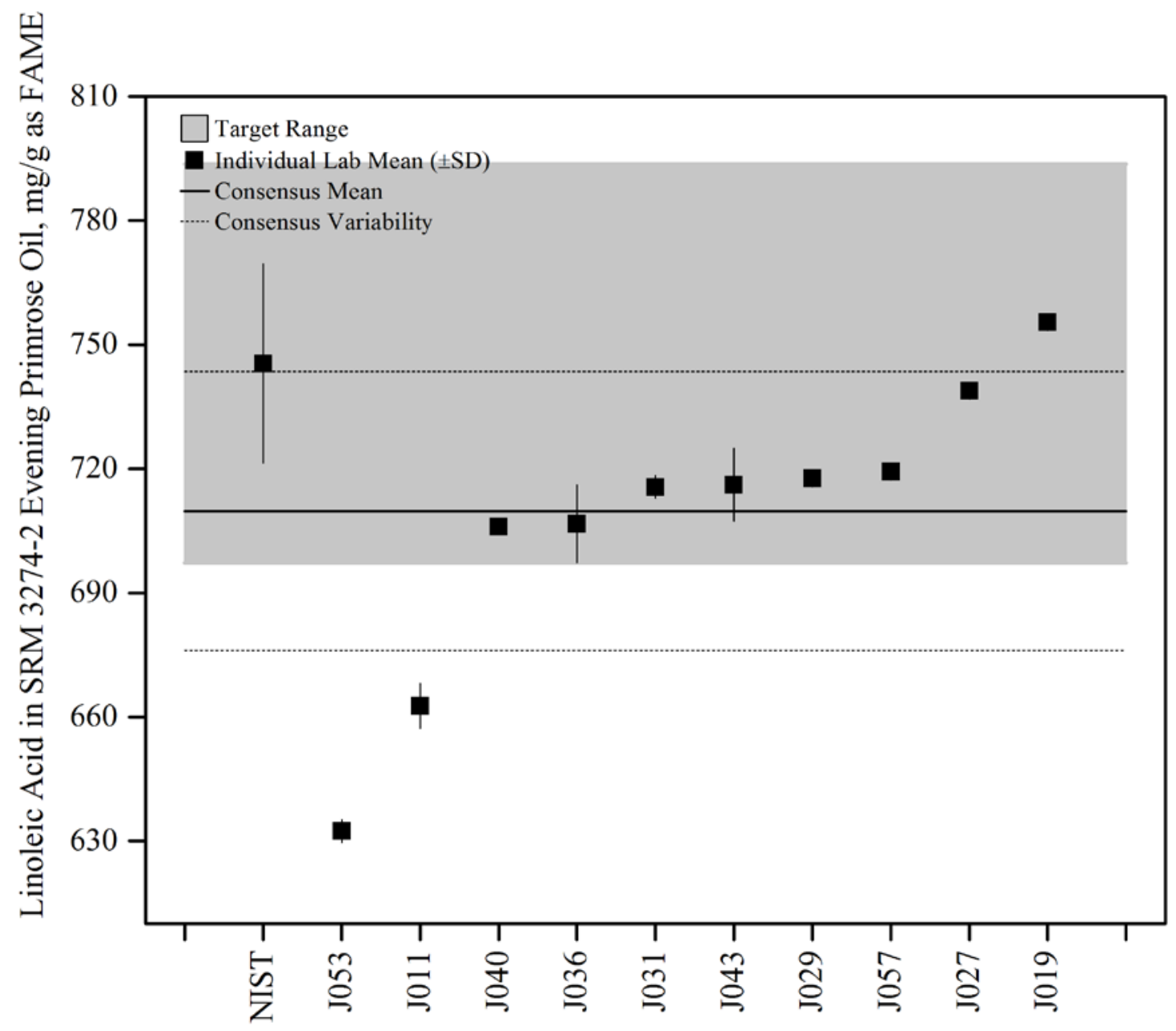

Figure 28. Linoleic acid in SRM 3274-2 Evening Primrose Oil (data summary view). In this view, individual laboratory data are plotted with the individual laboratory standard deviation (error bars). The black solid line represents the consensus mean, and the black dotted lines represent the consensus variability calculated as one standard deviation about the consensus mean. The gray shaded region represents the target zone for "acceptable" performance, which encompasses the NIST certified value bounded by twice its uncertainty $\left(U_{95}\right)$. 


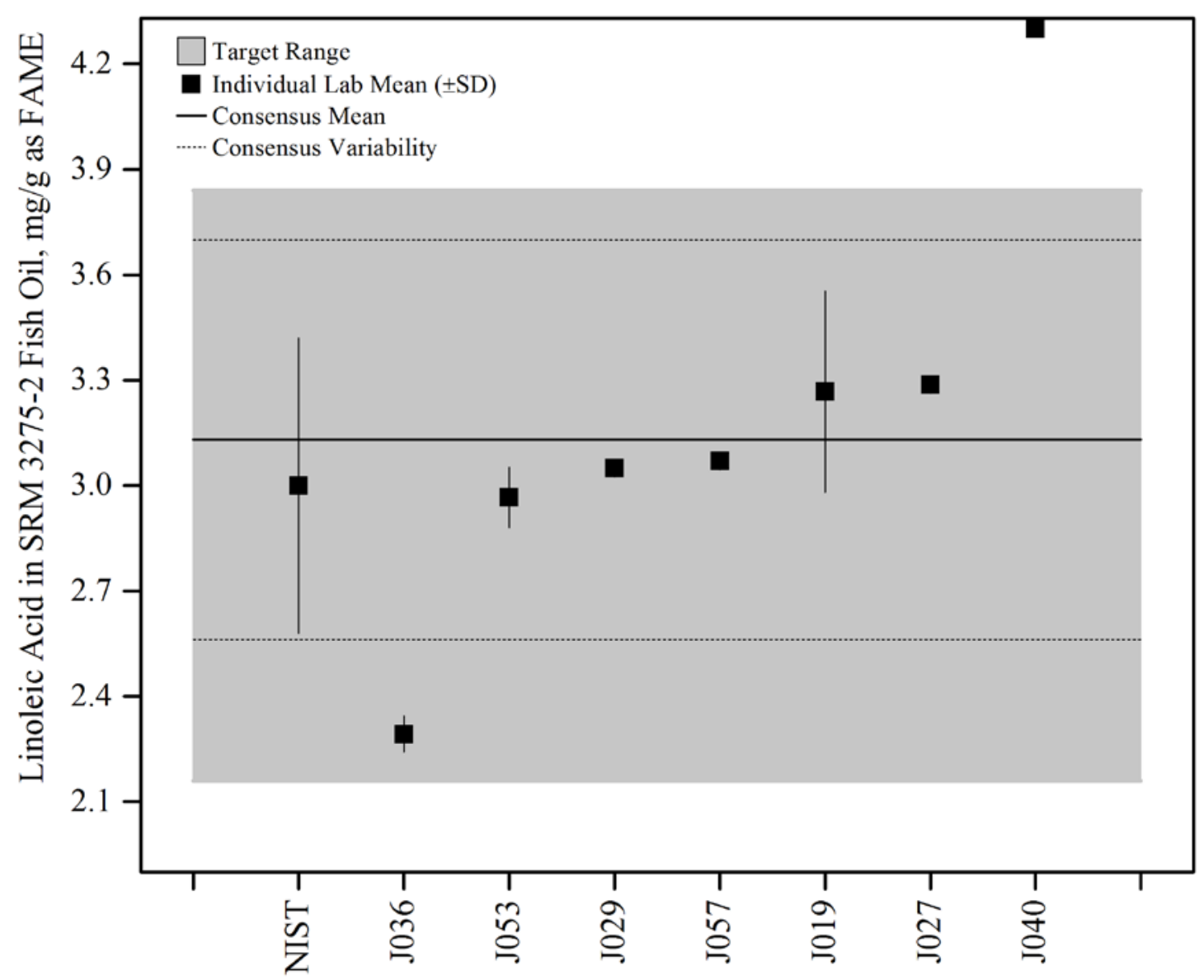

Figure 29. Linoleic acid in SRM 3275-2 Fish Oil (data summary view). In this view, individual laboratory data are plotted with the individual laboratory standard deviation (error bars). The black solid line represents the consensus mean, and the black dotted lines represent the consensus variability calculated as one standard deviation about the consensus mean. The gray shaded region represents the target zone for "acceptable" performance, which encompasses the NIST certified value bounded by twice its uncertainty $\left(U_{95}\right)$. 


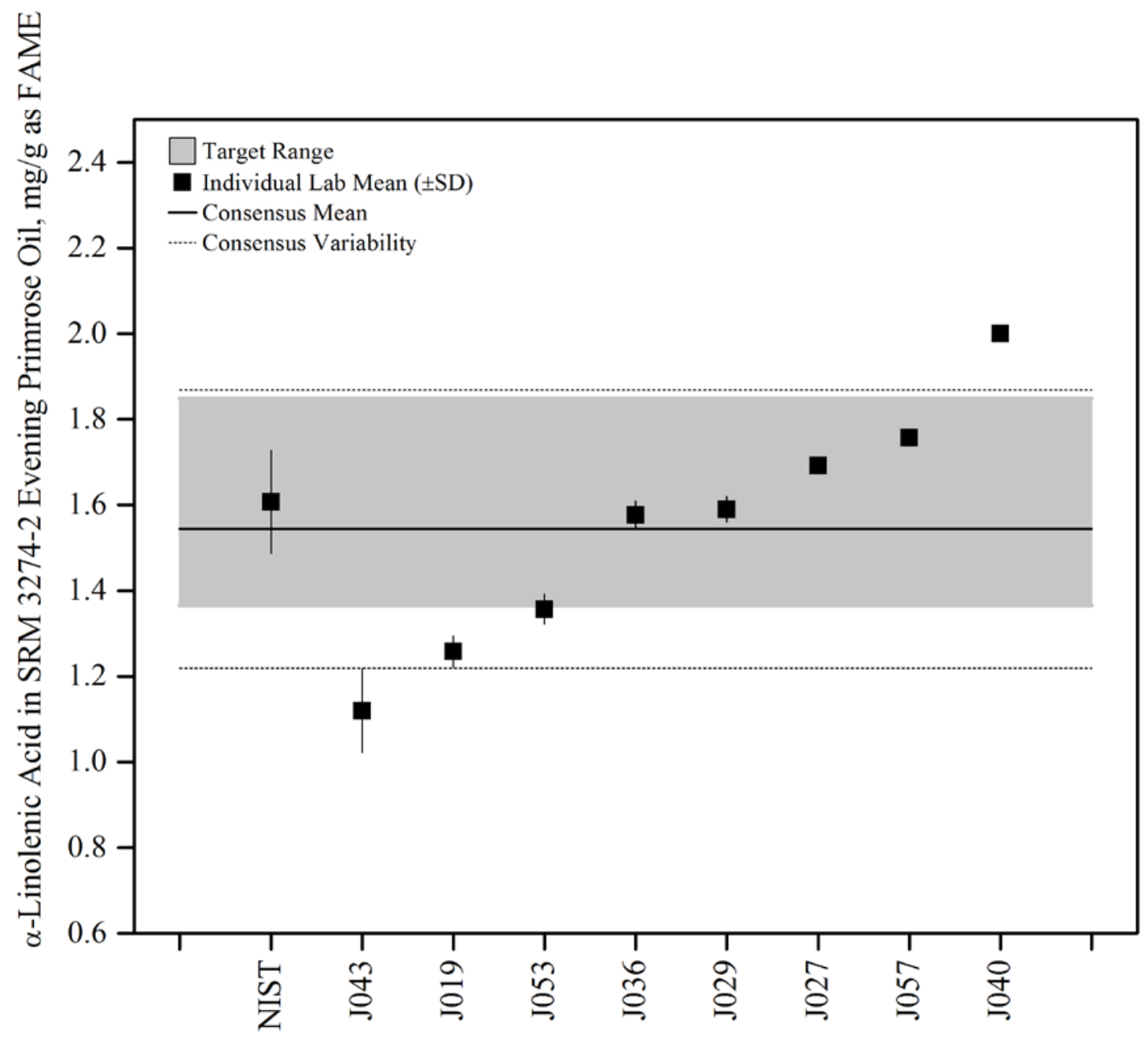

Figure 30. $\alpha$-Linolenic acid in SRM 3274-2 Evening Primrose Oil (data summary view). In this view, individual laboratory data are plotted with the individual laboratory standard deviation (error bars). The black solid line represents the consensus mean, and the black dotted lines represent the consensus variability calculated as one standard deviation about the consensus mean. The gray shaded region represents the target zone for "acceptable” performance, which encompasses the NIST reference value bounded by twice its uncertainty $\left(U_{95}\right)$. 


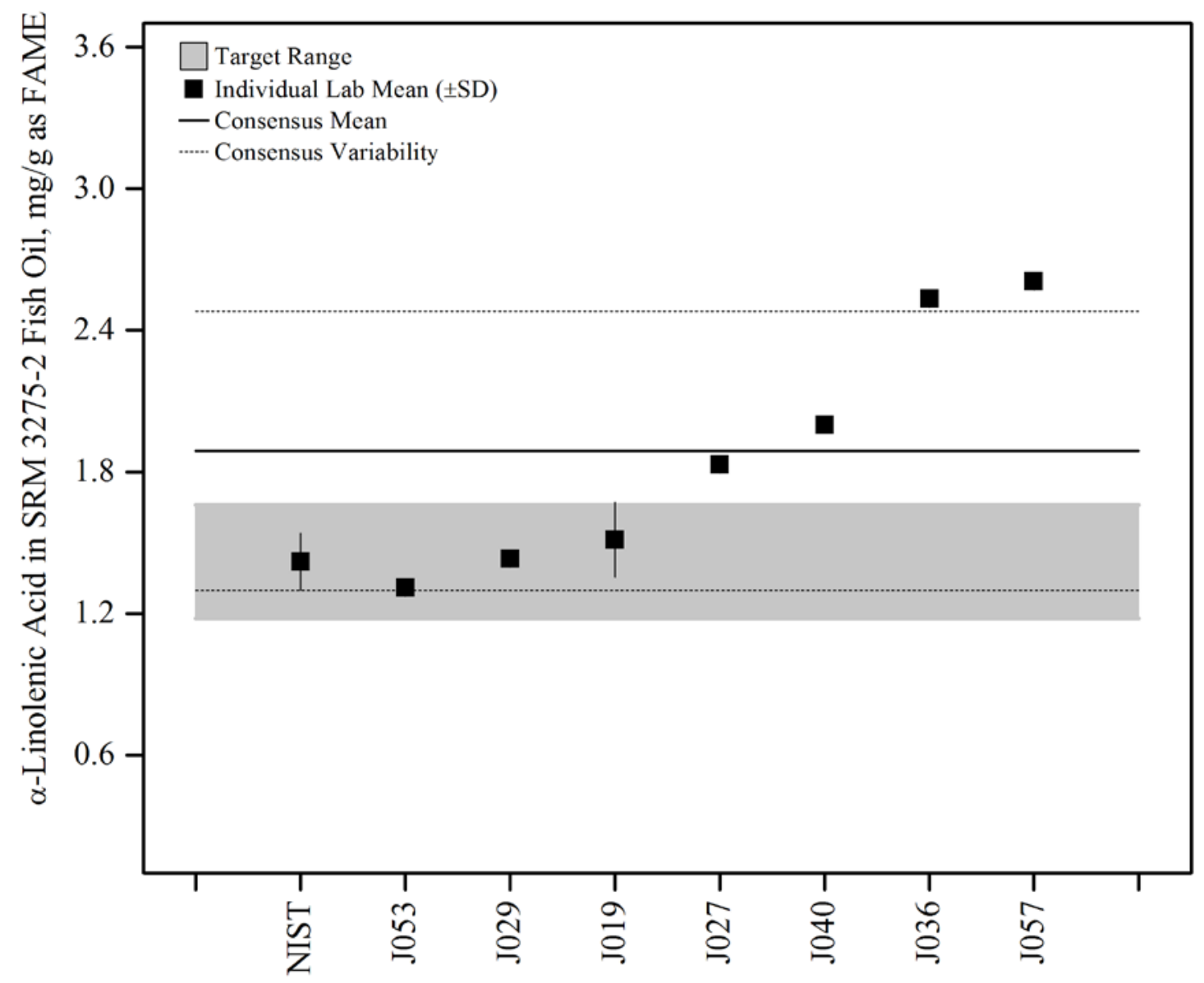

Figure 31. $\alpha$-Linolenic acid in SRM 3275-2 Fish Oil (data summary view). In this view, individual laboratory data are plotted with the individual laboratory standard deviation (error bars). The black solid line represents the consensus mean, and the black dotted lines represent the consensus variability calculated as one standard deviation about the consensus mean. The gray shaded region represents the target zone for "acceptable" performance, which encompasses the NIST reference value bounded by twice its uncertainty $\left(U_{95}\right)$. 


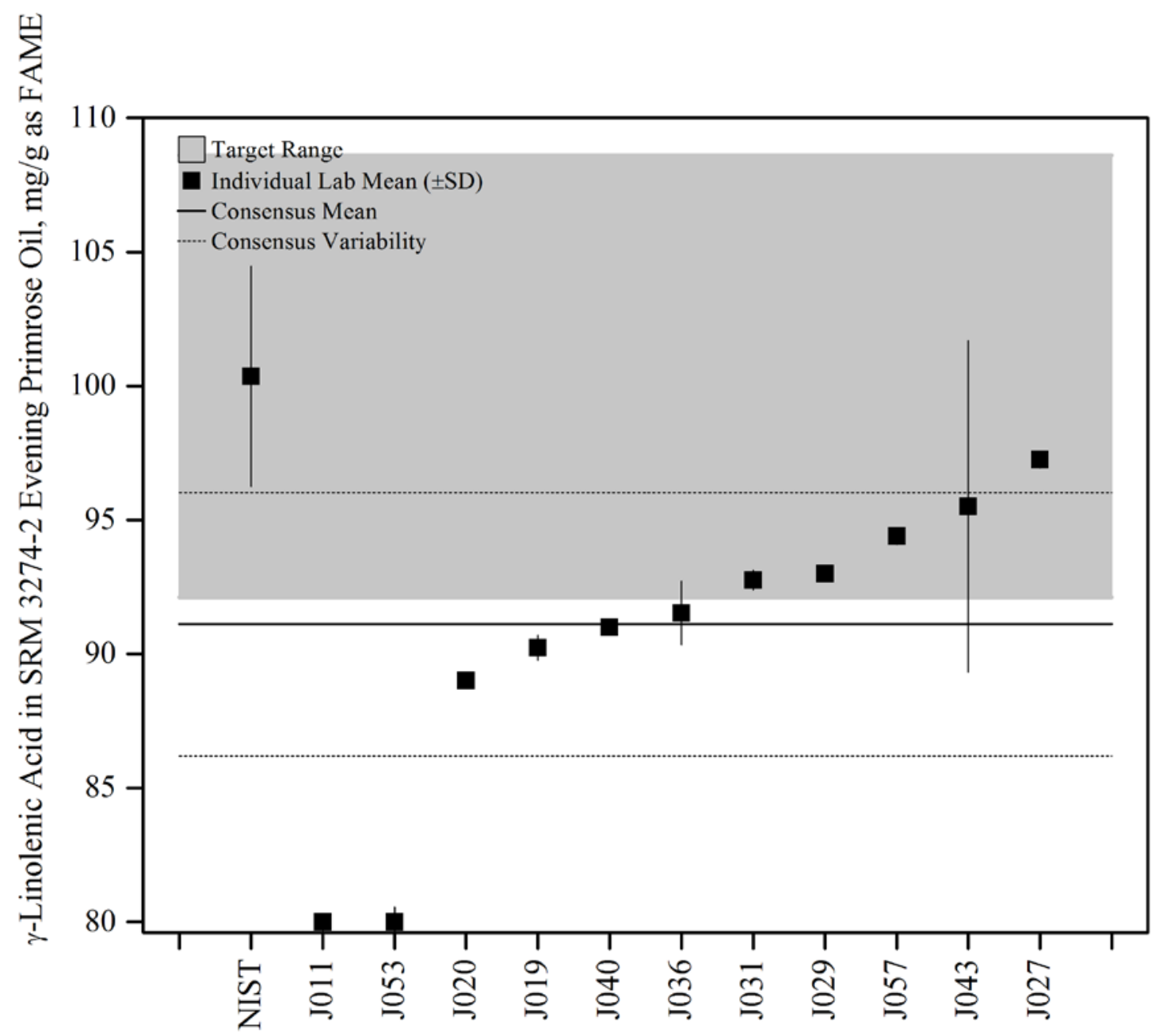

Figure 32. $\gamma$-Linolenic acid in SRM 3274-2 Evening Primrose Oil (data summary view). In this view, individual laboratory data are plotted with the individual laboratory standard deviation (error bars). The black solid line represents the consensus mean, and the black dotted lines represent the consensus variability calculated as one standard deviation about the consensus mean. The gray shaded region represents the target zone for "acceptable" performance, which encompasses the NIST certified value bounded by twice its uncertainty $\left(U_{95}\right)$. 


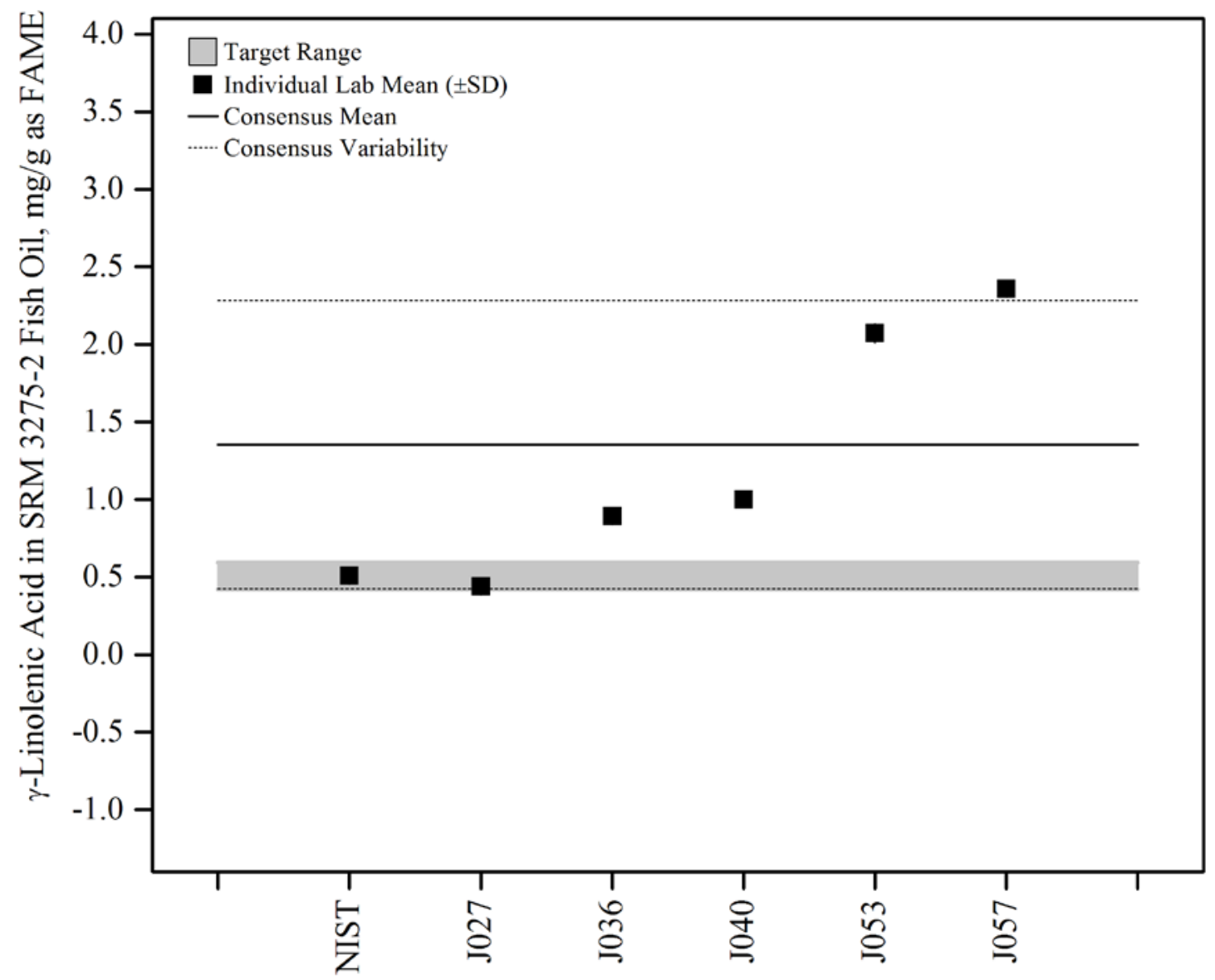

Figure 33. $\gamma$-Linolenic acid in SRM 3275-2 Fish Oil (data summary view). In this view, individual laboratory data are plotted with the individual laboratory standard deviation (error bars). The black solid line represents the consensus mean, and the black dotted lines represent the consensus variability calculated as one standard deviation about the consensus mean. The gray shaded region represents the target zone for "acceptable” performance, which encompasses the NIST reference value bounded by twice its uncertainty $\left(U_{95}\right)$. 


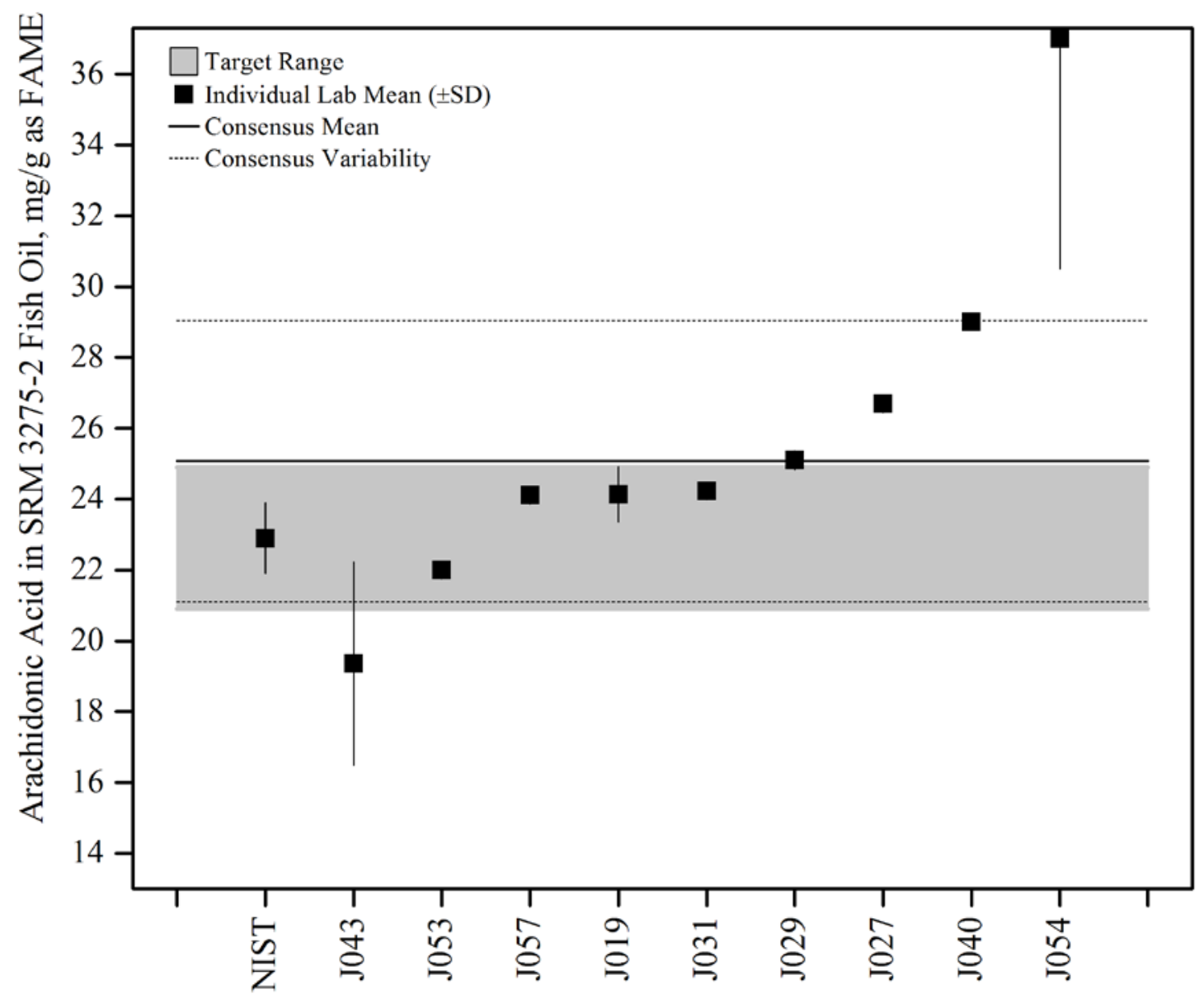

Figure 34. Arachidonic acid in SRM 3275-2 Fish Oil (data summary view). In this view, individual laboratory data are plotted with the individual laboratory standard deviation (error bars). The black solid line represents the consensus mean, and the black dotted lines represent the consensus variability calculated as one standard deviation about the consensus mean. The gray shaded region represents the target zone for "acceptable” performance, which encompasses the NIST reference value bounded by twice its uncertainty $\left(U_{95}\right)$. 


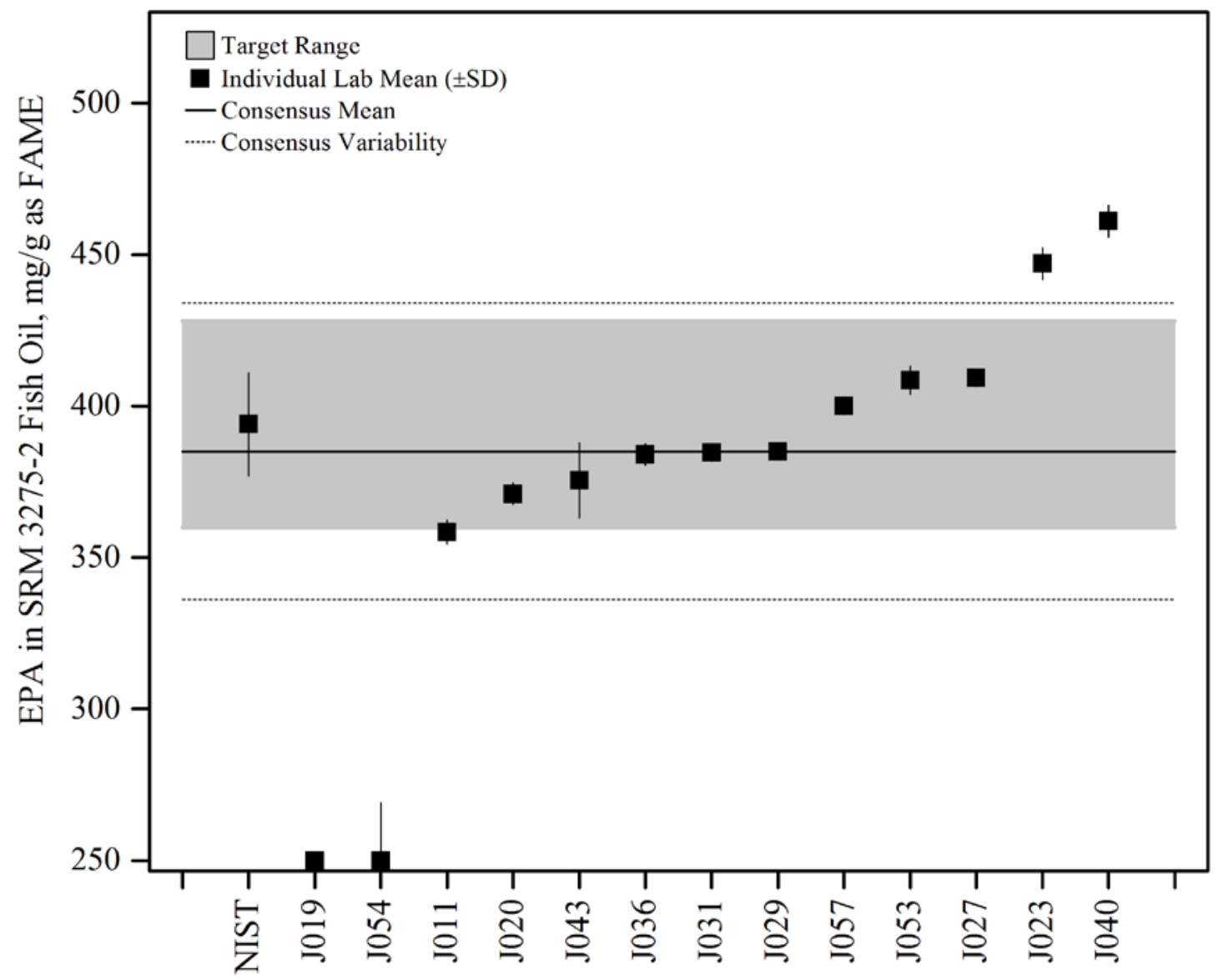

Figure 35. EPA in SRM 3275-2 Fish Oil (data summary view). In this view, individual laboratory data are plotted with the individual laboratory standard deviation (error bars). The black solid line represents the consensus mean, and the black dotted lines represent the consensus variability calculated as one standard deviation about the consensus mean. The gray shaded region represents the target zone for "acceptable" performance, which encompasses the NIST certified value bounded by twice its uncertainty $\left(U_{95}\right)$. 


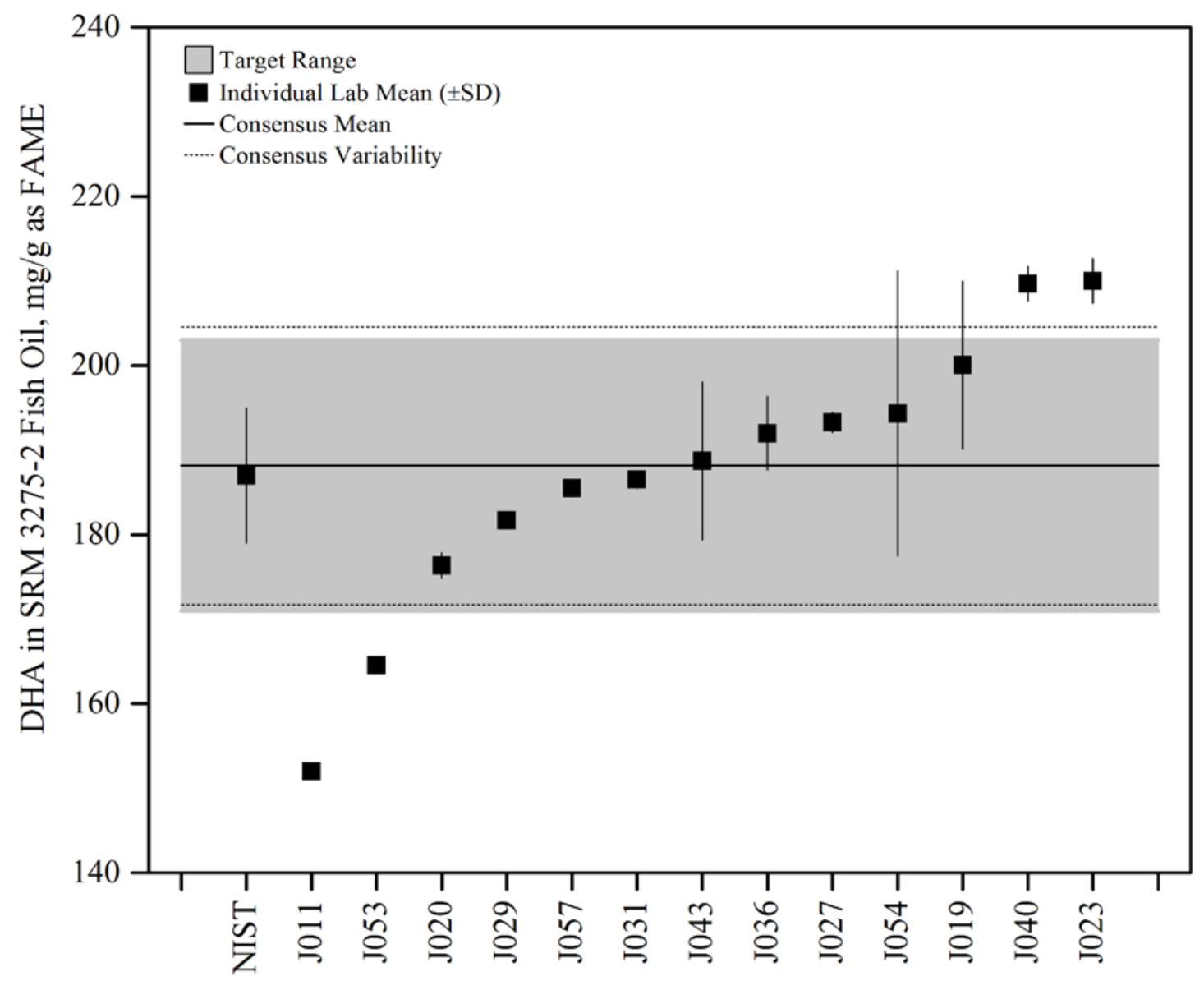

Figure 36. DHA in SRM 3275-2 Fish Oil (data summary view). In this view, individual laboratory data are plotted with the individual laboratory standard deviation (error bars). The black solid line represents the consensus mean, and the black dotted lines represent the consensus variability calculated as one standard deviation about the consensus mean. The gray shaded region represents the target zone for "acceptable" performance, which encompasses the NIST reference value bounded by twice its uncertainty $\left(U_{95}\right)$. 


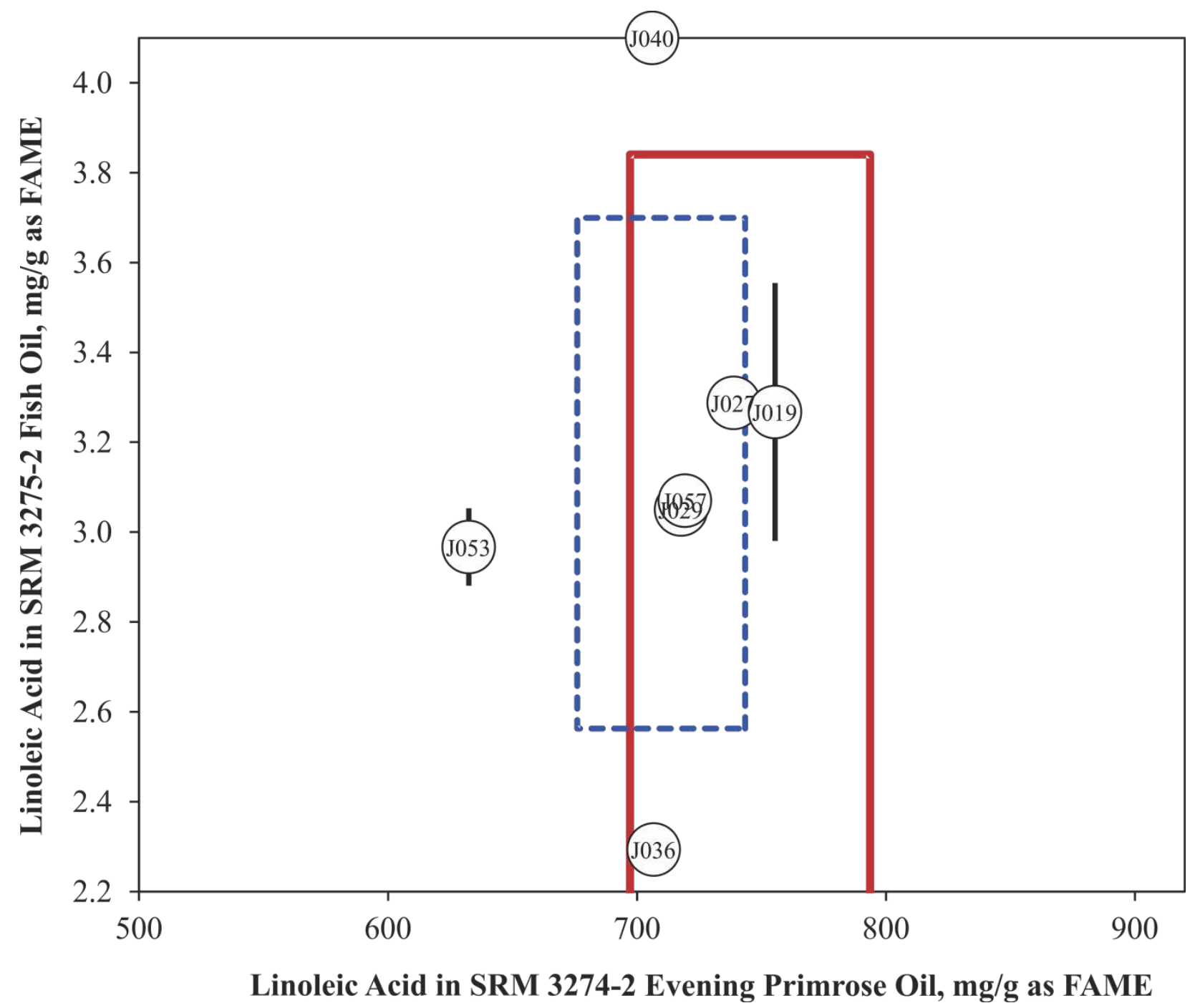

Figure 37. Linoleic acid in SRM 3274-2 Evening Primrose Oil and SRM 3275-2 Fish Oil-2 (sample/control comparison view). In this view, the individual laboratory results for the control (botanical oil) with a certified value for the analyte are compared to the results for an unknown (fish oil). The solid red box represents the target zone for the control (x-axis) and unknown sample (y-axis). The dotted blue box represents the consensus zone for the control (x-axis) and the unknown sample (y-axis). 


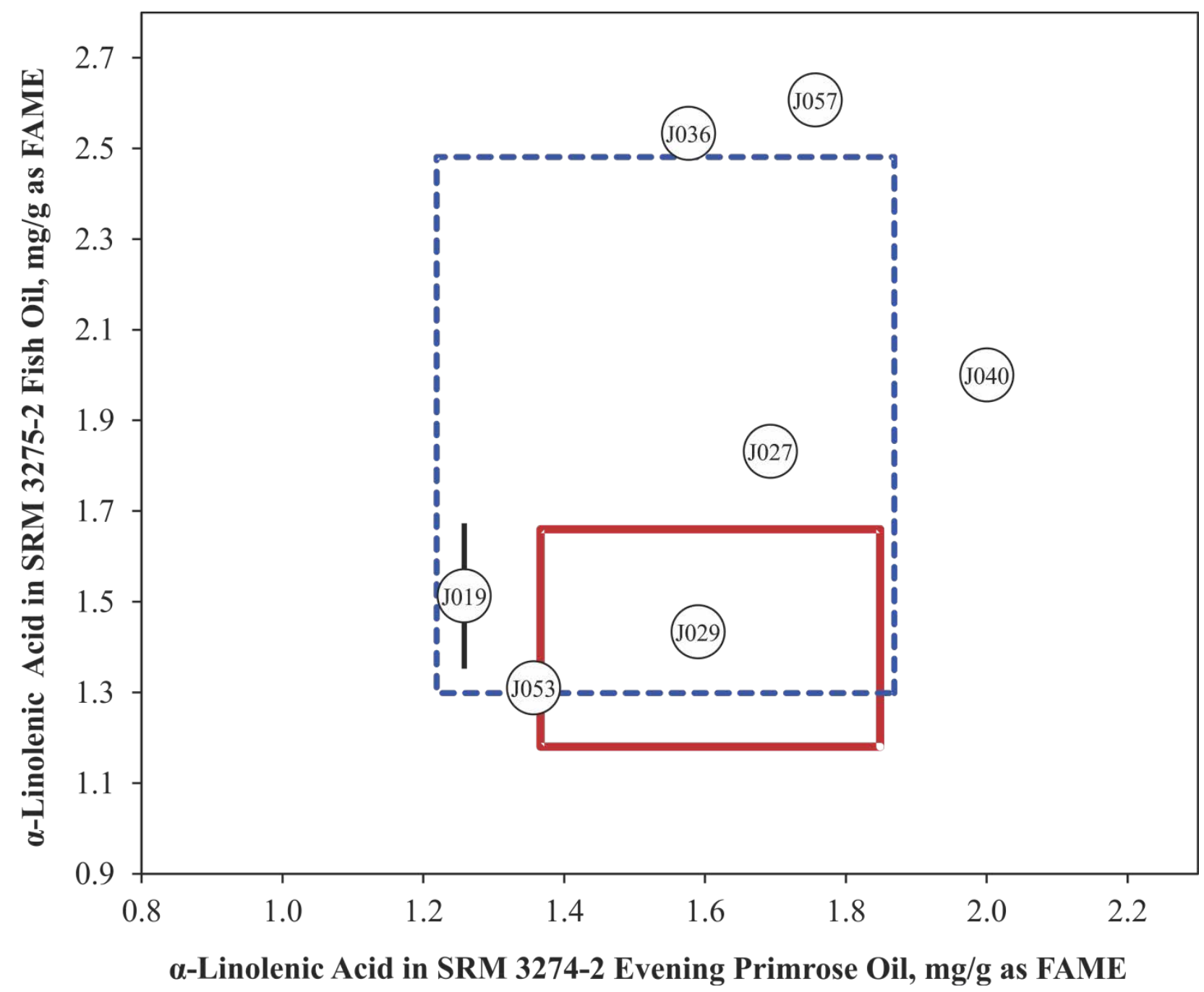

Figure 38. $\alpha$-Linolenic acid in SRM 3274-2 Evening Primrose Oil and SRM 3275-2 Fish Oil-2 (sample/control comparison view). In this view, the individual laboratory results for the control (botanical oil) with a certified value for the analyte are compared to the results for an unknown (fish oil). The solid red box represents the target zone for the control (x-axis) and unknown sample (y-axis). The dotted blue box represents the consensus zone for the control (x-axis) and the unknown sample (y-axis). 


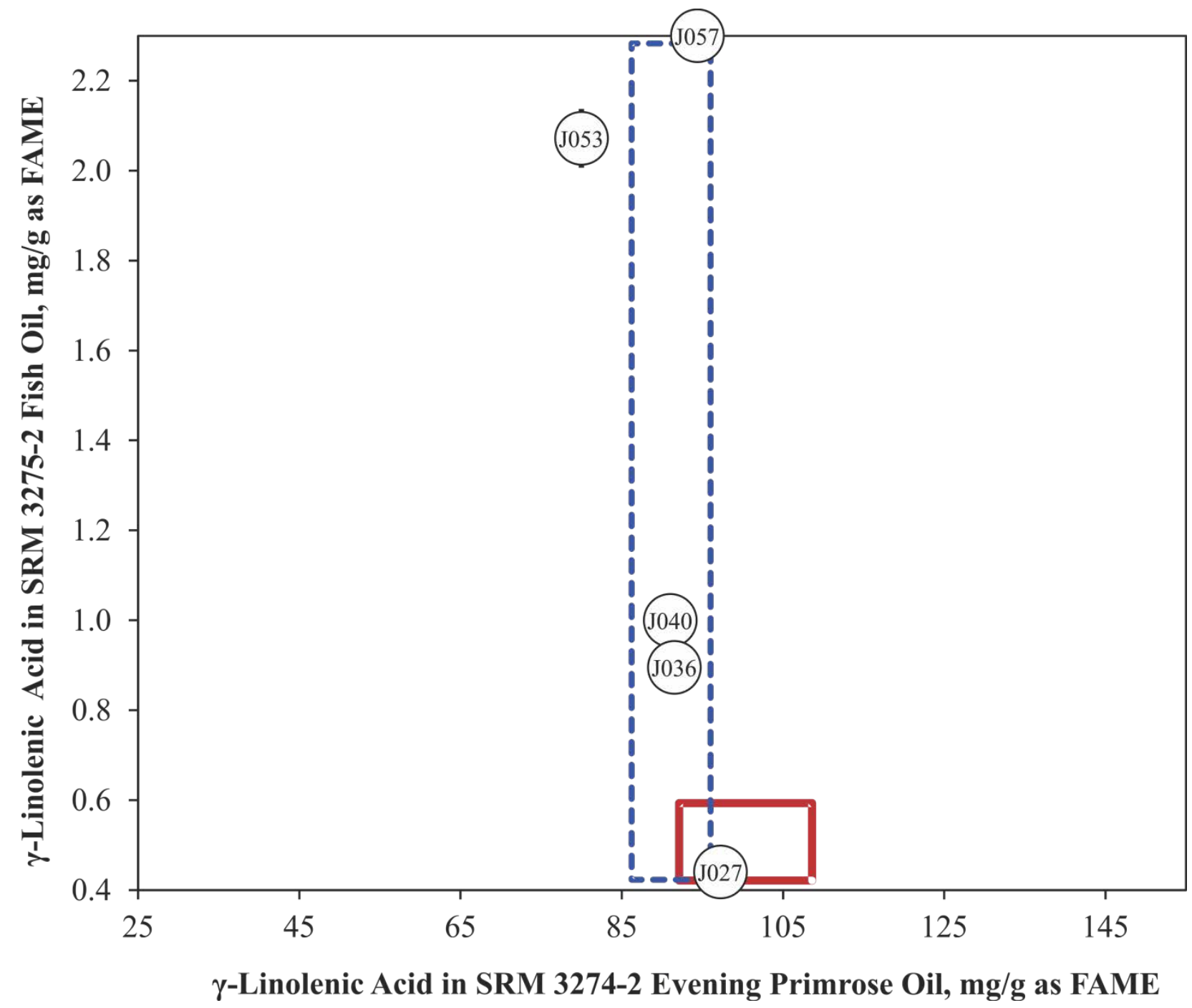

Figure 39. $\gamma$-Linolenic acid in SRM 3274-2 Evening Primrose Oil and SRM 3275-2 Fish Oil-2 (sample/control comparison view). In this view, the individual laboratory results for the control (botanical oil) with a certified value for the analyte are compared to the results for an unknown (fish oil). The solid red box represents the target zone for the control (x-axis) and unknown sample (y-axis). The dotted blue box represents the consensus zone for the control (x-axis) and the unknown sample (y-axis). 


\section{AFLATOXINS IN PEANUT BUTTER}

\section{Study Overview}

In this study, participants were provided with one NIST SRM, SRM 2387 Peanut Butter, and three peanut butter samples with low, medium, and high levels of aflatoxins. Participants were asked to use in-house analytical methods to determine the mass fractions of aflatoxins in each of the matrices and report values on an as-received basis. Participants were asked to report as many analytes as possible.

\section{Sample Information}

Peanut Butter. Participants were provided with one jar containing $170 \mathrm{~g}$ of peanut butter. Before use, participants were instructed to thoroughly mix the contents of the jar and a sample size of at least $50 \mathrm{~g}$ was recommended. Participants were asked to store the material under refrigeration, $0{ }^{\circ} \mathrm{C}$ to $4{ }^{\circ} \mathrm{C}$, and prepare three samples and report three values for as many analytes as possible from the single jar provided. Approximate analyte levels were not reported to participants prior to the study. NIST reference values and uncertainties, determined by collaborating laboratories, are outlined in the table below.

\section{Analyte \\ Aflatoxin B1 \\ Aflatoxin B2 \\ Total Aflatoxins}

\begin{tabular}{c} 
Reference Mass Fraction \\
in SRM 2387 (ng/g) \\
\hline $4.2 \pm 0.9$ \\
$0.7 \pm 0.3$ \\
$5.0 \pm 0.5$
\end{tabular}

Peanut Butter Samples. Participants were provided with three $50 \mathrm{~g}$ jars of peanut butter labeled 1,2 , and 3. Before use, participants were instructed to thoroughly mix the contents of each jar, and a sample size of at least $50 \mathrm{~g}$ was recommended. Participants were asked to store the material under refrigeration, $0{ }^{\circ} \mathrm{C}$ to $4{ }^{\circ} \mathrm{C}$, and prepare one sample and report one value for as many analytes as possible from each jar provided. Approximate analyte levels were not reported to participants prior to the study. NIST target values and uncertainties, determined by collaborating laboratories, are outlined in the table below.

\begin{tabular}{cccccc} 
& $\begin{array}{c}\text { Estimated Mass } \\
\text { Fraction in Peanut }\end{array}$ & $\begin{array}{c}\text { Estimated Mass } \\
\text { Fraction in Peanut }\end{array}$ & \multicolumn{2}{c}{$\begin{array}{c}\text { Estimated Mass } \\
\text { Fraction in Peanut }\end{array}$} \\
Analyte & $\begin{array}{c}\text { Butter 1 (Low) (ng/g) } \\
\text { Butter 2 (Med) (ng/g) }\end{array}$ & $\begin{array}{c}\text { Butter 3 (High) (ng/g) } \\
\text { Aflatoxin B1 }\end{array}$ & $3.70 \pm 0.63$ & $7.17 \pm 2.85$ & $10.95 \pm 2.22$ \\
Aflatoxin B2 & $1.01 \pm 0.09$ & $1.46 \pm 1.08$ & $2.16 \pm 0.49$ \\
Aflatoxin G1 & & & $0.68 \pm 0.29$ & $1.81 \pm 0.49$ \\
Aflatoxin G2 & & & $0.44 \pm 0.62$ & $0.43 \pm$ & 0.25 \\
Total Aflatoxins & $5.23 \pm 2.22$ & $9.95 \pm 1.43$ & $14.77 \pm$ & 2.85
\end{tabular}

Study Results

- Ten laboratories enrolled in this exercise and received samples. Five laboratories reported results for at least one aflatoxin per sample (50 \% participation).

- The consensus means for aflatoxins B1 and B2 and total aflatoxins were within the target range with high variability (26\% to $36 \%$ relative standard deviation (RSD)). 
- In the low-level sample, consensus means for aflatoxin B1 and total aflatoxins were within the target range, while the consensus mean for aflatoxin B2 was slightly below the target range. All three analytes were measured with high variability (27 \% to $38 \%$ RSD).

- In the mid-level sample, consensus means for all analytes were within the target ranges, but with high variability (14\% to $31 \%$ RSD).

- In the high-level sample, consensus means for all analytes were above the target ranges with high variability (33 \% to $49 \%$ RSD).

- Four of five laboratories reported using extraction for sample preparation (80\%). One laboratory reported using slurry blending sample preparation (20\%).

- Two laboratories reported using LC-fluorescence as their analytical method for analysis (40 \%). Three laboratories reported using LC/MS/MS to measure aflatoxins (60\%).

\section{Technical Recommendations}

The following recommendations are based on results obtained by the participants in this study.

- Because the data for this study was very limited (only 4 or 5 laboratories reporting data), drawing extensive technical conclusions is difficult. Similarly, the high level of betweenlaboratory variability may be exaggerated as a result of the low number of participants.

- No trends were identified indicating that a particular sample preparation method or instrumental technique provided more accurate results than another.

- Always check that the levels of the analytes in the samples are within the calibration range. The high-biased results for the high-level sample may result from extrapolation of the calibration curves beyond their linear ranges. 
Table 19. Individual data summary table (NIST) for aflatoxins in peanut butter.

\section{National Institute of Standards \& Technology}

\begin{tabular}{ccc} 
& & \\
& Lab Code: & NIST \\
\hline Analyte & Sample & Units \\
\hline Aflatoxin B1 & PB Control & $\mathrm{ng} / \mathrm{g}$ \\
Aflatoxin B1 & PB Sample 1 & $\mathrm{ng} / \mathrm{g}$ \\
Aflatoxin B1 & PB Sample 2 & $\mathrm{ng} / \mathrm{g}$ \\
Aflatoxin B1 & PB Sample 3 & $\mathrm{ng} / \mathrm{g}$ \\
\hline Aflatoxin B2 & PB Control & $\mathrm{ng} / \mathrm{g}$ \\
Aflatoxin B2 & PB Sample 1 & $\mathrm{ng} / \mathrm{g}$ \\
Aflatoxin B2 & PB Sample 2 & $\mathrm{ng} / \mathrm{g}$ \\
Aflatoxin B2 & PB Sample 3 & $\mathrm{ng} / \mathrm{g}$ \\
\hline Aflatoxin G1 & PB Control & $\mathrm{ng} / \mathrm{g}$ \\
Aflatoxin G1 & PB Sample 1 & $\mathrm{ng} / \mathrm{g}$ \\
Aflatoxin G1 & PB Sample 2 & $\mathrm{ng} / \mathrm{g}$ \\
Aflatoxin G1 & PB Sample 3 & $\mathrm{ng} / \mathrm{g}$ \\
\hline Aflatoxin G2 & PB Control & $\mathrm{ng} / \mathrm{g}$ \\
Aflatoxin G2 & PB Sample 1 & $\mathrm{ng} / \mathrm{g}$ \\
Aflatoxin G2 & PB Sample 2 & $\mathrm{ng} / \mathrm{g}$ \\
Aflatoxin G2 & PB Sample 3 & $\mathrm{ng} / \mathrm{g}$ \\
\hline Total Aflatoxins & PB Control & $\mathrm{ng} / \mathrm{g}$ \\
Total Aflatoxins & PB Sample 1 & $\mathrm{ng} / \mathrm{g}$ \\
Total Aflatoxins & PB Sample 2 & $\mathrm{ng} / \mathrm{g}$ \\
Total Aflatoxins & PB Sample 3 & $\mathrm{ng} / \mathrm{g}$ \\
\hline
\end{tabular}

Exercise J - May 2013 - Aflatoxins

\begin{tabular}{cccc}
\multicolumn{4}{c}{ 1. Your Results } \\
\hline $\mathrm{x}_{\mathrm{i}}$ & $\mathrm{s}_{\mathrm{i}}$ & $\mathrm{Z}_{\text {comm }}$ & $\mathrm{Z}_{\text {NIST }}$ \\
\hline 4.2 & 0.9 & -0.1 & 0.0 \\
3.70 & 0.63 & 0.4 & 0.0 \\
7.17 & 3 & -0.7 & 0.0 \\
11.00 & 2.22 & -0.6 & 0.0 \\
\hline 0.7 & 0.3 & -0.1 & 0.0 \\
1.01 & 0.09 & 0.4 & 0.0 \\
1.46 & 1.08 & -0.4 & 0.0 \\
2.16 & 0.49 & -0.8 & 0.0 \\
\hline
\end{tabular}

\begin{tabular}{crc}
\multicolumn{3}{c}{ 2. Community Results } \\
\hline $\mathrm{N}$ & $\mathrm{x}^{*}$ & $\mathrm{~s}^{*}$ \\
\hline
\end{tabular}

\begin{tabular}{cc}
\multicolumn{2}{c}{ 3. Target } \\
\hline $\mathrm{x}_{\mathrm{NIST}}$ & $U_{95}$ \\
\hline 4.2 & 0.9 \\
3.70 & 0.63 \\
7.17 & 2.85 \\
11.0 & 2.2 \\
\hline 0.7 & 0.3 \\
1.01 & 0.09 \\
1.46 & 1.08 \\
2.16 & 0.49 \\
\hline
\end{tabular}

\begin{tabular}{llll}
0.68 & 0.29 & -0.8 & 0.0 \\
1.81 & 0.49 & -0.7 & 0.0 \\
\hline
\end{tabular}

\begin{tabular}{ccc}
\hline 4 & 4.4 & 1.6 \\
4 & 3.36 & 0.96
\end{tabular}

\begin{tabular}{ccc}
4 & 8.62 & 2.10 \\
4 & 13.9 & 4.5 \\
\hline
\end{tabular}

\begin{tabular}{ccc}
4 & 13.9 & 4.5 \\
\hline 4 & 0.7 & 0.2 \\
4 & 0.88 & 0.33
\end{tabular}

\begin{tabular}{rrr}
4 & 1.69 & 0.53 \\
4 & 3.59 & 1.77 \\
\hline
\end{tabular}

\begin{tabular}{ll}
2.16 & 0.49 \\
\hline
\end{tabular}

\begin{tabular}{lll}
3 & 0.77 & 0.11 \\
3 & 2.29 & 0.66 \\
\hline 0 &
\end{tabular}

\begin{tabular}{ll}
0.68 & 0.29 \\
1.81 & 0.49 \\
\hline
\end{tabular}

\begin{tabular}{cccc}
0.44 & 0.62 & & 0.0 \\
0.43 & 0.25 & $<-4$ & 0.0 \\
\hline 5.0 & 0.5 & -0.1 & 0.0 \\
5.23 & 2.22 & 0.9 & 0.0 \\
9.95 & 1.43 & -0.5 & 0.0 \\
14.80 & 2.85 & -0.5 & 0.0 \\
\hline
\end{tabular}

\begin{tabular}{ccc}
0 & & \\
0 & & \\
2 & 0.52 & 0.00 \\
\hline 5 & 5.2 & 1.4 \\
5 & 4.20 & 1.11 \\
5 & 10.90 & 2.02 \\
5 & 18.0 & 6.2 \\
\hline
\end{tabular}

\begin{tabular}{cc}
0.44 & 0.62 \\
0.43 & 0.25 \\
\hline 5.0 & 0.5 \\
5.23 & 2.22 \\
9.95 & 1.43 \\
14.8 & 2.9 \\
\hline
\end{tabular}

$x_{i}$ Mean of reported values

$\mathrm{s}_{\mathrm{i}}$ Standard deviation of reported values

$\mathrm{Z}_{\text {comm }}$ Z-score with respect to community consensus

$Z_{\text {NIST }}$ Z-score with respect to NIST value
$\mathrm{N}$ Number of quantitative values reported

$\mathrm{x}^{*}$ Robust mean of reported values

s* Robust standard deviation $\mathrm{x}_{\text {NIST }}$ NIST-assessed value

$U_{95} \pm 95 \%$ confidence interval about the assessed value or standard deviation $\left(\mathrm{s}_{\mathrm{NIST}}\right.$ ) 
Table 20. Data summary table for aflatoxin B1 in peanut butter.

\begin{tabular}{|c|c|c|c|c|c|c|c|c|c|}
\hline & \multirow[b]{3}{*}{ Lab } & \multicolumn{8}{|c|}{ Aflatoxin B1 } \\
\hline & & \multicolumn{5}{|c|}{ SRM 2387 Peanut Butter (ng/g) } & \multicolumn{3}{|c|}{ Peanut Butter Samples (ng/g) } \\
\hline & & A & B & $\mathbf{C}$ & Avg & SD & Lo & Med & $\mathbf{H i}$ \\
\hline \multirow{11}{*}{ 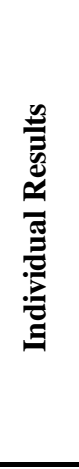 } & NIST & & & & 4.2 & 0.9 & $3.70 \pm 0.63$ & $7.17 \pm 2.85$ & $11.0 \pm 2.2$ \\
\hline & J002 & & & & & & & & \\
\hline & J007 & & & & & & & & \\
\hline & J008 & & & & & & & & \\
\hline & J029 & 5.4 & 5.4 & 5.8 & 5.5 & 0.2 & 2.60 & 7.85 & 13.2 \\
\hline & $\mathrm{J} 030$ & 2.2 & 2.0 & 3.6 & 2.6 & 0.9 & 2.67 & 7.04 & 9.6 \\
\hline & J035 & & & & & & & & \\
\hline & J036 & & & & & & & & \\
\hline & J052 & 3.9 & 4.0 & 3.8 & 3.9 & 0.1 & 3.90 & 8.30 & 13.4 \\
\hline & J053 & 5.4 & 5.6 & 5.3 & 5.4 & 0.2 & 4.25 & 11.29 & 19.2 \\
\hline & J059 & & & & & & & & \\
\hline \multirow{5}{*}{ 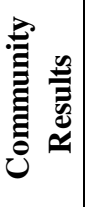 } & & Consen & & & 4.4 & & 3.36 & 8.62 & 13.9 \\
\hline & & Consen & dard & & 1.6 & & 0.96 & 2.10 & 4.5 \\
\hline & & Maximı & & & 5.5 & & 4.25 & 11.29 & 19.2 \\
\hline & & Minimu & & & 2.6 & & 2.60 & 7.04 & 9.6 \\
\hline & & $\mathrm{N}$ & & & 4 & & 4 & 4 & 4 \\
\hline
\end{tabular}

Table 21. Data summary table for aflatoxin B2 in peanut butter.

\begin{tabular}{|c|c|c|c|c|c|c|c|c|c|}
\hline & \multirow[b]{3}{*}{ Lab } & \multicolumn{8}{|c|}{ Aflatoxin B2 } \\
\hline & & \multicolumn{5}{|c|}{ SRM 2387 Peanut Butter (ng/g) } & \multicolumn{3}{|c|}{ Peanut Butter Samples (ng/g) } \\
\hline & & A & B & $\mathbf{C}$ & Avg & SD & Lo & Med & Hi \\
\hline \multirow{11}{*}{ 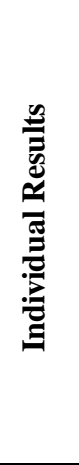 } & NIST & & & & 0.7 & 0.3 & $1.01 \pm 0.09$ & $1.46 \pm 1.08$ & $2.16 \pm 0.49$ \\
\hline & J002 & & & & & & & & \\
\hline & J007 & & & & & & & & \\
\hline & J008 & & & & & & & & \\
\hline & J029 & 0.9 & 0.9 & 1.0 & 1.0 & 0.0 & 0.70 & 1.18 & 2.48 \\
\hline & J030 & 0.5 & 0.7 & 0.8 & 0.6 & 0.2 & 0.96 & 1.58 & 2.33 \\
\hline & J035 & & & & & & & & \\
\hline & J036 & & & & & & & & \\
\hline & J052 & 0.6 & 0.5 & 0.6 & 0.6 & 0.1 & 0.60 & 2.30 & 5.70 \\
\hline & J053 & 0.8 & 0.8 & 0.6 & 0.7 & 0.1 & 1.25 & 1.68 & 3.86 \\
\hline & J059 & & & & & & & & \\
\hline \multirow{5}{*}{ 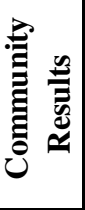 } & & Consen & & & 0.7 & & 0.88 & 1.69 & 3.59 \\
\hline & & Consen & ndard & & 0.2 & & 0.33 & 0.53 & 1.77 \\
\hline & & Maximı & & & 1.0 & & 1.25 & 2.30 & 5.70 \\
\hline & & Minimu & & & 0.6 & & 0.60 & 1.18 & 2.33 \\
\hline & & $\mathrm{N}$ & & & 4 & & 4 & 4 & 4 \\
\hline
\end{tabular}


Table 22. Data summary table for aflatoxin G1 in peanut butter.

\begin{tabular}{|c|c|c|c|c|c|c|c|c|c|}
\hline & \multirow[b]{3}{*}{ Lab } & \multicolumn{8}{|c|}{ Aflatoxin G1 } \\
\hline & & & $\mathrm{M}$ & anut & $r(n g)$ & & & Butter Sampl & (ng/g) \\
\hline & & A & B & C & Avg & SD & Lo & Med & $\mathrm{Hi}$ \\
\hline \multirow{11}{*}{ 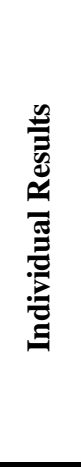 } & NIST & & & & & & & $0.68 \pm 0.29$ & $1.81 \pm 0.49$ \\
\hline & J002 & & & & & & & & \\
\hline & J007 & & & & & & & & \\
\hline & J008 & & & & & & & & \\
\hline & J029 & & & & & & & 0.66 & 2.14 \\
\hline & J030 & 0.3 & 0.3 & 0.3 & 0.3 & 0.0 & & 0.81 & 1.79 \\
\hline & J035 & & & & & & & & \\
\hline & J036 & & & & & & & & \\
\hline & J052 & & & & & & & & \\
\hline & J053 & & & & & & & 0.84 & 2.93 \\
\hline & J059 & & & & & & & & \\
\hline \multirow{5}{*}{ 疍 } & & \multirow{5}{*}{\multicolumn{3}{|c|}{$\begin{array}{l}\text { Consensus Mean } \\
\text { Consensus Standard Deviation } \\
\text { Maximum } \\
\text { Minimum } \\
\mathrm{N}\end{array}$}} & & & & 0.77 & 2.29 \\
\hline & & & & & & & & 0.11 & 0.66 \\
\hline & & & & & & & & 0.84 & 2.93 \\
\hline & & & & & & & & 0.66 & 1.79 \\
\hline & & & & & 1 & & 0 & 3 & 3 \\
\hline
\end{tabular}

Table 23. Data summary table for aflatoxin G2 in peanut butter.

\begin{tabular}{|c|c|c|c|c|c|c|c|c|c|}
\hline & \multirow[b]{3}{*}{ Lab } & \multicolumn{8}{|c|}{ Aflatoxin G2 } \\
\hline & & & M & anu & $r(n g)$ & & & utter Sampl & (ng/g) \\
\hline & & A & B & C & Avg & SD & Lo & Med & Hi \\
\hline \multirow{11}{*}{ 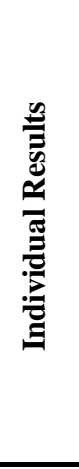 } & NIST & & & & & & & $0.44 \pm 0.62$ & $0.43 \pm 0.25$ \\
\hline & J002 & & & & & & & & \\
\hline & J007 & & & & & & & & \\
\hline & J008 & & & & & & & & \\
\hline & J029 & & & & & & & & 0.52 \\
\hline & J030 & & & & & & & & 0.52 \\
\hline & J035 & & & & & & & & \\
\hline & J036 & & & & & & & & \\
\hline & J052 & & & & & & & & \\
\hline & J053 & & & & & & & & \\
\hline & J059 & & & & & & & & \\
\hline \multirow{5}{*}{ 恿 } & & \multirow{5}{*}{\multicolumn{5}{|c|}{$\begin{array}{l}\text { Consensus Mean } \\
\text { Consensus Standard Deviation } \\
\text { Maximum } \\
\text { Minimum } \\
\text { N }\end{array}$}} & & & 0.52 \\
\hline & & & & & & & & & 0.00 \\
\hline & & & & & & & & & 0.52 \\
\hline & & & & & & & & & 0.52 \\
\hline & & & & & & & 0 & 0 & 2 \\
\hline
\end{tabular}


Table 24. Data summary table for total aflatoxins in peanut butter.

\begin{tabular}{|c|c|c|c|c|c|c|c|c|c|}
\hline & \multirow[b]{3}{*}{ Lab } & \multicolumn{8}{|c|}{ Total Aflatoxins } \\
\hline & & \multicolumn{5}{|c|}{ SRM 2387 Peanut Butter (ng/g) } & \multicolumn{3}{|c|}{ Peanut Butter Samples (ng/g) } \\
\hline & & A & B & C & Avg & SD & Lo & Med & $\mathrm{Hi}$ \\
\hline \multirow{11}{*}{ 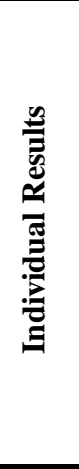 } & NIST & & & & 5.0 & 0.5 & $5.23 \pm 2.22$ & $9.95 \pm 1.43$ & $14.8 \pm 2.9$ \\
\hline & J002 & & & & & & & & \\
\hline & J007 & & & & & & & & \\
\hline & J008 & 5.5 & 6.0 & 4.3 & 5.3 & 0.9 & 3.80 & 11.00 & 12.0 \\
\hline & J029 & 6.4 & 6.3 & 6.8 & 6.5 & 0.3 & 3.30 & 9.69 & 18.3 \\
\hline & J030 & 2.9 & 3.0 & 4.7 & 3.5 & 1.0 & 3.63 & 9.43 & 14.3 \\
\hline & J035 & & & & & & & & \\
\hline & J036 & & & & & & & & \\
\hline & J052 & 4.5 & 4.5 & 4.4 & 4.5 & 0.1 & 4.50 & 10.60 & 19.1 \\
\hline & J053 & 6.2 & 6.4 & 5.9 & 6.2 & 0.3 & 5.77 & 13.89 & 26.3 \\
\hline & J059 & & & & & & & & \\
\hline \multirow{5}{*}{ 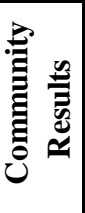 } & & Consen & & & 5.2 & & 4.20 & 10.92 & 18.0 \\
\hline & & Consen & dard & & 1.4 & & 1.11 & 2.02 & 6.2 \\
\hline & & Maximı & & & 6.5 & & 5.77 & 13.89 & 26.3 \\
\hline & & Minimu & & & 3.5 & & 3.30 & 9.43 & 12.0 \\
\hline & & $\mathrm{N}$ & & & 5 & & 5 & 5 & 5 \\
\hline
\end{tabular}




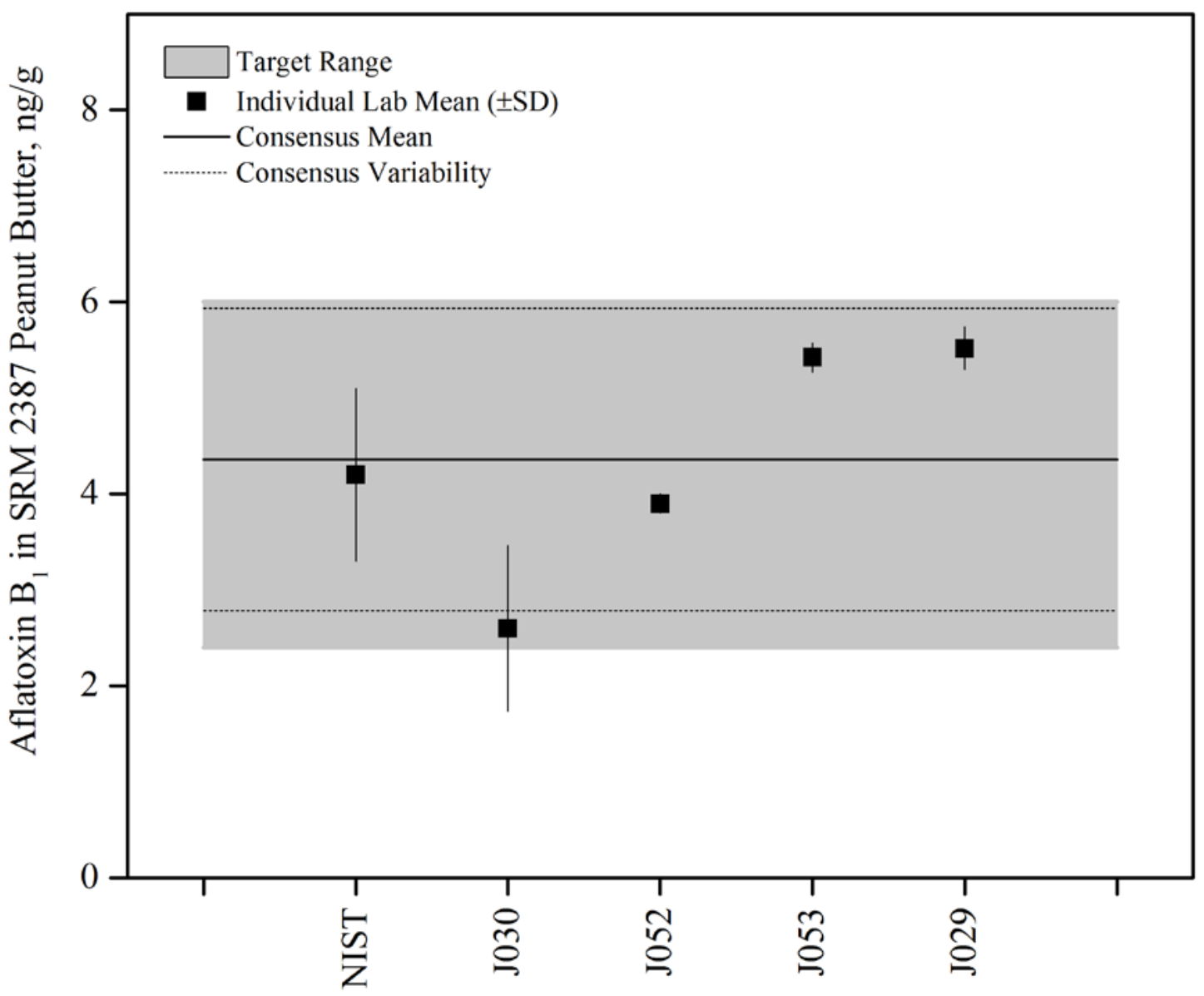

Figure 40. Aflatoxin B1 in SRM 2387 Peanut Butter (data summary view). In this view, individual laboratory data are plotted with the individual laboratory standard deviation (error bars). The black solid line represents the consensus mean, and the black dotted lines represent the consensus variability calculated as one standard deviation about the consensus mean. The gray shaded region represents the target zone for "acceptable" performance, which encompasses the NIST reference value bounded by twice its uncertainty $\left(U_{95}\right)$. 


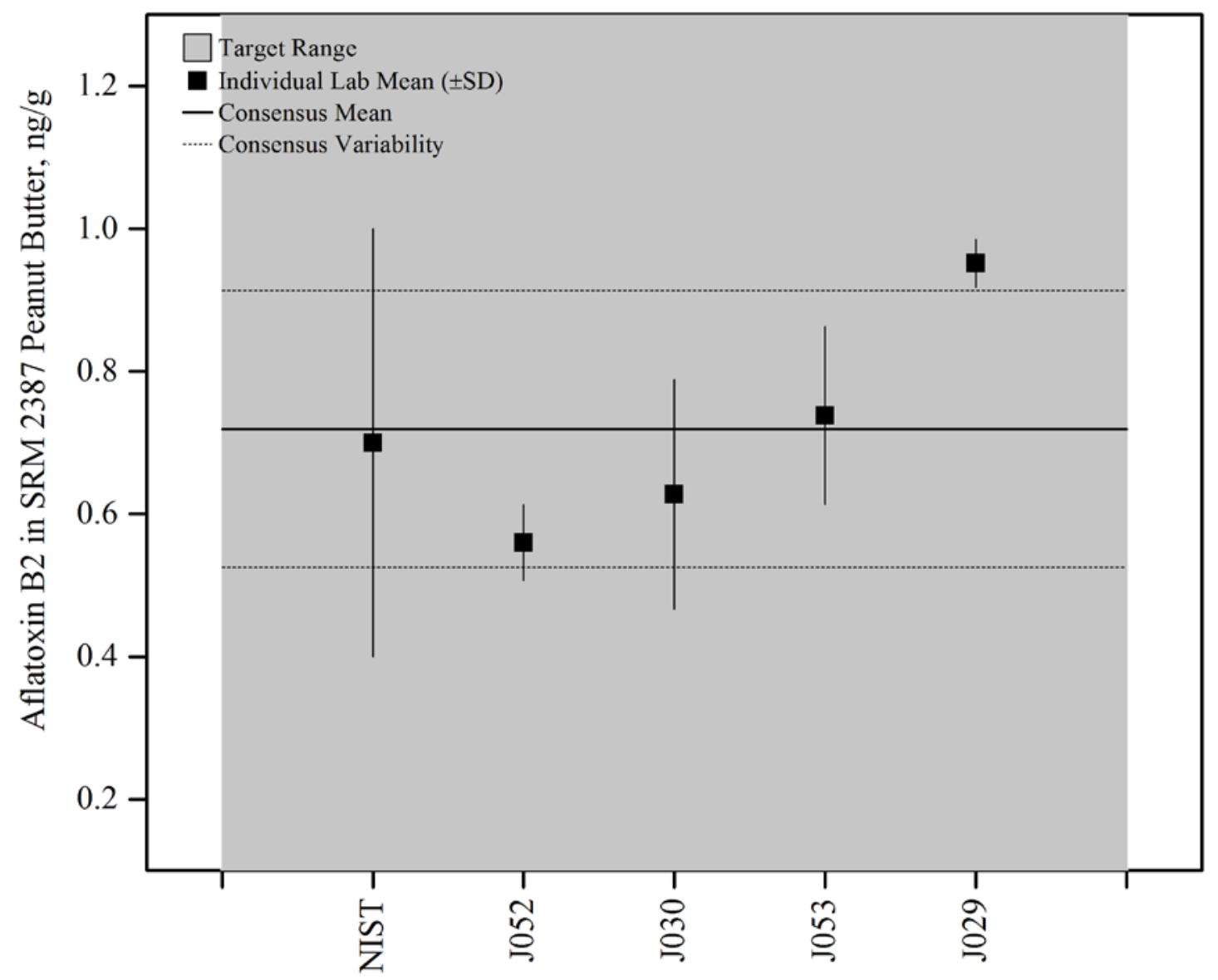

Figure 41. Aflatoxin B2 in SRM 2387 Peanut Butter (data summary view). In this view, individual laboratory data are plotted with the individual laboratory standard deviation (error bars). The black solid line represents the consensus mean, and the black dotted lines represent the consensus variability calculated as one standard deviation about the consensus mean. The gray shaded region represents the target zone for "acceptable” performance, which encompasses the NIST reference value bounded by twice its uncertainty $\left(U_{95}\right)$. 


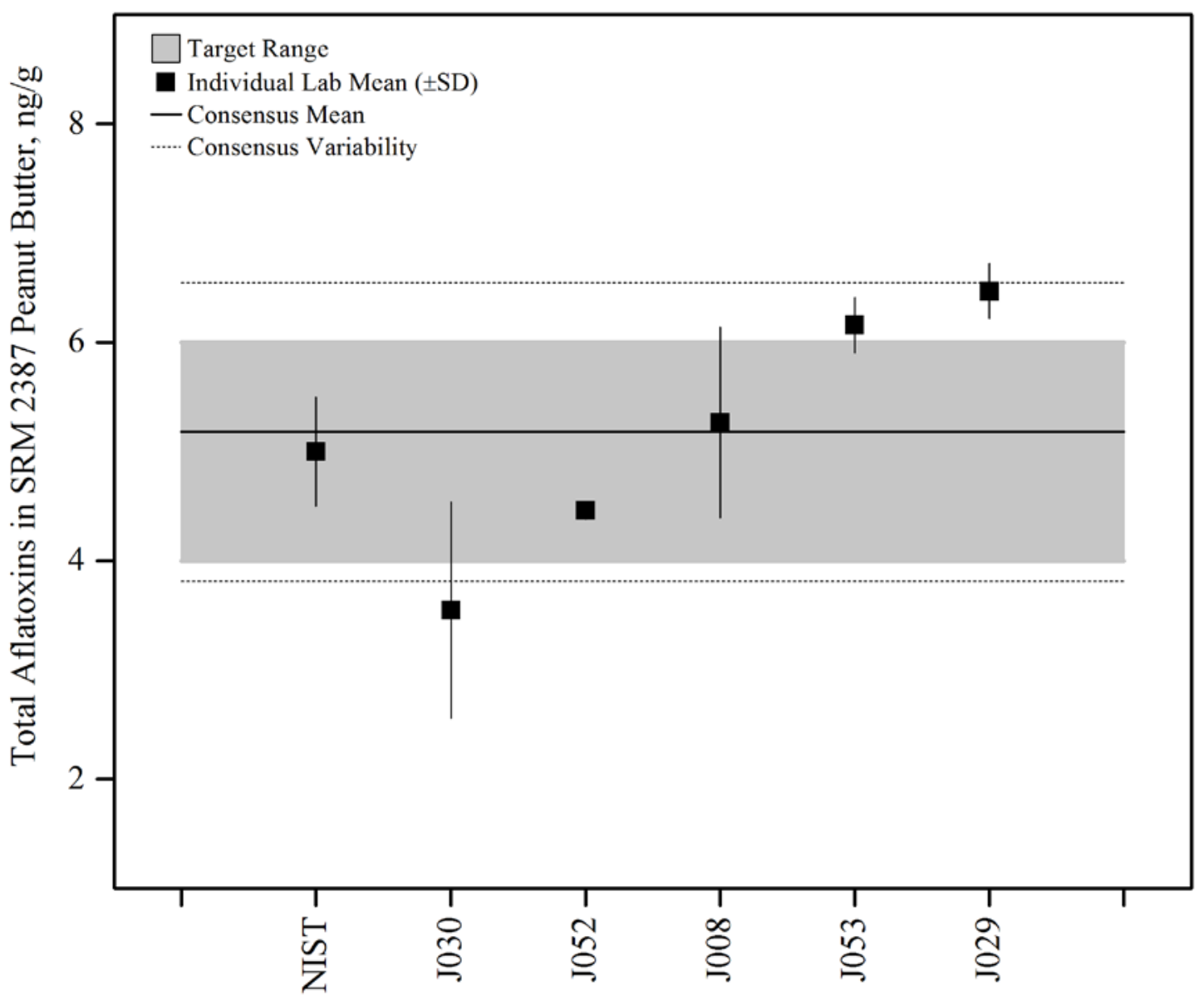

Figure 42. Total aflatoxins in SRM 2387 Peanut Butter (data summary view). In this view, individual laboratory data are plotted with the individual laboratory standard deviation (error bars). The black solid line represents the consensus mean, and the black dotted lines represent the consensus variability calculated as one standard deviation about the consensus mean. The gray shaded region represents the target zone for "acceptable” performance, which encompasses the NIST reference value bounded by twice its uncertainty $\left(U_{95}\right)$. 


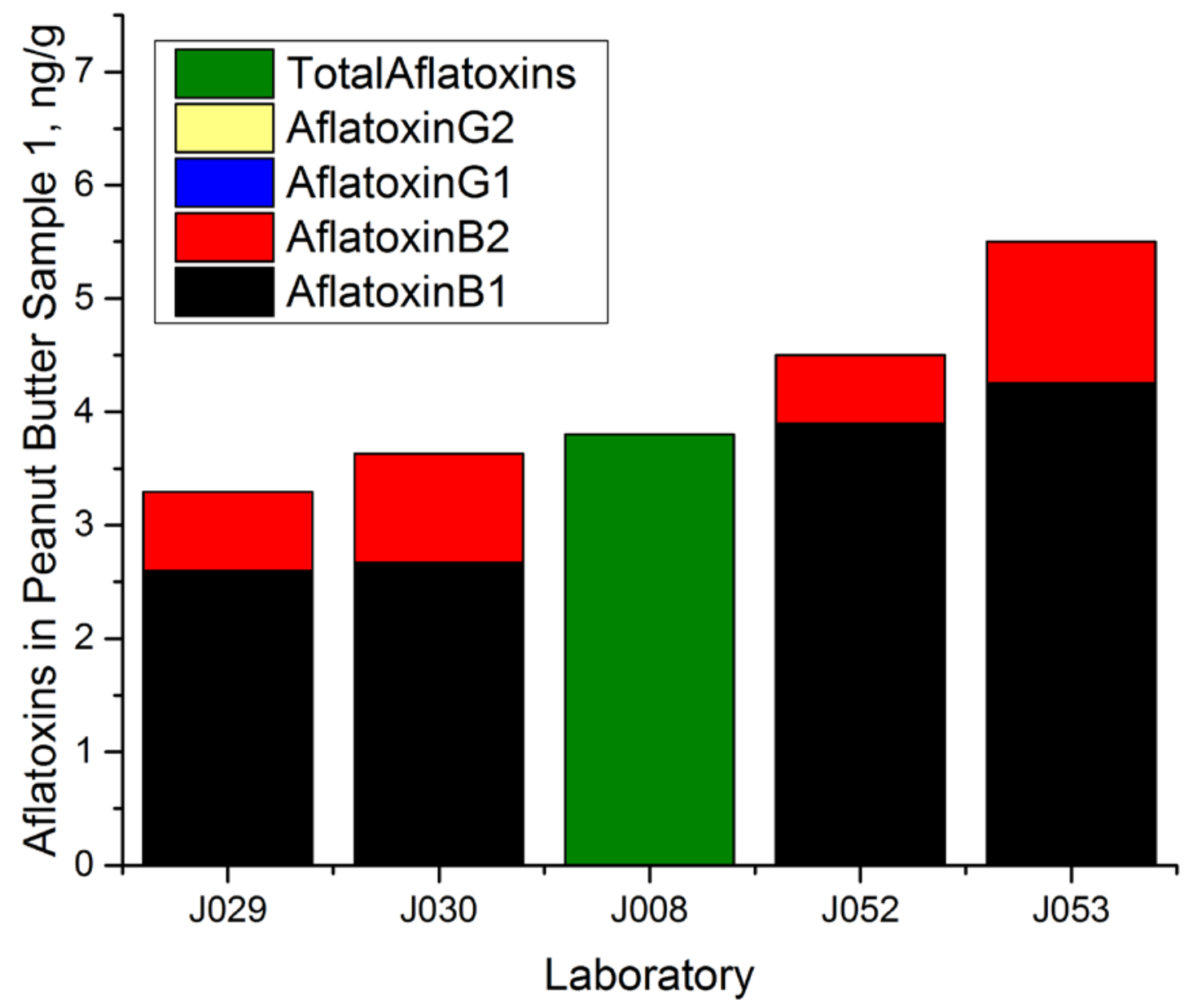

Figure 43. Total aflatoxins in peanut butter sample 1 (low) (composition view). In this view, total composition of the sample is plotted as a function of the measurement of individual components. The estimated value for total aflatoxins in this sample is $(5.23 \pm 2.22) \mathrm{ng} / \mathrm{g}$. 


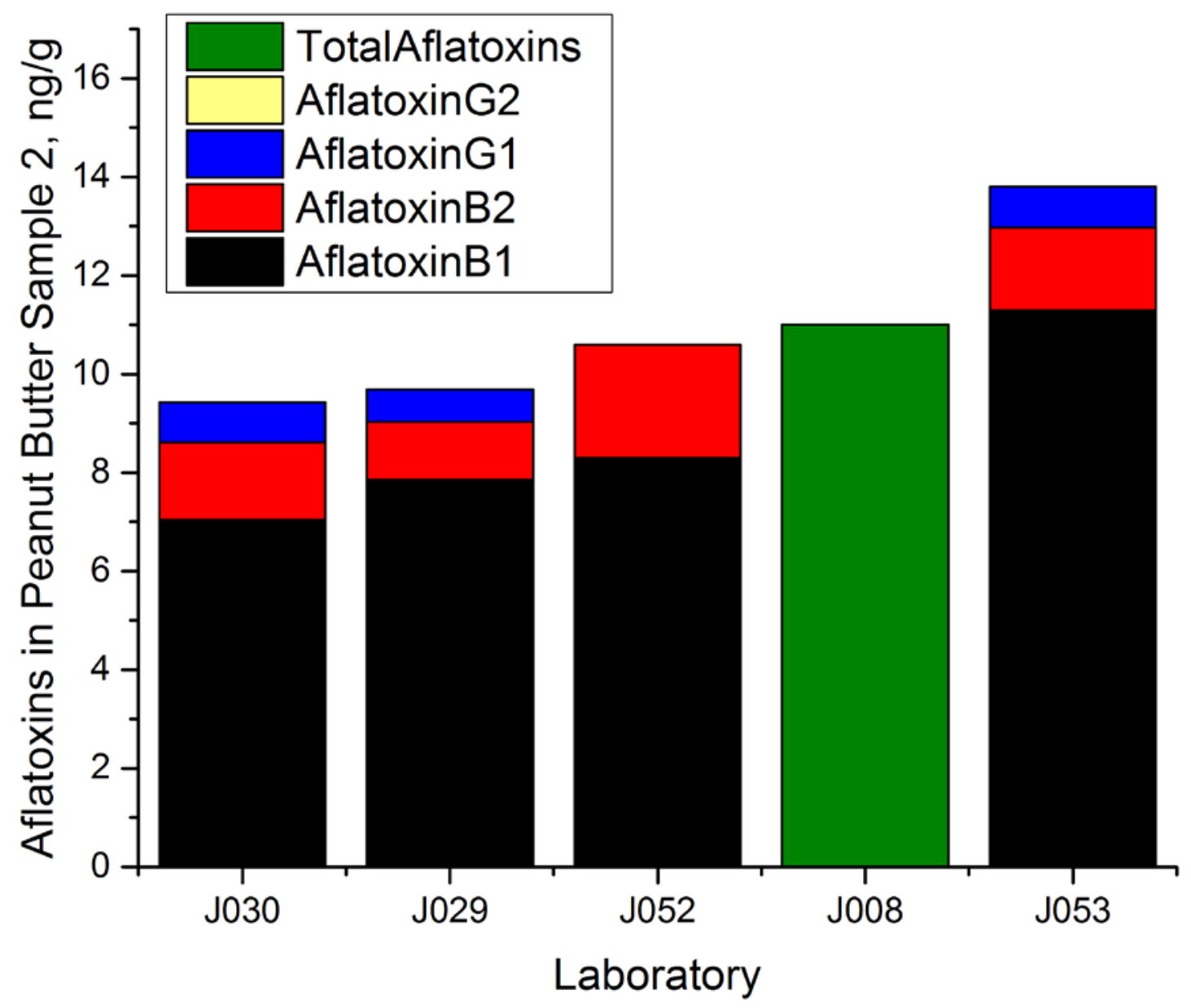

Figure 44. Total aflatoxins in peanut butter sample 2 (medium) (composition view). In this view, total composition of the sample is plotted as a function of the measurement of individual components. The estimated value for total aflatoxins in this sample is $(9.95 \pm 1.43) \mathrm{ng} / \mathrm{g}$. 


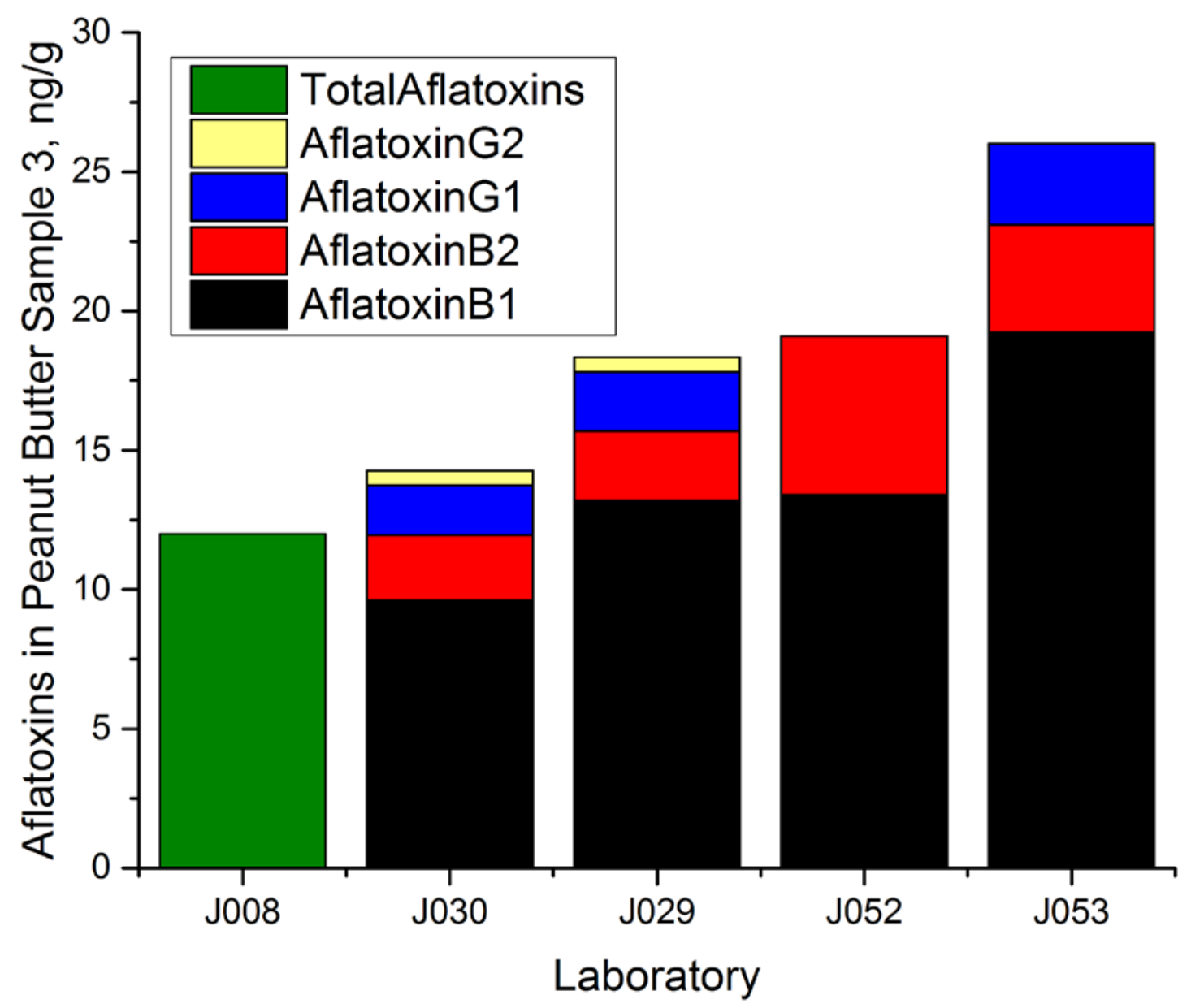

Figure 45. Total aflatoxins in peanut butter sample 3 (high) (composition view). In this view, total composition of the sample is plotted as a function of the measurement of individual components. The estimated value for total aflatoxins in this sample is $(14.8 \pm 2.9) \mathrm{ng} / \mathrm{g}$. 


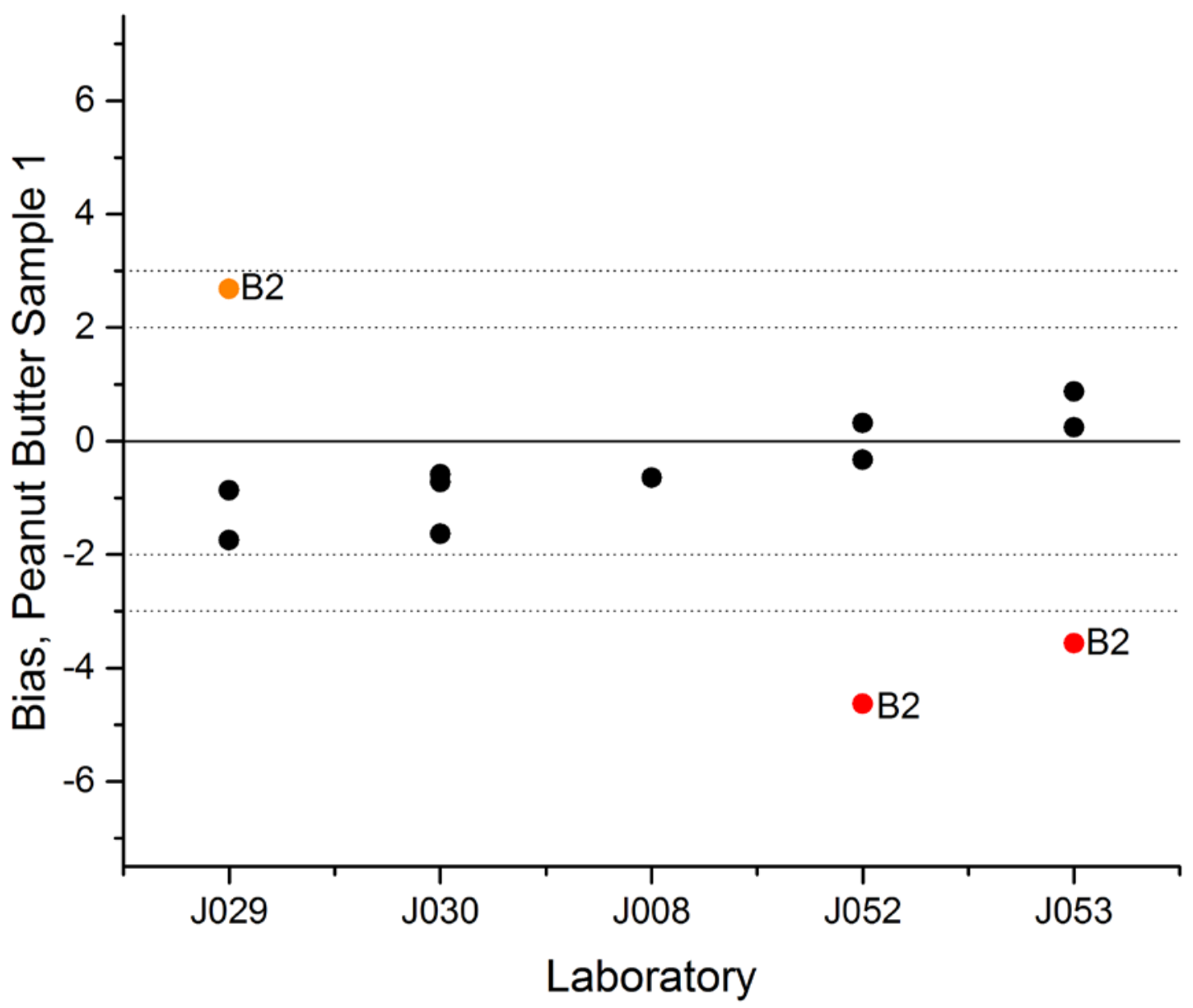

Figure 46. Aflatoxins in peanut butter sample 1 (low) (bias view). In this view, the $\mathrm{Z}_{\mathrm{NIST}}$-score for each individual component as well as total aflatoxins are plotted. Values determined to be marginally different than the NIST target value $(2<|\mathrm{Z}|<3)$ are colored in orange. Values determined to be significantly different than the NIST target value $(|\mathrm{Z}|>3)$ are colored in red. 


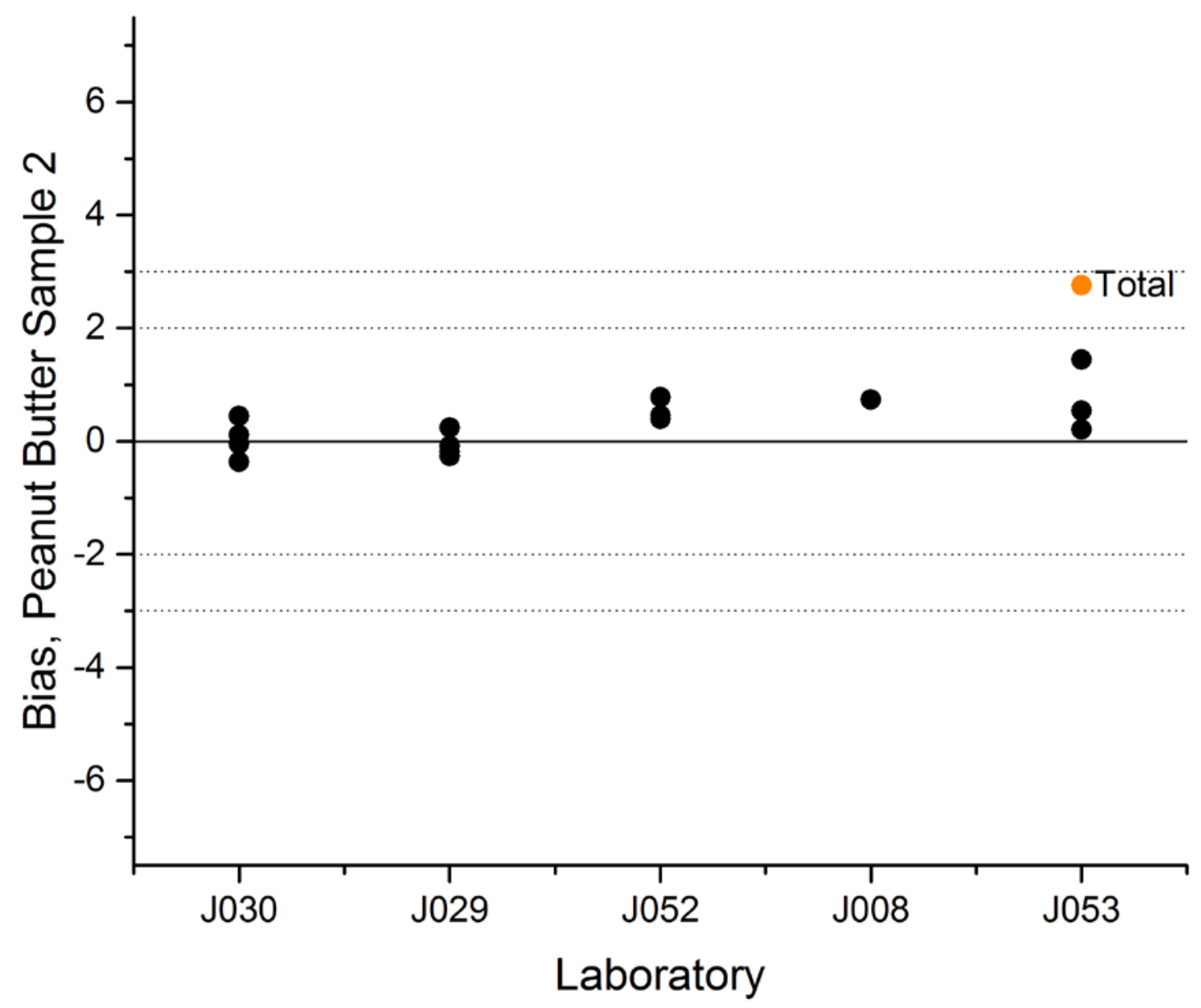

Figure 47. Aflatoxins in peanut butter sample 3 (medium) (bias view). In this view, the $\mathrm{Z}_{\mathrm{NIST}}$ score for each individual component as well as total aflatoxins are plotted. Values determined to be marginally different than the NIST target value $(2<|\mathrm{Z}|<3)$ are colored in orange. Values determined to be significantly different than the NIST target value $(|Z|>3)$ are colored in red. 


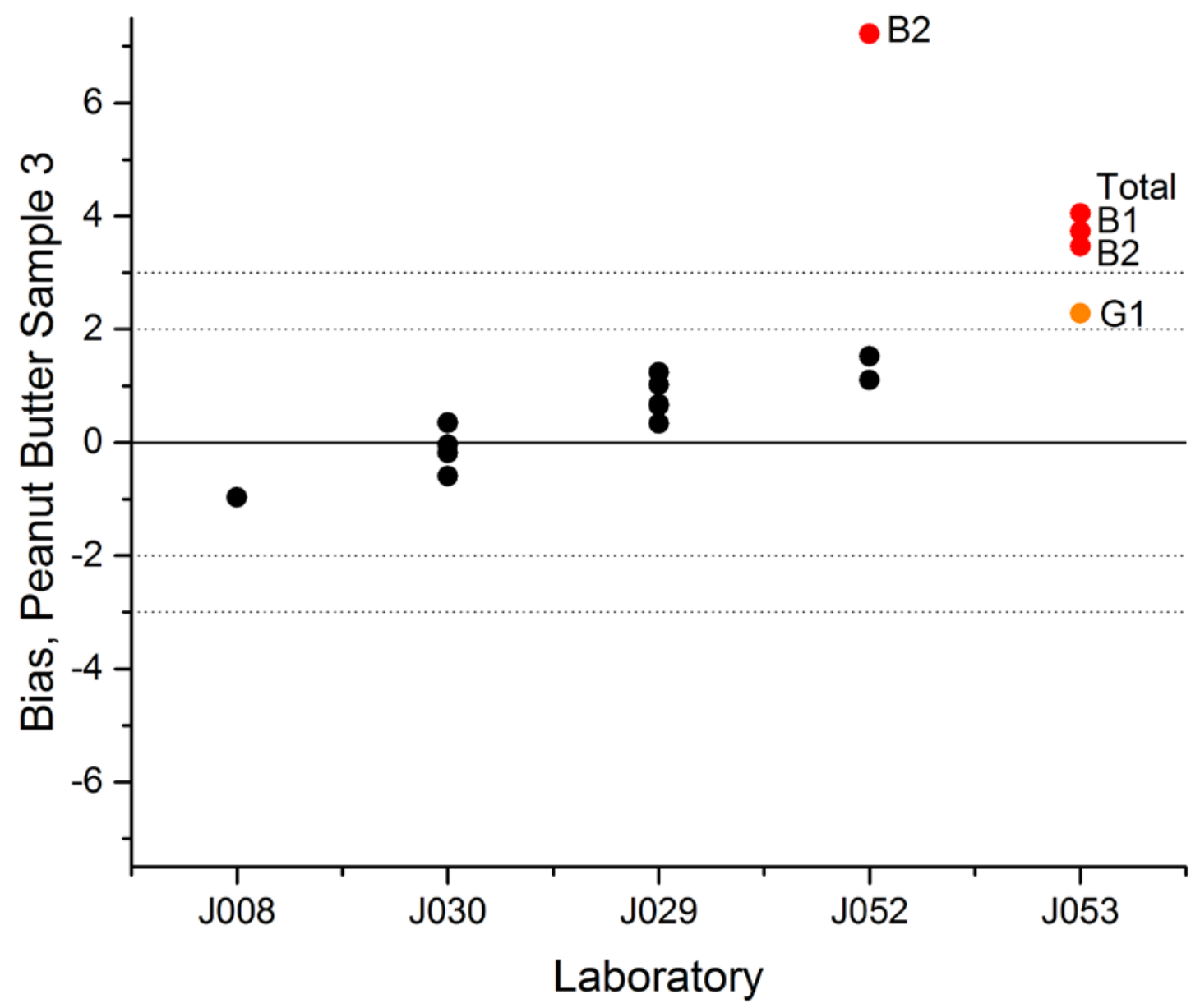

Figure 48. Aflatoxins in peanut butter sample 3 (high) (bias view). In this view, the $\mathrm{Z}_{\mathrm{NIST}}$-Score for each individual component as well as total aflatoxins are plotted. Values determined to be marginally different than the NIST target value $(2<|\mathrm{Z}|<3)$ are colored in orange. Values determined to be significantly different than the NIST target value $(|\mathrm{Z}|>3)$ are colored in red. 


\section{ISOFLAVONES IN SOY PRODUCTS}

\section{Study Overview}

In this study, participants were provided with two NIST SRMs, SRM 3236 Soy Protein Isolate and SRM 3234 Soy Flour. Participants were asked to use in-house analytical methods to determine the mass fractions of isoflavones (daidzin, glycitin, genistin, daidzein, glycitein, and genistein) in each of the matrices and report values on an as-received basis.

\section{Sample Information}

Soy Protein Isolate. Participants were provided with three packets, each containing $10 \mathrm{~g}$ of soy protein isolate. Before use, participants were instructed to mix each packet thoroughly and a sample size of at least $100 \mathrm{mg}$ was recommended. Participants were asked to prepare one sample and report as many analytes as possible from each packet provided, and to store the material at room temperature, $10{ }^{\circ} \mathrm{C}$ to $30^{\circ} \mathrm{C}$. Approximate analyte levels were not reported to participants prior to the study. The NIST certified values and uncertainties for isoflavones in SRM 3236 were determined using LC/abs and ID-LC/MS following solvent extraction and basic hydrolysis, and are reported in the table below both on a dry-mass basis and after correction for moisture of the material (4.83\%).

\begin{tabular}{|c|c|c|}
\hline Analyte & $\begin{array}{l}\text { Certified Mass Fraction } \\
\text { in SRM } 3236(\mu \mathrm{g} / \mathrm{g}) \\
\text { (dry-mass basis) }\end{array}$ & $\begin{array}{c}\text { Certified Mass Fraction } \\
\text { in SRM } 3236 \text { ( } \mu \mathrm{g} / \mathrm{g}) \\
\text { (as-received basis) }\end{array}$ \\
\hline Daidzin & $174 \pm 23$ & $165 \pm 22$ \\
\hline Glycitin & $31.37 \pm 0.52$ & $29.85 \pm 0.49$ \\
\hline Genistin & $329 \pm 10$ & \pm 10 \\
\hline Daidzein & $104.31 \pm 0.48$ & $99.27 \pm 0.46$ \\
\hline Glycitein & $22.71 \pm 0.19$ & $21.61 \pm$ \\
\hline Genistein & \pm 14 & \pm 13 \\
\hline Total Isoflavones & \pm 29 & \pm 27 \\
\hline
\end{tabular}

Soy Flour. Participants were provided one $50 \mathrm{~g}$ bottle of defatted soy flour. Before use, participants were instructed to mix the bottle thoroughly and a sample size of at least $100 \mathrm{mg}$ was recommended. Participants were asked to prepare three samples and report as many analytes as possible from the bottle provided, and to store the material at room temperature, $10{ }^{\circ} \mathrm{C}$ to $30^{\circ} \mathrm{C}$. Approximate analyte levels were not reported to participants prior to the study. The NIST estimated values for isoflavones in SRM 3234 were determined using LC/abs and IDLC/MS following solvent extraction and basic hydrolysis, and are reported in the table below on an as-received basis with an estimated uncertainty calculated as the standard deviation between the means of values determined by each method. 
NIST Estimated Mass Fraction

in SRM 3234 (mg/g)

Analyte

Daidzin

Glycitin

Genistin

Daidzein

Glycitein

Genistein

Total Isoflavones (as-received basis)

$\begin{array}{rrr}1693 & \pm & 427 \\ 241 & \pm & 31 \\ 2011 & \pm & 387 \\ 13.5 & \pm & 1.9 \\ & & \\ 14.9 & \pm & 0.1 \\ 3973 & \pm & 578\end{array}$

\section{Study Results}

- Sixteen laboratories enrolled in this exercise and received samples. Eleven laboratories reported data for at least some of the isoflavones in the study (69 \% participation).

- Laboratories reported using either solvent extraction (64\%) or solvent extraction with hydrolysis (36 \%) as the sample preparation method.

- A majority of the laboratories reported using LC/abs (91 \%) for isoflavone determination. One laboratory reported using DART-MS as their instrumental method.

- The consensus means were within the target ranges with acceptable between-laboratory variability for daidzein and genistein in the soy protein isolate (4 \% RSD for both analytes).

- The consensus means were above the target ranges for glycitein in the soy protein isolate and daidzein and genistein in the soy flour with high between-laboratory variability (29 $\%$ to $94 \%$ RSD).

- A clear distinction was observed for the glycoside (daidzin, glycitin, and genistin) results in both materials between the laboratories using solvent extraction compared to those including a hydrolysis step in their sample preparation.

o Separate consensus means and ranges were calculated for each sample preparation approach. The variances of each approach were found to be statistically equivalent, yet the mean values determined by each method for each analyte below were determined to be statistically different.

o With the exception of glycitin in the soy protein isolate, the consensus means for all of the glycosides as well as total isoflavones determined using hydrolysis were within the target ranges in both materials with excellent between-laboratory variability (5\% to $14 \%$ RSD).

o The consensus mean for glycitin in the soy protein isolate for laboratories using hydrolysis was above the target range with slightly higher between-laboratory variability (24\% RSD).

o For laboratories using an extraction approach (without hydrolysis), consensus means for all isoflavones were below the target ranges. Between-laboratory variability was higher than when hydrolysis was used ( $21 \%$ to $58 \%$ RSD), with the exception of total isoflavones in the soy protein isolate (7\% RSD). 
Analyte

Soy Protein Isolate

Daidzin

Glycitin

Genistin

Total Isoflavones

\section{Soy Flour}

Daidzin
Glycitin
Genistin
Total Isoflavones

\section{Total Isoflavones}

\author{
Consensus for Laboratories \\ Using Extraction ( $\mu \mathrm{g} / \mathrm{g})$
}

Consensus for Laboratories

Using Extraction with

Hydrolysis $(\mu \mathrm{g} / \mathrm{g})$

$\begin{array}{ccc}70 & \pm & 18 \\ 13.2 & \pm & 5.6 \\ 105 & \pm & 26 \\ 471 & \pm & 31\end{array}$

$\begin{aligned} 520 & \pm 100 \\ 91 & \pm 35 \\ 530 & \pm 200 \\ 1040 & \pm 600\end{aligned}$

$172 \pm 15$

$32.8 \pm 8.0$

$308 \pm 14$

$750 \pm 100$

$\begin{array}{rlr}1780 & \pm 170 \\ 276 & \pm 25 \\ 2200 & \pm 130 \\ 4220 & \pm 420\end{array}$

\section{Technical Recommendations}

The following recommendations are based on results obtained by the participants in this study.

- A clear distinction was observed for the glycoside (daidzin, glycitin, and genistin) results in both materials between the laboratories using solvent extraction compared to those including a hydrolysis step in their sample preparation.

o The hydrolysis step is utilized to convert the acetyl- and malonyl-glycosides of daidzin, glycitin, and genistin to their glycoside forms.

o If acetyl- and malonyl-glycosides are present, the use of hydrolysis in the sample preparation will result in determination of higher concentrations of the glycosides than if hydrolysis is not used.

o Because the hydrolysis step does not cleave the glycosides from the isoflavone molecules, no increase in concentration is observed for the aglycones (daidzein, glycitein, and genistein) when hydrolysis is utilized.

o Laboratories that do not use a hydrolysis step must take extra precautions to provide accurate results.

- Ensure that acetyl- and malonyl-glycosides are not being cleaved during the extraction process, or that the extraction process being utilized is robust enough to provide consistent results with expected deviations in laboratory practice (extraction time, temperature, solvent concentration, etc.).

- Ensure that the acetyl- and malonyl-glycosides are sufficiently stable so they do not degrade during the course of the sample preparation and chromatographic runs, leading to inconsistent results.

- Ensure that the separation method is adequate to resolve malonyl- and acetylglycosides from aglycones and glycosides so that coelutions do not cause falsely high results.

- The most accurate chromatographic peak identification and quantitation relies on pure reference standards. If standards are not available for every acetyl- and malonyl-glycoside, ensure that quantitation using response of another compound is appropriate.

o Because the NIST target values were determined using a hydrolysis step, laboratories that also utilized the hydrolysis step were in better agreement. However, laboratories 
using only extraction are not incorrect, and it is therefore important to specify method information when discussing and reporting isoflavone concentrations.

- A calibration issue may also be underlying the data for the glycosides (daidzin, glycitin, and genistin), as observed in the sample/control comparison views. The diagonal line observed is somewhat pronounced due to the effect of sample processing (described above), but for those laboratories using only solvent extraction, there may be some evidence of a calibration error.

o Be sure to confirm the purity of all reference standards by measuring moisture as well as checking chromatographic purity. NMR can provide additional information.

o The best approach is to use a reference standard for each isoflavone in the calibration.

o If an internal standard approach is used, confirm that there are no chromatographic coelutions with the internal standard by running a blank. 
Table 25. Individual data summary table (NIST) for isoflavones in soy dietary supplements.

\section{National Institute of Standards \& Technology}

\begin{tabular}{ccc} 
& & \\
& Lab Code: & NIST \\
\hline Analyte & Sample & Units \\
\hline Daidzin & Isolate & $\mu \mathrm{g} / \mathrm{g}$ \\
Daidzin & Flour & $\mu \mathrm{g} / \mathrm{g}$ \\
\hline Glycitin & Isolate & $\mu \mathrm{g} / \mathrm{g}$ \\
Glycitin & Flour & $\mu \mathrm{g} / \mathrm{g}$ \\
\hline Genistin & Isolate & $\mu \mathrm{g} / \mathrm{g}$ \\
Genistin & Flour & $\mu \mathrm{g} / \mathrm{g}$ \\
\hline Daidzein & Isolate & $\mu \mathrm{g} / \mathrm{g}$ \\
Daidzein & Flour & $\mu \mathrm{g} / \mathrm{g}$ \\
\hline Glycitein & Isolate & $\mu \mathrm{g} / \mathrm{g}$ \\
Glycitein & Flour & $\mu \mathrm{g} / \mathrm{g}$ \\
\hline Genistein & Isolate & $\mu \mathrm{g} / \mathrm{g}$ \\
Genistein & Flour & $\mu \mathrm{g} / \mathrm{g}$ \\
\hline Total Isoflavones & Isolate & $\mu \mathrm{g} / \mathrm{g}$ \\
Total Isoflavones & Flour & $\mu \mathrm{g} / \mathrm{g}$ \\
\hline
\end{tabular}

\section{Exercise J - May 2013 - Isoflavones}

\begin{tabular}{cccc}
\multicolumn{4}{c}{ 1. Your Results } \\
\hline $\mathrm{x}_{\mathrm{i}}$ & $\mathrm{S}_{\mathrm{i}}$ & $\mathrm{Z}_{\text {comm }}$ & $\mathrm{Z}_{\mathrm{NIST}}$ \\
\hline 165 & 22 & 0.9 & 0.0 \\
1693 & 427 & 0.9 & 0.0 \\
\hline 29.9 & 0.5 & 0.9 & 0.0 \\
241 & 31 & 0.8 & 0.0 \\
\hline 313 & 10 & 1.1 & 0.0 \\
2011 & 387 & 0.8 & 0.0 \\
\hline 99.3 & 0.5 & 0.0 & 0.0 \\
13.5 & 1.9 & -0.7 & 0.0 \\
\hline 21.6 & 0.2 & -0.4 & 0.0 \\
& & & \\
\hline 174 & 13 & 0.9 & 0.0 \\
14.9 & 0.1 & -0.4 & 0.0 \\
\hline 804 & 27 & 1.2 & 0.0 \\
3973 & 578 & 0.9 & 0.0 \\
\hline
\end{tabular}

\begin{tabular}{ccc}
\multicolumn{3}{c}{ 2. Community Results } \\
\hline $\mathrm{N}$ & $\mathrm{x}^{*}$ & $\mathrm{~s}^{*}$ \\
\hline 10 & 111 & 62 \\
10 & 1050 & 727 \\
\hline 9 & 19.2 & 11.4 \\
9 & 159 & 105 \\
\hline 10 & 189 & 118 \\
10 & 1220 & 968 \\
\hline 9 & 99.1 & 13.6 \\
10 & 37.8 & 35.7 \\
\hline 9 & 24.9 & 8.1 \\
4 & 149.0 & 285.0 \\
\hline 10 & 155 & 21 \\
10 & 16.6 & 4.8 \\
\hline 10 & 585 & 176 \\
11 & 2300 & 1800 \\
\hline
\end{tabular}

\begin{tabular}{cc}
\multicolumn{2}{c}{ 3. Target } \\
\hline $\mathrm{x}_{\mathrm{NIST}}$ & $U_{95}$ \\
\hline 165 & 22 \\
1693 & 427 \\
\hline 29.9 & 0.5 \\
241 & 31 \\
\hline 313 & 10 \\
2011 & 387 \\
\hline 99.3 & 0.5 \\
13.5 & 1.9 \\
\hline 21.6 & 0.2 \\
& \\
\hline 174 & 13 \\
14.9 & 0.1 \\
\hline 804 & 27 \\
3973 & 578 \\
\hline
\end{tabular}

$\mathrm{x}_{\mathrm{i}}$ Mean of reported values

$s_{i}$ Standard deviation of reported values

$\mathrm{N}$ Number of quantitative

$\mathrm{x}_{\text {NIST }}$ NIST-assessed value

$\mathrm{Z}_{\text {comm }}$ Z-score with respect to community consensus

values reported

$\mathrm{X}^{*}$ Robust mean of reported values

$U_{95} \pm 95 \%$ confidence interval about the assessed value or standard deviation $\left(\mathrm{s}_{\mathrm{NIST}}\right)$

$\mathrm{Z}_{\mathrm{NIST}}$ Z-score with respect to NIST value

s* Robust standard deviation 
Table 26. Data summary table for daidzin in soy dietary supplements.

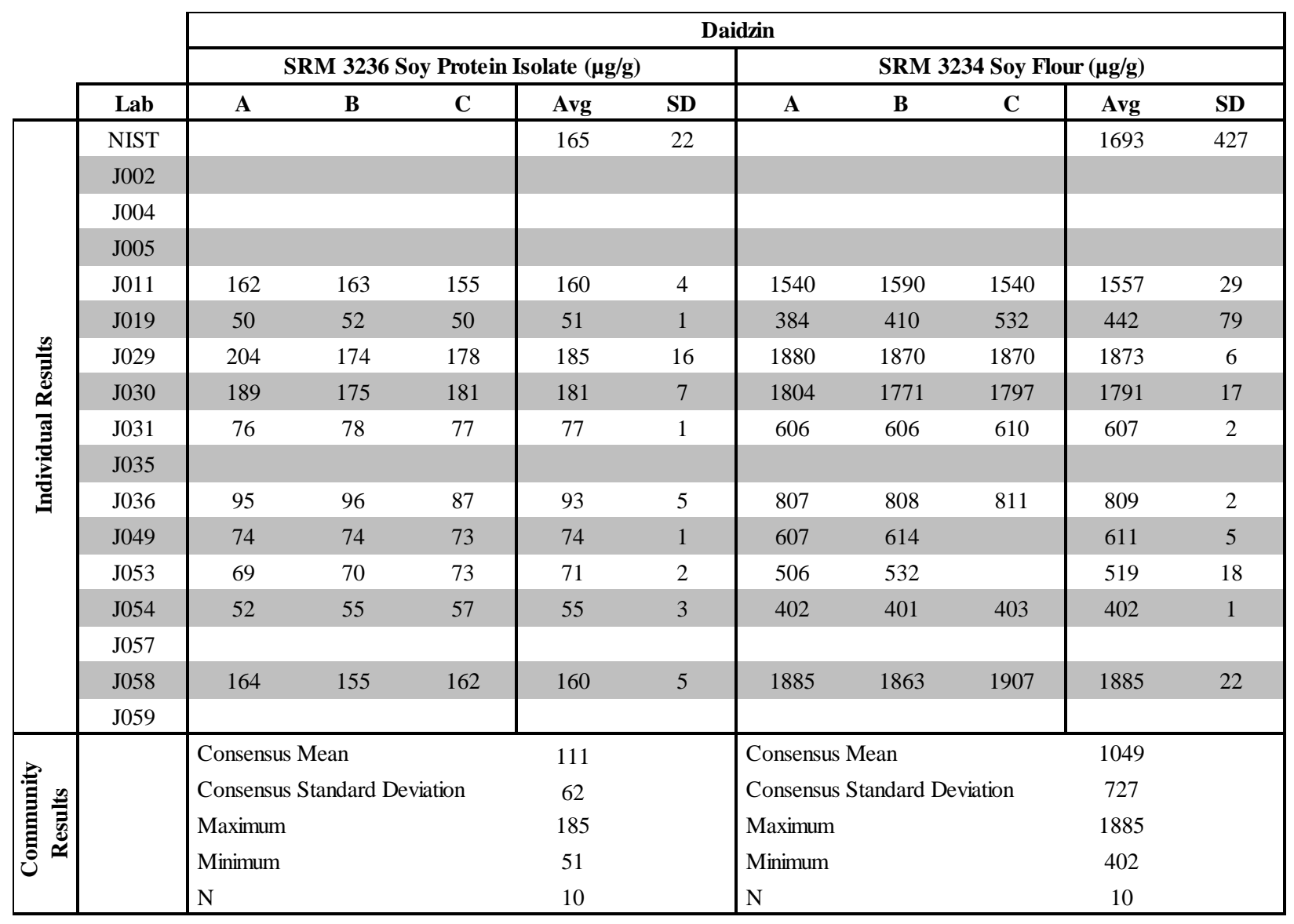


Table 27. Data summary table for glycitin in soy dietary supplements.

\begin{tabular}{|c|c|c|c|c|c|c|c|c|c|c|c|}
\hline & \multirow[b]{3}{*}{ Lab } & \multicolumn{10}{|c|}{ Glycitin } \\
\hline & & \multicolumn{10}{|c|}{ SRM 3236 Soy Protein Isolate ( $\mu \mathrm{g} / \mathrm{g}$ ) } \\
\hline & & A & B & C & Avg & SD & $\mathbf{A}$ & B & C & Avg & SD \\
\hline \multirow{17}{*}{ 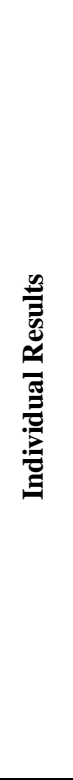 } & NIST & & & & 29.9 & 0.5 & & & & 241 & 31 \\
\hline & J002 & & & & & & & & & & \\
\hline & J004 & & & & & & & & & & \\
\hline & J005 & & & & & & & & & & \\
\hline & J011 & & & & & & & & & & \\
\hline & J019 & 11.2 & 10.6 & 10.5 & 10.8 & 0.4 & 91 & 92 & 101 & 94 & 6 \\
\hline & J029 & 31.4 & 28.5 & 28.8 & 29.6 & 1.6 & 258 & 259 & 257 & 258 & 1 \\
\hline & J030 & 39.3 & 38.8 & 44.6 & 40.9 & 3.2 & 308 & 295 & 299 & 301 & 7 \\
\hline & J031 & 16.0 & 17.1 & 18.3 & 17.1 & 1.2 & 106 & 106 & 107 & 106 & 1 \\
\hline & J035 & & & & & & & & & & \\
\hline & J036 & 17.1 & 17.5 & 15.1 & 16.6 & 1.3 & 153 & 154 & 150 & 152 & 2 \\
\hline & J049 & 13.0 & 12.1 & 11.1 & 12.1 & 1.0 & 81 & 85 & & 83 & 3 \\
\hline & J053 & 17.0 & 16.5 & 20.2 & 17.9 & 2.0 & 128 & 125 & & 126 & 3 \\
\hline & J054 & 5.0 & 5.0 & 5.0 & 5.0 & 0.0 & 44 & 43 & 45 & 44 & 1 \\
\hline & J057 & & & & & & & & & & \\
\hline & J058 & 29.3 & 27.2 & 27.3 & 27.9 & 1.2 & 267 & 271 & 267 & 268 & 2 \\
\hline & J059 & & & & & & & & & & \\
\hline \multirow{5}{*}{ 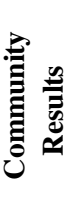 } & & \multicolumn{2}{|c|}{ Consensus Mean } & & \multicolumn{2}{|l|}{19.2} & \multicolumn{2}{|c|}{ Consensus Mean } & \multicolumn{2}{|r|}{159} & \\
\hline & & \multicolumn{3}{|c|}{ Consensus Standard Deviation } & \multicolumn{2}{|l|}{11.4} & \multicolumn{3}{|c|}{ Consensus Standard Deviation } & 105 & \\
\hline & & \multicolumn{3}{|c|}{ Maximum } & \multicolumn{2}{|l|}{40.9} & \multicolumn{3}{|c|}{ Maximum } & 301 & \\
\hline & & \multicolumn{3}{|c|}{ Minimum } & \multicolumn{2}{|l|}{5.0} & \multicolumn{3}{|c|}{ Minimum } & 44 & \\
\hline & & \multicolumn{3}{|l|}{$\mathrm{N}$} & \multicolumn{2}{|l|}{9} & \multicolumn{3}{|l|}{$\mathrm{N}$} & 9 & \\
\hline
\end{tabular}


Table 28. Data summary table for genistin in soy dietary supplements.

\begin{tabular}{|c|c|c|c|c|c|c|c|c|c|c|c|}
\hline & \multirow[b]{3}{*}{ Lab } & \multicolumn{10}{|c|}{ Genisitn } \\
\hline & & \multicolumn{5}{|c|}{ SRM 3236 Soy Protein Isolate $(\mu g / g)$} & \multicolumn{5}{|c|}{ SRM 3234 Soy Flour $(\mu \mathrm{g} / \mathrm{g})$} \\
\hline & & A & $\mathbf{B}$ & $\mathbf{C}$ & Avg & SD & $\mathbf{A}$ & B & $\mathbf{C}$ & Avg & SD \\
\hline \multirow{17}{*}{ 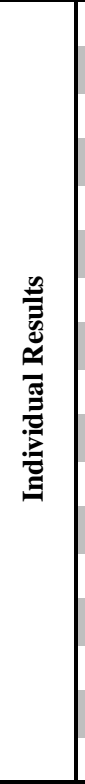 } & NIST & & & & 313 & 10 & & & & 2011 & 387 \\
\hline & J002 & & & & & & & & & & \\
\hline & J004 & & & & & & & & & & \\
\hline & J005 & & & & & & & & & & \\
\hline & J011 & 311 & 314 & 294 & 306 & 11 & 2110 & 2180 & 2100 & 2130 & 44 \\
\hline & J019 & 77 & 80 & 71 & 76 & 5 & 317 & 358 & 102 & 259 & 138 \\
\hline & J029 & 311 & 283 & 291 & 295 & 14 & 2080 & 2080 & 2080 & 2080 & 0 \\
\hline & J030 & 327 & 324 & 323 & 325 & 2 & 2260 & 2225 & 2258 & 2248 & 20 \\
\hline & $\mathrm{J} 031$ & 130 & 126 & 130 & 129 & 2 & 651 & 657 & 661 & 656 & 5 \\
\hline & J035 & & & & & & & & & & \\
\hline & J036 & 126 & 127 & 116 & 123 & 6 & 677 & 681 & 675 & 678 & 3 \\
\hline & J049 & 134 & 136 & 136 & 135 & 1 & 750 & 766 & & 758 & 11 \\
\hline & J053 & 114 & 111 & 111 & 112 & 1 & 592 & 620 & & 606 & 20 \\
\hline & J054 & 84 & 88 & 91 & 88 & 4 & 464 & 463 & 465 & 464 & 1 \\
\hline & J057 & & & & & & & & & & \\
\hline & J058 & 308 & 302 & 308 & 306 & 4 & 2344 & 2302 & 2352 & 2333 & 27 \\
\hline & J059 & & & & & & & & & & \\
\hline \multirow{5}{*}{ 象 } & & \multicolumn{2}{|c|}{ Consensus Mean } & & \multicolumn{2}{|l|}{189} & \multicolumn{2}{|c|}{ Consensus Mean } & \multicolumn{2}{|r|}{1221} & \\
\hline & & \multicolumn{3}{|c|}{ Consensus Standard Deviation } & \multicolumn{2}{|l|}{118} & \multicolumn{3}{|c|}{ Consensus Standard Deviation } & 968 & \\
\hline & & \multicolumn{3}{|c|}{ Maximum } & \multicolumn{2}{|l|}{325} & \multicolumn{3}{|c|}{ Maximum } & 2333 & \\
\hline & & \multicolumn{3}{|c|}{ Minimum } & \multicolumn{2}{|l|}{76} & \multicolumn{3}{|c|}{ Minimum } & 259 & \\
\hline & & & \multicolumn{2}{|l|}{10} & & 10 & \\
\hline
\end{tabular}


Table 29. Data summary table for daidzein in soy dietary supplements.

\begin{tabular}{|c|c|c|c|c|c|c|c|c|c|c|c|}
\hline & \multirow[b]{3}{*}{ Lab } & \multicolumn{10}{|c|}{ Daidzein } \\
\hline & & \multicolumn{5}{|c|}{ SRM 3236 Soy Protein Isolate $(\mu \mathrm{g} / \mathrm{g})$} & \multicolumn{5}{|c|}{ SRM 3234 Soy Flour ( $\mu \mathrm{g} / \mathrm{g})$} \\
\hline & & A & B & $\mathbf{C}$ & Avg & SD & A & B & $\mathbf{C}$ & Avg & SD \\
\hline \multirow{17}{*}{ 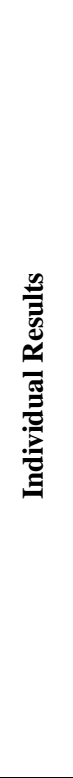 } & NIST & & & & 99.3 & 0.5 & & & & 13.5 & 1.9 \\
\hline & J002 & & & & & & & & & & \\
\hline & J004 & & & & & & & & & & \\
\hline & J005 & & & & & & & & & & \\
\hline & J011 & & & & & & & & & & \\
\hline & J019 & 119.6 & 120.6 & 117.6 & 119.2 & 1.6 & 63.4 & 66.7 & 81.9 & 70.7 & 9.9 \\
\hline & J029 & 79.7 & 83.8 & 87.3 & 83.6 & 3.8 & 15.6 & 15.0 & 15.4 & 15.3 & 0.3 \\
\hline & J030 & 116.0 & 117.1 & 120.3 & 117.8 & 2.2 & 35.2 & 29.1 & 28.9 & 31.1 & 3.6 \\
\hline & J031 & 91.9 & 92.0 & 92.1 & 92.0 & 0.1 & 16.5 & 13.2 & 13.9 & 14.5 & 1.7 \\
\hline & J035 & & & & & & & & & & \\
\hline & J036 & 98.3 & 98.3 & 98.6 & 98.4 & 0.2 & 17.0 & 16.9 & 16.6 & 16.8 & 0.2 \\
\hline & J049 & 93.7 & 93.0 & 92.5 & 93.1 & 0.6 & 85.0 & 83.0 & & 84.0 & 1.4 \\
\hline & J053 & 100.2 & 104.6 & 98.1 & 101.0 & 3.3 & 27.6 & 26.5 & & 27.1 & 0.8 \\
\hline & J054 & 94.0 & 92.0 & 92.0 & 92.7 & 1.2 & 11.1 & 11.3 & 11.0 & 11.1 & 0.2 \\
\hline & J057 & & & & & & & & & & \\
\hline & J058 & 94.9 & 91.9 & 95.0 & 93.9 & 1.8 & 16.8 & 15.9 & 15.7 & 16.1 & 0.6 \\
\hline & J059 & & & & & & 103.0 & 109.0 & 102.0 & 104.7 & 3.8 \\
\hline \multirow{5}{*}{ 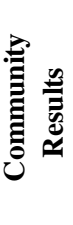 } & & \multicolumn{3}{|c|}{ Consensus Mean } & \multicolumn{2}{|l|}{99.1} & \multicolumn{2}{|c|}{ Consensus Mean } & & 37.8 & \\
\hline & & \multicolumn{3}{|c|}{ Consensus Standard Deviation } & \multicolumn{2}{|l|}{13.6} & \multicolumn{3}{|c|}{ Consensus Standard Deviation } & 35.7 & \\
\hline & & \multicolumn{3}{|c|}{ Maximum } & \multicolumn{2}{|l|}{119.2} & \multicolumn{3}{|c|}{ Maximum } & 104.7 & \\
\hline & & \multicolumn{3}{|c|}{ Minimum } & \multicolumn{2}{|l|}{83.6} & \multicolumn{3}{|l|}{ Minimum } & 11.1 & \\
\hline & & \multicolumn{3}{|l|}{$\mathrm{N}$} & \multicolumn{2}{|l|}{9} & \multicolumn{3}{|l|}{$\mathrm{N}$} & 10 & \\
\hline
\end{tabular}


Table 30. Data summary table for glycitein in soy dietary supplements.

\begin{tabular}{|c|c|c|c|c|c|c|c|c|c|c|c|}
\hline & \multirow[b]{3}{*}{ Lab } & \multicolumn{10}{|c|}{ Glycitein } \\
\hline & & \multicolumn{5}{|c|}{ SRM 3236 Soy Protein Isolate $(\mu \mathrm{g} / \mathrm{g})$} & \multicolumn{5}{|c|}{ SRM 3234 Soy Flour $(\mu \mathrm{g} / \mathrm{g})$} \\
\hline & & A & B & $\mathbf{C}$ & Avg & SD & A & B & $\mathbf{C}$ & Avg & SD \\
\hline \multirow{17}{*}{ 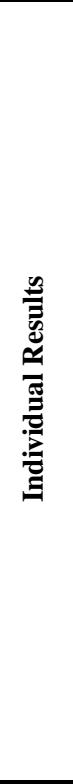 } & NIST & & & & 21.6 & 0.2 & & & & & \\
\hline & J002 & & & & & & & & & & \\
\hline & $\mathrm{J} 004$ & & & & & & & & & & \\
\hline & J005 & & & & & & & & & & \\
\hline & J011 & & & & & & & & & & \\
\hline & J019 & 18.7 & 19.0 & 18.7 & 18.8 & 0.2 & 1.5 & 1.7 & 2.7 & 2.0 & 0.6 \\
\hline & J029 & 18.7 & 18.1 & 18.4 & 18.4 & 0.3 & & & & & \\
\hline & J030 & 28.4 & 20.4 & 25.8 & 24.9 & 4.1 & 10.8 & 10.2 & 10.6 & 10.5 & 0.3 \\
\hline & J031 & 24.9 & 23.1 & 22.1 & 23.4 & 1.4 & & & & & \\
\hline & J035 & & & & & & & & & & \\
\hline & J036 & 47.2 & 46.6 & 46.3 & 46.7 & 0.5 & & & & & \\
\hline & J049 & 21.6 & 22.2 & 21.5 & 21.8 & 0.4 & & & & & \\
\hline & J053 & 23.9 & 25.7 & 25.8 & 25.1 & 1.1 & & & & & \\
\hline & J054 & 35.7 & 36.6 & 36.3 & 36.2 & 0.5 & 524.6 & 524.1 & 524.9 & 524.5 & 0.4 \\
\hline & J057 & & & & & & & & & & \\
\hline & J058 & 17.8 & 19.3 & 19.0 & 18.7 & 0.8 & & & & & \\
\hline & J059 & & & & & & 57.0 & 62.0 & 56.0 & 58.3 & 3.2 \\
\hline & & Consens & & & 24.9 & & Consenst & & & 148.8 & \\
\hline 鞄 & & Consens & ndard & tion & 8.1 & & Consenst & indard I & tion & 285.4 & \\
\hline 言 & & Maximu & & & 46.7 & & Maximun & & & 524.5 & \\
\hline$\tilde{u}$ & & Minimun & & & 18.4 & & Minimum & & & 2.0 & \\
\hline & & $\mathrm{N}$ & & & 9 & & $\mathrm{~N}$ & & & 4 & \\
\hline
\end{tabular}


Table 31. Data summary table for genistein in soy dietary supplements.

\begin{tabular}{|c|c|c|c|c|c|c|c|c|c|c|c|}
\hline & \multirow[b]{3}{*}{ Lab } & \multicolumn{10}{|c|}{ Genistein } \\
\hline & & \multicolumn{5}{|c|}{ SRM 3236 Soy Protein Isolate $(\mu \mathrm{g} / \mathrm{g})$} & \multicolumn{5}{|c|}{ SRM 3234 Soy Flour $(\mu \mathrm{g} / \mathrm{g})$} \\
\hline & & A & B & C & Avg & SD & A & B & C & Avg & SD \\
\hline \multirow{17}{*}{ 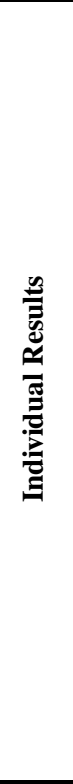 } & NIST & & & & 174 & 13 & & & & 14.9 & 0.1 \\
\hline & J002 & & & & & & & & & & \\
\hline & J004 & & & & & & & & & & \\
\hline & J005 & & & & & & & & & & \\
\hline & J011 & 169 & 170 & 159 & 166 & 6 & & & & & \\
\hline & J019 & 163 & 164 & 160 & 162 & 2 & 11.4 & 10.6 & 12.4 & 11.5 & 0.9 \\
\hline & J029 & 127 & 121 & 122 & 123 & 3 & 13.6 & 14.0 & 14.7 & 14.1 & 0.6 \\
\hline & J030 & 157 & 152 & 158 & 156 & 3 & 25.1 & 22.8 & 23.4 & 23.8 & 1.2 \\
\hline & J031 & 155 & 153 & 156 & 155 & 2 & 14.5 & 14.7 & 13.9 & 14.4 & 0.4 \\
\hline & J035 & & & & & & & & & & \\
\hline & J036 & 164 & 164 & 167 & 165 & 2 & 14.1 & 14.7 & 15.1 & 14.6 & 0.5 \\
\hline & J049 & 162 & 162 & 162 & 162 & 0 & 16.0 & 15.8 & & 15.9 & 0.1 \\
\hline & J053 & 121 & 120 & 123 & 121 & 1 & 17.9 & 18.2 & & 18.1 & 0.2 \\
\hline & J054 & 150 & 153 & 157 & 153 & 3 & 12.7 & 12.5 & 12.9 & 12.7 & 0.2 \\
\hline & J057 & & & & & & & & & & \\
\hline & J058 & 185 & 185 & 180 & 183 & 3 & 34.3 & 35.5 & 30.5 & 33.4 & 2.6 \\
\hline & J059 & & & & & & 19.0 & 15.0 & 18.0 & 17.3 & 2.1 \\
\hline \multirow{5}{*}{ 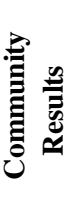 } & & \multicolumn{2}{|c|}{ Consensus Mean } & & \multicolumn{2}{|l|}{155} & \multicolumn{2}{|c|}{ Consensus Mean } & & 16.6 & \\
\hline & & \multicolumn{3}{|c|}{ Consensus Standard Deviation } & \multicolumn{2}{|l|}{21} & \multicolumn{3}{|c|}{ Consensus Standard Deviation } & 4.8 & \\
\hline & & \multicolumn{3}{|c|}{ Maximum } & \multicolumn{2}{|l|}{183} & \multicolumn{3}{|c|}{ Maximum } & 33.4 & \\
\hline & & \multicolumn{3}{|c|}{ Minimum } & \multicolumn{2}{|l|}{121} & \multicolumn{3}{|c|}{ Minimum } & 11.5 & \\
\hline & & \multicolumn{3}{|l|}{$\mathrm{N}$} & \multicolumn{2}{|l|}{10} & \multicolumn{3}{|l|}{$\mathrm{N}$} & 10 & \\
\hline
\end{tabular}


Table 32. Data summary table for total isoflavones in soy dietary supplements.

\begin{tabular}{|c|c|c|c|c|c|c|c|c|c|c|c|}
\hline & \multirow[b]{3}{*}{ Lab } & \multicolumn{10}{|c|}{ Total Isoflavones } \\
\hline & & \multicolumn{5}{|c|}{ SRM 3236 Soy Protein Isolate $(\mu g / g)$} & \multicolumn{5}{|c|}{ SRM 3234 Soy Flour $(\mu \mathrm{g} / \mathrm{g})$} \\
\hline & & A & $\mathbf{B}$ & $\mathbf{C}$ & Avg & SD & $\mathbf{A}$ & B & $\mathbf{C}$ & Avg & SD \\
\hline \multirow{17}{*}{ 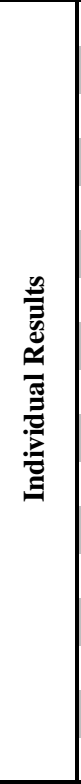 } & NIST & & & & 804 & 27 & & & & 3973 & 578 \\
\hline & J002 & & & & & & & & & & \\
\hline & J004 & & & & & & & & & & \\
\hline & J005 & & & & & & & & & & \\
\hline & J011 & 642 & 647 & 608 & 632 & 21 & 3650 & 3770 & 3640 & 3687 & 72 \\
\hline & J019 & 440 & 446 & 427 & 438 & 10 & 869 & 939 & 832 & 880 & 54 \\
\hline & J029 & 772 & 708 & 726 & 735 & 33 & 4247 & 4238 & 4237 & 4241 & 6 \\
\hline & J030 & 857 & 828 & 852 & 845 & 15 & 4443 & 4353 & 4416 & 4404 & 46 \\
\hline & J031 & 494 & 489 & 495 & 493 & 3 & 1394 & 1397 & 1406 & 1399 & 6 \\
\hline & J035 & & & & & & & & & & \\
\hline & J036 & 548 & 550 & 530 & 543 & 11 & 1668 & 1675 & 1668 & 1670 & 4 \\
\hline & J049 & 498 & 499 & 496 & 498 & 2 & 1539 & 1564 & & 1551 & 18 \\
\hline & J053 & 445 & 448 & 451 & 448 & 3 & 1271 & 1321 & & 1296 & 35 \\
\hline & J054 & 421 & 430 & 438 & 430 & 9 & 1459 & 1455 & 1462 & 1458 & 3 \\
\hline & J057 & & & & & & & & & & \\
\hline & J058 & 798 & 780 & 792 & 790 & 9 & 4548 & 4487 & 4572 & 4535 & 43 \\
\hline & J059 & & & & & & 179 & 186 & 176 & 180 & 5 \\
\hline \multirow{5}{*}{ 象 } & & \multicolumn{2}{|c|}{ Consensus Mean } & & 585 & & \multicolumn{2}{|c|}{ Consensus Mean } & & 2300 & \\
\hline & & \multicolumn{3}{|c|}{ Consensus Standard Deviation } & 176 & & \multicolumn{3}{|c|}{ Consensus Standard Deviation } & 1797 & \\
\hline & & \multicolumn{3}{|c|}{ Maximum } & 845 & & \multicolumn{3}{|c|}{ Maximum } & 4535 & \\
\hline & & \multicolumn{3}{|c|}{ Minimum } & 430 & & \multicolumn{3}{|c|}{ Minimum } & 180 & \\
\hline & & & 10 & & & 11 & \\
\hline
\end{tabular}




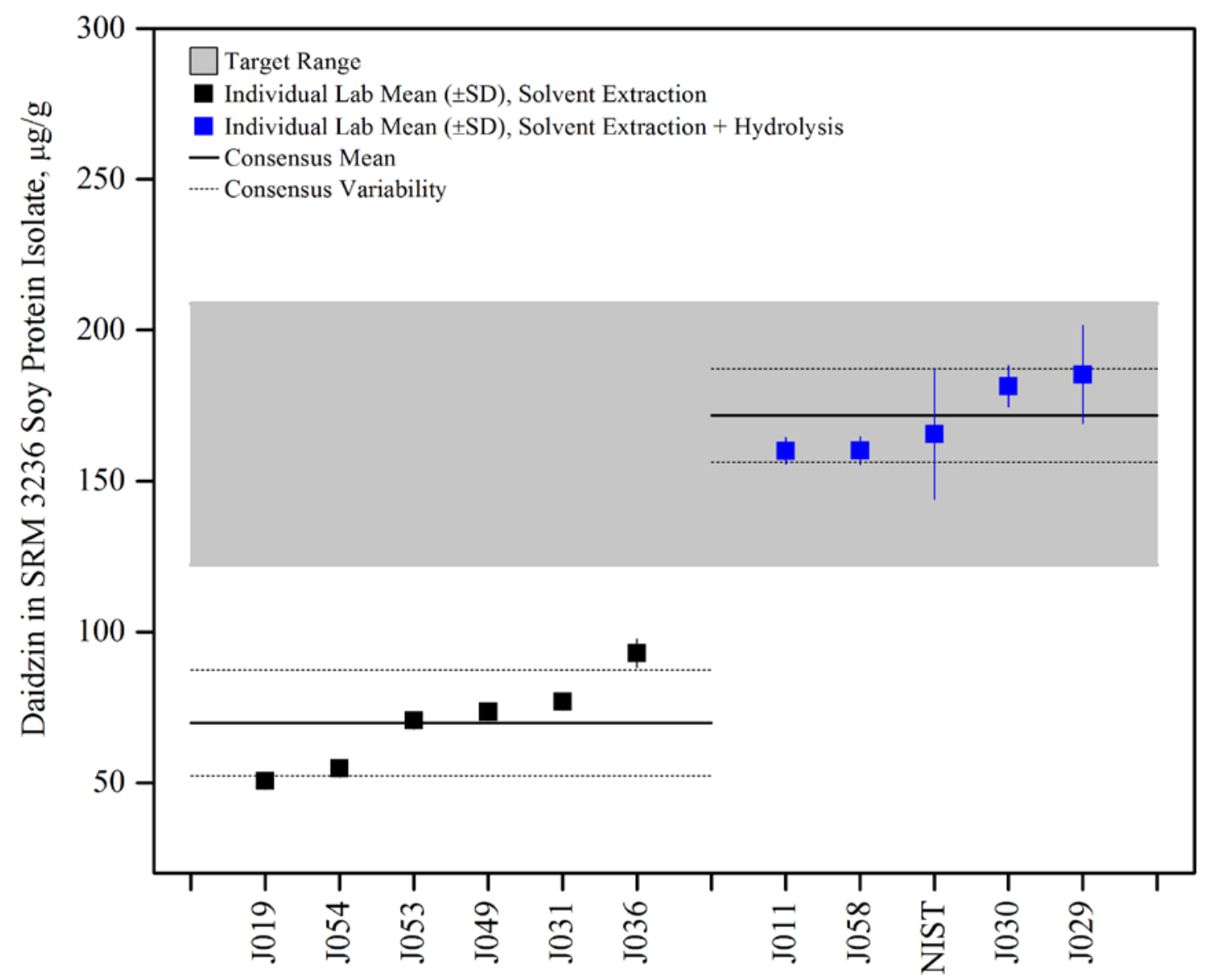

Figure 49. Daidzin in SRM 3236 Soy Protein Isolate (data summary view, sample preparation comparison). In this view, individual laboratory data are plotted with the individual laboratory standard deviation (error bars). The color of the data point represents the sample preparation method employed. The black solid lines represent the consensus means for each sample preparation method, and the black dotted lines represent the consensus variability calculated as one standard deviation about the consensus mean for that sample preparation method. The gray shaded region represents the target zone for "acceptable" performance, which encompasses the NIST certified value bounded by twice its uncertainty $\left(U_{95}\right)$. 


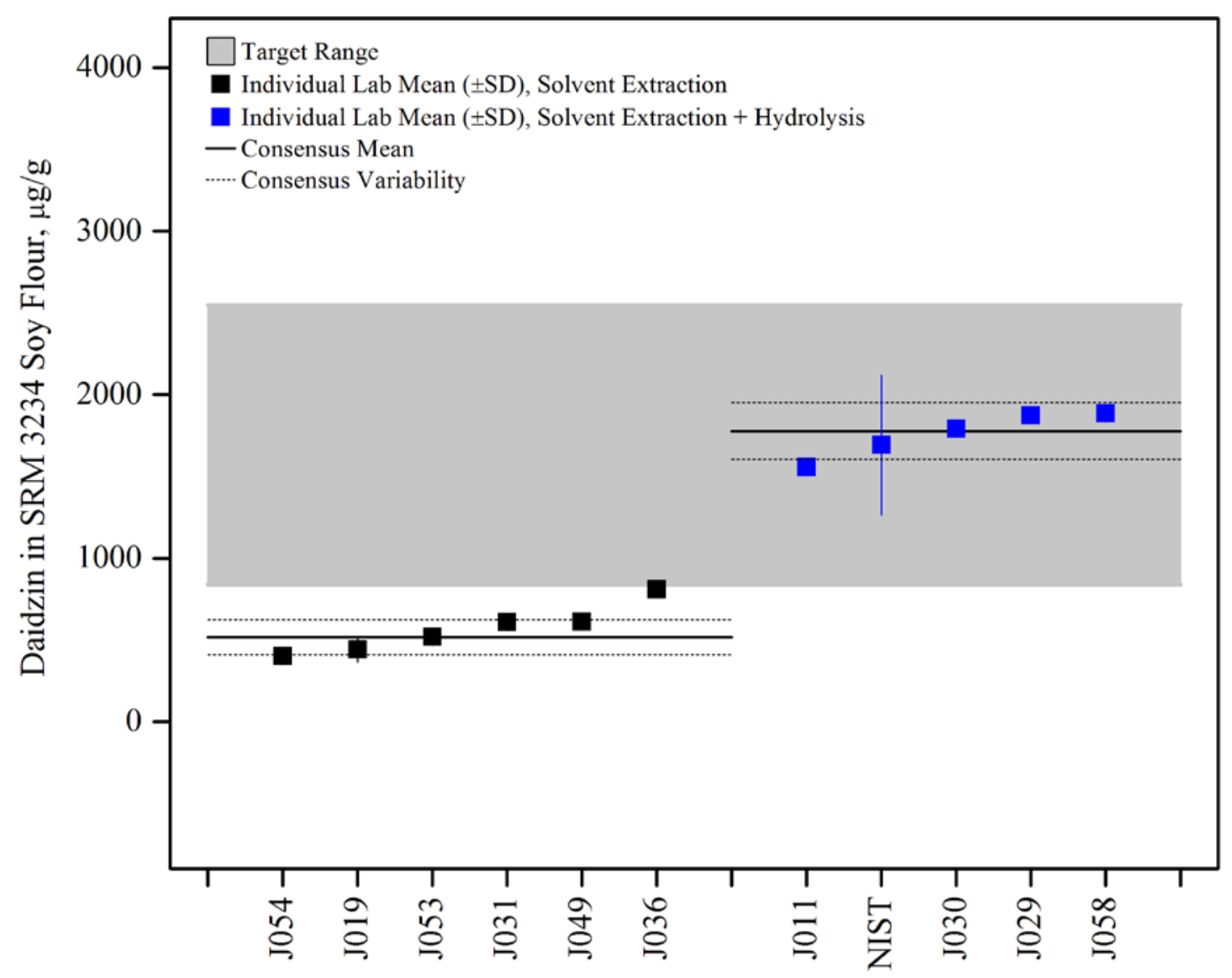

Figure 50. Daidzin in SRM 3234 Soy Flour (data summary view, sample preparation comparison). In this view, individual laboratory data are plotted with the individual laboratory standard deviation (error bars). The color of the data point represents the sample preparation method employed. The black solid lines represents the consensus mean for each sample preparation method, and the black dotted lines represent the consensus variability calculated as one standard deviation about the consensus mean for that sample preparation method. The gray shaded region represents the target zone for "acceptable" performance, which encompasses the NIST value determined by LC/abs and ID-LC/MS, bounded by an estimated uncertainty calculated as the standard deviation between the means of values determined by each method. 


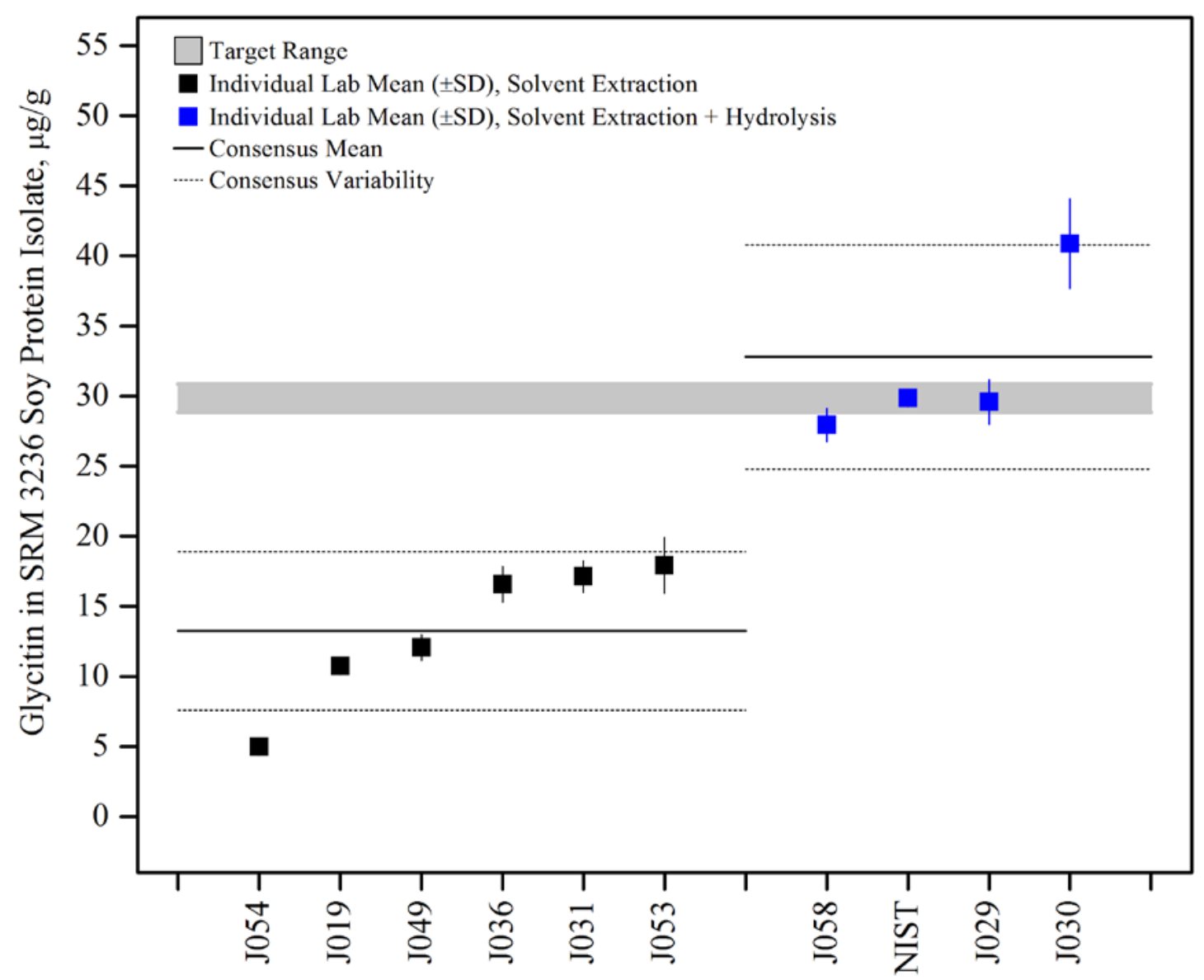

Figure 51. Glycitin in SRM 3236 Soy Protein Isolate (data summary view, sample preparation comparison). In this view, individual laboratory data are plotted with the individual laboratory standard deviation (error bars). The color of the data point represents the sample preparation method employed. The black solid lines represents the consensus mean for each sample preparation method, and the black dotted lines represent the consensus variability calculated as one standard deviation about the consensus mean for that sample preparation method. The gray shaded region represents the target zone for "acceptable” performance, which encompasses the NIST certified value bounded by twice its uncertainty $\left(U_{95}\right)$. 


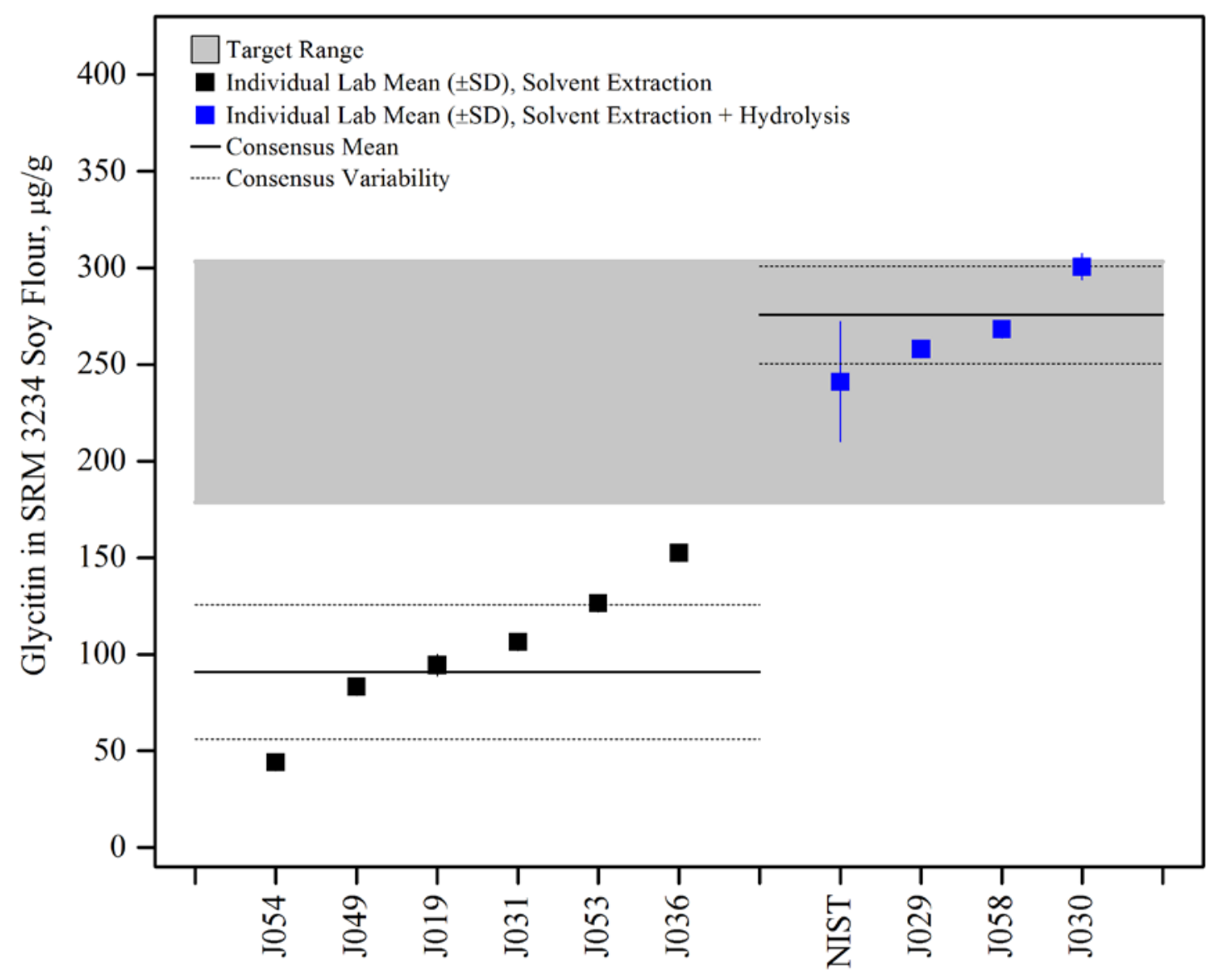

Figure 52. Glycitin in SRM 3234 Soy Flour (data summary view, sample preparation comparison). In this view, individual laboratory data are plotted with the individual laboratory standard deviation (error bars). The color of the data point represents the sample preparation method employed. The black solid lines represents the consensus mean for each sample preparation method, and the black dotted lines represent the consensus variability calculated as one standard deviation about the consensus mean for that sample preparation method. The gray shaded region represents the target zone for "acceptable" performance, which encompasses the NIST value determined by LC/abs and ID-LC/MS, bounded by an estimated uncertainty calculated as the standard deviation between the means of values determined by each method. 


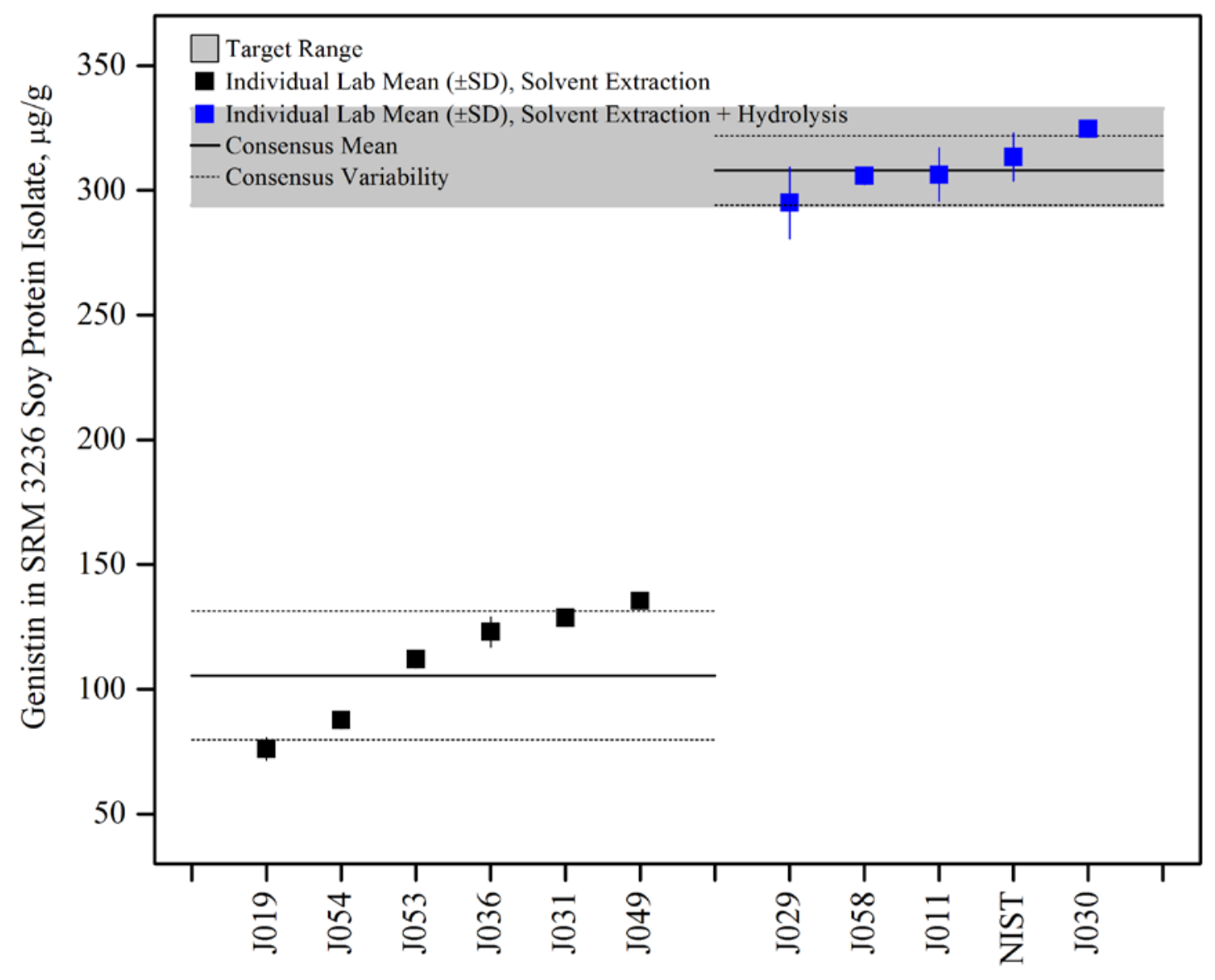

Figure 53. Genistin in SRM 3236 Soy Protein Isolate (data summary view, sample preparation comparison). In this view, individual laboratory data are plotted with the individual laboratory standard deviation (error bars). The color of the data point represents the sample preparation method employed. The black solid lines represents the consensus mean for each sample preparation method, and the black dotted lines represent the consensus variability calculated as one standard deviation about the consensus mean for that sample preparation method. The gray shaded region represents the target zone for "acceptable" performance, which encompasses the NIST certified value bounded by twice its uncertainty $\left(U_{95}\right)$. 


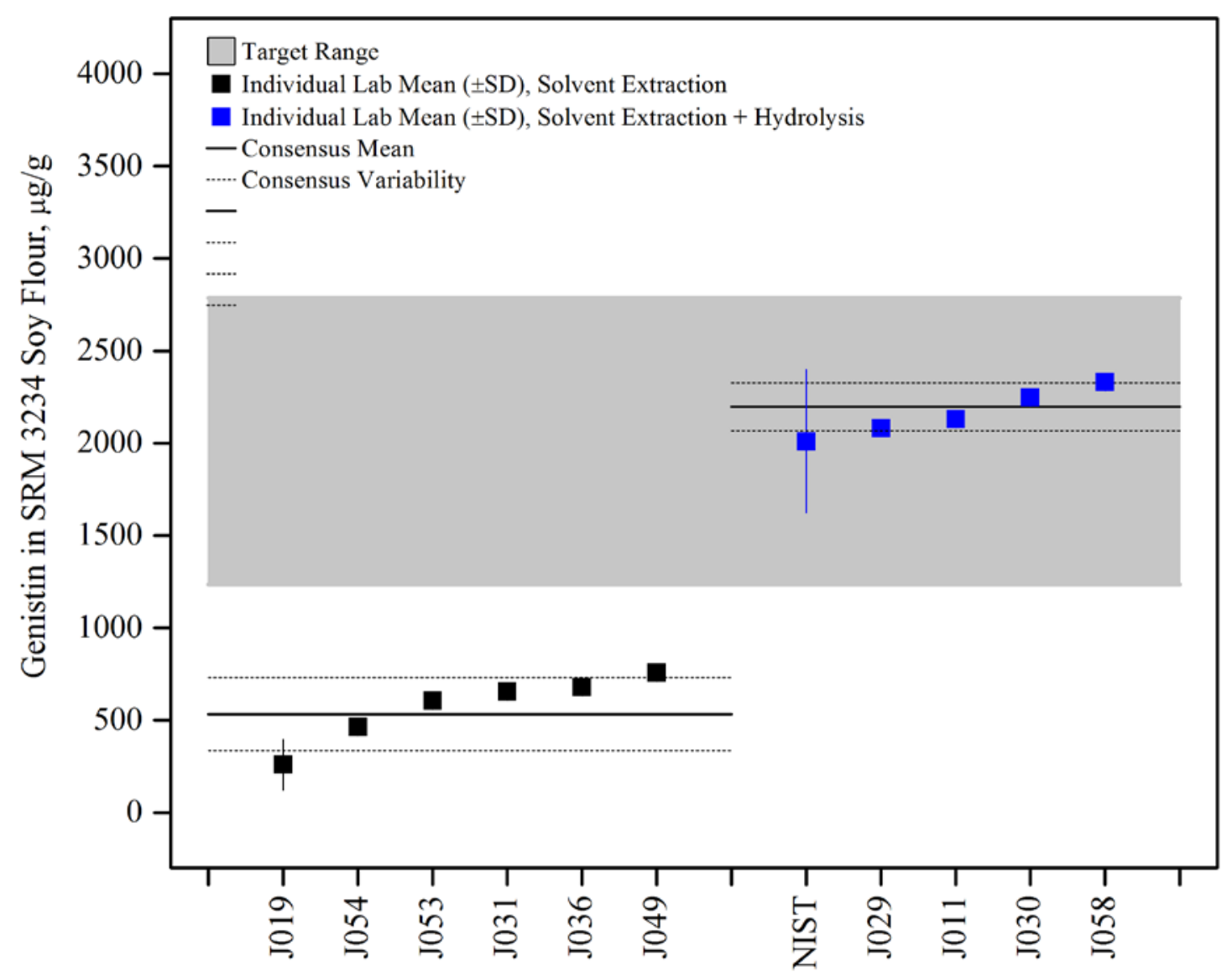

Figure 54. Genistin in SRM 3234 Soy Flour (data summary view, sample preparation comparison). In this view, individual laboratory data are plotted with the individual laboratory standard deviation (error bars). The color of the data point represents the sample preparation method employed. The black solid lines represents the consensus mean for each sample preparation method, and the black dotted lines represent the consensus variability calculated as one standard deviation about the consensus mean for that sample preparation method. The gray shaded region represents the target zone for "acceptable" performance, which encompasses the NIST value determined by LC/abs and ID-LC/MS, bounded by an estimated uncertainty calculated as the standard deviation between the means of values determined by each method. 


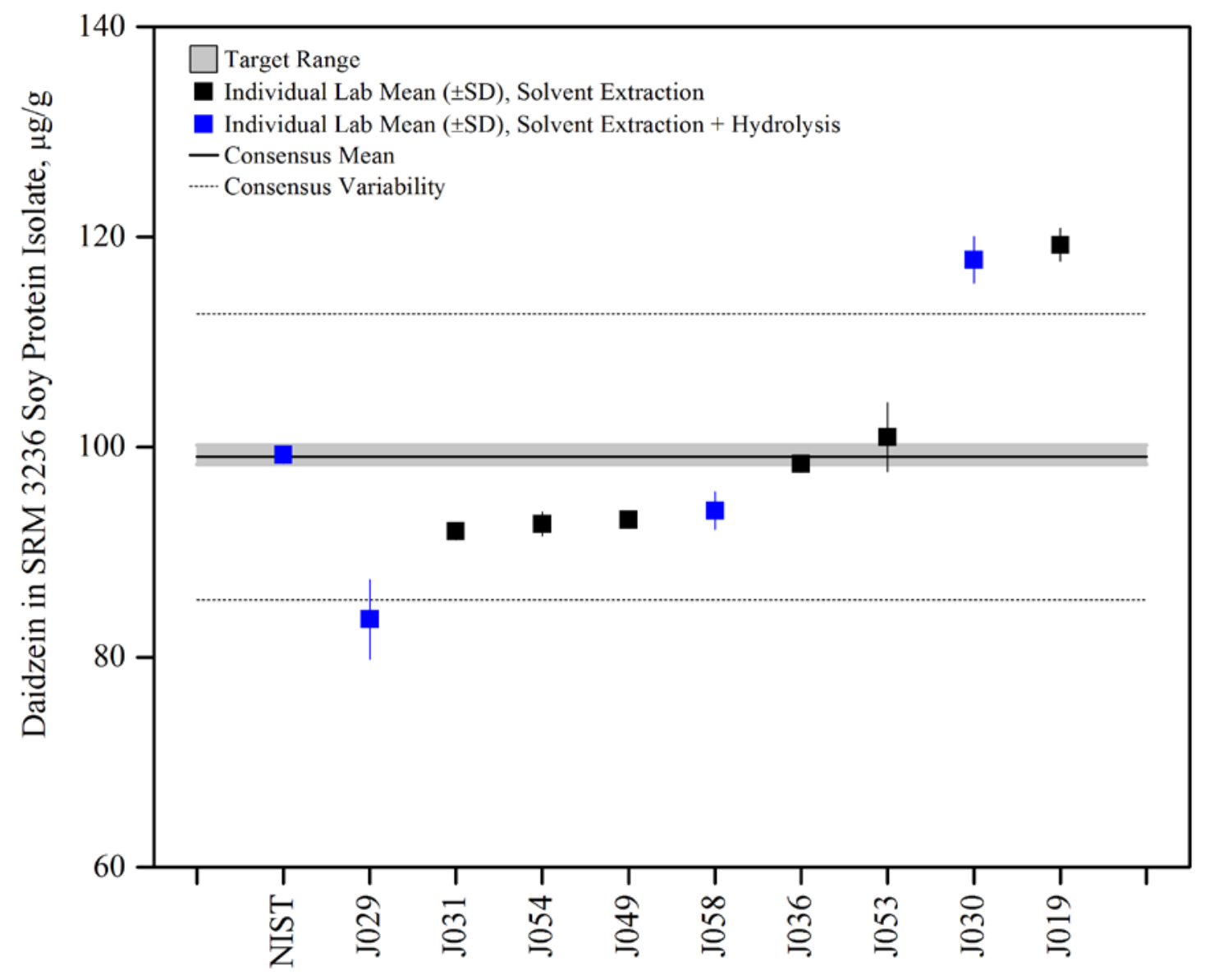

Figure 55. Daidzein in SRM 3236 Soy Protein Isolate (data summary view, sample preparation comparison). In this view, individual laboratory data are plotted with the individual laboratory standard deviation (error bars). The color of the data point represents the sample preparation method employed. The black solid line represents the consensus mean, and the black dotted lines represent the consensus variability calculated as one standard deviation about the consensus mean. The gray shaded region represents the target zone for "acceptable" performance, which encompasses the NIST certified value bounded by twice its uncertainty ( $\left.U_{95}\right)$. 


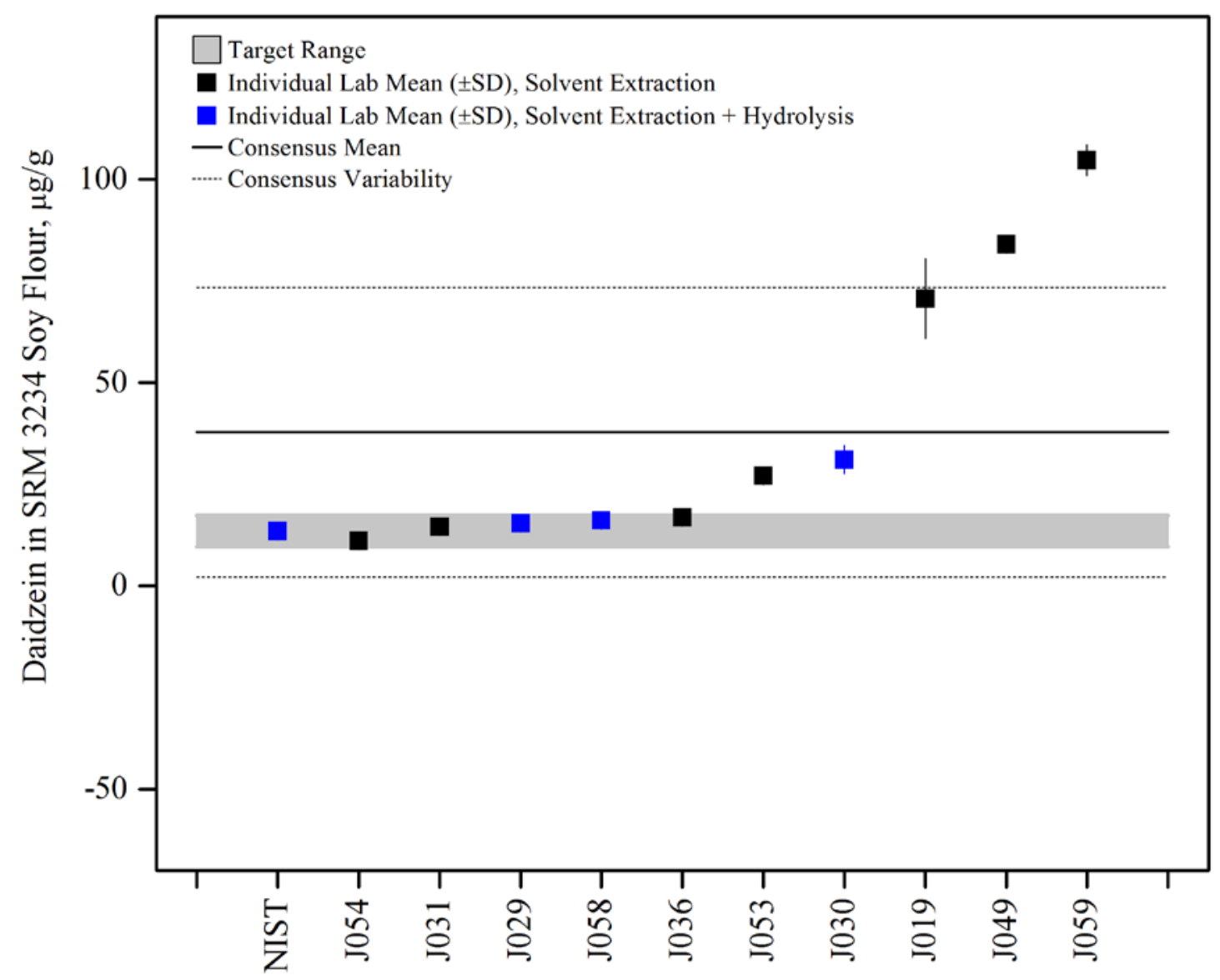

Figure 56. Daidzein in SRM 3234 Soy Flour (data summary view, sample preparation comparison). In this view, individual laboratory data are plotted with the individual laboratory standard deviation (error bars). The color of the data point represents the sample preparation method employed. The black solid line represents the consensus mean, and the black dotted lines represent the consensus variability calculated as one standard deviation about the consensus mean. The gray shaded region represents the target zone for "acceptable” performance, which encompasses the NIST value determined by LC/abs and ID-LC/MS, bounded by an estimated uncertainty calculated as the standard deviation between the means of values determined by each method. 


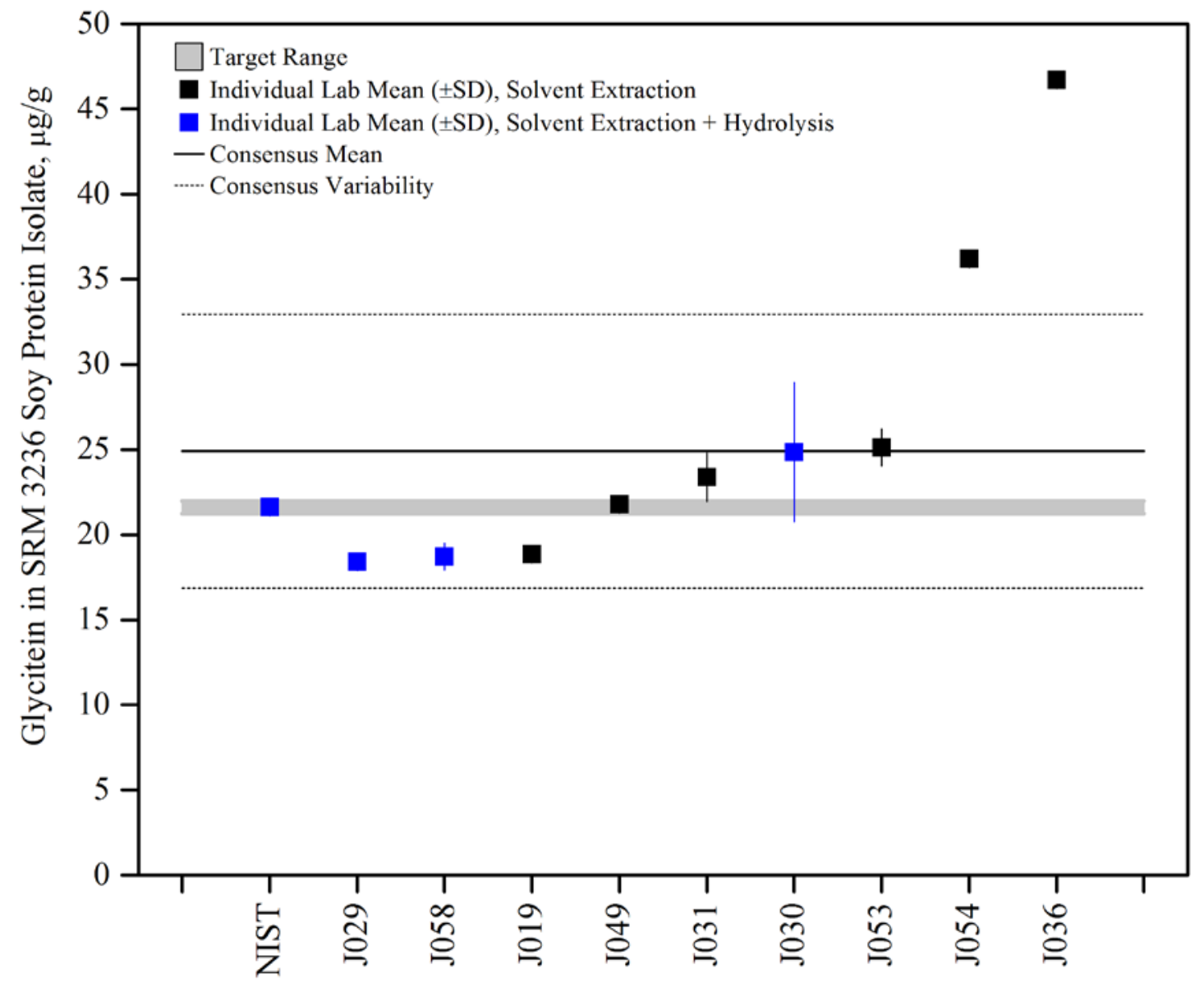

Figure 57. Glycitein in SRM 3236 Soy Protein Isolate (data summary view, sample preparation comparison). In this view, individual laboratory data are plotted with the individual laboratory standard deviation (error bars). The color of the data point represents the sample preparation method employed. The black solid line represents the consensus mean, and the black dotted lines represent the consensus variability calculated as one standard deviation about the consensus mean. The gray shaded region represents the target zone for "acceptable” performance, which encompasses the NIST certified value bounded by twice its uncertainty $\left(U_{95}\right)$. 


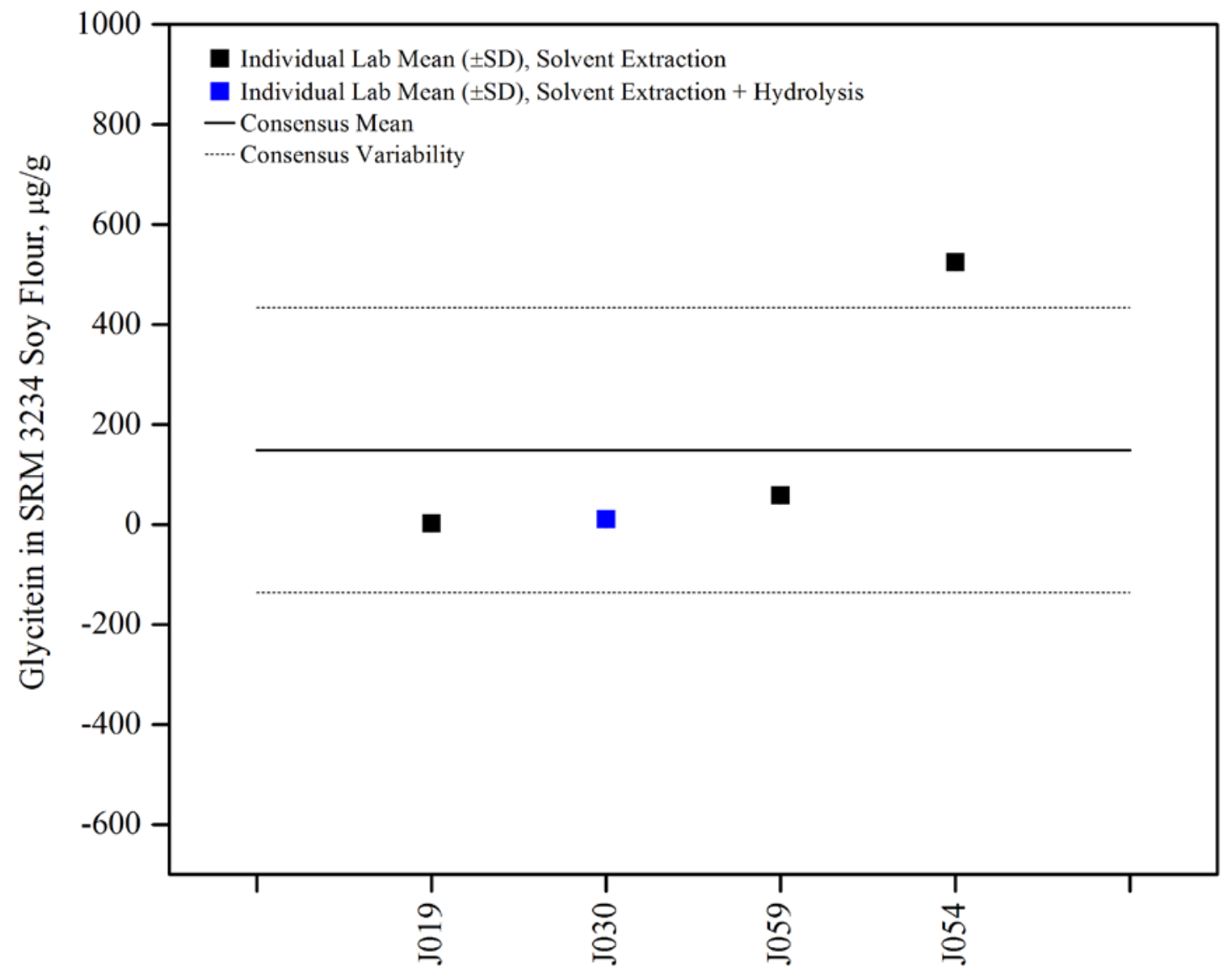

Figure 58. Glycitein in SRM 3234 Soy Flour (data summary view, sample preparation comparison). In this view, individual laboratory data are plotted with the individual laboratory standard deviation (error bars). The color of the data point represents the sample preparation method employed. The black solid line represents the consensus mean, and the black dotted lines represent the consensus variability calculated as one standard deviation about the consensus mean. 


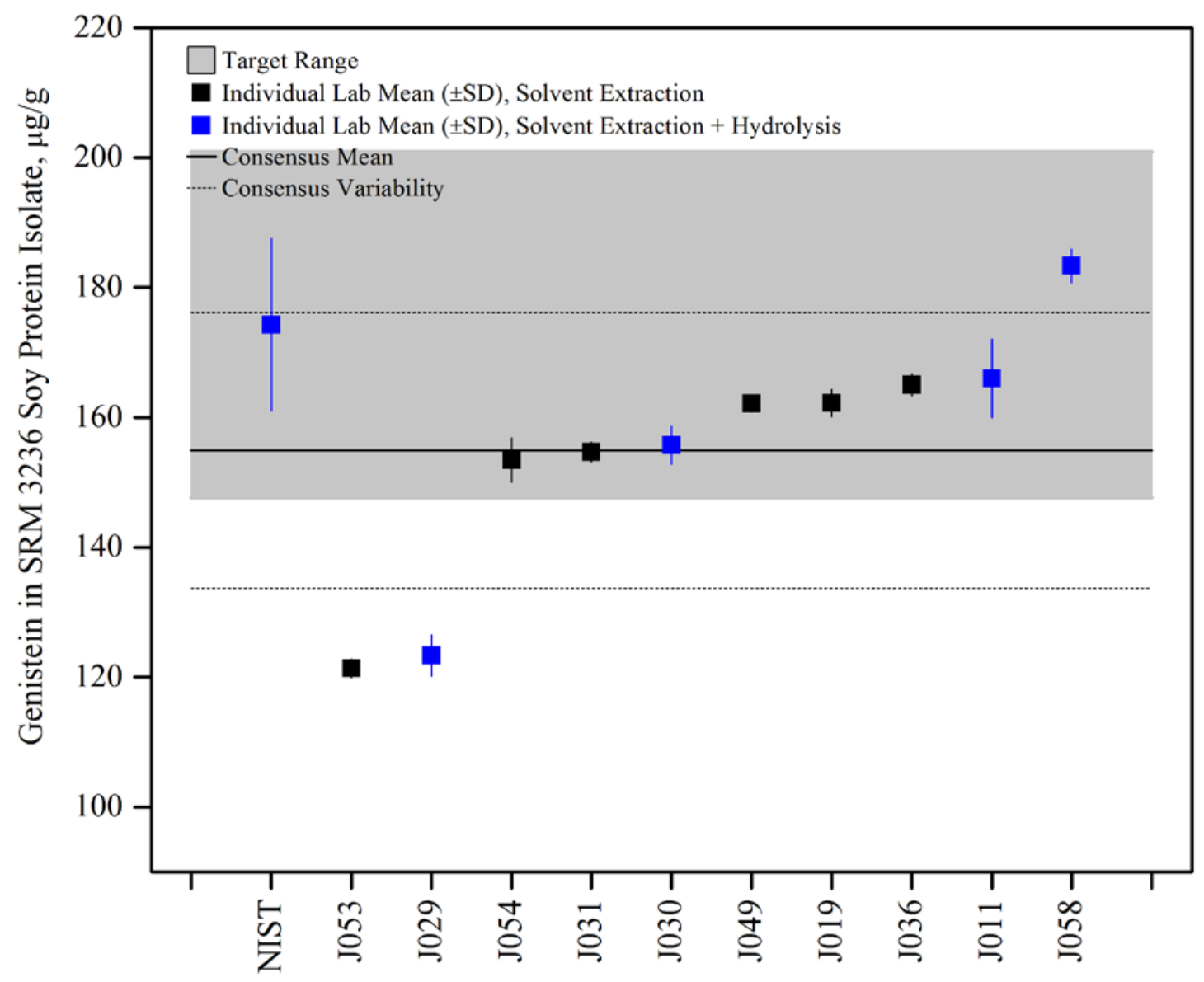

Figure 59. Genistein in SRM 3236 Soy Protein Isolate (data summary view, sample preparation comparison). In this view, individual laboratory data are plotted with the individual laboratory standard deviation (error bars). The color of the data point represents the sample preparation method employed. The black solid line represents the consensus mean, and the black dotted lines represent the consensus variability calculated as one standard deviation about the consensus mean. The gray shaded region represents the target zone for "acceptable” performance, which encompasses the NIST certified value bounded by twice its uncertainty ( $\left.U_{95}\right)$. 


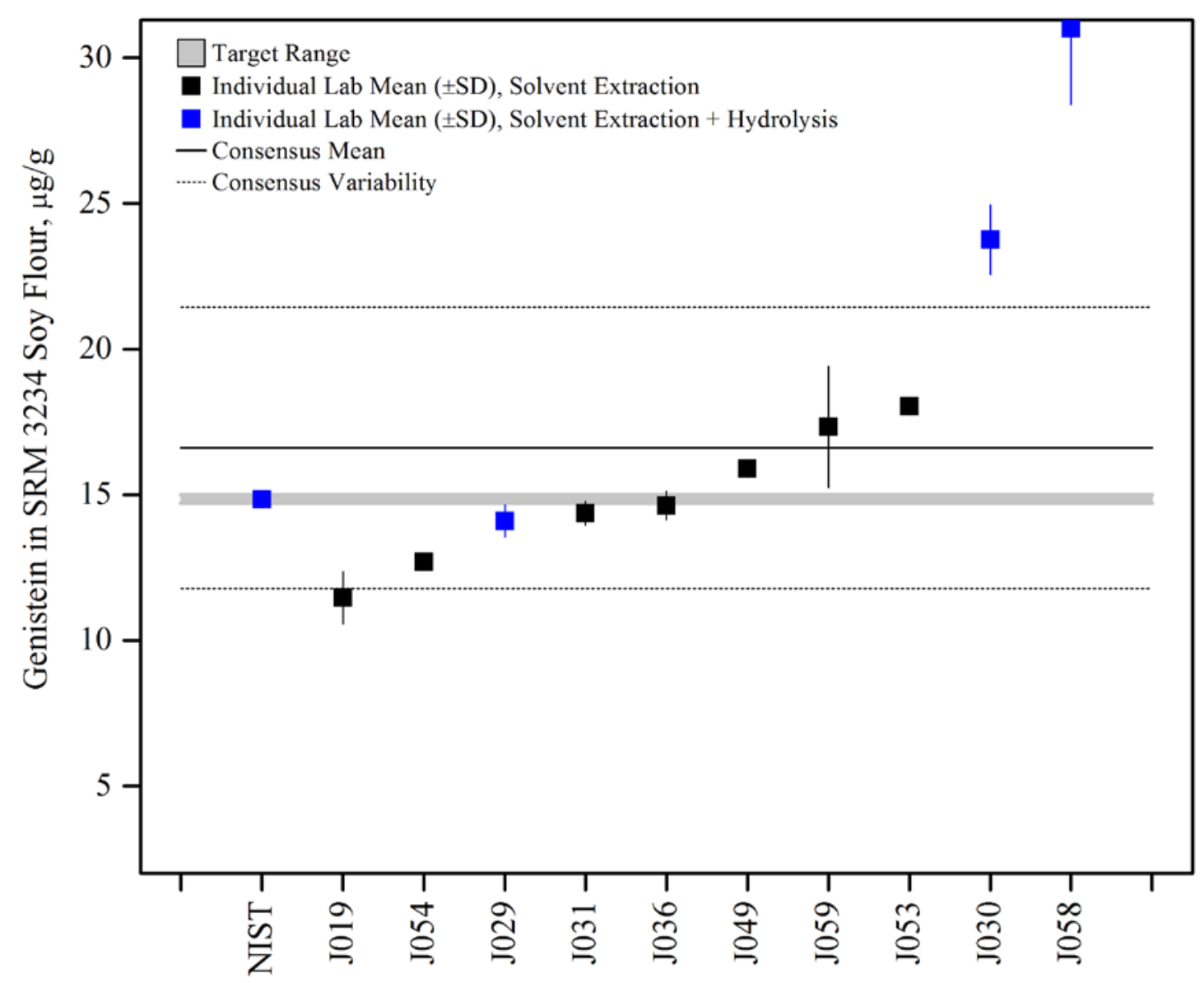

Figure 60. Genistein in SRM 3234 Soy Flour (data summary view, sample preparation comparison). In this view, individual laboratory data are plotted with the individual laboratory standard deviation (error bars). The color of the data point represents the sample preparation method employed. The black solid line represents the consensus mean, and the black dotted lines represent the consensus variability calculated as one standard deviation about the consensus mean. The gray shaded region represents the target zone for "acceptable” performance, which encompasses the NIST value determined by LC/abs and ID-LC/MS, bounded by an estimated uncertainty calculated as the standard deviation between the means of values determined by each method. 


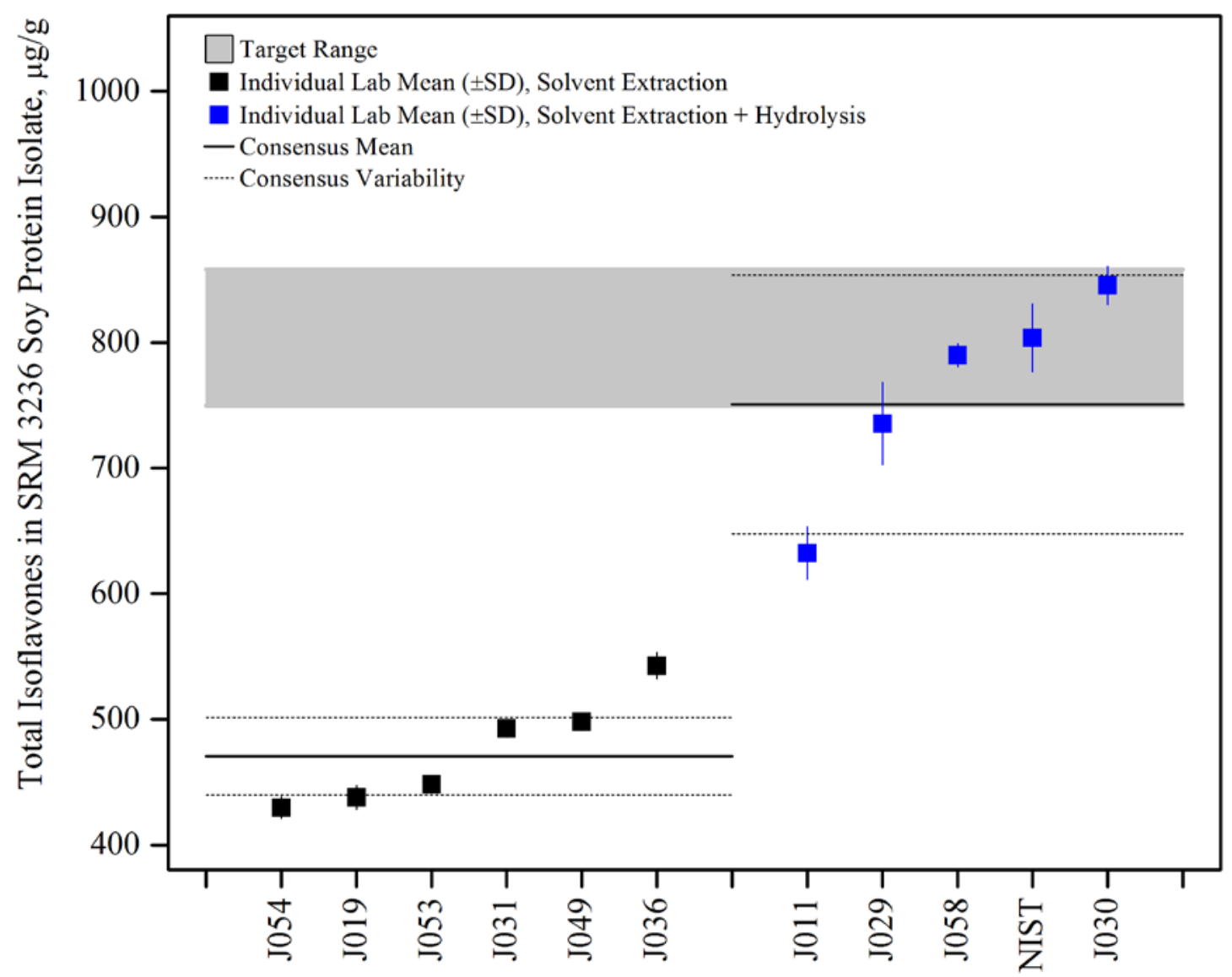

Figure 61. Total isoflavones in SRM 3236 Soy Protein Isolate (data summary view, sample preparation comparison). In this view, individual laboratory data are plotted with the individual laboratory standard deviation (error bars). The color of the data point represents the sample preparation method employed. The black solid lines represents the consensus mean for each sample preparation method, and the black dotted lines represent the consensus variability calculated as one standard deviation about the consensus mean for that sample preparation method. The gray shaded region represents the target zone for "acceptable" performance, which encompasses the NIST certified value bounded by twice its uncertainty $\left(U_{95}\right)$. 


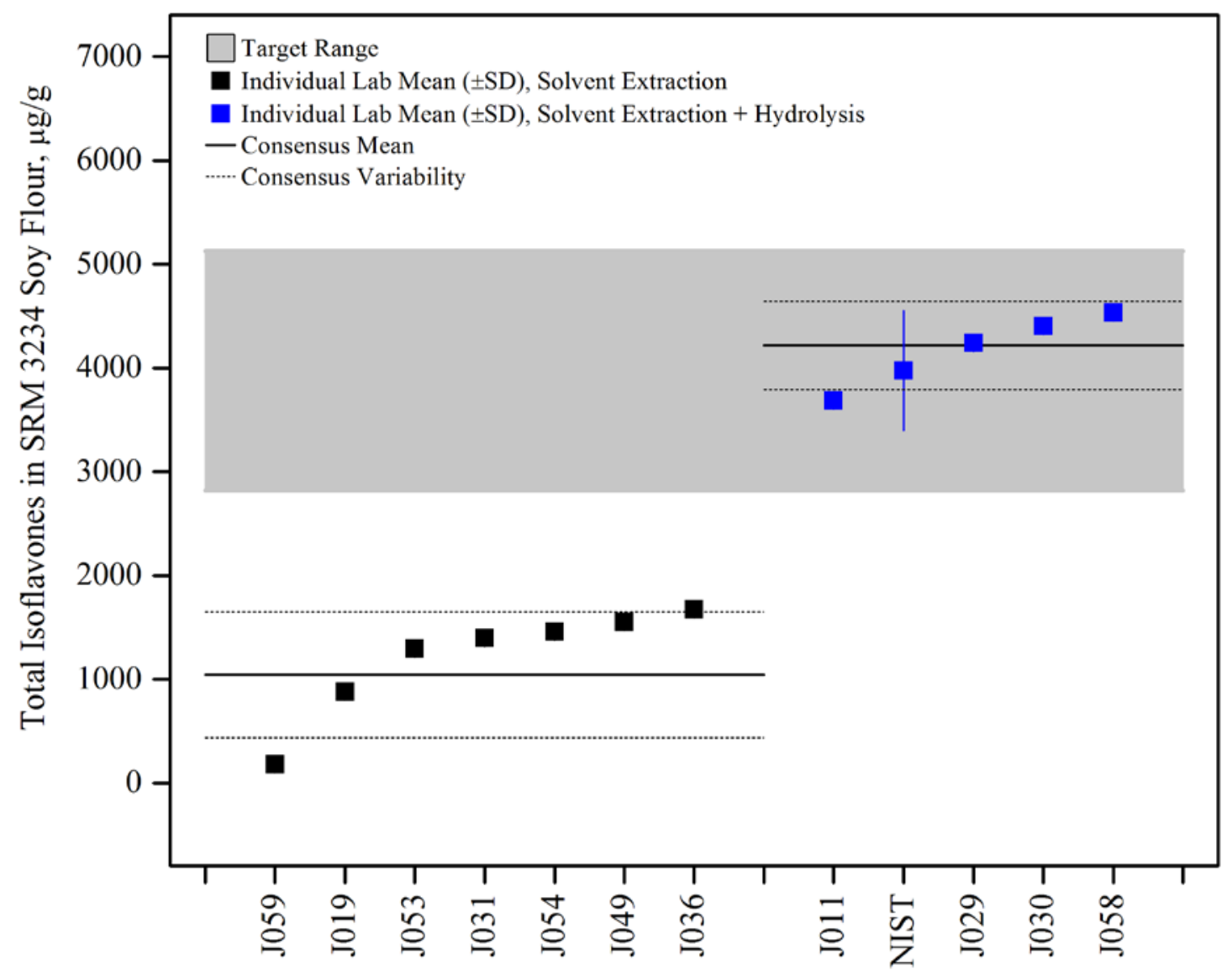

Figure 62. Total isoflavones in SRM 3234 Soy Flour (data summary view, sample preparation comparison). In this view, individual laboratory data are plotted with the individual laboratory standard deviation (error bars). The color of the data point represents the sample preparation method employed. The black solid lines represents the consensus mean for each sample preparation method, and the black dotted lines represent the consensus variability calculated as one standard deviation about the consensus mean for that sample preparation method. The gray shaded region represents the target zone for "acceptable" performance, which encompasses the NIST value determined by LC/abs and ID-LC/MS, bounded by an estimated uncertainty calculated as the standard deviation between the means of values determined by each method. 


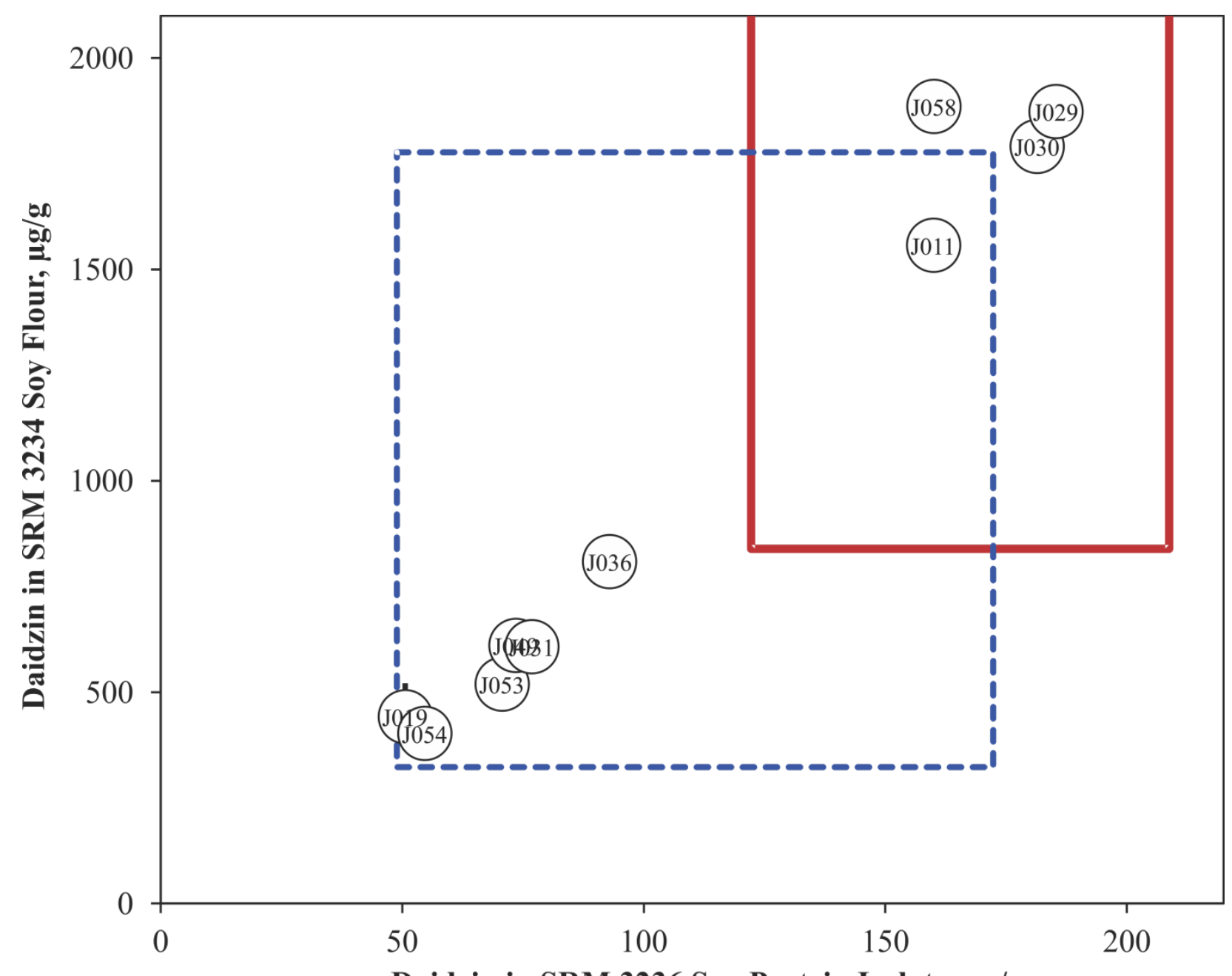

Daidzin in SRM 3236 Soy Protein Isolate, $\mu \mathrm{g} / \mathrm{g}$

Figure 63. Daidzin in SRM 3236 Soy Protein Isolate and SRM 3234 Soy Flour (sample/control comparison view). In this view, the individual laboratory results for the control (soy protein isolate) with a certified value for the analyte are compared to the results for an unknown (soy flour). The solid red box represents the target zone for the control (x-axis) and unknown sample (y-axis). The dotted blue box represents the consensus zone for the control (x-axis) and the unknown sample (y-axis). 


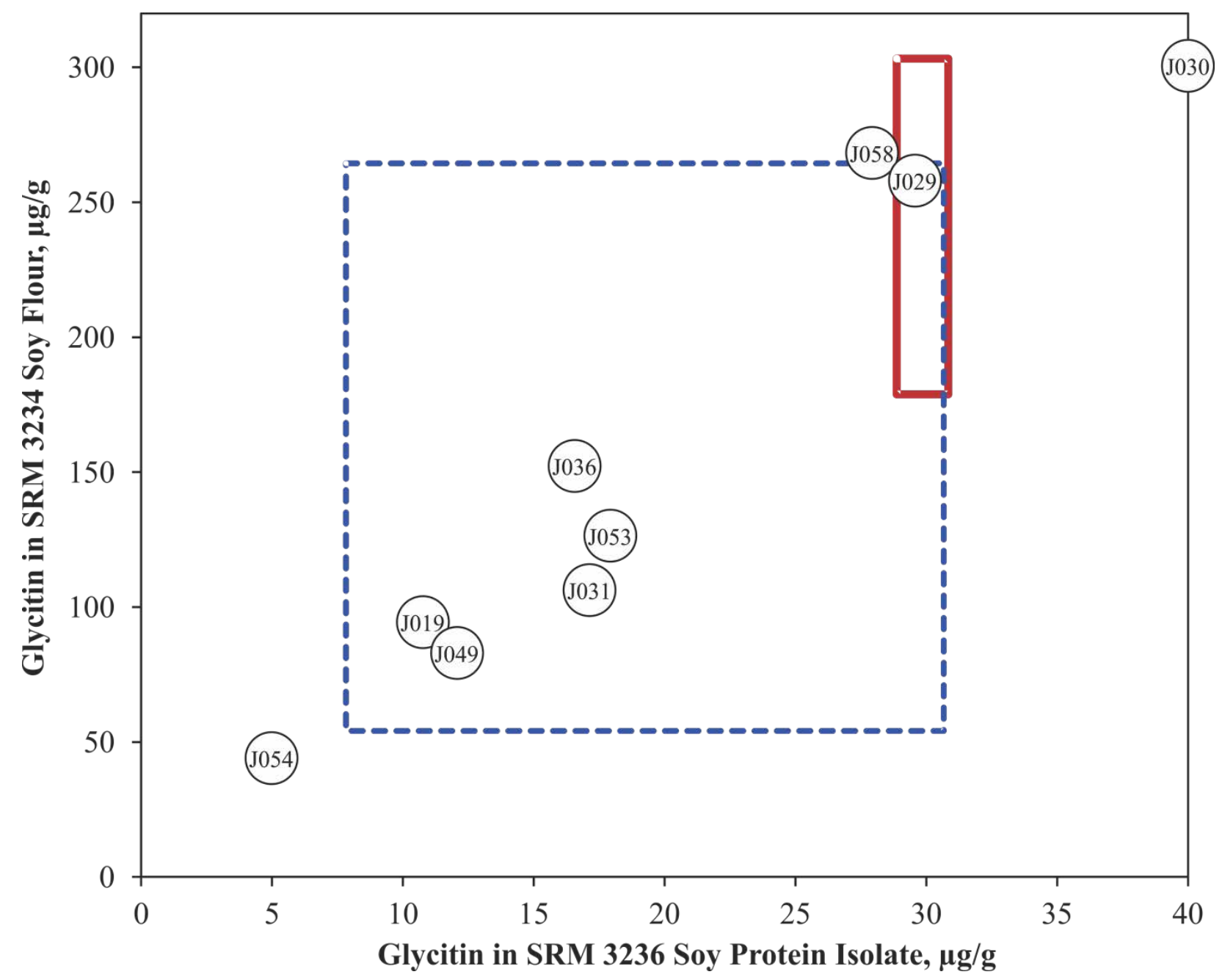

Figure 64. Glycitin in SRM 3236 Soy Protein Isolate and SRM 3234 Soy Flour (sample/control comparison view). In this view, the individual laboratory results for the control (soy protein isolate) with a certified value for the analyte are compared to the results for an unknown (soy flour). The solid red box represents the target zone for the control (x-axis) and unknown sample (y-axis). The dotted blue box represents the consensus zone for the control (x-axis) and the unknown sample (y-axis). 


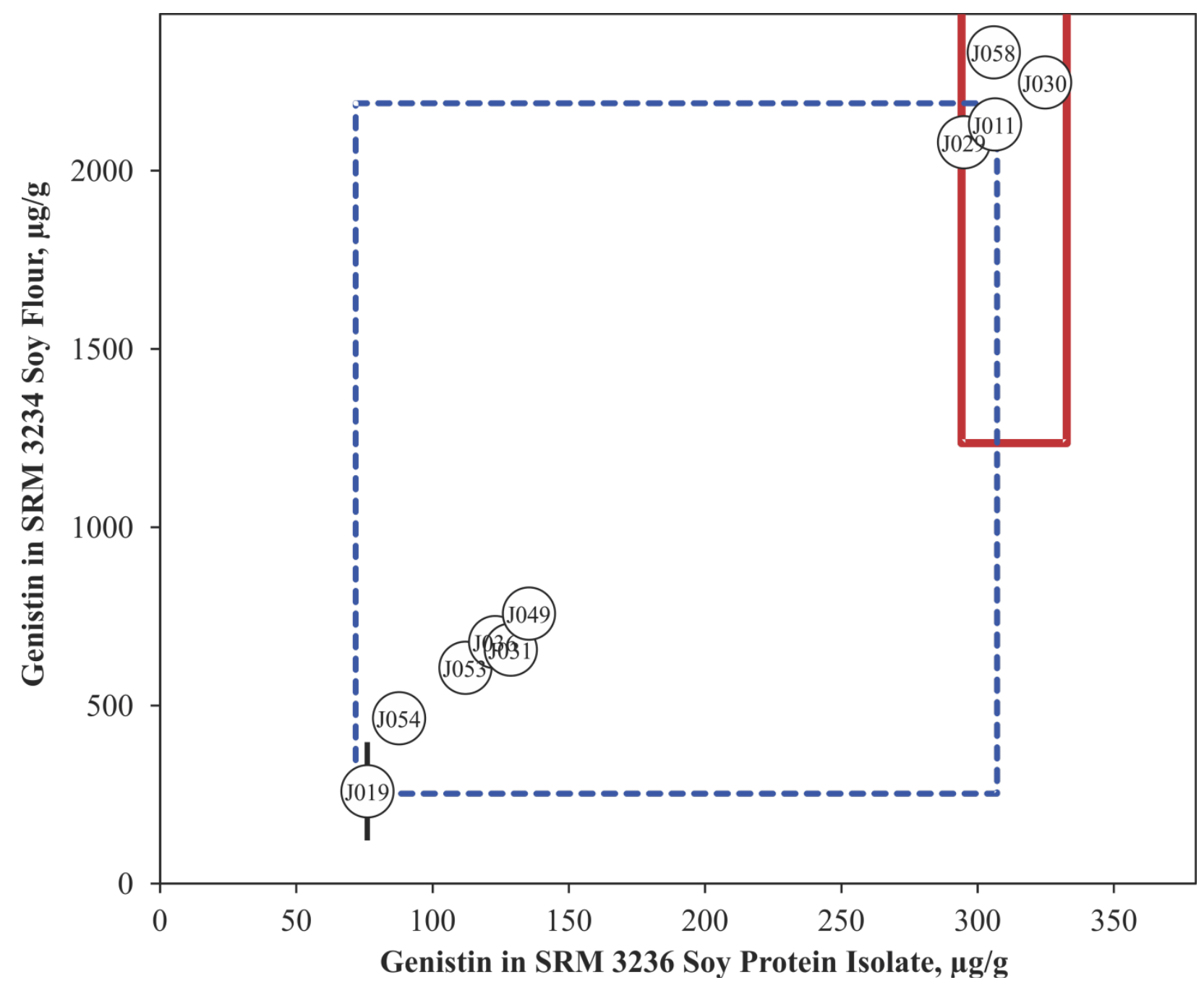

Figure 65. Genistin in SRM 3236 Soy Protein Isolate and SRM 3234 Soy Flour (sample/control comparison view). In this view, the individual laboratory results for the control (soy protein isolate) with a certified value for the analyte are compared to the results for an unknown (soy flour). The solid red box represents the target zone for the control (x-axis) and unknown sample (y-axis). The dotted blue box represents the consensus zone for the control (x-axis) and the unknown sample (y-axis). 


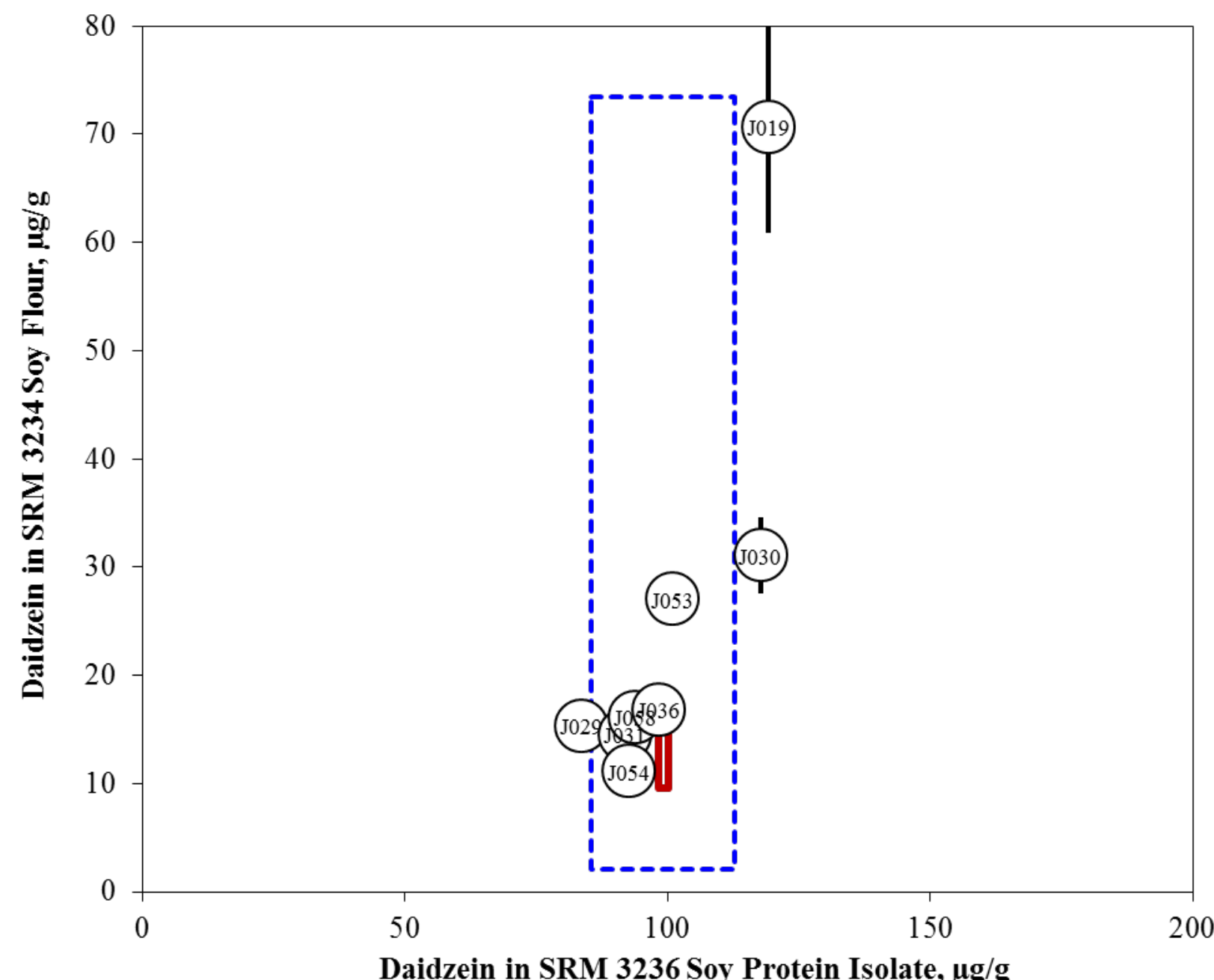

Figure 66. Daidzein in SRM 3236 Soy Protein Isolate and SRM 3234 Soy Flour (sample/control comparison view). In this view, the individual laboratory results for the control (soy protein isolate) with a certified value for the analyte are compared to the results for an unknown (soy flour). The solid red box represents the target zone for the control (x-axis) and unknown sample (y-axis). The dotted blue box represents the consensus zone for the control (x-axis) and the unknown sample (y-axis). 


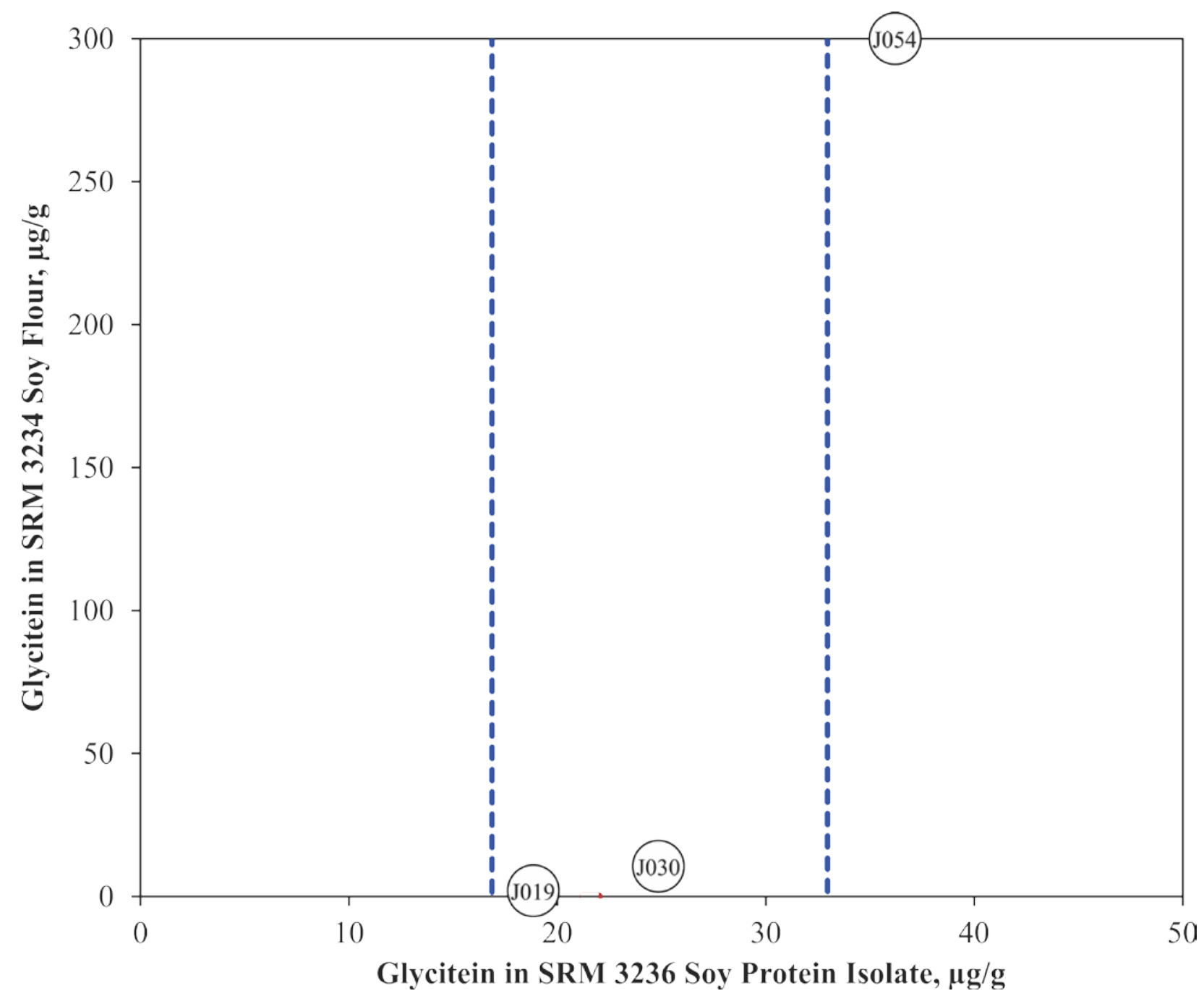

Figure 67. Glycitein in SRM 3236 Soy Protein Isolate and SRM 3234 Soy Flour (sample/control comparison view). In this view, the individual laboratory results for the control (soy protein isolate) with a certified value for the analyte are compared to the results for an unknown (soy flour). The dotted blue box represents the consensus zone for the control (x-axis) and the unknown sample (y-axis). 


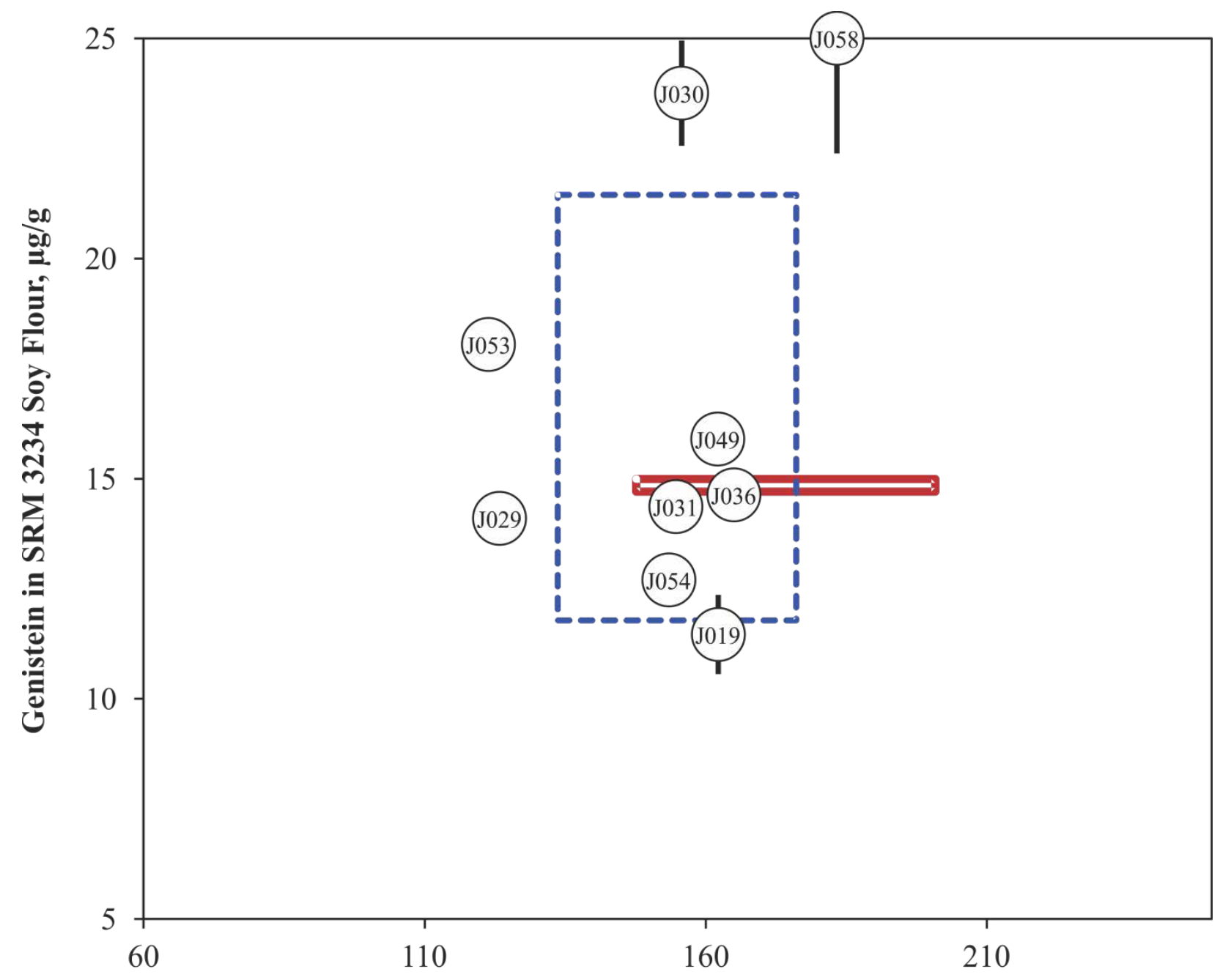

Genistein in SRM 3236 Soy Protein Isolate, $\mu \mathrm{g} / \mathrm{g}$

Figure 68. Genistein in SRM 3236 Soy Protein Isolate and SRM 3234 Soy Flour (sample/control comparison view). In this view, the individual laboratory results for the control (soy protein isolate) with a certified value for the analyte are compared to the results for an unknown (soy flour). The solid red box represents the target zone for the control (x-axis) and unknown sample (y-axis). The dotted blue box represents the consensus zone for the control ( $\mathrm{x}$ axis) and the unknown sample (y-axis). 


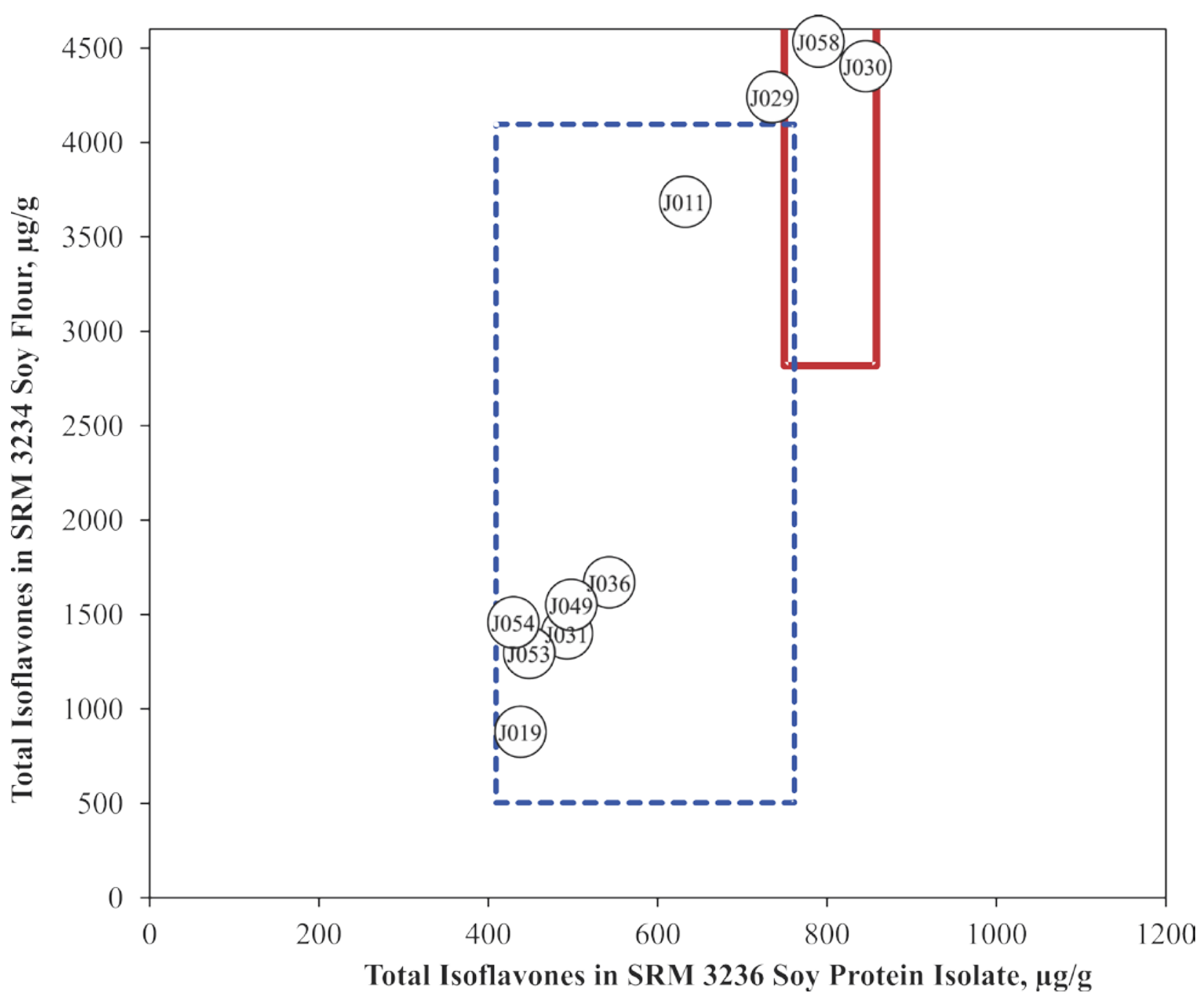

Figure 69. Total isoflavones in SRM 3236 Soy Protein Isolate and SRM 3234 Soy Flour (sample/control comparison view). In this view, the individual laboratory results for the control (soy protein isolate) with a certified value for the analyte are compared to the results for an unknown (soy flour). The solid red box represents the target zone for the control (x-axis) and unknown sample (y-axis). The dotted blue box represents the consensus zone for the control (xaxis) and the unknown sample (y-axis). 


\section{BOTANICAL IDENTITY OF PURE AND ADULTERATED GINKGO BILOBA}

\section{Study Overview}

In this study, ten vials labeled Ginkgo biloba extract and ten vials labeled Ginkgo biloba leaf were provided to the participants.

\section{Sample Information}

Gingko biloba Extract. Participants were provided with ten vials labeled Ginkgo biloba extract. Before use, participants were instructed to mix each vial thoroughly. Participants were asked to report “yes” for vials identified as Ginkgo biloba. If possible, reporting of the mass fraction (percentage) of Ginkgo biloba was requested. Participants were asked to report "no" for vials identified as not Ginkgo biloba. If possible, participants were asked to indicate the main constituent of the sample.

Gingko biloba Leaf. Participants were provided with ten vials labeled Ginkgo biloba leaf. Before use, participants were instructed to mix each vial thoroughly. Participants were asked to report “yes” for vials identified as Ginkgo biloba. If possible, reporting of the mass fraction (percentage) of Ginkgo biloba was requested. Participants were asked to report "no" for vials identified as not Ginkgo biloba. If possible, participants were asked to indicate the main constituent of the sample.

\section{Study Results}

- Twenty-five laboratories enrolled in this exercise and received samples. Nineteen laboratories reported results for some portion of the study (76\% participation).

- The Gingko biloba extract samples were adulterated with $0 \%, 10 \%, 25 \%, 50 \%$, or $75 \%$ green tea extract and $0 \%, 10 \%, 25 \%, 50 \%$, or $75 \%$ of microcellulose filler.

- Six laboratories attempted to identify the mass percentage of Gingko biloba extract in each sample.

- The Gingko biloba leaf samples were adulterated with $0 \%, 10 \%, 25 \%, 50 \%$, or $75 \%$ green tea leaves and $0 \%, 10 \%, 25 \%, 50 \%$, or $75 \%$ of microcellulose filler.

- Five laboratories attempted to identify the mass percentage of Gingko biloba leaves in each sample.

- Laboratories that used only thin-layer chromatography (TLC) for identity and detection of adulteration were better able to detect adulteration with other plants than with the microcellulose, which added no bands to the chromatogram.

- Laboratories were able to detect adulteration with green tea at lower levels in the plant material than in the extract material.

- Detection of adulteration with microcellulose was approximately the same in the plant material and the extract material.

\section{Technical Recommendations}

The following recommendations are based on results obtained by the participants in this study.

- TLC is a good screening method for identity and for finding adulteration, provided the adulterant has characteristic chromatographic bands that are different from those of the plant material under investigation.

- Laboratories that performed multiple methods (e.g., TLC and microscopy) were able to provide quantitative results for the percent adulteration as well as information as to whether or not the sample was an adulterated Ginkgo product. 
- In future studies, more specific questions will be asked about testing methods.

- Laboratories will be given specific instructions on whether to test for authenticity/identity or adulteration. 
Table 33. Individual data summary table (NIST) for botanical identity of pure and adulterated Ginkgo biloba.

\section{National Institute of Standards \& Technology}

\begin{tabular}{ccc} 
& Lab Code: & NIST \\
\hline & Sample & Adulterant \\
\hline L8 & Ginkgo Leaves & -- \\
L5 & Ginkgo Leaves & Green Tea Leaves \\
L9 & Ginkgo Leaves & Green Tea Leaves \\
L2 & Ginkgo Leaves & Green Tea Leaves \\
L6 & Ginkgo Leaves & Green Tea Leaves \\
\hline L4 & Ginkgo Leaves & -- \\
L1 & Ginkgo Leaves & Microcellulose \\
L7 & Ginkgo Leaves & Microcellulose \\
L3 & Ginkgo Leaves & Microcellulose \\
L10 & Ginkgo Leaves & Microcellulose \\
\hline E5 & Ginkgo Extract & -- \\
E1 & Ginkgo Extract & Green Tea Extract \\
E2 & Ginkgo Extract & Green Tea Extract \\
E4 & Ginkgo Extract & Green Tea Extract \\
E9 & Ginkgo Extract & Green Tea Extract \\
\hline E7 & Ginkgo Extract & -- \\
E8 & Ginkgo Extract & Microcellulose \\
E3 & Ginkgo Extract & Microcellulose \\
E6 & Ginkgo Extract & Microcellulose \\
E10 & Ginkgo Extract & Microcellulose \\
\hline & &
\end{tabular}

\begin{tabular}{cc} 
1. Your Results \\
\hline Y/N & $\%$ \\
\hline Yes & $100 \%$ \\
Yes & $90 \%$ \\
Yes & $75 \%$ \\
Yes & $50 \%$ \\
Yes & $25 \%$ \\
\hline Yes & $100 \%$ \\
Yes & $90 \%$ \\
Yes & $75 \%$ \\
Yes & $50 \%$ \\
Yes & $25 \%$ \\
\hline Yes & $100 \%$ \\
Yes & $90 \%$ \\
Yes & $75 \%$ \\
Yes & $50 \%$ \\
Yes & $25 \%$ \\
\hline Yes & $100 \%$ \\
Yes & $90 \%$ \\
Yes & $75 \%$ \\
Yes & $50 \%$ \\
Yes & $25 \%$ \\
\hline
\end{tabular}

\begin{tabular}{ccc} 
& \multicolumn{2}{c}{ 2. Commun } \\
\hline $\mathrm{N}_{\mathrm{Y} / \mathrm{N}}$ & $\mathrm{N}_{\mathrm{Yes}}$ & $\mathrm{N}_{\mathrm{No}}$ \\
\hline 19 & 17 & 2 \\
19 & 9 & 10 \\
19 & 7 & 12 \\
19 & 7 & 12 \\
19 & 8 & 11 \\
\hline 19 & 17 & 2 \\
19 & 16 & 3 \\
19 & 16 & 3 \\
19 & 15 & 4 \\
19 & 15 & 4 \\
\hline 18 & 16 & 2 \\
18 & 14 & 4 \\
17 & 5 & 12 \\
17 & 6 & 11 \\
17 & 7 & 10 \\
\hline 18 & 17 & 1 \\
18 & 7 & 11 \\
17 & 14 & 3 \\
17 & 12 & 5 \\
17 & 12 & 5 \\
\hline & &
\end{tabular}

\begin{tabular}{ccc} 
Results & & \\
$\mathrm{N}_{\%}$ & Avg & $\mathrm{SD}$ \\
\hline 3 & $98 \%$ & $3 \%$ \\
5 & $82 \%$ & $6 \%$ \\
4 & $69 \%$ & $3 \%$ \\
5 & $58 \%$ & $29 \%$ \\
5 & $28 \%$ & $6 \%$ \\
\hline 4 & $84 \%$ & $33 \%$ \\
4 & $89 \%$ & $5 \%$ \\
3 & $77 \%$ & $3 \%$ \\
4 & $53 \%$ & $5 \%$ \\
3 & $18 \%$ & $8 \%$ \\
\hline 4 & $90 \%$ & $20 \%$ \\
4 & $85 \%$ & $7 \%$ \\
5 & $62 \%$ & $15 \%$ \\
6 & $38 \%$ & $26 \%$ \\
3 & $28 \%$ & $41 \%$ \\
\hline 4 & $98 \%$ & $3 \%$ \\
4 & $68 \%$ & $22 \%$ \\
4 & $73 \%$ & $13 \%$ \\
4 & $43 \%$ & $10 \%$ \\
2 & $10 \%$ & $0 \%$ \\
\hline & &
\end{tabular}

\begin{tabular}{cc}
\multicolumn{2}{c}{ 3. Target } \\
\hline Y/N $\mathrm{N}_{\mathrm{NIST}}$ & $\%_{\mathrm{NIST}}$ \\
\hline Yes & $100 \%$ \\
Yes & $90 \%$ \\
Yes & $75 \%$ \\
Yes & $50 \%$ \\
Yes & $25 \%$ \\
\hline Yes & $100 \%$ \\
Yes & $90 \%$ \\
Yes & $75 \%$ \\
Yes & $50 \%$ \\
Yes & $25 \%$ \\
\hline Yes & $100 \%$ \\
Yes & $90 \%$ \\
Yes & $75 \%$ \\
Yes & $50 \%$ \\
Yes & $25 \%$ \\
\hline Yes & $100 \%$ \\
Yes & $90 \%$ \\
Yes & $75 \%$ \\
Yes & $50 \%$ \\
Yes & $25 \%$ \\
\hline
\end{tabular}


Table 34. Data summary table for Ginkgo biloba extracts (yes/no).

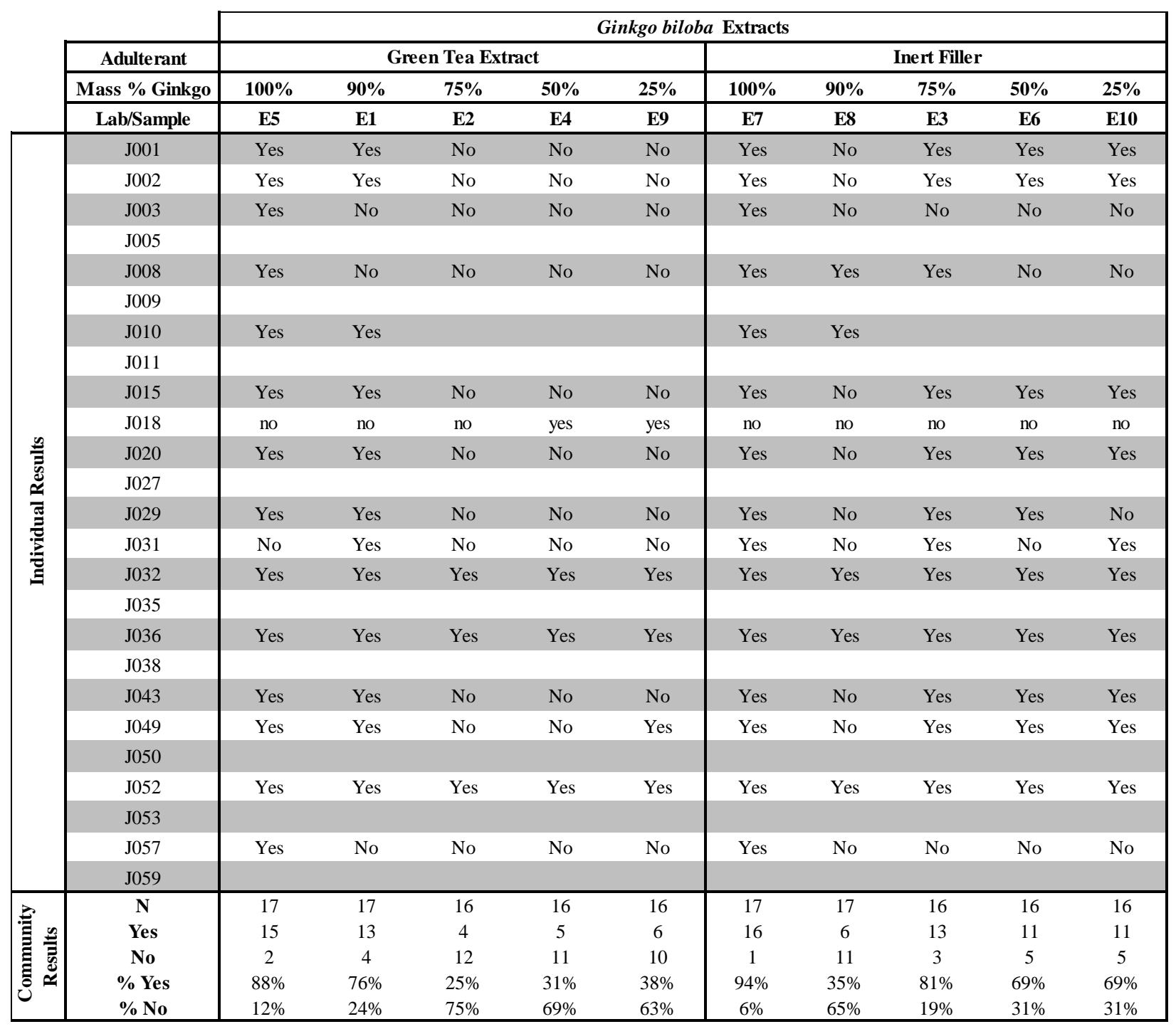


Table 35. Data summary table for Ginkgo biloba extracts (mass percentage).

\begin{tabular}{|c|c|c|c|c|c|c|c|c|c|c|c|}
\hline & \multirow{3}{*}{\begin{tabular}{c|} 
Adulterant \\
Mass \% Ginkgo
\end{tabular}} & \\
\hline & & \multicolumn{10}{|c|}{ Ginkgo biloba Extracts } \\
\hline & & $100 \%$ & $90 \%$ & $75 \%$ & $50 \%$ & $25 \%$ & $100 \%$ & $90 \%$ & $75 \%$ & $50 \%$ & $25 \%$ \\
\hline & Lab/Sample & E5 & E1 & E2 & E4 & E9 & E7 & E8 & E3 & E6 & E10 \\
\hline \multirow{25}{*}{ 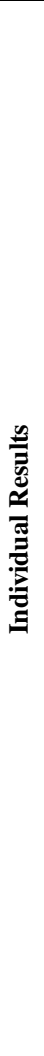 } & J001 & & & & & & & & & & \\
\hline & J002 & & & & & & & & & & \\
\hline & J003 & $100 \%$ & $80 \%$ & $60 \%$ & $30 \%$ & $10 \%$ & $100 \%$ & $77 \%$ & $60 \%$ & $30 \%$ & $10 \%$ \\
\hline & J005 & & & & & & & & & & \\
\hline & J008 & & & & & & & & & & \\
\hline & J009 & & & & & & & & & & \\
\hline & J010 & & & & & & & & & & \\
\hline & J011 & & & & & & & & & & \\
\hline & J015 & & & & & & & & & & \\
\hline & J018 & & & & $0.10 \%$ & $0.08 \%$ & & & & & \\
\hline & J020 & & & & & & & & & & \\
\hline & J027 & & & & & & & & & & \\
\hline & J029 & & & & & & & & & & \\
\hline & J031 & $60 \%$ & $80 \%$ & $50 \%$ & $70 \%$ & & & & $90 \%$ & $50 \%$ & \\
\hline & J032 & $100 \%$ & $85 \%$ & $65 \%$ & $35 \%$ & $10-15 \%$ & $95 \%$ & $80 \%$ & $65 \%$ & $40 \%$ & $10-15 \%$ \\
\hline & J035 & & & & & & & & & & \\
\hline & J036 & & & & & & & & & & \\
\hline & J038 & & & & & & & & & & \\
\hline & $\mathrm{J} 043$ & & & & & & & & & & \\
\hline & J049 & & & & & & & & & & \\
\hline & $\mathrm{J} 050$ & & & & & & & & & & \\
\hline & J052 & $12 \%$ & $10 \%$ & $8 \%$ & $5 \%$ & $2 \%$ & $11 \%$ & $10 \%$ & $8 \%$ & $5 \%$ & $1 \%$ \\
\hline & J053 & & & & & & & & & & \\
\hline & J057 & $100 \%$ & $95 \%$ & $50 \%$ & $25 \%$ & $75 \%$ & $100 \%$ & $80 \%$ & $75 \%$ & $50 \%$ & $10 \%$ \\
\hline & J059 & & & & & & & & & & \\
\hline
\end{tabular}


Table 36. Data summary table for Ginkgo biloba leaves (yes/no).

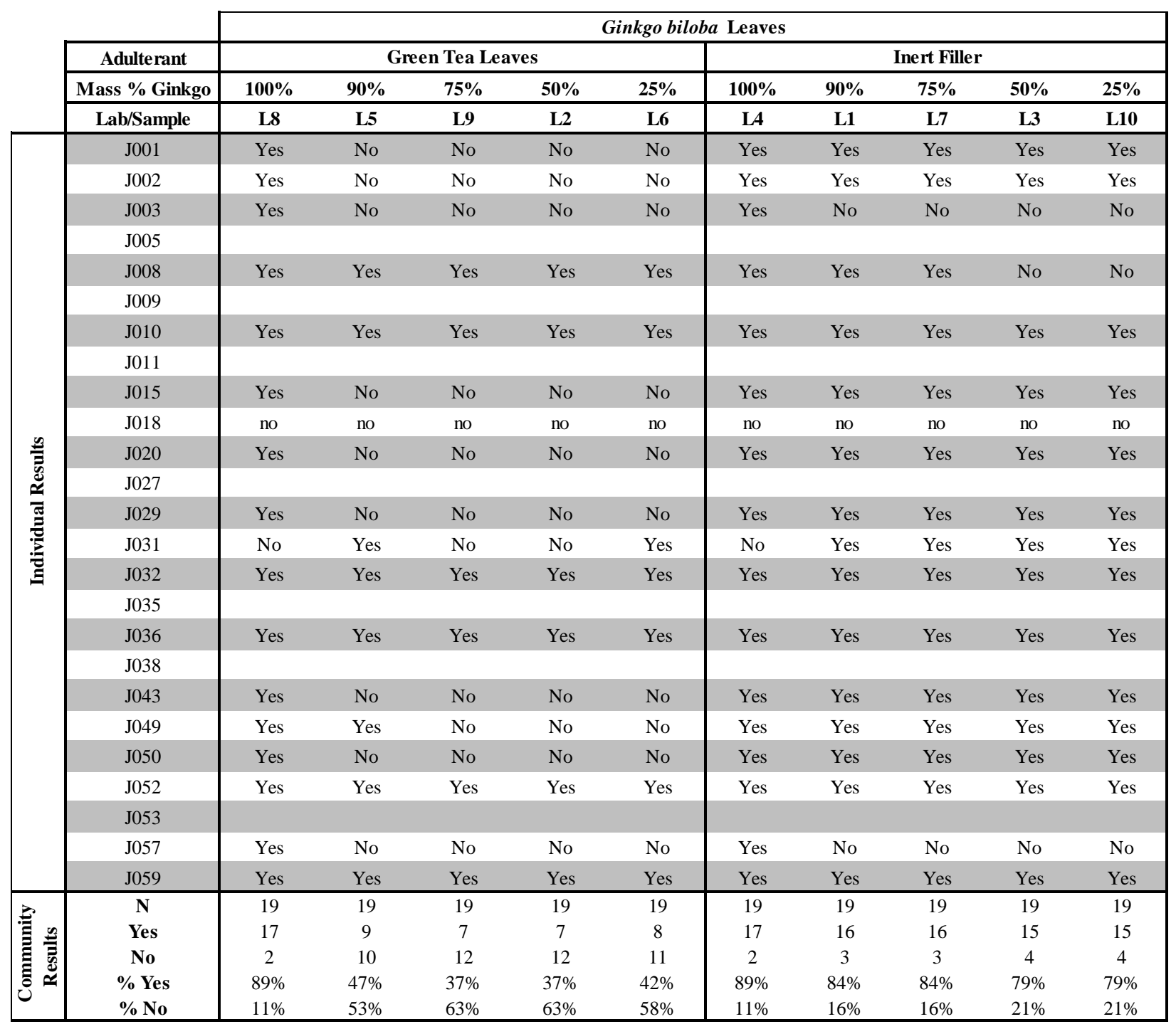


Table 37. Data summary table for Ginkgo biloba leaves (mass percentage).

\begin{tabular}{|c|c|c|c|c|c|c|c|c|c|c|c|}
\hline & \multirow{3}{*}{\begin{tabular}{c|} 
Adulterant \\
Mass \% Ginkgo
\end{tabular}} & \multicolumn{10}{|c|}{ Ginkgo biloba Leaves } \\
\hline & & \multicolumn{5}{|c|}{ Green Tea Leaves } & \multicolumn{5}{|c|}{ Inert Filler } \\
\hline & & $100 \%$ & $90 \%$ & $75 \%$ & $50 \%$ & $25 \%$ & $100 \%$ & $90 \%$ & $75 \%$ & $50 \%$ & $25 \%$ \\
\hline & Lab/Sample & L8 & L5 & L9 & $\mathbf{L 2}$ & L6 & L4 & L1 & L7 & L3 & L10 \\
\hline \multirow{25}{*}{ 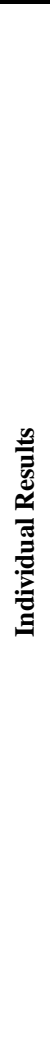 } & J001 & & & & & & & & & & \\
\hline & J002 & & & & & & & & & & \\
\hline & J003 & $100 \%$ & $80 \%$ & $70 \%$ & $40 \%$ & $30 \%$ & $100 \%$ & $85 \%$ & $75 \%$ & $50 \%$ & $20 \%$ \\
\hline & J005 & & & & & & & & & & \\
\hline & J008 & & & & & & & & & & \\
\hline & J009 & & & & & & & & & & \\
\hline & $\mathrm{J} 010$ & & & & & & & & & & \\
\hline & J011 & & & & & & & & & & \\
\hline & J015 & & & & & & & & & & \\
\hline & J018 & & & & & & & & & & \\
\hline & J020 & & & & & & & & & & \\
\hline & J027 & & & & & & & & & & \\
\hline & J029 & & & & & & & & & & \\
\hline & J031 & & $75 \%$ & & $110 \%$ & $35 \%$ & $35 \%$ & $85 \%$ & & $60 \%$ & \\
\hline & J032 & $95 \%$ & $85 \%$ & $65 \%$ & $45 \%$ & $20 \%$ & $100 \%$ & $92 \%$ & $75 \%$ & $50 \%$ & $25 \%$ \\
\hline & J035 & & & & & & & & & & \\
\hline & J036 & & & & & & & & & & \\
\hline & J038 & & & & & & & & & & \\
\hline & J043 & & & & & & & & & & \\
\hline & J049 & & & & & & & & & & \\
\hline & $\mathrm{J} 050$ & & & & & & & & & & \\
\hline & J052 & $0.68 \%$ & $0.68 \%$ & $0.76 \%$ & $0.59 \%$ & $0.53 \%$ & $0.69 \%$ & $0.66 \%$ & $0.50 \%$ & $0.34 \%$ & $0.28 \%$ \\
\hline & J053 & & & & & & & & & & \\
\hline & J057 & $100 \%$ & $80 \%$ & $70 \%$ & $50 \%$ & $25 \%$ & $100 \%$ & $95 \%$ & $80 \%$ & $50 \%$ & $10 \%$ \\
\hline & J059 & & & & & & & & & & \\
\hline
\end{tabular}




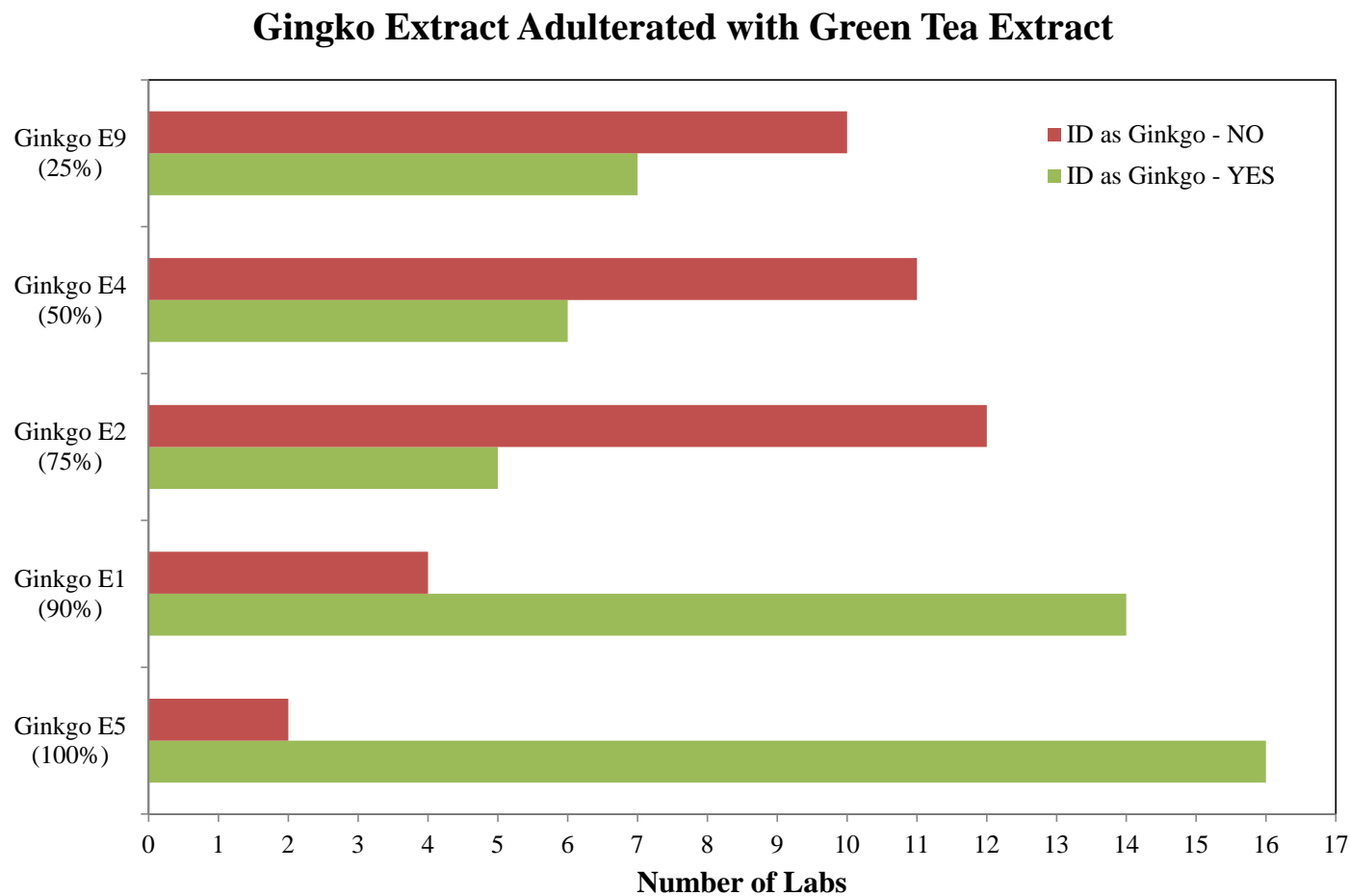

\section{Gingko Extract Adulterated with Cellulose}

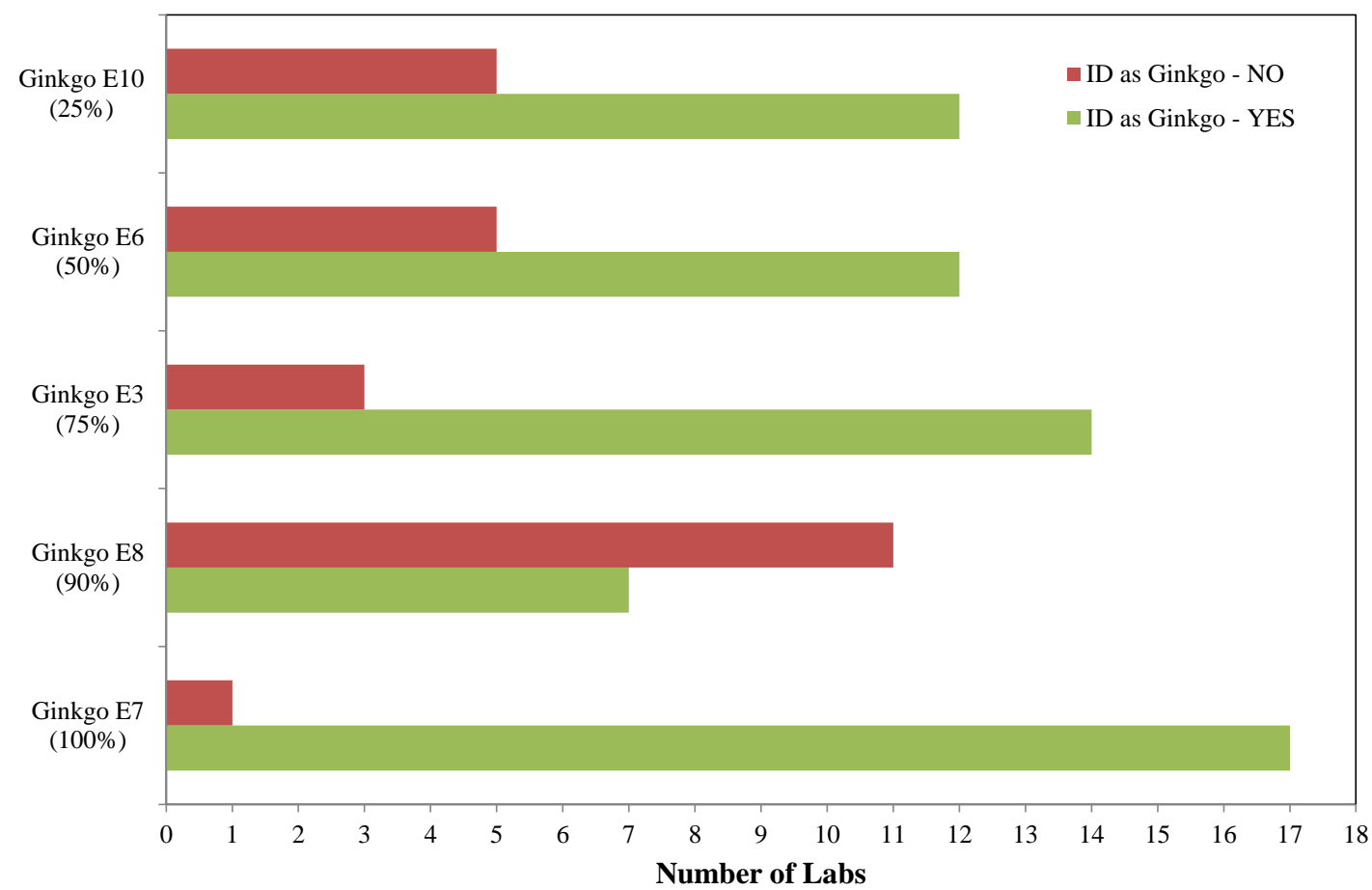

Figure 70. Adulterated Ginkgo biloba extract. These two charts show the number of laboratories reporting Ginkgo extract as being adulterated. The samples in the top chart were adulterated with varying amounts of green tea extract. The samples in the bottom chart were adulterated with varying amounts of cellulose. 


\section{Gingko Leaves Adulterated with Green Tea Leaves}

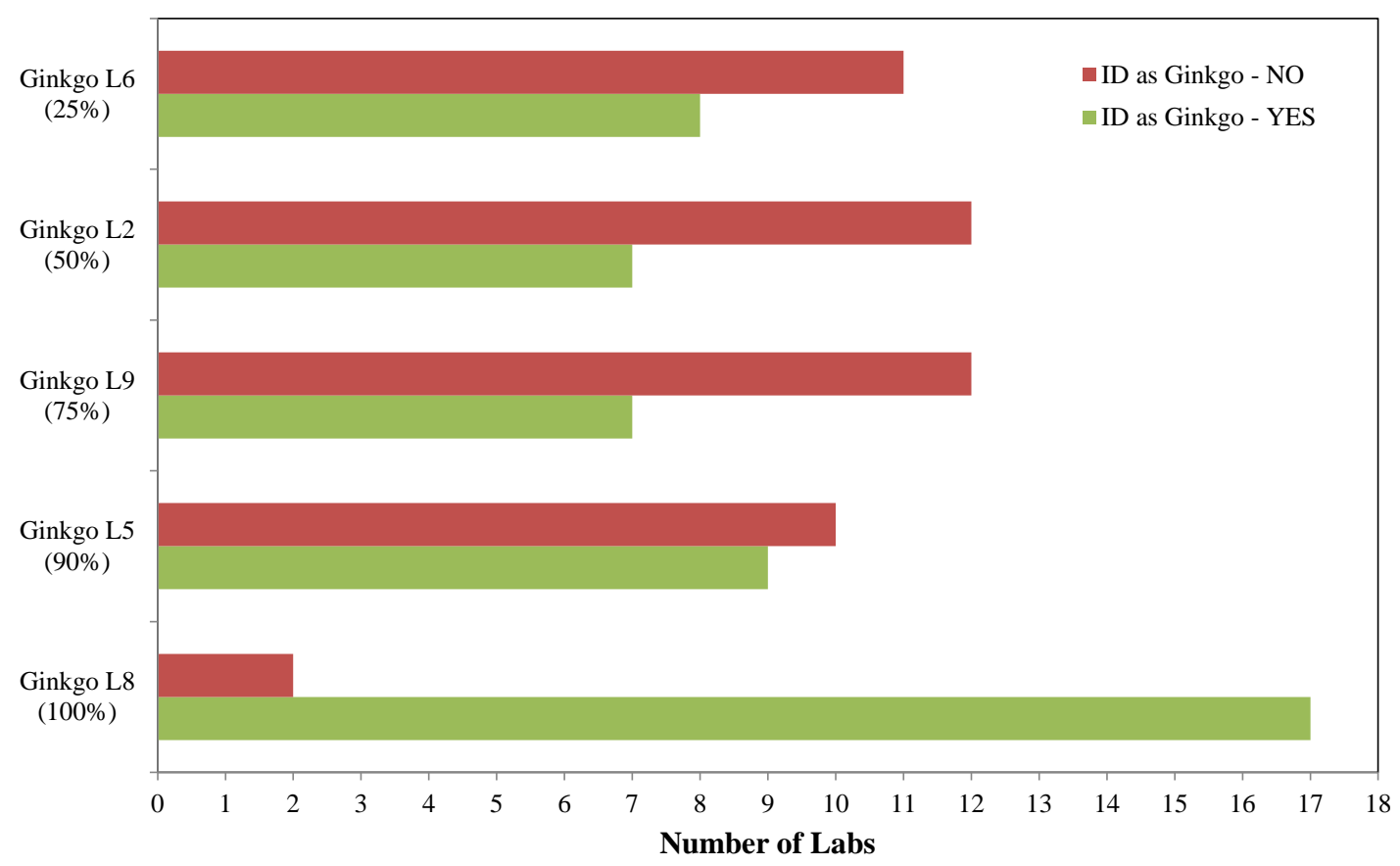

\section{Gingko Leaves Adulterated with Cellulose}

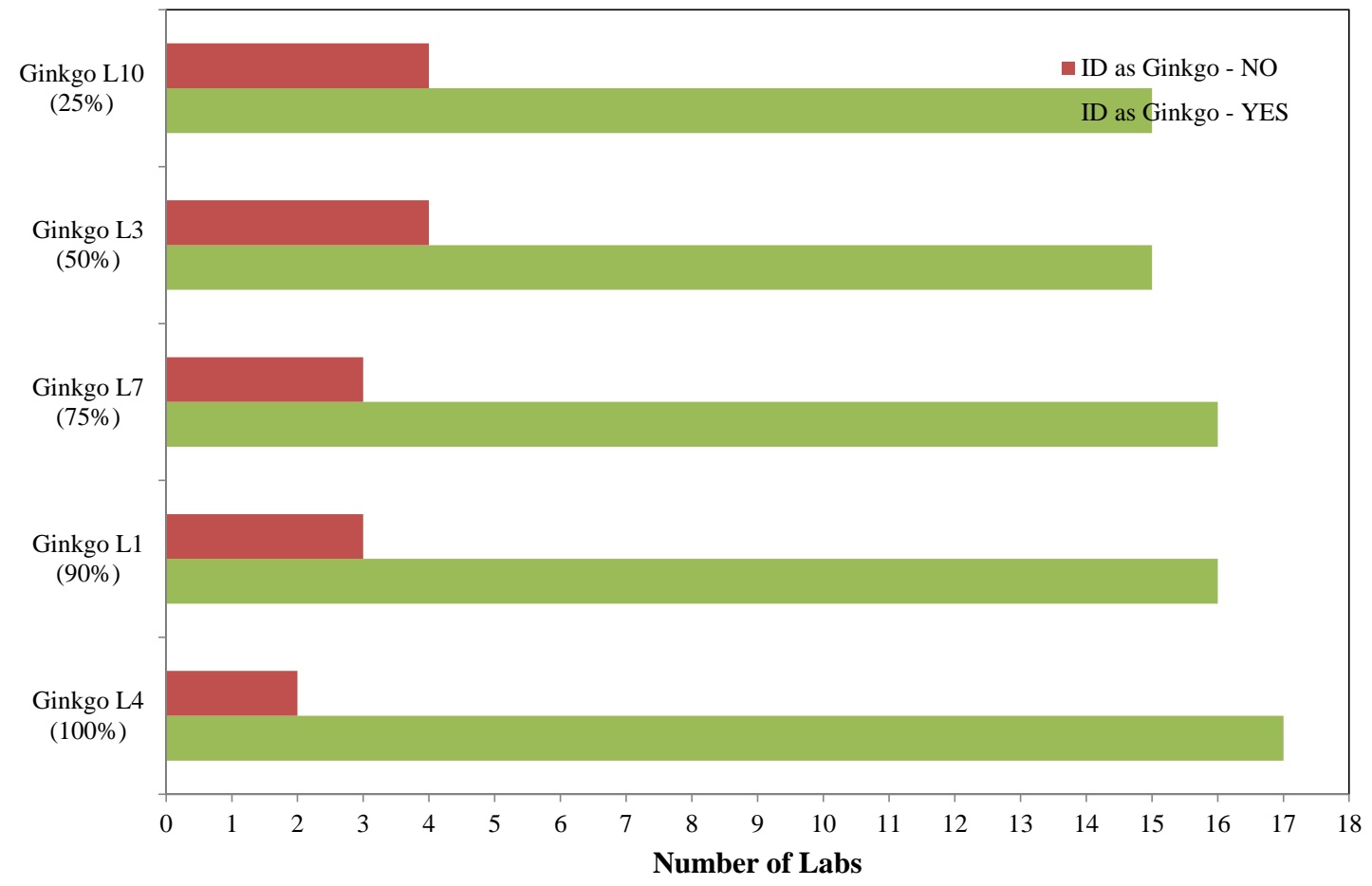

Figure 71. Adulterated Ginkgo biloba leaves. These two charts show the number of laboratories reporting Ginkgo leaves as being adulterated. The samples in the top chart were adulterated with varying amounts of green tea leaves. The samples in the bottom chart were adulterated with varying amounts of cellulose. 